
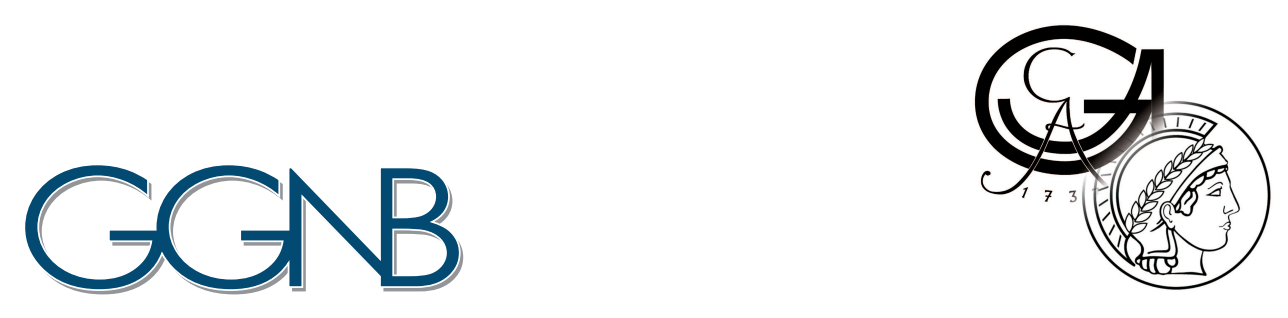

\title{
Structural, biochemical and computational studies on PROPPINs, proteins important in autophagy
}

\author{
Dissertation \\ for the award of the degree \\ "Doctor rerum naturalium" (Dr. rer. nat.) \\ Division of Mathematics and Natural Sciences \\ of the Georg-August-Universität Göttingen
}

submitted by

Andreea Scacioc

from

Constanta, Romania

Göttingen, 2014 


\section{Members of the Thesis Committee:}

Dr. Karin Kühnel ( $1^{\text {st }}$ Reviewer)

Research Group Autophagy, Department of Neurobiology, Max Planck Institute for Biophysical Chemistry

Prof. Dr. Michael Thumm ( $2^{\text {nd }}$ Reviewer)

Department of Biochemistry II, University of Göttingen

Prof. Dr. Blanche Schwappach

Department of Biochemistry I, University of Göttingen

\section{Dr. Martin Kollmar}

Research Group Systems Biology of Motor Proteins, Department of NMR Based Structural Biology, Max Planck Institute for Biophysical Chemistry

\section{Prof. Dr. Helmut Grubmüller}

Theoretical and Computational Biophysics Department, Max Planck Institute for Biophysical Chemistry

\section{Dr. Manfred Konrad}

Research Group Enzyme Biochemistry, Max Planck Institute for Biophysical Chemistry

Date of the oral examination: $17^{\text {th }}$ of December 2014 


\section{Declaration of Authorship}

Hereby, I confirm that I tried to create this work Structural, biochemical and computational studies on PROPPINs, proteins important in autophagy entirely on my own. However, I had to change parts of it according to what my reviewers, Dr. Karin Kühnel and Prof. Dr. Michael Thumm insisted upon without allowing for a discussion. The main changed parts were model Figures 5.14 and 7.4 The change made these models inconsistent with the results in Section 3.2.2 and the model presented in Figure 7.2 in the Discussion part. I dissent from these changes. I complained to both the dean of the GGNB graduate school, Prof. Dr. Reinhard Jahn and GGNB coordination office and my complaints were disregarded since senior researchers know better. Moreover, I was told that if I do not make the changes, my thesis will get rejected and I will not have another chance for recourse. In doing so, I got the Promotionsordnung misquoted by both the GGNB Coordination Office and Dr. Karin Kühnel.

In a similar vein, my supervisor, Dr. Karin Kühnel rewrote parts of the thesis as this is what she understands as suggestions and corrections. I dissent from this attitude as I believe that a PhD thesis should be completely written by its author to show scientific maturity and independence.

In conclusion, I submitted this PhD thesis under the current form, which I disagree with, because I was not given another alternative to obtain the Doctor title I worked for three and a half years.

Göttingen, 16th of December 2015

Andreea Scacioc 
Dedicated to Ángel, Inessa, Karin, Ricarda and Tales because without you, there would have been no PhD thesis 



\section{Abstract}

PROPPINs ( $\beta$-propellers that bind polyphosphoinositides) are conserved eukaryotic proteins that play an important role in autophagy. Yeast contains three PROPPINs: Atg18, Atg21 and Hsv2 (homologous with swollen vacuole phenotype 2), which specifically bind PI3P and PI(3,5) $\mathrm{P}_{2}$. Based on the crystal structure of Kluyveromyces lactis Hsv2, which was determined earlier in our group, I performed docking studies to characterize PI3P and PI $(3,5) \mathrm{P}_{2}$ binding in the two binding sites present in PROPPINs. Based on these docking studies I proposed a model where PROPPINs bind perpendicular to the membrane through their two phosphoinositide binding sites. In addition to the two phosphoinositide binding sites loop 6CD is also required for membrane targeting of PROPPINs. Using coarse-grained and atomistic molecular dynamics simulations I showed that loop 6CD inserts into the lipid bilayer and acts as an anchor for membrane binding of PROPPINs.

I also determined the $1.8 \AA$ resolution crystal structure of Pichia angusta Atg18, which represents the first high resolution PROPPIN structure. As Hsv2 it forms a seven bladed $\beta$-propeller with a non-velcro like propeller closure topology. In order to experimentally validate my proposed PROPPIN-membrane binding model I designed mutants based on the P. angusta Atg18 crystal structure and set-up a fluorescence based assay to measure their distances to the membrane. Initial measurements confirm this model.

Through extensive through isothermal titration calorimetry measurements I quantified PI3P and PI(3,5) $\mathrm{P}_{2}$ binding of P. angusta Atg18, K. lactis Atg21 and S. cerevisiae Hsv2. These PROPPINs bind phosphoinositides with nanomolar and low-micromolar affinities and both Atg18 and Hsv2 bind tighter to $\mathrm{PI}(3,5) \mathrm{P}_{2}$. Analysis of single binding site mutants further showed that the affinities of these mutants are 15- to 30-fold lower compared to the wild-type protein which explains that PROPPINs need two phosphinositide binding sites in order to achieve high affinity binding to membranes. Taken together by combining computational studies, X-ray crystallography and other biophysical methods I gained new insights how PROPPINs interact with phosphoinositides on a molecular level. 


\section{Contents}

\section{Introduction}

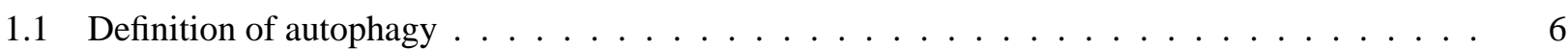

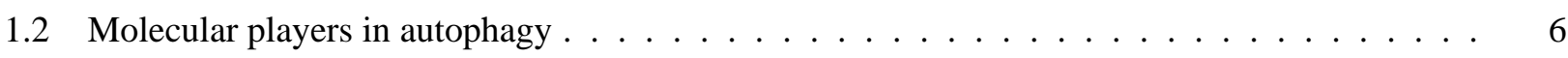

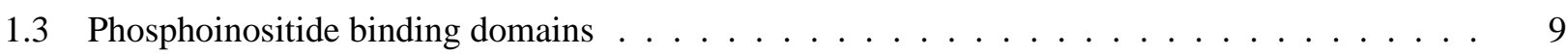

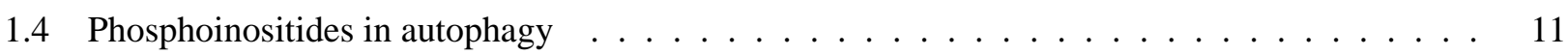

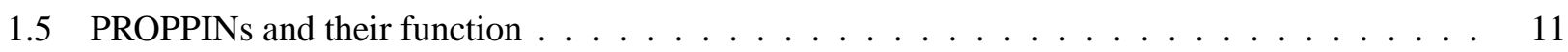

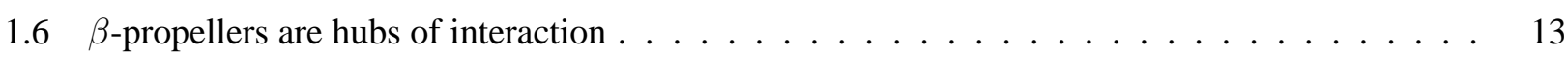

1.7 Interaction partners of PROPPINs $\ldots \ldots \ldots \ldots \ldots \ldots \ldots$

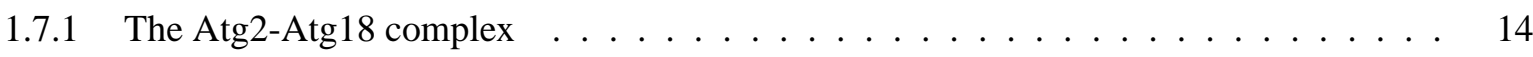

1.7.2 Arabidopsis thaliana Atg18a-WRKY33 complex . . . . . . . . . . . . 16

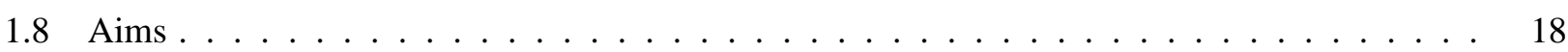

2 Materials and Methods 19

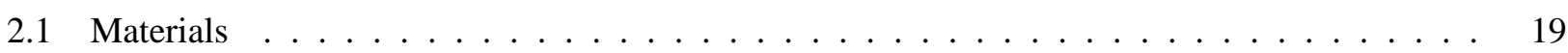

2.1 .1 Chemicals . . . . . . . . . . . . . . . . . . . . . 19

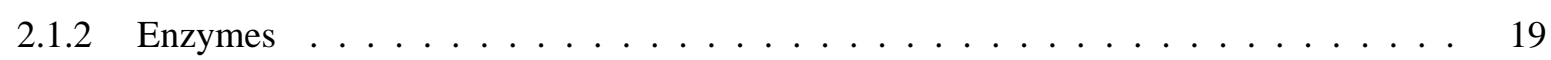

2.1 .3 Lipids . . . . . . . . . . . . . . . . . . . . . . 20

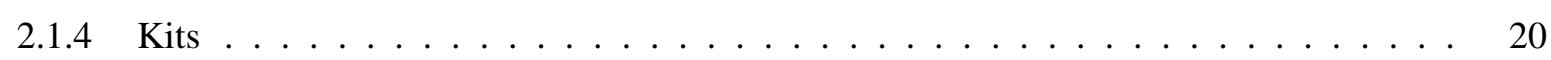

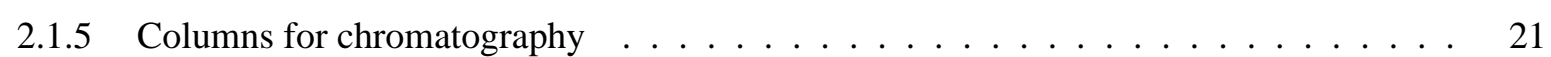

2.1 .6 Antibodies . . . . . . . . . . . . . . . . . . . 21

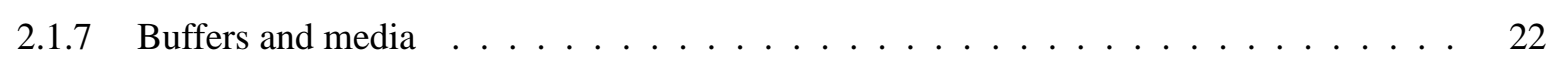

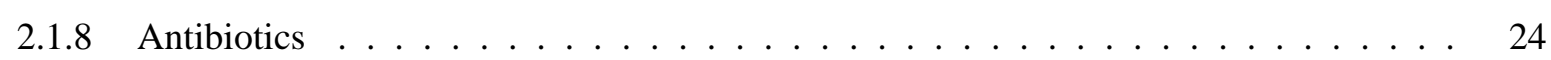

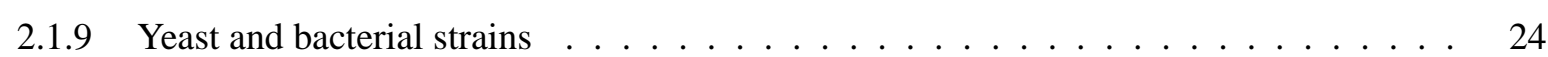

2.1 .10 DNA constructs . . . . . . . . . . . . . . . . . . . 24

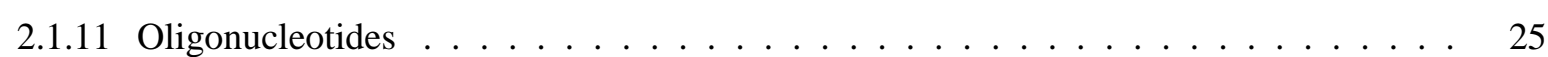

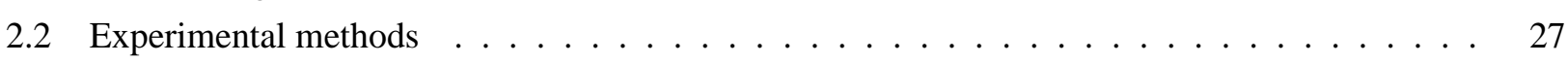

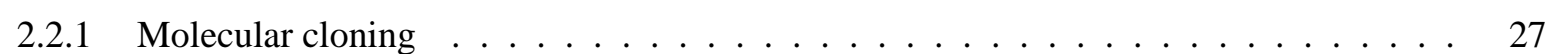

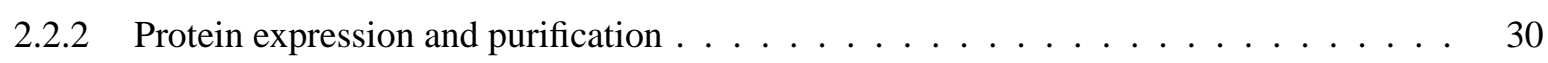

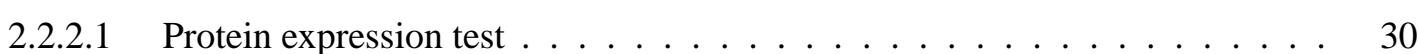

2.2.2.2 Protein expression and purification . . . . . . . . . . . . . . . . . . . 30

2.2 .3 Biochemical methods . . . . . . . . . . . . . . . . . 32

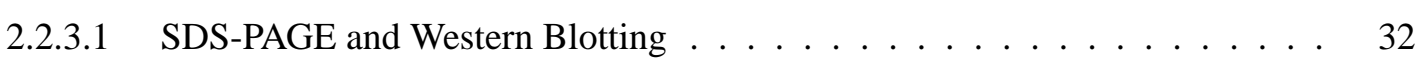

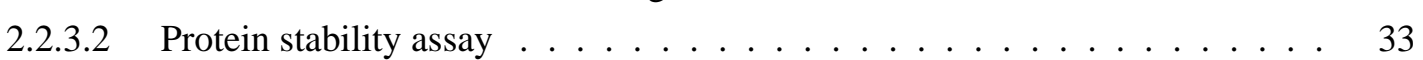


2.2.3.3 Circular dichroism spectroscopy _................. 33

2.2.3.4 Protein-lipid co-flotation assay and liposome preparation . . . . . . . . 33

2.2.3.5 Fluorescence determination of PaAtg18 membrane orientation . . . . . . . 34

2.2.3.6 Isothermal titration calorimetry . . . . . . . . . . . . . . . . . . . . . . . . . .

2.2.4 Crystallization and structure determination $\ldots \ldots \ldots \ldots \ldots \ldots \ldots$

2.2.4.1 Crystallization screen setup . . . . . . . . . . . . . 35

2.2.4.2 Flash cooling of crystals . . . . . . . . . . . . . . 36

2.2.4.3 Computational methods for structure determination . . . . . . . . 36

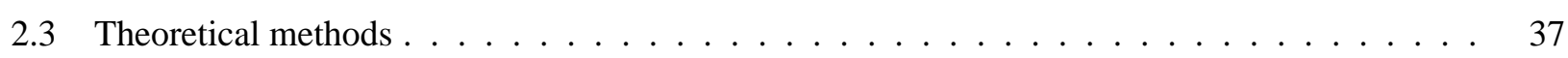

2.3.1 Introduction to molecular dynamics . . . . . . . . . . . . . . . . 37

2.3 .2 Trajectory analysis . . . . . . . . . . . . . . . . . . . . . . . . . . . . . . .

2.3.2.1 Root mean square deviation from a reference structure . . . . . . . . . . . 39

2.3.2.2 Root mean square fluctuations from a reference structure . . . . . . . . . 39

2.3.3 MD simulations for the ligand docking and ligand docking studies with KlHsv2 . . . 39

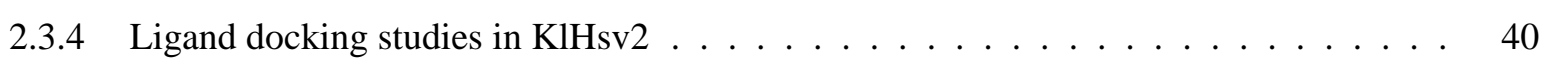

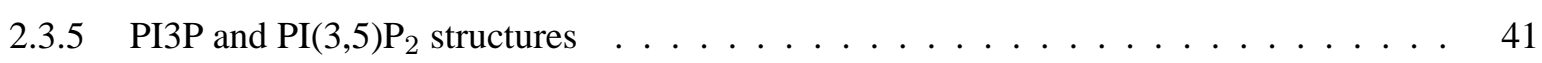

2.3.6 Protein preparation for coarse-grained molecular dynamics simulations of KlHsv2 . . 41

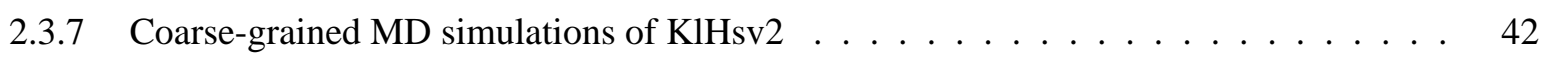

2.3.8 DPPC bilayer formation around loop 6CD of KlHsv2 . . . . . . . . . . . . . 42

2.3.9 Coarse-grained MD simulation of the membrane-KlHsv2 system . . . . . . . . . . 43

2.3.10 Atomistic MD simulations of the KlHsv2-membrane system . . . . . . . . . . . . . . . . . . . . . . . . . . . . .

2.3.11 Homology modeling . . . . . . . . . . . . . . . 43

3 Computational studies of PROPPINs membrane binding 45

3.1 Specific membrane binding of $\mathrm{Hsv} 2 \ldots \ldots \ldots \ldots \ldots \ldots \ldots$

3.1.1 In silico analysis of phosphoinositides binding of KlHsv2 . . . . . . . . . . . 46

3.1.1.1 Computational docking of PI3P and $\mathrm{PI}(3,5) \mathrm{P}_{2}$ in the two binding sites of KlHsv2 . . . . . . . . . . . . . . . . . . 46

3.1.1.2 Dynamics of the binding sites in the bound and unbound form . . . . . . . 49

3.1.2 Phosphoinositides membrane binding of ScHsv2 and K1Atg21 . . . . . . . . . 51

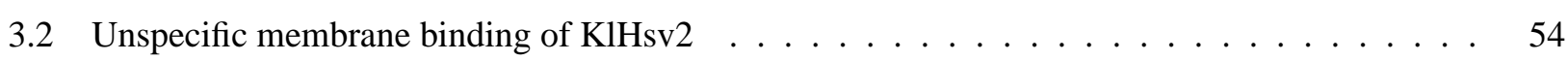

3.2.1 Preliminary model of KlHsv2 membrane binding . . . . . . . . . . . 54

3.2.2 Membrane insertion of loop 6CD based on molecular dynamics simulations . . . . . 54

4 Biochemical characterization of the phosphoinositides binding specificities of PROPPIN family members

4.1 Expression and purification of PROPPINs $\ldots \ldots \ldots \ldots \ldots \ldots$

4.1.1 Expression and purification of $S$. cerevisiae $\operatorname{Atg} 18 \ldots \ldots \ldots \ldots \ldots$

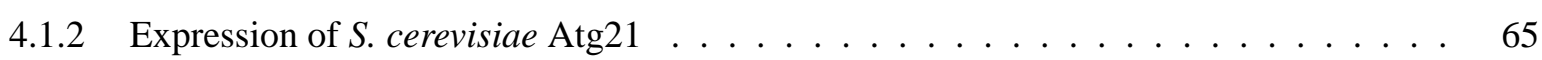

4.1 .3 Expression and purification of $P$. angusta $\operatorname{Atg} 18 \ldots \ldots \ldots \ldots \ldots \ldots$

4.1.4 Expression and purification of K. lactis Atg21 . . . . . . . . . . . . . . . . . . 69 
4.1.5 Expression and purification of S.cerevisiae $\mathrm{Hsv} 2 \ldots \ldots \ldots$. . . . . . . . . . . . . . . . . . . . .

4.1.6 Expression and purification of $C$. thermophilum $\operatorname{Atg} 18 \ldots \ldots \ldots \ldots$. . . . . . . 72

4.2 ITC measurements of PROPPINs with PI3P and PI(3,5) $\mathrm{P}_{2}$ containing liposomes . . . . . . 77

4.3 ITC measurements of wild type versus single binding site mutants of S. cerevisiae Hsv2 with

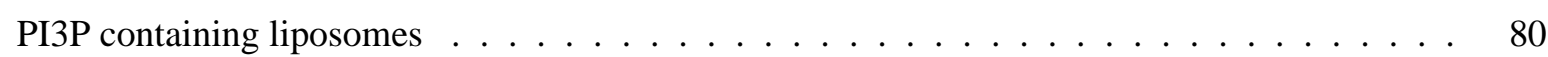

5 Structural characterization of $P$. angusta Atg18 $\quad 82$

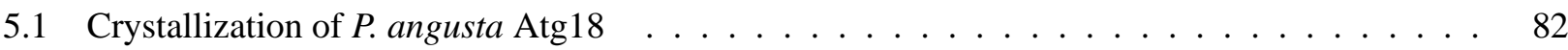

5.1.1 Full length $P$. angusta Atg18 crystallization . . . . . . . . . . . . . . . . 82

5.1.2 In situ proteolysis for crystallization of P. angusta $\operatorname{Atg} 18 \ldots \ldots \ldots \ldots$. . . . . . 83

5.2 Structure determination of $P$. angusta $\operatorname{Atg} 18 \ldots \ldots \ldots \ldots \ldots$

5.2 .1 Data collection and processing . . . . . . . . . . . . . . . . 84

5.2.2 SAD phasing with selenomethionine labeled P. angusta Atg18 crystals . . . . . . . . 88

5.2 .3 Refinement and structure validation . . . . . . . . . . . . . . . 88

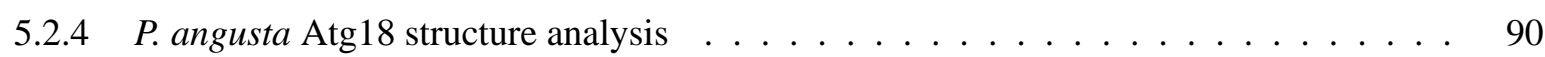

5.3 Analysis of P. angusta Atg18 membrane binding orientation using fluorescence measurements 96

5.3.1 Selection of reporter positions for fluorescence labeling . . . . . . . . . . . . . 96

5.3.2 Membrane binding and folding control of the P. angusta Atg18 mutants . . . . . . . 96

5.3.3 P. angusta $\operatorname{Atg} 18$ membrane binding orientation . . . . . . . . . . . . . . 100

6 Expression and purification of PROPPINs binding partners $\quad 101$

6.1 The $\operatorname{Atg} 2-\operatorname{Atg} 18$ complex $\ldots \ldots \ldots \ldots \ldots \ldots \ldots \ldots \ldots \ldots$

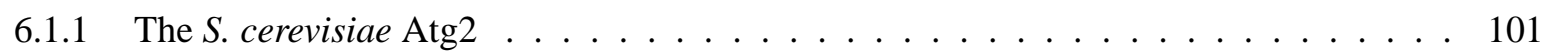

6.1.1.1 S. cerevisiae Atg2 expression optimization in S. cerevisiae . . . . . . . . . . 101

6.1.1.2 S. cerevisiae Atg2 domains expression optimization in E. coli . . . . . . . . 102

6.1.2 C. thermophilum Atg2 domains expression optimization in E. coli . . . . . . . . . . . 104

6.2 The A. thaliana Atg18a-WRKY33 complex . . . . . . . . . . . . . . . . . . . 107

7 Discussion $\quad 112$

7.1 Computational studies of PROPPIN membrane binding . . . . . . . . . . . . . . 112

7.2 Biochemical characterization of phosphoinositides binding specificities of different PROPPIN

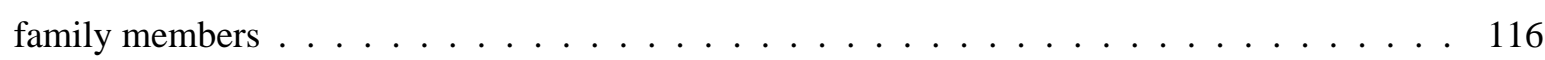

7.3 Structural and biochemical characterization of P. angusta $\operatorname{Atg} 18 \ldots \ldots \ldots \ldots \ldots$

7.4 Expression and purification of PROPPIN binding partners . . . . . . . . . . . . 121

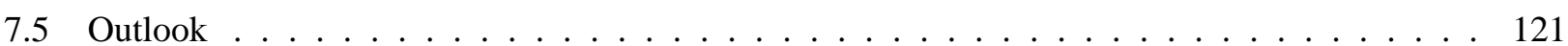

8 Appendix 123

8.1 DNA sequences of synthetic genes . . . . . . . . . . . . . . . . . . . . . 134

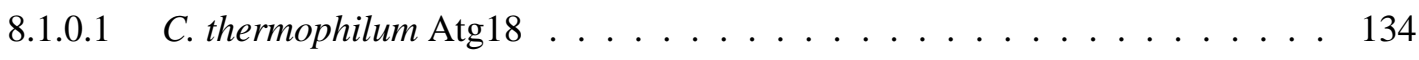

8.1.0.2 C. thermophilum $\operatorname{Atg} 2 \ldots \ldots \ldots \ldots \ldots \ldots$

$8.1 .0 .3 \quad$ A. thaliana $\operatorname{Atg} 18 \mathrm{a} \ldots \ldots \ldots \ldots \ldots \ldots \ldots$

8.1.0.4 A. thaliana WRKY33 . . . . . . . . . . . . . . . . 136 
CONTENTS

References 


\section{List of Figures}

1.1 Schematic overview of autophagy and its subtypes . . . . . . . . . . . 7

1.2 Phosphoinositide binding domains: structure and specificity . . . . . . . . . . 10

1.3 Structural details of KlHsv2, the first PROPPIN paralog structurally characterized . . . . 12

1.4 There are two working hypotheses for the recruitment of Atg2-Atg18 complex to the autophagic membranes ....................... 15

1.5 Importance of Atg18a-WRKY33 complex in the plant resistance to necrotrophic pathogens

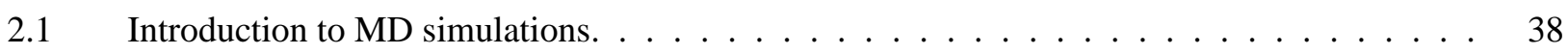

2.2 MD simulation methodology schematic work flow. . . . . . . . . . . . . . . 40

2.3 Ligand docking methodology schematic work flow. . . . . . . . . . . . . . . 41

2.4 Atomic partial charge parametrization of PI3P for GROMOS96 force field. . . . . . . . . 42

2.5 Homology modeling methodology schematic work flow . . . . . . . . . . . . . . 44

$3.1 \quad$ Sulfates bound in the KlHsv2 structure . . . . . . . . . . . . . . . . . . . 45

$3.2 \quad$ W277 release from crystal packing contacts by MD simulation. . . . . . . . . . . 46

$3.3 \quad$ PI3P docking in binding sites 1 and $2 \ldots \ldots \ldots \ldots \ldots \ldots$

$3.4 \quad \mathrm{PI}(3,5) \mathrm{P}_{2}$ docking in binding sites 1 and $2 \ldots \ldots \ldots \ldots \ldots \ldots$

3.5 Dynamics of the KlHsv2 binding sites in the bound and unbound form . . . . . . . . . . 50

3.6 Root-mean-square fluctuations (RMSFs) of the $\mathrm{C} \alpha$ atoms as a function of residue number. . 51

3.7 Multiple sequence alignment of PROPPINs. . . . . . . . . . . . . . . 52

3.8 Essential residues in PI binding for ScHsv2 and KlAtg21 as revealed by homology modeling 53

3.9 Model for PROPPIN membrane recognition. . . . . . . . . . . . . . . . . . . . . . . . . .

3.10 Optimization of the coarse-grained representation for KlHsv2 . . . . . . . . . . . . . . 56

3.11 Time course of a coarse-grained molecular dynamics simulation of DPPC (Dipalmitoylphosphatidylcholine) bilayer formation around loop 6CD of $K$. lactis Hsv2. . . . . . . . . . . 57

3.12 Time course of a $1 \mu$ s coarse-grained molecular dynamics simulation of KIHsv2 in a DPPC (Dipalmitoylphosphatidylcholine) membrane. . . . . . . . . . . . . . 58

3.13 Time course of a $100 \mathrm{~ns}$ atomistic molecular dynamics simulation of KlHsv2 in a DPPC (Dipalmitoylphosphatidylcholine) membrane. . . . . . . . . . . . . 5 58

3.14 Analysis of the molecular dynamics simulations of KlHsv2 in a DPPC (Dipalmitoylphosphatidylcholine membrane. . . . . . . . . . . . . . . . . 59

$4.1 \quad$ Expression optimization for $\mathrm{ScAtg} 18$ in E. coli . . . . . . . . . . . . . 62

$4.2 \quad$ Purification of ScAtg18 from E. coli Rosetta2(DE3)pLysS . . . . . . . . . . . . . 63 
4.3 Stability analysis of $\mathrm{ScAtg} 18$ in several buffers $\ldots \ldots \ldots \ldots$

4.4 Expression optimization for ScAtg21 in E. coli . . . . . . . . . . . . . . . 66

4.5 Buffer optimization for the purification of PaAtg18 and its stability analysis. . . . . . 68

$4.6 \quad$ Purification of PaAtg18 no cysteines, S81C mutant from E. coli BL21(DE3) . . . . . . 70

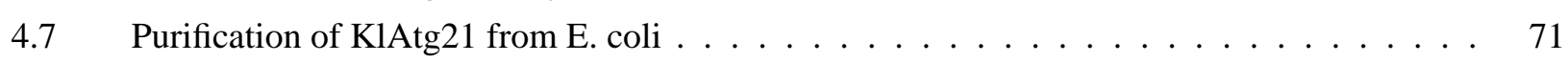

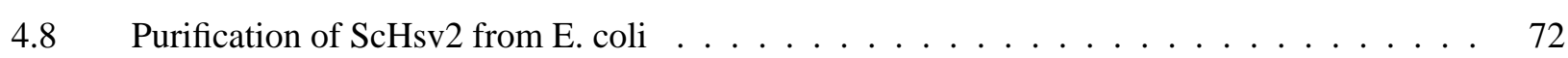

4.9 Stability of wild type ScHsv2 and mutants as measured through CD . . . . . . . . . 73

4.10 Expression tests for CtAtg18 in different expression vectors . . . . . . . . . . . 74

4.11 Expression tests CtAtg18 in pAce1-N-His expression vector in E. coli B121(DE3) . . . . . 76

4.12 ITC measurements of $\mathrm{PaAtg} 18$ with liposomes containing $\mathrm{PI} 3 \mathrm{P}$ or $\mathrm{PI}(3,5) \mathrm{P}_{2} \ldots \ldots$. . . . 78

4.13 ITC measurements of KlAtg21 with liposomes containing $\mathrm{PI} 3 \mathrm{P}$ or $\mathrm{PI}(3,5) \mathrm{P}_{2} \ldots \ldots$

4.14 ITC measurements of $\mathrm{ScHsv} 2$ with liposomes containing $\mathrm{PI} 3 \mathrm{P}$ or $\mathrm{PI}(3,5) \mathrm{P}_{2} \ldots \ldots \ldots$

4.15 ITC measurements of single binding site ScHsv2 mutants with liposomes containing PI3P .

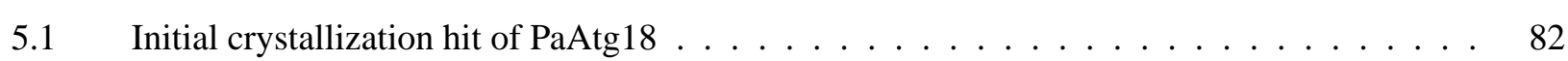

$5.2 \quad$ Full length $\operatorname{PaAtg} 18$ crystal diffracting to $7.7 \ldots \ldots \ldots \ldots \ldots$

5.3 Crystallization strategies tried for $\operatorname{PaAtg} 18 \ldots \ldots \ldots \ldots \ldots$

5.4 Selection of crystallization hits for PaAtg18 in situ proteolysis . . . . . . . . . . 85

5.5 Selection of crystallization hits for selenomethionine labeled PaAtg18 in situ proteolysis . . 86

5.6 Fluorescence spectrum and a scan of Selenomethionine labeled PaAtg18 crystal . . . . . 87

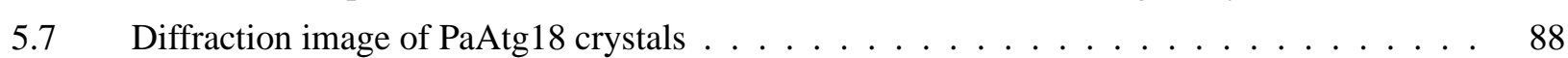

5.8 Experimental $2 \mathrm{mFo}-\mathrm{DFc}$ electron density map contoured at 1.0 sigma with overlaid PaAtg 18

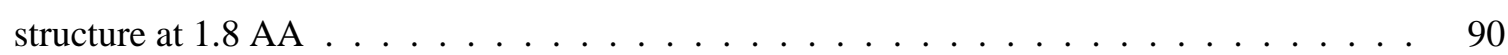

$5.9 \quad$ Refinement statistics for PaAtg18 structure . . . . . . . . . . . . . . . 91

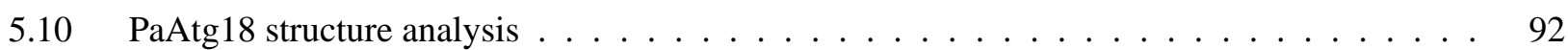

5.11 Crystal bound ligands for the determined PaAtg18 structures. . . . . . . . . . . . 93

5.12 PaAtg18 phosphoinositides binding pockets . . . . . . . . . . . 95

5.13 Multiple sequence alignment of yeast Atg18 homologs . . . . . . . . . . . . . . . 97

5.14 Computational model for PaAtg18 membrane binding . . . . . . . . . . . . . 98

5.15 Binding and folding control of single cysteine PaAtg18 mutants . . . . . . . . . . . 99

5.16 Analysis of PaAtg18 membrane binding orientation through fluorescence . . . . . . . . 100

6.1 Expression optimization for $\mathrm{ScAtg} 2$ in $\mathrm{S}$. cerevisiae $\ldots \ldots \ldots \ldots \ldots$

6.2 Affinity purification of $\mathrm{ScAtg} 2$ from S. cerevisiae . . . . . . . . . . . . . 103

6.3 Expression optimization for ScAtg2 N-terminal fragments in E. coli . . . . . . . . . . 104

6.4 Expression optimization for $\mathrm{CtAtg} 2(1-168)$ in E. coli . . . . . . . . . . . . . 105

6.5 Expression optimization for CtAtg2(1-168)-CtAtg18 complex in E. coli . . . . . . . . . 106

6.6 Expression optimization for CtAtg2 N-terminal fragments in E. coli . . . . . . . . . . 108

6.7 Purification of MBP-CtAtg2(1-279) from E. coli . . . . . . . . . . . . . . 109

6.8 Expression optimization for AtAtg18a in E. coli $\ldots \ldots \ldots \ldots \ldots$

6.9 Expression optimization for AtWRKY33 in E. coli . . . . . . . . . . . . . . . 111 
7.1 Comparison between the conformation of W277 (W288) in the determined structures of KlHsv2 (pdb accession code 4AV9, [1]) and KmHsv2 (pdb accession code 3VUA [2]) . . . 112

7.2 Proposed model for PROPPIN membrane recruitment and binding . . . . . . . . . . 115

7.3 Comparison Atg18 and Hsv2 PROPPIN structures . . . . . . . . . . . . . . 118

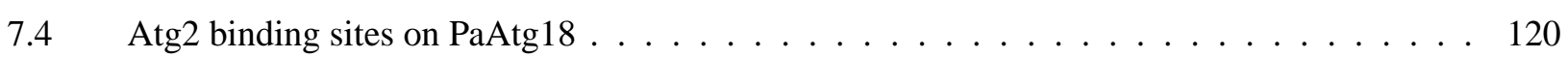

8.1 Electrostatic and hydrophobic contributions to KlHsv2 membrane binding. . . . . . . . 123

8.2 ITC measurements of KlHsv2 with liposomes containing $\mathrm{PI} 3 \mathrm{P}$ or $\mathrm{PI}(3,5) \mathrm{P}_{2} \ldots \ldots \ldots 123$

8.3 Folding controls for PaAtg18 single cysteine mutants . . . . . . . . . . . 133 


\section{List of Tables}

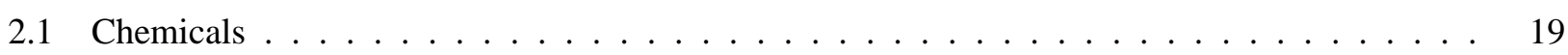

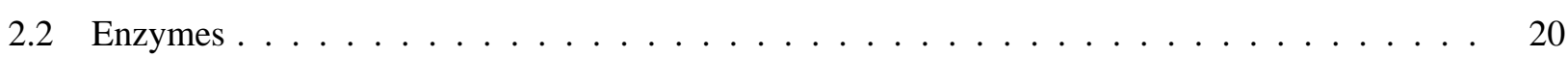

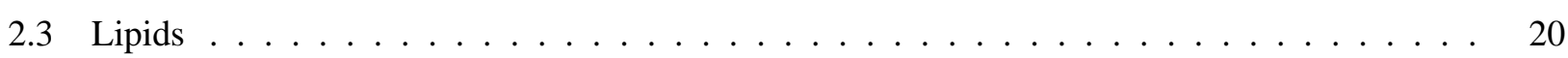

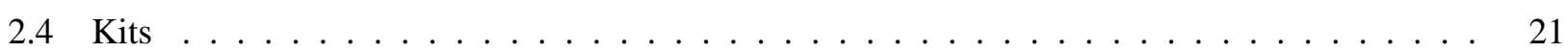

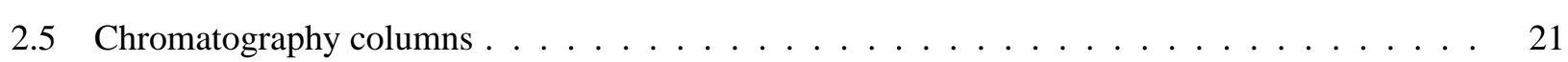

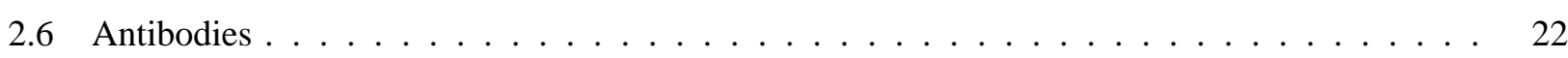

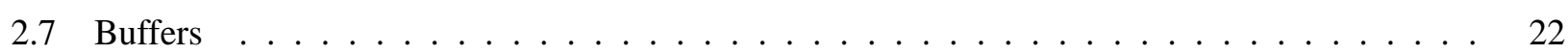

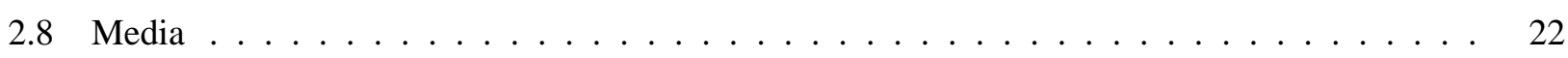

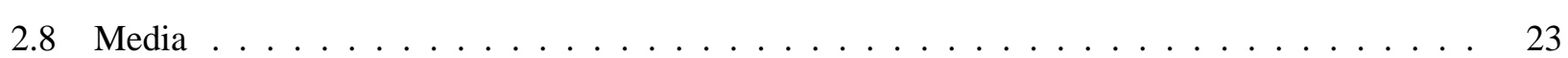

2.9 DNA constructs generated and used in this study $\ldots \ldots \ldots \ldots \ldots \ldots$

2.10 Oligonucleotides ordered especially for this study . . . . . . . . . . . . . . 26

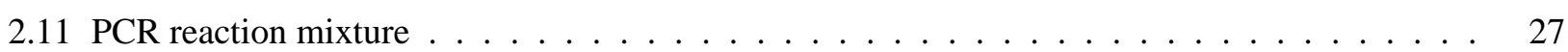

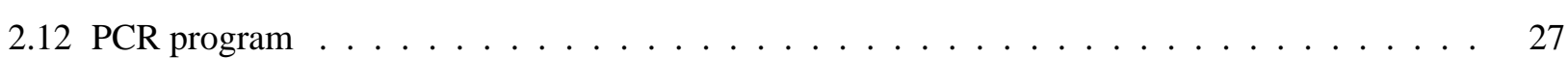

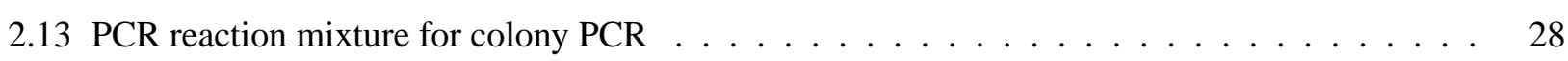

2.14 PCR program for colony $\mathrm{PCR} \ldots \ldots \ldots \ldots \ldots \ldots \ldots$

2.15 PCR program for mutagenesis . . . . . . . . . . . . . . . . . . 29

2.16 Expression conditions for proteins used in this study $\ldots \ldots \ldots \ldots$

2.17 Purification program for HisTrap. $\mathrm{X}^{*}$ - injected volume. . . . . . . . . . . . 31

2.18 Purification program for GSTrap and StrepTrap . . . . . . . . . . . . . . . 31

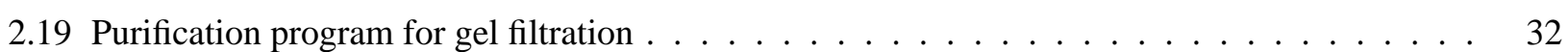

3.1 Summary of all the contacts, salt-bridges or hydrogen bonds, revealed during the docking experiments as shown in Appendix tables 8.1 8.4 In parentheses the count of docking conformations in which these contacts are made is given.

3.2 Comparison of important residues for PROPPINs forming salt bridges with phospoinosites across paralogs of several yeast species.

4.1 Melting temperatures of ScHsv2 wild type and mutants as measured through CD. . . . . . . 72

4.2 Summary of the expression vectors tested for the expression of CtAtg18. The properties of these vectors such as promoter and its inducer, affinity and fusion tags and cloning sites are listed together with the expected protein product size and the cloning sites used for cloning the CtAtg18 gene. MW - molecular weight. 
4.3 Summary of thermodynamic parameters as determined through ITC measurements for PROPPIN PI3P or PI $(3,5) \mathrm{P}_{2}$ binding. $\mathrm{n}$ - number of measurements; $\mathrm{N}$ - binding stoichiometry. Errors reported are standard errors of the mean. . . . . . . . . . . . . . . . 77

4.4 ITC determined binding affinities for wild type $\mathrm{ScHsv} 2$ and its single sites mutants when titrated in PI3P containing SUVs. $\mathrm{n}$ - number of measurements. . . . . . . . . . . . . . . 81

5.1 Sample crystallization conditions for full length PaAtg18 . . . . . . . . . . . . 83

5.2 Selection of successful conditions for in situ proteolysis of PaAtg18. The resulting crystals are shown in Figure $5.4 \ldots \ldots \ldots$. . . . . . . . . . . . . . . . . . . . . . . . . . . . . . . . . . . . . . .

5.3 Data collection parameters for the $1.8 \AA$ selenomethionine PaAtg18 crystal . . . . . . . . 87

5.4 Diffraction data and refinement statistics of the $1.8 \AA$ A selenomethionine PaAtg18 crystal . . . 89

5.5 Fractional coordinates and occupancies for $\mathrm{Se}$ atoms $\ldots \ldots \ldots \ldots$

5.6 Summary of cryoprotectants tested in order to identify the compound in binding site 1 . . . . 91

5.7 Data collection parameters for the $2.3 \AA$ selenomethionine PaAtg18 crystal . . . . . . . . . 93

5.8 Diffraction data and refinement statistics for the phosphate bound $2.3 \AA$ selenomethionine labeled PaAtg18 structure. . . . . . . . . . . . . . . . . . . . . . 94

5.9 Summary of the melting temperatures of the wild type PaAtg18 compared to the single cysteine binding mutants . . . . . . . . . . . . . . . . . . . . . . . . . . . . .

7.1 Summary of reported PROPPIN binding affinities. . . . . . . . . . . . . . . 116

8.1 All contacts, salt-bridges or hydrogen bonds, revealed during the docking experiment for PI3P in binding site 1 of KlHsv2 . . . . . . . . . . . . . . . . . . . 125

8.2 All contacts, salt-bridges or hydrogen bonds, revealed during the docking experiment for $\mathrm{PI}(3,5) \mathrm{P}_{2}$ in binding site 1 of KlHsv2 . . . . . . . . . . . . . . . . . . . . 126

8.3 All contacts, salt-bridges or hydrogen bonds, revealed during the docking experiment for PI3P in binding site 2 of KlHsv2 . . . . . . . . . . . . . . . . . . . . 127

8.4 All contacts, salt-bridges or hydrogen bonds, revealed during the docking experiment for $\mathrm{PI}(3,5) \mathrm{P}_{2}$ in binding site 2 of KlHsv2 . . . . . . . . . . . . . . . . . . . . . 128

8.5 All 96-well screening conditions that led to crystals of PaAtg18. . . . . . . . . . . . 129

8.6 All 96-well screening conditions that led to crystals of PaAtg18. Continuation from Table $8.8 \quad 130$

8.7 All 96-well screening conditions that led to crystals of PaAtg18. Continuation from Table $8.8 \quad 131$

8.8 All 96-well screening conditions that led to crystals of PaAtg18. Continuation from Table $8.8 \quad 132$ 


\title{
List of Abbreviations
}

\author{
ANTH $\ldots . \ldots$. AP180 N-terminal homology \\ Atg...$\ldots \ldots$ Autophagy-related proteins \\ BARA $\ldots . . \ldots \beta-\alpha$ repeated autophagy-specific \\ $\mathrm{C} 2 \ldots \ldots \ldots$ Conserved region-2 of protein kinase $\mathrm{C}$ \\ CD .......... Circular dichroism \\ CG-MD ...... Coarse-grained molecular dynamics \\ CVT pathway . Cytoplasm to vacuole targeting pathway \\ $\mathrm{dH}_{2} \mathrm{O} \ldots \ldots$. deionized water \\ DPPC ....... Dipalmitoyl phosphatidyl choline \\ ENTH ....... Epsin N-terminal homology \\ ER .......... Endoplasmic reticulum \\ FREM ........ Ezrin, radixin, moesin \\ FYVE ........ Fab1, YOTB, Vac1 and EEA1 \\ GABARAP ... $\mathrm{GABA}_{A}$ receptor-associated protein \\ GABARAPL1 . GABARAP-like protein1 \\ GABARAPL2 . GABARAP-like protein2 \\ GOLPH3 $\ldots$. Golgi phosphoprotein 3 \\ IPTG $\ldots \ldots \ldots$ Isopropyl $\beta$-D-1-thiogalactopyranoside \\ ITC $\ldots \ldots \ldots$ Isothermal titration calorimetry \\ $\mathrm{kb}$........... Kilo base \\ LUV ........ Large unilamellar vesicles \\ MAP1LC3A .. Microtubule-associated protein-1 light chain 3A \\ MD simulation Molecular dynamics simulation \\ PAS ......... Preautophagosomal structure \\ PCR ......... Polymerase chain reaction \\ $\mathrm{pdb} \ldots \ldots \ldots$ protein data base \\ PDZ ......... Postsynaptic density 95, disk large, zonula occludens \\ PH $\ldots \ldots \ldots \ldots$ Plekstrin homology \\ PI .......... Phosphoinositide \\ $\mathrm{PI}(3,4) \mathrm{P}_{2} \ldots \ldots$ Phosphatydylinosotol-(3,4) biphosphate \\ $\mathrm{PI}(3,5) \mathrm{P}_{2} \ldots \ldots$ Phosphatydylinosotol-(3,5) biphosphate \\ $\mathrm{PI}(4,5) \mathrm{P}_{2} \ldots \ldots$ Phosphatydylinosotol-(4,5) biphosphate
}


PI3K ........ Phosphatidyl 3-kinase

PI3P ........ Phosphatidyl inositol-3 phosphate

PI4P ....... Phosphatidyl inositol-4 phosphate

PI5P ....... Phosphatidyl inositol-5 phosphate

$\mathrm{PIP}(3,4,5) \mathrm{P}_{3} \ldots$ Phosphatidylinositol- $(3,4,5)$ triphosphate

PROPPIN ..... $\beta$-propellers that bind phosphoinositides

PTB ........ Phosphotyrosine binding

PX ......... Phox homology

RMSD ....... Root mean square deviation

RMSF ....... Root mean square fluctuation

RNA ........ Ribonucleic acid

SAD ........ single anomalous diffraction

SDS-PAGE ... Sodium dodecyl sulfate polyacrylamide gel electrophoresis

sec .......... Seconds

SNARE ...... Soluble NSF Attachment Protein Receptor

SUV ....... Small unilamellar vesicles

Tm .......... Melting temperature

TOR ........ Target of rapamycin

TORC1 ...... Tor complex 1

WIPI ....... WD-40 repeat containing protein that interacts with PIs 




\section{Acknowledgments}

I am grateful to my advisor Dr. Karin Kühnel for giving me the opportunity to do the research in her lab and for continuous advice, both scientific and career-wise. She always was available for one-to-one training and kept an open door for questions and discussions.

One very important person during my PhD thesis was Prof. Dr. Reinhard Jahn. He served as a role model, mentor and supporter. I am grateful to him for offering the generous financial support during my thesis and for his comments and encouragement after each departmental seminar and journal club.

Next, I would like to express my gratitude to my thesis committee formed by Prof. Dr. Blanche Schwappach, Dr. Martin Kollmar and Prof. Dr. Michael Thumm. I was surprised and then especially grateful that my thesis committee always read my reports and I could see that they were well prepared for my presentation. Moreover, they always asked me further knowledge questions and ensured that the track is kept such that I finish on time. The questions showed me where my weak points are and helped me become a better wholesome scientist. Their suggestions were precious since they came from different perspectives and background. In addition, their constant positive evaluation of my results gave me confidence that I am on the right track.

A special thanks goes to my close collaborators in Prof. Thumm's lab, especially to Dr. Roswitha Krick. In the same vein, I am grateful for the financial support my project got from DFG through SFB860. Beside the financial support, the SFB collaboration brought interesting scientific insight into similar research through its retreats. This is how I got to know my close collaborator Sona Pirkuliyeva who, being a fast learner, has surpassed my insights into my liposome based methodology to the point that her questions challenged me to further develop my knowledge.

I would like to also mention my co-authors on the papers I had during my thesis because without them my publications list would be significantly shorter: Dr. Ricarda Busse, Dr. Roswitha Krick, Prof. Dr. Michael Thumm, Prof. Dr. Andreas Janshoff, Dr. Milena Stephan, and Dr. Matias Hernandez.

In terms of facilities and machines used, I would like to thank Dr. Vladimir Pena for leading the crystallography facility and organizing our data collection trips. Moreover, I am grateful to him for the access to the thermocycler used for the Thermofluor experiments. When speaking about the crystallography facility, two people come to my mind: Jürgen Wawrzinek and Ulrich (Uli) Steuerwald. Without Jürgen we would not have anything organized nor any screens ready to use. Beside that, his smile and good words brighten then solitude of the crystallography facility and your day. Uli is the person who complements Jürgen and adds to the needs of the facility. Without his extensive knowledge in crystallography, I would have never been able to reproduce my hit from the 96 wells plate and upscale my crystals. Similarly, the scientific discussions with Almudena Ponce-Salvatierra and Dr. Jana Schmitzova helped me further develop my knowledge. When it comes to crystallography, the experience of Dr. Inessa De and Dr. Tales Rocha de Moura was essential in too many ways 
to describe here from company and support during the long SLS sessions to support and encouragement while fishing my crystals. Without Tales sitting next to me and telling me that I can do it, I would never believe in myself. Besides, when I was stuck into my experiments, Inessa and Tales went so far as ensuring that I eat properly, even though this meant to go vegan grocery shopping. When it comes to crystallography and protein expression and purification, I have to mention Karine dos Santos to who I am looking up to as a rightful role model.

Furthermore, I would like to mention the help of Monika Raabe from the research group of Prof. Dr. Henning Urlaub for the assistance with the mass spectrometry analysis. Similarly, I am grateful to the support staff at the SLS of the Paul Scherrer Institute during the data collection sessions.

In terms of molecular dynamics simulations, I am thankful for the discussions to Prof. Dr. Marcus Müller and many of the Grubmüller lab, especially David Köpfer, Hadas Leonov, Carsten Kutzner, Sarah Rauscher, Reinhard Klement and Christian Kappel. Christian was the one that helped me get started with my MD simulations on the GWDG clusters. Speaking of which, I would like the thank GWDG for access to the Nehalemcluster and all their timely support. A special thanks go to Dr. Christian Boehme. When it comes to computational facilities, I would like to thank Heiko Niemeier for all the help he offered for the crystallization software and Linux updates.

Speaking of MD simulations, I think the person whom everyone who has ever had a question or was in need of scripts has to thank is Dr. Justin Lemkul. He was an inspiration for making science open and help people, even if they are in far away countries and the only means of communication is the internet. The same goes to all my co-moderators on Bioforum. Similarly, I am grateful to the people developing Gromacs, the Martini force field, Sugar-Pie server and all the other free servers available that were used in this thesis.

Two close collaborators deserve special mention (and an explanation why this thesis is dedicated to them): Dr. Ricarda Busse and Dr. Ángel Lara Pérez. Ricarda was the one who put the bases for my project for me to have a successful start with publications from my first year of $\mathrm{PhD}$. She is responsible for me having a fall-back project that actually became my $\mathrm{PhD}$ thesis when the Atg2 project proved to be more challenging than we believed in the beginning. Ricarda was the person who first showed me around the department when I joined and helped integrating me in the social life of the department and GGNB. Besides a good research team mate, Ricarda enter my heart as a close friend forever. If Ricarda helped me start, Ángel was the one who helped me finish. Our first scientific collaboration started when he has helped me with the ITC measurements. Then he came up with the ideas for the fluorescence experiments to characterize the PROPPINs. He made it a priority to help me finalize on time, and I appreciate this since this type of altruistic collegiality is rarely found in the world of science. Ángel is an exceptional scientist, as well as he does science how I feel that it is supposed to be done: slow, careful, helpful and collaborative.

Furthermore, I would like to thank my Master students: Oleksandr (Sasha) Yagensky and Sebastian (Sebi) David. Sasha was an exceptional student that everyone would be lucky to have. In less than two months he cloned, expressed and purified proteins and set crystallization trials. It was pure bad luck that all this effort did not lead to a structure. My dear Sasha, science is unpredictable and it does not reflect your actual potential. Sebi was amazing in cloning and testing all the Atg2 constructs from both S. cerevisiae and C. thermophilum. This was an enormous amount of work for such a short time. Both Sasha and Sebi raised the expectation bar high for any other student I will ever have. 
I want to thank my colleagues and friends Caroline (Caro) Behrens and Janina Metje. They were both helpful with discussions about science and beyond. They were also my shoulder to cry, even if sometimes I was like a broken record on certain topics.

One of the best things about my thesis was the fact that it happened in the Jahn department. Even if Göttingen was mostly rainy, it was sunny here because people are friendly and relaxed, while doing great science. The people that all of us have to thank are Dr. Gottfried (Frieder) Mieskes, Dr. Hans Dieter Schmitt, Ursel Ries and Heike Löffler. Frieder was for most of my $\mathrm{PhD}$ the person who anonymously was making the department function. This task was forwarded to Dieter who is amazing in making the department run and taking care of his research group, while being the Ombudsperson of the institute. He is amazing and my only regret it not telling him quite every day how much I appreciate what he does for us. Heike has probably those tasks that everyone takes for granted and are observed only when she is on vacation or sick. Like she always says: 'If you get a Nobel prize, just say thanks then'. Well, we do not know about that Nobel prize but what I know is that her smile and good words made most of my days. Ursel was the person who first did the phosphate determination for my experiments and then taught me the method. She is one of the most wonderful technicians I had the honor to work with. I also was honoured to work alongside people like Dr. Partho Halder, Dr. Janina Boyken, Dr. Saskia Schröter and Dr. Beyenech Binotti. Partho was my shoulder to cry in many occasions. Interestingly, he never understood how special he is for me. Not only for listening to me but also for thinking about me when he found and interesting scientific review to share or interesting material about my activism. Janina was the person I looked up mostly. She is a thought role model to follow. Her dedication and scientific rigor she showed towards her experiments are a true inspiration. Besides, she is one of those great scientists that show empathy and kindness, which is hard to get but makes you fill amazing when you win it. Beyenech seemed a natural friend to come to the overly career-oriented me because she worked on autophagy too. However, she shortly went far beyond it with her understanding, sensitivity and kindness. Saskia entered my friendship zone without expecting it. She is a though lady but also kind and without her we would not have any of the graduation hats or many of those things you need to show more than collegiality towards the lab mates. She is intelligent, creative and an inspiration for doing stuff outside the lab. But all my lab mates are a continuous inspiration: Dr. Julia Preobraschenski, Halenur Yavuz, Agata Witkowska, Dr. Pradip Kumar Tarafdar, Momchil Ninov, Sabrina Beckmann, Shrutee Jakhanwal, Zohreh Farsi, Mahdokht Kohansalnodehi, Dr. Dominika Czernik, Dragomir Milovanovic, Dr. John Chua, Dr. Matias Hernandez, Dr. Emilio Iraheta, Dr. Esra Demircioğlu, Dr. Wensi Vennekate, Dr. Geert van den Bogaar, Dr. Seiichi Koike, Dr. Yongsoo Park and Tahere Kalantary Dehaghi. Moreover, I would like to mention Brigitte Barg-Kues, Dagmar Diezmann, Wolfgang Berning-Koch, Sigrid Schmidt and Peter Mienkus. Last but not least Elisa Reckmann-Heinrich was great in always being helpful with all of us for all the administrative issues. It took a maternal leave to show us that we really cannot live without her. Literally: we were about to kill each other over organizing the journal clubs. :)

During my PhD I was a part of some communities that furthered my skills, such as GGNB, PhD/Postdoc community at MPI-bpc and Max Planck Society PhDnet. GGNB provided method courses to develop further my lab skills beyond the methods used in my thesis, while also offering a social environment appropriate to scientific discussions and social interaction. PhD/Postdoc community was the place where I met people such as Ulrike, JP, Timo and many others. PhDnet was the closest community to my heart. I was involved in it for 
two years. This made me a better, more balanced person, thanks to Felix, Rika, Natascha, Jan, Prateek, Ana, Clemens, Julian, Zainab and Jeff.

My dear co-mentees made me a better woman scientist. So thank you Natalia, Dominika and Tamara. I will always keep you in my heart and no matter how high or low I will reach in my career, I will know that I will have you by my side. Speaking of mentoring, one very important person that I express my gratitude towards is our mentor, Prof. Dr. Sharmishtha Dattagupta.

Last but not least, my friends from outside the lab were essential to my sanity: Sven, Birgit, Albena, Michi, Andreas, Cornelius, Anita, Ingrid, Miro, and everyone who kept me happy in the last years. With special emphasis on keeping my sanity in the last months and being personally responsible for this thesis being handed in on time in a better form are Karin, Tales, Inessa, Albena, Sven and Saskia. The most important friends and family, beside Tales and Inessa, are Laura, Irina and Teo! 


\section{Introduction}

\subsection{Definition of autophagy}

Autophagy was originally discovered as the cellular process of a cell breaking down its own components under stress conditions such as starvation. It was observed in the 50s by Christian de Duve in electron microscopy pictures. At the time, De Duve was studying the lysosome, for which he got the Nobel prize in 1974. He coined the term 'autophagy', using the Greek words for 'self-eating'.

Since its first physiological description, autophagy was shown to be involved in intracellular clearance of damaged organelles, differentiation, development, programmed cell death, antigen presentation, and elimination of invading pathogens (reviewed in Ref. [3]). Dysfunction in autophagy pathway leads to diseases such as cancer (reviewed in Ref. [4]) and neurodegeneration (reviewed in Ref. [5]).

There are three types of autophagy known: micro- and macroautophagy as well as chaperone-mediated autophagy [6], [3] (schematically represented in Figure 1.1). In macroautophagy, isolation membranes appear in the cytoplasm and enlarge to enclose a portion of the cytoplasm, leading to the formation of a double membrane structure known as an autophagosome. The autophagosome fuses with the lysosome (or vacuole in yeast), where the inner membrane and its components are degraded by enzymes. In the case of microautophagy, the lysosomal membrane (or vacuolar membrane in yeast) invaginates creating a sac containing cytosolic components. This sac encloses and scission occurs such that the vesicle containing the cytosolic components reaches the degradation enzymes in the lysosome (vacuole in yeast). Finally, for chaperone-mediated autophagy [7], targeted cytosolic components are selectively translocated into the lysosome using specific chaperones located on both sides of the lysosomal membrane.

Both macroautophagy and microautophagy can be non-selective and selective. In the case of selective autophagy, special receptors are involved for each of the possible selective autophagy types [6]: mitophagy, xenophagy, piecemeal autophagy, ribophagy, pexophagy etc.

\subsection{Molecular players in autophagy}

The initial microscopy images taken by de Duve gave only hints about the process; for the next 30 years, the understanding of autophagy was slow and based alone on physiological mammalian data. However, in 1992, Yoshinori Ohsumi has shown that the yeast autophagy happens similarly to the process in higher eukaryotes [8]. Considering the fact that yeast is easy to genetically manipulate, this finding allowed the genetic screens that led to the identification of the first molecular components involved in autophagy in the laboratories of 


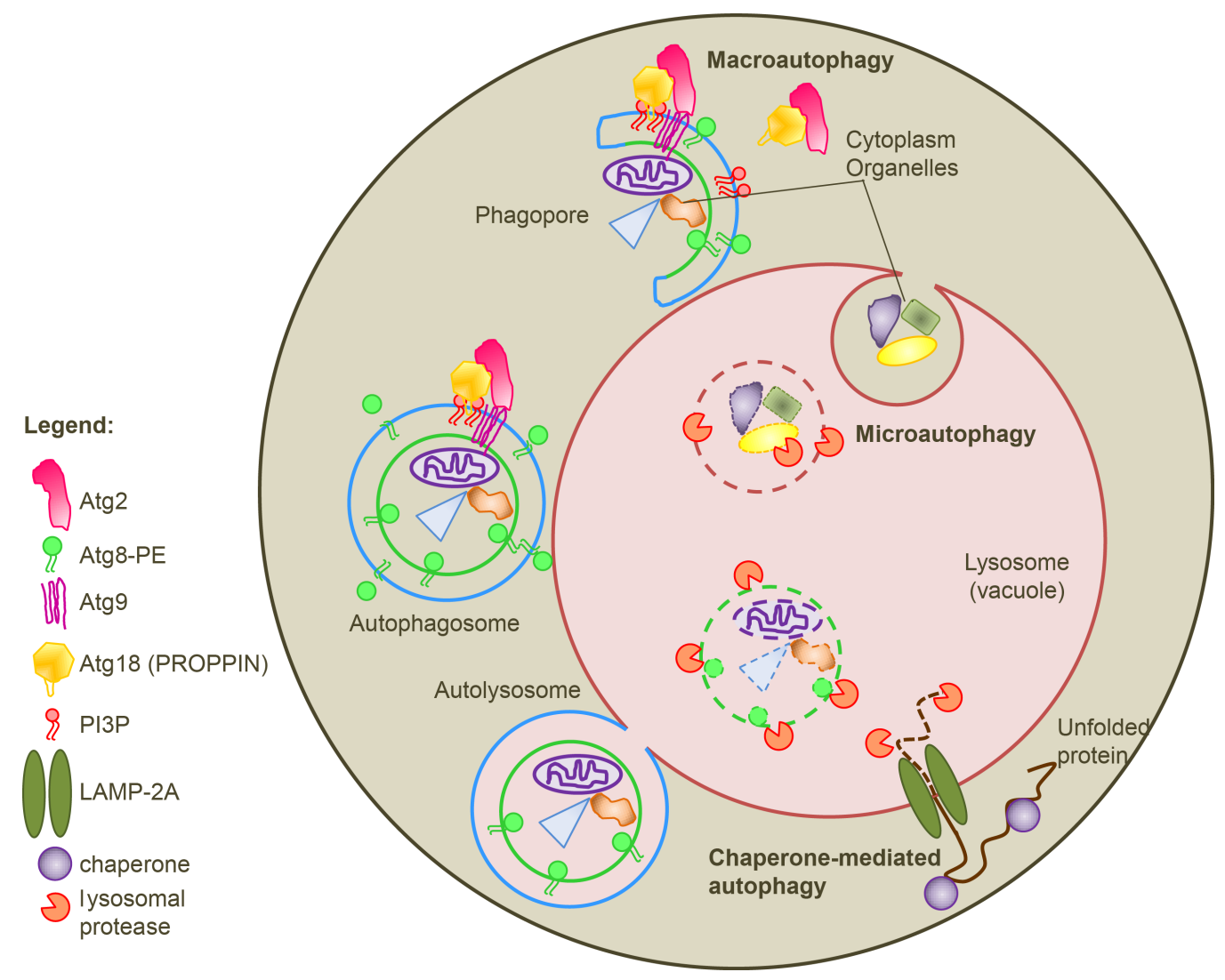

Figure 1.1: Schematic overview of autophagy and its subtypes.

There are three types of autophagy known: micro- and macroautophagy as well as chaperone-mediated autophagy. In macroautophagy, isolation membranes appear in the cytoplasm and enlarge to enclose a portion of the cytoplasm, leading to the formation of a double membrane structure known as an autophagosome. The autophagosome fuses with the lysosome (or vacuole in yeast), where the inner membrane and its components are degraded by enzymes. In the case of microautophagy, the lysosomal membrane (or vacuolar membrane in yeast) invaginates creating a sac containing cytosolic components. This sac encloses and scission occurs such that the vesicle containing the cytosolic components reaches the degradation enzymes in the lysosome (vacuole in yeast). For chaperonemediated autophagy, targeted cytosolic components are selectively translocated into the lysosome using special chaperones located on both sides of the lysosomal membrane.

Klionsky [9], Ohsumi [10] and Thumm [11], as well as in other groups [12, 13, 14, 15]. Initial identification and characterization of autophagy proteins was done in either Saccharomyces cerevisiae or Pichia pastoris.

The steps involved in autophagy are mediated by a number of unique proteins called Atg (autophagyrelated proteins). There are 37 known Atg proteins [6, 16], among which eighteen Atg proteins are essential for the autophagosome formation step [17, 18]. The eighteen genes encoding for these proteins are mostly conserved among higher eukaryotes such as mammals and plants, suggesting that the molecular mechanism of autophagosome formation is also conserved. These Atg proteins are categorized into five functional groups [18]:

- Atg1 protein kinase and its regulators (Atg13, Atg17, Atg29, and Atg31)

- the autophagy-specific phosphatidylinositol 3-kinase complex (Vps30/Atg6, Atg14, Vps15, and Vps34)

- integral membrane protein Atg9 and the Atg2-Atg18 complex

- the Atg8 conjugation system (Atg5, Atg4, Atg7, and Atg8) 
- the Atg12 conjugation system (Atg5, Atg7, Atg10, Atg12, and Atg16)

In the last 20 years since the isolation of the first yeast autophagy-deficient mutants, all 37 Atg proteins were characterized by cell biological and biochemical methods. However, since 'If you want to understand function, study structure' - as Francis Crick said - the last years have seen an explosion in the structural knowledge of Atg proteins, as reviewed in Ref. [19, 20].

In order to better understand the details of the molecular mechanism behind autophagosomal formation, one has to look deeper behind the molecular players involved in each of the above mentioned complexes.

Autophagosome formation is triggered under starvation conditions which inactivates TORC1 (Tor complex1). In nutrient rich conditions, TORC1 hyperphosphorylates Atg13. Dephosphorylated Atg13 associates with Ser/Thr kinase Atg1 and activates it. The ternary complex formed by Atg17, Atg28 and Atg31 binds to Atg1Atg13 complex [21]. This complex activates downstream steps and the cycling of Atg9 between the source for membranes and the PAS (pre-autophagosomal structure) [22]. The basic knowledge about the function of Atg1 kinase and its regulators was obtained through cell biological methods. However, structural biology has recently offered deeper insights. The structure of the At17-Atg28-Atg31 ternary complex has shown that this complex dimerizes in order to fulfill its role [23, 24, 25]. It is hypothesized that this dimerization ensures the vesicle tethering that contributes to the PAS growing into an autophagosome. Next, the structural details of Atg1 interaction with Atg13 and Atg17 have shown that the dephosphorylation of Atg13 leads to its interaction to both Atg1 and Atg17 [26]. Moreover, these structural studies offered the basis for the deeper understanding of the Atg1-Atg13-Atg17-Atg29-Atg31 complex as a dimer of pentamers [27].

The Atg1 and its regulators activate phosphatidylinositol 3-kinase complex. In S. cerevisiae, Vps34 is the only phosphatidylinositol 3-kinase. Vps34 is a member of two distinct complexes that have distinct functions in autophagy (complex I) and in the vacuolar protein sorting (complex II). Complex I is composed of Vps34, Vps15, Vps30/Atg6 and Atg14, whereas Vps38 replaces Atg14 in complex II. Atg14 or Vps38 have an important role in the correct localization of the respective complex where it is needed for the production of phosphatidyl-3 phosphate (PI3P). The structural study of this complex was initiated both in the mammalian Beclin1 (Vps30/Atg6 homolog) [28] and yeast Vps30/Atg6 [29]. The determined structures revealed a novel domain, BARA ( $\beta$ - $\alpha$ repeated autophagy-specific), which is used in PAS recognition. Moreover, the structural study of Beclin1 has revealed information about its interaction with Atg14L and UVRAG (Vps38 human homolog).

Once the PI3P is produced at the PAS, the Atg2-Atg18 is recruited there. Atg18 can bind to both PI3P and $\mathrm{PI}(3,5) \mathrm{P}_{2}$ (phosphatidylinositol-3,5 biphosphate), depending whether it is performing its function at the PAS or at the vacuolar membrane. It is still not understood what the exact function of this complex is, but it is known to be essential for the autophagosome formation. Atg2 is known to interact with Atg9. Atg9 cycles between the PAS and unknown cytosolic membranes. This complex is the least structurally studied out of all autophagic core complexes. Structural information about Atg18 function can be implied from the structure of its paralog, Hsv2 and complementary mutagenesis studies [1, 30, 2].

The best studied complexes in autophagy are by far the ubuiquitin conjugation complexes involving Atg8 and Atg12 Atg5. For each conjugation complex, there is a ubiquitin-like protein: Atg8 and Atg12 [31]. They are conjugated to phosphatidyl ethanolamine and and Atg5, respectively with the help of the Atg3, Atg7 and Atg10 enzymes. Atg4 is an enzyme needed for the recycling of Atg8, i.e., its deconjugation, while Atg16 
is important component of the Atg12 Atg5 complex needed for its function. Furthermore, Atg16 is also important for the lipidation of Atg8 [17].

In $S$. cerevisiae, Atg8 is essential for autophagosome formation having roles in membrane expansion and autophagosome-lysosome fusion [17]. In H. sapiens, Atg8 has six homologs: MAP1LC3A (microtubuleassociated protein-1 light chain 3A), MAP1LC3B, MAP1LC3C, GABARAP (GABA $A$ receptor-associated protein), GABARAPL1 (GABARAP-like protein 1) and GABARAPL2 [32]. Atg8 homologs were proposed to be important in vesicle fusion which contributes to the growth of autophagosomal membranes [33, 34]. However, this hemifusion ability is not enough in vivo and SNAREs are required for the autophagosome formation [35]. Furthermore, Atg8 and its human homologs are hubs of interaction [36] since they have a central role in binding the receptors for the cargo selection during all types of selective autophagy.

Atg12 is conjugated to Atg5 similarly to Atg8 lipidation. Once conjugated, Atg12 Atg5 interacts with Atg16 forming a complex of $\sim 350 \mathrm{kDa}$ in yeast due to the oligomerization property of Atg16. One of the functions of Atg16 is to correctly localize the complex to the correct membrane. This complex is involved in Atg8 lipidation.

The structural characterization of the proteins and complexes involved in autophagy started with the conjugation systems [19] for which the mechanistical details were structurally elucidated in the last five years [37, 38, 39, 40, 41, 42, 43, 44, 45, 46, 47]. All these structural studies gave insights into how several components of the conjugation systems interact with each other and how the reactions occur. However, the conjugation reaction is not the only question about Atg8 and its homologs worth investigating. Since Atg8 and especially is human homologs are easy to purify [48], a large attention was given to its role in selective autophagy [36], [49], [50], [51].

\subsection{Phosphoinositide binding domains}

Cellular membranes are characterized by their lipid composition. Phosphoinositides (PIs), phosphorylated derivatives of phosphatidylinositol, have a particular role in cellular signaling and in correct localization of proteins at the cellular locations where they will perform their function. There are seven natural PIs defined by the combination of phsophorylations at positions 3, 4 or 5 on the inositol ring. They are specifically recognized by several domains such as FYVE (Fab1, YOTB, Vac1 and EEA1) [52], [53], PH (pleckstrin homology) [54], PX (Phox homology) [55], [56], C2 (conserved region-2 of protein kinase C) [57], PTB (phosphotyrosine binding) [58], GOLPH3 (Golgi phosphoprotein 3) [59], ANTH (AP180 N-terminal homology) [60], ENTH (epsin N-terminal homology) [61], FERM (4.1, ezrin, radixin, moiesin) [62], PDZ (postsynaptic density 95, disk large, zonula occludens) [63], Tubby [64] and PROPPIN ( $\beta$-propellers that bind phosphoinositides) [1, 30 ,

2]. Each domain has its specificity, e.g., FYVE binds only PI3P or promiscuity, e.g., PH domains are known to bind all the PIs except phosphatidyl-5-phosphate. Each known domain was structurally characterized in at least one model protein. Figure 1.2 gives an overview of the PI binding domains, their structures and PI specificity.

The PI binding domains have binding pockets lined with basic amino acids: arginine, lysine and/or histidine in order to bind the negatively charged PIs. When histidine is present, a histidine switch might be involved for the regulation of the binding. Making use of the ease of histidines to get protonated and deprotonated, according to the environmental $\mathrm{pH}$, the binding affinity can increase in acidic conditions. This regulation mechanism is used by the PH domain of GRP1 protein [65] and by the FYVE domain of EEA1 [66]. Usually, the PI affinity 


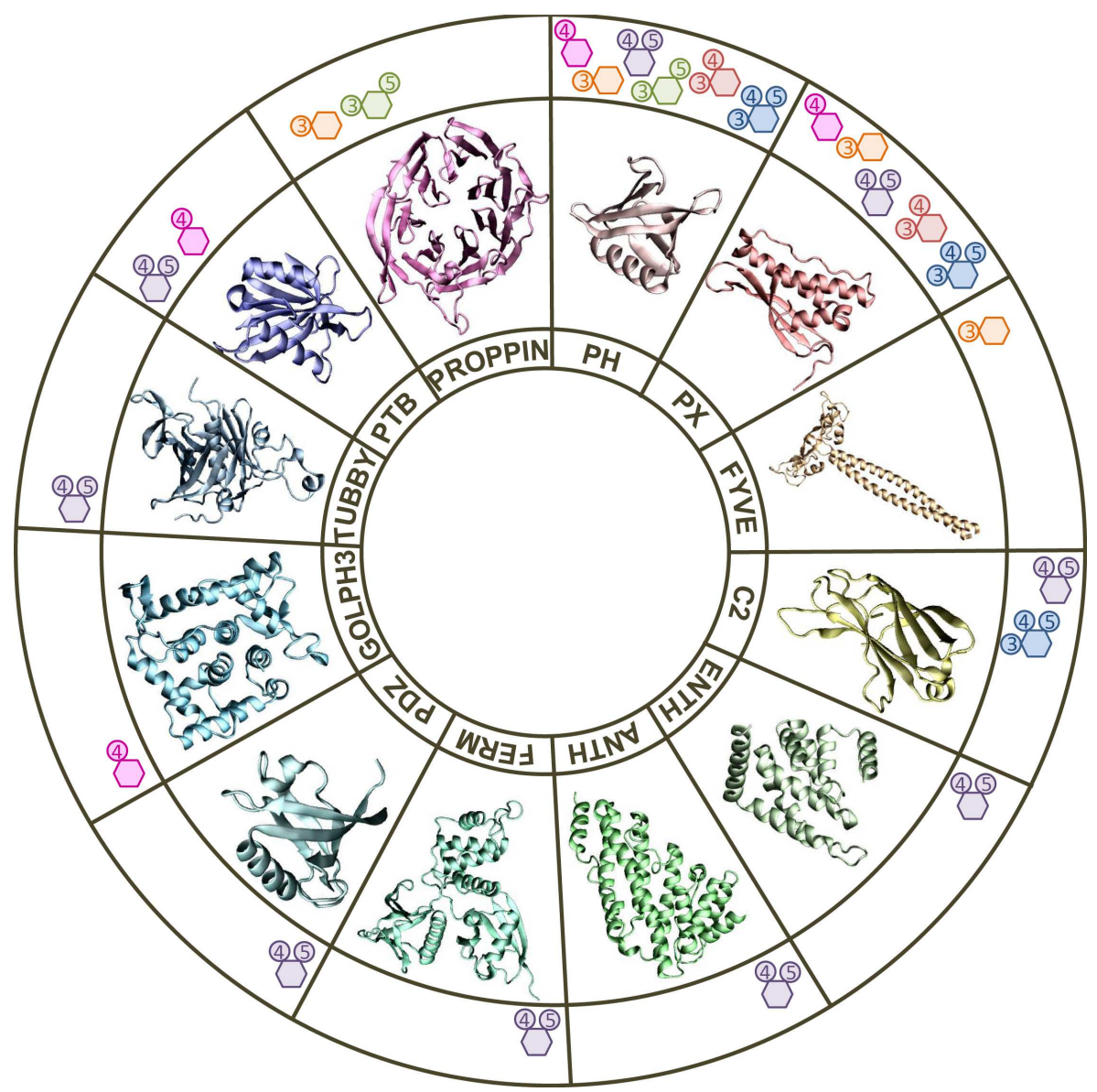

Figure 1.2: Phosphoinositide binding domains: structure and specificity.

There are seven natural phosphoinositides that give the characteristics of different cellular membranes. Each phospoinositide is recognized by specific phosphoinositide binding domains. Selected representatives of each of the known phosphoinositide binding domain: FYVE, PH, PX, C2, PTB, GOLPH3, ANTH, ENTH, FERM, PDZ, Tubby and PROPPINs are represented. Their ligand specificity is also shown.

of these domains is low. This is why, in order to have a tighter membrane binding, some domains are known to have another basic binding pocket for phosphatidylserine as in the case of $\mathrm{C} 2$ domain [67] or for phosphatidic acid as in the case of the PH domain [68] and PX domain [69]. Furthermore, it might be possible that two PI molecules bind to the same $\mathrm{C} 2$ domain [70]. Another mechanism to specifically increase affinity of one PI domain to a cellular membrane is domain oligomerization, e.g., the PH domain of dynamin [71] and the FYVE domain of EEA1 [52] or by combining the PI recruitment with the binding to another membrane attached protein, e.g., FAPPs bind both phosphatidylinositol-4 phosphate and ARF [72].

Beside the specific membrane attachment regulated through binding pockets that recognize PIs or other lipids or by physical interaction to membrane attached proteins, there are unspecific mechanisms for PI binding domains to be recruited to a cellular compartment. Electrostatic interactions are long range interactions. Through theoretical studies, it was shown that the electrical field created by the charges of a membrane, can orient $\mathrm{PH}$ domains in the right direction and bring them close to the membrane. Then, the PH domains bind the PI molecule [73]. Similarly, for the C2A domain of synaptotagmin 1 and 7, electrostatic docking was shown to be the recruitment mechanism. After electrostatical recruitment, synaptotagmin $7 \mathrm{C} 2 \mathrm{~A}$ domain, inserts a surface loop into the bilayer using its hydrophobic properties [74]. A similar interplay between electrostatic 
and hydrophobic interactions for loop insertion was previously reported for the PX domain of yeast Vam7p [75] and the FYVE domain of EEA1 [76]. Once a loop penetrates a membrane, the curvature of the membrane modifies due to increase in the surface of the outside leaflet [77]. This increase depends on the nature of the loop and depth of penetration. The coincidence detection of the membrane curvature and the PI is a recruitment mechanism for proteins such as sorting nexin-1 [78].

\subsection{Phosphoinositides in autophagy}

Phosphoinositide PI3P is a marker of the autophagosomal membranes [79]. The first proof that kinases and phosphatases are involved in the regulation of autophagy and that the lipid phosphorylation plays a crucial rule in autophagy signaling was offered by Per Seglen and Paul Gordon [80]. They have showed that 3-methyladenine inhibits autophagy. Later, it was shown that wortmannin also inhibits autophagy. Both 3methyladenine and wortmannin are inhibiting phosphatidyl 3-kinase (PI3K) [81]. However, rapamycin was shown to stimulate autophagy [82], which seemed contradicting the 3-methyladenine and wortmannin experiments, since all of them are blocking cellular signaling upstream of, or at, the TOR complex. However, in a Codogno-Meijer collaboration, it was shown that there are two different classes of phosphoinositides: phosphatidylinositol- $(3,4,5)$ triphosphate $\left(\operatorname{PIP}(3,4,5) \mathrm{P}_{3}\right)$ inhibits autophagy, while autophagy depends on PI3P [83]. Ohsumi's group has shown autophagy stimulation in yeast by rapamycin, suggesting that a similar signaling mechanism was conserved across species [84]. Later, after the identification of the autophagy molecular players, it was understood that the Vps34 complex I regulates PI3P signaling at the PAS with the help of Atg14.

In autophagy, there are several PI binding proteins:

- the PROPPIN family

- Atg20 contains a PX domain [85], [31]

- Atg24 contains a PX domain [85], [31]

- Atg26 contains a GRAM domain [86], [87]

- $\operatorname{Atg} 27[88]$

- ALFY (autophagy linked FYVE protein) contains a FYVE domain [89]

- FYCO1 (FYVE and coiled-coil domain containing protein) contains a FYVE domain [90]

- the transmembrane protein DFCP1 (double FYVE-containing protein 1) containing a FYVE domain [91]

- TECPR1 (Tectonin $\beta$-propeller repeat-containing protein) contains a PH domain [92]

\subsection{PROPPINs and their function}

PROPPINs are a PI binding family. In yeast autophagy, there are three PROPPIN paralogs: Atg18, Atg21 and Hsv2. The autophagy PROPPINS specifically bind PI3P and PI(3,5)P2 using a conserved FRRG motif 
[93, 94]. All three yeast PROPPINs seem to localize to a perivacuolar punctate structure and to the endosomes [95].

There are four human homologs of PROPPINs, the WIPI proteins (WD-40 repeat containing protein that interacts with PIs) [96]. It is hypothesized that WIPI1 and WIPI2 are the human homologs of Atg18, while WIPI3 and WIPI4 are the homologs of Hsv2. There are no human homologs for Atg21. The WIPI proteins are involved in a number of diseases [97] such as cancer, phospholipidosis and NBIA (neurodegeneration with brain iron accumulation). Some of the point mutations leading to illness are known [98, 99]. In C. elegans, a WIPI4 homolog, EPG-6, was reported to physically interact with Atg2. [100]. Furthermore, A. thaliana was reported to have eight PROPPIN homologs [101].

In S. cerevisiae, $\operatorname{Atg} 18$ is a $55 \mathrm{kDa}$ PROPPIN important in autophagy and in maintaining vacuolar morphology [102], [103], [104]. Atg18 is localized under both growing and starvation conditions in the cytosol, at the PAS and on the vacuolar membrane. Atg18 is needed for autophagosome formation, function which is done with the help with Atg2 and Atg9 [31], in the regulation of $\mathrm{PI}(3,5) \mathrm{P}_{2}$ synthesis at the vacuole [105] and in vesicular transport from the vacuoles to the Golgi [104]. Atg18 is required in macroautophagy, being one of the core eighteen protein, but also in the Cvt (cytoplasm to vacuole targeting) pathway for the maturation of Ape1 (amino peptidase 1), pexophagy in P. pastoris [103], [106] and PMN (piecemeal nucleophagy), microautophagy pathways in which parts of the nuclear membrane together with the nucleoplasm are engulfed by the vacuole [95]. For its autophagy related functions, Atg18 binds PI3P, while for the vacuolar morphology function, it binds $\mathrm{PI}(3,5) \mathrm{P}_{2}$.

Atg21 is a yeast PROPPIN involved in the Cvt pathway [107], [94] and PMN [95]. Furthermore, it was shown to be involved in pexophagy in P. pastoris [108] and P. angusta [109]. In the Cvt pathway, Atg21 was shown to function in the correct localization of Atg8 to the PAS [93], [110].

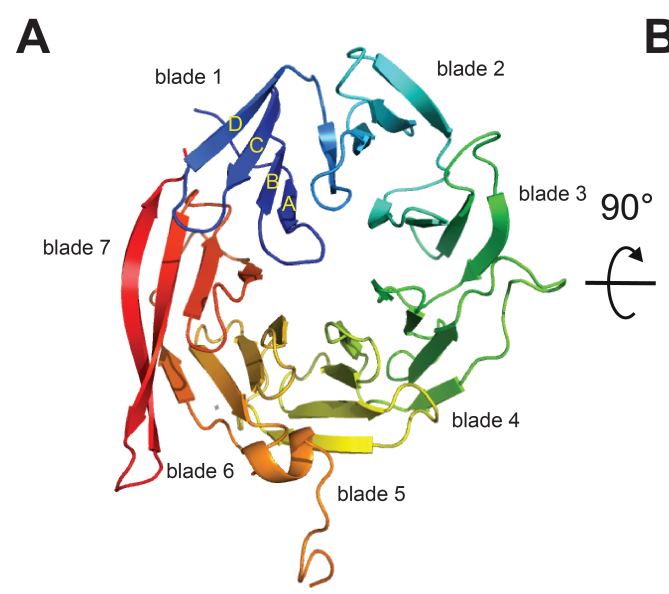

B C
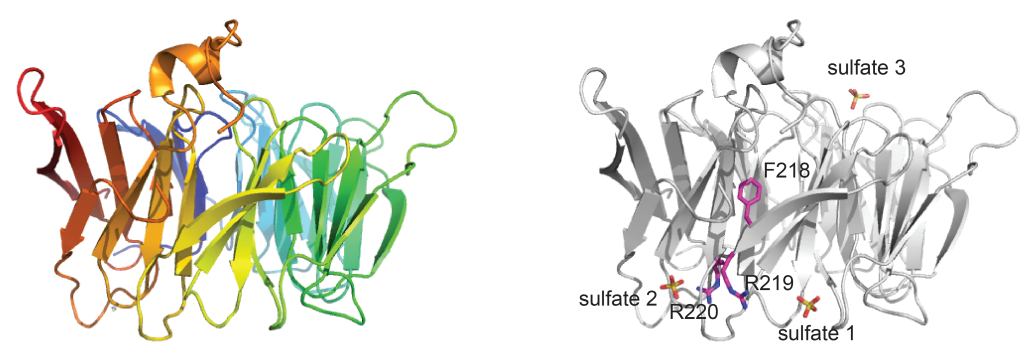

Figure 1.3: Structural details of KIHsv2, the first PROPPIN paralog structurally characterized.

Top (A) and side (B) view of the $\beta$-propeller of KlHsv2 (pdb accession number 4AV9 [1]). The cartoon representation is colored in rainbow colors with the $\mathrm{N}$-terminal represented in blue and the $\mathrm{C}$-terminal in red. The FRRG motif $(\mathrm{C})$ is essential for phosphoinositide binding. Its two arginines point towards two distinct binding pockets defined by crystallographic sulfates.

Hsv2 (homologous of the swollen vacuole protein 2), is the protein product of YGR233c gene [111]. It is important in the PMN pathway [95]. During the time course of this thesis, the structure of Hsv2 was determined in our laboratory [1] and in other groups [30], [2] in K. lactis (hereafter referred as KlHsv2) and K. marxianus. The Hsv2 structure (Figure 1.3A, B) presents a seven bladed beta-propeller. Each blade is formed by four anti- 
parallel $\beta$-strands connected by loop regions. These loops are not conserved among PROPPIN parologs from different species and have a variety of lengths. KlHsv2 has shorter loops when compared to its $S$. cerevisiae homolog (herafter referred as ScHsv2). It is hypothesized that these loops give the differences in binding partners and functions among the different paralogs, Atg18, Atg21 and Hsv2. In the $3 \AA$ structure of KlHsv2, there was not enough electronic density for the modeling of the loop connecting strand $\mathrm{C}$ and $\mathrm{D}$ of blade 6 (loop 6CD). However, this loop was visible in a $3.35 \AA$ structure. This loop is shown to be important for membrane binding [30]. Interestingly, in P. pastoris the phosphorylation of specific sites in the loop 6CD leads to Atg18 membrane binding, while phosphatates regulate its membrane detachment [106].

The structure allowed the characterization of the PI binding through the canonical FRRG motif (Figure 1.3. C). Intriguingly, the two arginines in this motif (R219 and R220) pointed towards two different pockets in which sulfates from the crystallization condition bound in the crystal structure. Sulfates are known to suggest the binding mode of phosphate functional groups in PIs [69], [112]. Mutagenesis of conserved residues in the region around these two sulfates revealed in both in vivo and in vitro experiments that there are two binding pockets for PI3P and $\mathrm{PI}(3,5) \mathrm{P}_{2}[1],[30]$.

\section{$1.6 \beta$-propellers are hubs of interaction}

PROPPINs are $\beta$-propellers or WD40 repeat domains. The WD40 repeat domain is one the most abundant interaction domains in eukaryotes. Being an old evolutionary domain, it can also be found in bacteria [113]. WD40 containing proteins function in signal transduction, cell division, cytoskeleton assembly, chemotaxis and RNA processing since they are stable $\beta$-propeller structures to which other proteins, nucleic acids or lipids can bind stably or reversibly.

The first structure of a $\beta$-propeller was determined for the $\mathrm{G}$ protein heterotrimer [114], [115]. The $\beta$ propeller structure is defined by the presence of several copies of WD40 repeats. In general, each repeat contains 44-60 residue units with a glycine - histidine (GH) dipeptide about 11-24 residues from its N-terminus and terminates with a tryptophan-aspartate (WD) doublet residues at the C-terminus [116]. Each of the repeat folds into a four-stranded anti-parallel beta-sheet. There can be in between four and nine of these $\beta$-sheets per WD40 domain protein. In general, the overall topology of the beta-propellers is such that the first two $\beta$-strands at the $\mathrm{N}$-terminal form a blade with the last two C-terminal $\beta$-strands. This is called a velcro closure. However, non-velcro topologies are known for Aip1p [117] and Hsv2 [1], [30], [2]. Moreover, in the case of Sec13, the structure shows an open propeller with six blades to which a seventh blade is contributed by the interaction partner, Sec16 or Sec31 [118].

In terms of interactions, WD40 proteins are platforms for multiple modes of interactions making them central to many cellular processes that need several molecular players to come together [119]. Autophagy is one of these processes in which eighteen core proteins work together to mature the PAS into an autophagosome. In yeast autophagy, PROPPINs are the only $\beta$-propellers involved. In mammalian autophagy, beside PROPPINs, there are Atg16 [120], ALFY [89], TECPR1 [92] and Ambra1 [121]. 


\subsection{Interaction partners of PROPPINs}

Atg 18 needs protein interaction partners that act synergistically with the PI binding to increase its membrane affinity. It has two main functions and according to these functions, it has different interactions partners. In autophagy, Atg18 interacts with Atg2, while for its function in vacuolar morphology it interacts with Vac7 [79]. Other important Atg18 interaction partners are:

- $\operatorname{Atg} 1$ [122]

- Atg9 [123, 31]

- Fab1, 1-phosphatidylinositol-3-phosphate 5-kinase, vacuolar membrane kinase that generates $\mathrm{PI}(3,5) \mathrm{P}_{2}$ [111]

- UBI4, ubiquitin [124]

- Vac14, involved in synthesis of $\mathrm{PI}(3,5) \mathrm{P}_{2}[125,126]$

- Vac17, phosphoprotein involved in vacuole inheritance [111, 125, 105]

- Pex13, peroxisomal importomer complex component; integral peroxisomal membrane protein required for docking and translocation of peroxisomal matrix proteins [127]

- Pho85, cyclin-dependent kinase involved in regulating the cellular response to nutrient levels and environmental conditions and progression through the cell cycle [122]

Furthermore, Atg21 physically interacts with Atg1 [122], Pho85 [122], TVP15, an integral membrane protein; localized to late Golgi vesicles along with the v-SNARE Tlg2p [128] and UBI4 [129]. Similar yeast high throughput screens that identified the interaction partners of Atg21, showed that Hsv2 directly interacts with Vps21, a Rab protein [130], Vam7, a SNARE protein [131], TVP15 [132] and UBI4 [129].

It can be observes that all PROPPINs bind ubiquitin UBI4. This is interesting to note since $\beta$-propellers are known to bind ubiquitin folds [124] and there are two ubiquitin-like proteins in autophagy, Atg12 and Atg8, which were not yet shown to directly interact with PROPPINs.

In the human autophagy network, WIPI2 was shown to interact with DnaJ chaperones and not interact with the Atg2 homologs, which are the interaction partners of WIPI4 [32]. Recently, Atg16L1 was shown to be an interaction partner of WIPI2b [133].

\subsubsection{The Atg2-Atg18 complex}

In S. cerevisiae, Atg2 (product of YNL242w gene [134]) is a hydrophilic protein of 1,592 amino acids with a molecular mass of $178 \mathrm{kDa}$. It has no known domain. In addition, Atg2 has no posttranslational modifications [135].

The first report of the YNL242w gene deletion mutant, identified Atg2 as important in the process of sporulation. Later, Atg2 was characterized as a peripheral membrane protein involved in the completion of the autophagosome [136, 135, 137]. Yeast cells that have a deletion of Atg2 lose their viability both in nutrient rich 
conditions and under starvation [135, 137]. Beside sporulation, Atg2 was shown to be important in autophagy [137], CVT pathway [136, 137, 135] and pexophagy [137].

The two most important interaction partners of Atg2 are Atg9 and Atg18. Atg2 co-immunoprecipitates with Atg9 which is the only transmembrane protein involved in autophagy [137], [135]. Atg9 is suggested to supply the PAS with lipids during its shuttling, helping in the expansion of PAS towards a mature autophagosome.

Another interaction partner of Atg2 is Atg18. The exact function of the Atg2-Atg18 complex is unknown. It was suggested to control the cycling of Atg9 between the PAS and a peripheral compartment that might be the ER. This would lead to the elongation of the PAS. One way to look into the function, would be to study the localization both in nutrient rich conditions and upon autophagy induction. Microscopy has shown that under both normal nutrient conditions and starvation, Atg2 is localized at the PAS and in the cytosol [137]. However, upon autophagy induction, more $\operatorname{Atg} 2$ is recruited to the PAS. Unfortunately, yeast is too small to map the exact localization of Atg2 by conventional microscopy techniques. However, using fluorescence microscopy in yeast cells having an enlarged selective cargo of autophagosomes, it was observed that the Atg2-Atg18 complex is localized at the edge of the isolation membranes in close proximity to the ER exit sites [138]. This observation is strengthened by the fact that in mammalian cells, the Atg18 homolog, WIPI2 is required at the omegasomes, ER-localized P3P-containing structures, for their progression into autophagosomes [96]. This is also true for C. elegans where the WIPI4 homolog, EPG-6 is required for omegasome maturation [100]. EPG-6 interacts with Atg2.

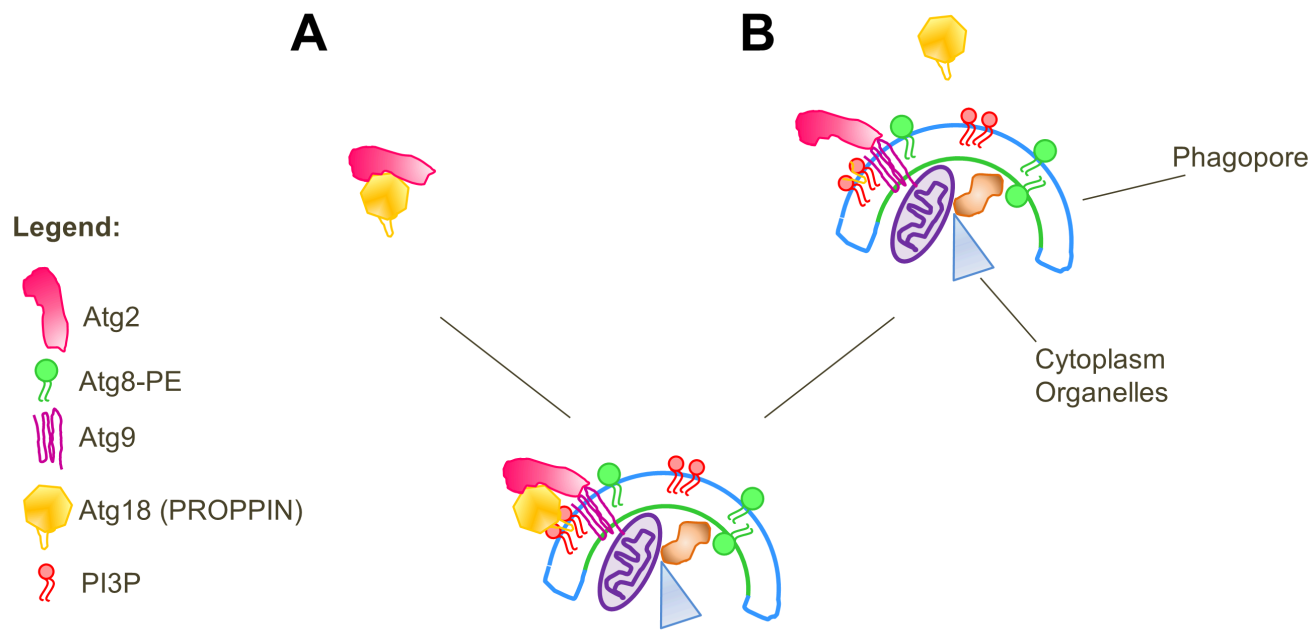

Figure 1.4: There are two working hypotheses for the recruitment of Atg2-Atg18 complex to the autophagic membranes. During autophagosome formation, the recruitment of Atg2-Atg18 is essential. (A) The Ohsumi laboratory [79] proposes a model in which the Atg2-Atg18 complex is formed in the cytosol and then recruited to the autophagic membranes. (B) The Reggiori laboratory [139] argues that Atg2 binds first to the pre-autophagosomal structure and subsequently, Atg18 binds to both PI3P and Atg2 forming a tight complex.

Dissecting the recruitment of the Atg2-Atg18 complex at the PAS, two hypotheses can be discussed. The molecular details of these hypotheses are schematically represented in Figure 1.4. Ohsumi's laboratory has shown that in Atg18 deficient cells, Atg2 fails to localize at the PAS [79]. This observation led to further characterization of the Atg2 and Atg18 interaction. They found that Atg2 and Atg18 constitutively form a cytosolic complex that is recruited to PAS by PI3P through the direct interaction of Atg18 with PI3P [79, 140]. Alternatively, in the laboratory of Reggiori, it was shown that Atg2 binds first at the PAS where it recruits Atg18 
[139]. This observation is based on the same experiment of Atg2 localization in Atg18 deficient cells done in Ohsumi lab. Contrary to the first results, in the case of the Reggiori laboratory, Atg2 can localize at the PAS in the absence of Atg18. However, a third laboratory [141] has shown the same results as the Ohsumi laboratory. Moreover, in this report [139], bimolecular fluorescence complementation (BiFC) was used to show that Atg2 and Atg18 interact only at the PAS.

However, Atg2-Atg18 complex localization to the PAS is not dependent alone to its ability to bind PI3P. Atg2 cannot localize to the PAS in Atg9 deficient cells, while Atg9 localizes to the PAS independent of the presence of Atg2 [137]. This means that Atg9 helps in recruiting Atg2-Atg18 complex to the PAS. Structural information on the interactions involved in this complex would settle the debate on the recruitment and function of the Atg2-Atg18 complex.

One powerful method to study domains important for conserved interactions is to check protein sequence conservation. Atg2 is little conserved among species and has no known domain. However, the $\mathrm{N}$ - and $\mathrm{C}$ - termini have some degree of conservation among species. From the studies of the Atg2 homolog in P. pastoris, Gsa11, it is known that C-terminal domain is needed for the localization to the PAS [137]. Moreover, the N-terminal was shown to be enough for PAS localization, but not enough for the autophagosome formation function [141]. Furthermore, glycine 83 was shown to be important for the localization of Atg2 to the PAS [135]. These facts indicate that the $\mathrm{N}$-terminal domain is the interaction domain with $\mathrm{Atg} 18$, while the $\mathrm{C}$-terminal domain is needed for recycling Atg9. The Atg2 interaction sites on Atg18 were recently characterized [2, 139] as being located on the opposite site of the propeller as the PI binding sites. Positions P72, R73 and loops 54-58, 90-94 and 121-123 in Atg18 were shown to be important for binding Atg2.

The information available up to now suggests that the Atg18-Atg2 complex may function in generating the high curvature at the growing sites on the PAS. The complex is not transported to the vacuole [137]. Indeed, the yeast phosphatase Ymr1 dephosphorylates PI3P before the autophagosome fuses with the vacuole, freeing the Atg2-Atg18 complex [142].

In the mammalian cells, there are two Atg2 paralogs, Atg2A and Atg2B. Human Atg2A was studied in yeast [141] where its N-terminal domain could localize at the PAS but would not function properly. Also, the human Atg2A would not interact with the yeast Atg9, while doing so with Atg18. Interestingly, in the human system, Atg2A/B are not shown to interact with Atg9 [32]. Both Atg2A and Atg2B are shown to function both in autophagosome formation and regulation of lipid droplet morphology and dispersion [143], [144]. In the human autophagy network [32], Atg2A is shown to interact with WIPI1, WIPI4, Atg2B, Atg8 family members and DnaJ chaperones, while Atg2B is shown to interact with WIPI4.

The human Atg2 proteins are not known to be involved in any disease, however, their paralogs, VPS13 family is involved in chorea acanthocytosis and Cohen syndrome [145]. In plants, the Atg2-Atg18 is shown to be important in powder mildew infection [146].

\subsubsection{Arabidopsis thaliana Atg18a-WRKY33 complex}

Autophagy is also conserved in plants where the vacuole uptakes cytosolic components. This was proven by morphological studies followed by identification of the molecular players based on sequence homology. In plants, autophagy occurs both under nutrient starvation and as a basal process during developmental stages, storage of proteins in the vacuole, senescence etc. 


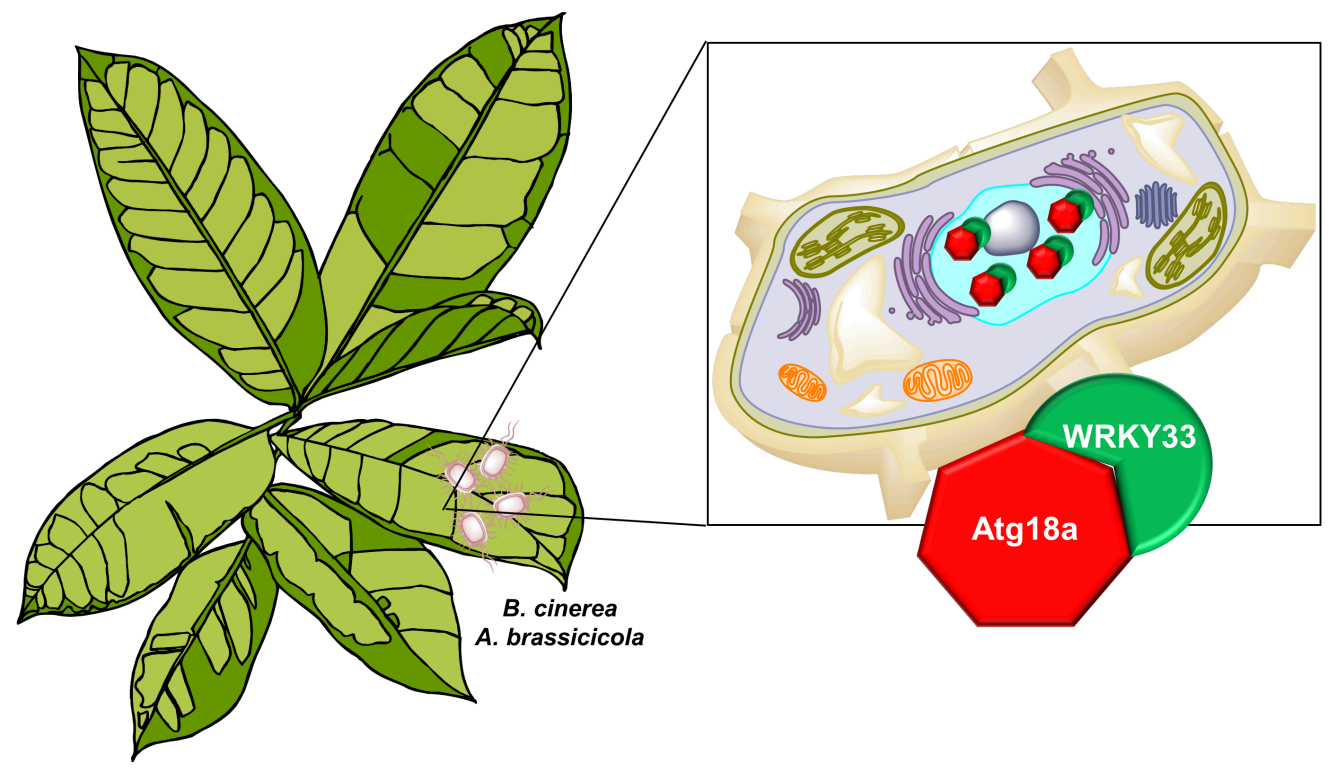

Figure 1.5: Importance of Atg18a-WRKY33 complex in the plant resistance to necrotrophic pathogens.

Upon infection with necrotrophic pathogens such as B. cinerea and A. brassicicola, Atg18a and transcription factor WRKY33 form a complex that is recruited to the nucleus. This complex is essential in the plant defense mechanism against necrotrohic pathogens.

Arabidopsis thaliana ATG18-like genes were identified upon comparison of the sequence of the S. cerevisiae ATG18 with the A. thaliana genome sequence. There are eight A. thaliana Atg18 proteins named AtAtg18a-AtAtg18h [101]. Phylogenetic analysis predicted that the AtAtg18s form three major clusters:

- AtATG18a, c, d, and e cluster with the yeast protein Hsv2

- AtAtg18b is most similar to yeast Atg18

- AtATG18f, g, and h form a separate clade

Similarly with human WIPI proteins, none of the eight plant Atg18 homologs clusters with the yeast Atg21.

Looking closer to AtAtg18a, under sucrose and starvation conditions, its transcription increases [101]. In addition, it is the only plant Atg18 upregulated in senescence and it may function in the response of plants to starvation. It is not required in normal nutrient-rich conditions, since AtATG18a RNAi plants are comparable to the wild type ones. Similarly, the plants lacking AtAtg18a are sensitive to oxidative stress, drought or salt [147, 148]. Furthermore, AtAtg2-AtAtg18a complex is important in the defense response to the powdery mildew fungus, G. cichoracearum, a biotrophic pathogen [146].

AtAtg18a was recently shown to interact with WRKY33 (AtWRKY33), a transcription factor required for resistance to necrotrophic pathogens [149]. Necrotrophic pathogens kill host cells before colonizing them. The host defends itself against these type of pathogens by using a system of multiple genes involved in jasmonate and ethylene signaling and synthesis of the phytoalexin camalexin [149]. However, there are indications that other pathways are also involved. These pathways might be interrelated with the WRKY33 pathway.

WRKY transcription factors [150] are DNA binding proteins that are regulating plant innate immunity, microbe- or pathogen-associated molecular pattern-triggered immunity and effector-triggered immunity. The defining feature of the WRKY proteins are the DNA binding motif defined by the amino acid sequence WRKY 
found at the N-terminal. WRKY proteins are mostly unstructured, nevertheless, the WRKY domain alone was structurally characterized as being a novel zinc-finger. It consists of a four-stranded $\beta$-sheet, Cys/His residues coordinating the zinc defining the zinc-binding pocket [151], [152].

When it comes to necrotrophic infections, both mutants in either AtAtg18a or AtWRKY33 show decreased resistance to $B$. cinerea and $A$. brassicicola. The interaction of these proteins was shown through yeast twohybrid screens and confirmed through co-immunoprecipitation. Moreover, AtAtg18a and AtWRKY33 interact in the nucleus through the C-terminal domain of AtWRKY33 [149]. The nuclear interaction was visualized through bimolecular fluorescence complementation.

This interaction opens interesting questions since this is the first instance of an autophagy protein present in the nucleus. How is it translocated? Does it bind any PI inside the nucleus? Interestingly, it was shown that PI3P is present in the plant nucleus [153]. How does it regulate WRKY33? For answering this question, the structural details of the AtAtg18a-AtWRKY33 interaction would be necessary.

\subsection{Aims}

The PROPPINs Atg18, Atg21 and Hsv2 are important in autophagy, Atg18 is one of the 18 core autophagic proteins. Their structures are predicted to be similar, however they have different functions in autophagic pathways. The details of how they perform their functions and what makes them different from each other are not known. Moreover, since they have different interaction partners, my goal was to get further knowledge on how PROPPINs interact with membranes by combining X-ray crystallography and biophysical and computational methods

The KlHsv2 structure was earlier determined in our group. Neither our nor the other published Hsv2 structures [30, 2] contained bound PI molecules. In order to get further insights into PI binding of PROPPINs on a molecular level, I performed docking studies. Another goal was to characterize the non-specific membrane binding of loop 6CD through coarse-grained and atomistic molecular dynamics simulations as described in Chapter 3 of this thesis.

In Chapter 4 of the thesis, I quantitatively characterized PI binding of the three PROPPIN paralogs from different yeast. The purified proteins were characterized using isothermal titration calorimetry (ITC), thermofluor and circular dichroism (CD) measurements.

Chapter 5 contains the structural characterization of P. angusta Atg18 (PaAtg18), as one of the last undetermined structures of the core autophagic complex. Atg 18 complexes are of great interest. The chosen interaction partners targets are Atg2 and AtWRKY33, which were cloned and expressed in this study. 


\section{Materials and Methods}

\subsection{Materials}

\subsubsection{Chemicals}

Reagent grade chemicals were purchased from the following companies: Fluka (Buchs, Switzerland), Merck (Darmstadt, Germany), Sigma-Aldrich (Steinheim/Seelze, Germany), Roth (Karlsruhe, Germany), Serva (Heidelberg, Germany), Roche (Basel, Switzerland). Chemicals used were of analytical purity and chemicals for crystallization were ultrapure quality. For crystallization, solutions from Emerald BioSystems, Hampton Research and Qiagen were used. Further chemicals are listed in table 2.1

Table 2.1: Chemicals

\begin{tabular}{ll}
\hline Chemical & Company \\
\hline HEPES & GERBU Biotechnik \\
complete EDTA-free, Protease inhibitor tablet & Roche \\
Ni-NTA Sepharose & GE Healthcare \\
Gluthatione Sepharose 4B & GE Healthcare \\
Strep-Tactin Sepharose & IBA \\
SYPRO Orange & Sigma \\
Nycodenz & Progen \\
Na-cholate & Sigma \\
Sephadex G-50 & Sigma \\
IANBD Amide & Invitrogen \\
\hline \hline
\end{tabular}

\subsubsection{Enzymes}

Enzymes were ordered from the companies stated in table 2.2 and used as recommended by the manufacturer. 
Table 2.2: Enzymes

\begin{tabular}{ll}
\hline Enzyme & Company \\
\hline Restriction enzymes & NEB \\
T4 DNA Ligase & NEB \\
Cre Recombinase & NEB \\
Thrombin & MP Biomedical \\
DNaseI & Applichem \\
Lysozyme & Roth \\
Proteinase K & Hampton Research \\
Trypsin & Hampton Research \\
Subtilisin & Hampton Research \\
\hline \hline
\end{tabular}

Thrombin was prepared in Tris/EDTA buffer (10 mM Tris pH 7.4, 1 mM EDTA) and mixed with an equal amount of glycerol.

\subsubsection{Lipids}

Lipids were ordered from the companies stated in table 2.3. They were shipped as powder and were dissolved in chloroform to the needed concentration and stored at $-20^{\circ} \mathrm{C}$.

Table 2.3: Lipids

\begin{tabular}{|c|c|c|c|}
\hline Lipid & Concentration & Order No. & Company \\
\hline PC: L- $\alpha$-phosphatidylcholine from egg, chicken & $10 / 25 \mathrm{mg} / \mathrm{ml}$ & $840051 \mathrm{C} / \mathrm{P}$ & Avanti Polar Lipids, Inc. \\
\hline PE: L- $\alpha$-phosphatidylethanolamine from brain, porcine & $25 \mathrm{mg} / \mathrm{ml}$ & $840022 \mathrm{P}$ & Avanti Polar Lipids, Inc. \\
\hline $\begin{array}{l}\text { 18:1 PI3P: 18:1 PtdIns3P: 1,2-dioleoyl-sn-glycero-3- } \\
\text { phospho-(1'-myoinositol-3'-phosphate) }\end{array}$ & $1 \mathrm{mg} / \mathrm{ml}$ & $850150 \mathrm{P}$ & Avanti Polar Lipids, Inc. \\
\hline $\begin{array}{l}\text { 18:1 PI(3,5)P } \mathbf{P}_{2}: \text { 1,2-dioleoyl-sn-glycero-3-phospho-(1'- } \\
\text { myo-inositol-3', 5'-bisphosphate) ammonium salt }\end{array}$ & $1 \mathrm{mg} / \mathrm{ml}$ & $850154 \mathrm{P}$ & Avanti Polar Lipids, Inc. \\
\hline $\begin{array}{l}\text { TR-PE: } \quad 1,2 \text {-dihexadecanoyl-sn-glycero-3- } \\
\text { phosphoethanolamine, triethylammonium salt }\end{array}$ & $1 \mathrm{mg} / \mathrm{ml}$ & $\mathrm{T}-1395 \mathrm{MP}$ & Invitrogen \\
\hline $\begin{array}{l}\text { 18:1 }(\Delta 9 \text {-Cis }) \text { DOPE: } 1,2 \text {-dioleoyl-sn-glycero-3- } \\
\text { phosphoethanolamine }\end{array}$ & & $850725 \mathrm{C}$ & Avanti Polar Lipids, Inc. \\
\hline $\begin{array}{l}\text { 18:1 ( } \Delta \text { 9-Cis }) \text { DOPC: 1,2-dioleoyl-sn-glycero-3- } \\
\text { phosphocholine }\end{array}$ & & $850375 \mathrm{C}$ & Avanti Polar Lipids, Inc. \\
\hline
\end{tabular}

\subsubsection{Kits}

All kits used in this study are summarized in table 2.4. They were used as recommended by the manufacturer. 
Table 2.4: Kits used in this study

\begin{tabular}{ll}
\hline Kit & Company \\
\hline Phusion High-Fidelity PCR Kit & NEB \\
NucleoSpin Plasmid kit & Macherey \& Nagel \\
NucleoSpin Extract II kit & Macherey \& Nagel \\
QIAquick PCR Purification kit & Qiagen \\
QIAquick Gel Extraction kit & Qiagen \\
NucleoBond PC100 & Macherey \& Nagel \\
NucleoBond Xtra & Macherey \& Nagel \\
Chaperone Plasmid Set & Takara Clontech \\
QuickChange Ligthning Site-Directed Muta- & Agilent Technologies (Stratagene) \\
genesis kit & \\
Western Lightening Plus-ECL & Perkin Elmer \\
ADDit - Additive Screen & Emerald BioSystems \\
pHat Buffer Block & Emerald BioSystems \\
\hline \hline
\end{tabular}

\subsubsection{Columns for chromatography}

All columns listed below in table 2.5 where used, as recommended by the manufacturer, in combination with an Äkta Purifier FPLC system (RT) or with the Äkta Prime FPLC $\left(4^{\circ} \mathrm{C}\right)$. For desalting the NBD-labeled proteins, PD-10 Desalting Columns (GE Healthcare) were used.

Table 2.5: Chromatography columns

\begin{tabular}{ll}
\hline Column & Company \\
\hline $1 \mathrm{ml} / 5 \mathrm{ml}$ His-Trap FF column & GE Healthcare \\
$5 \mathrm{~mL}$ Protino Ni-NTA column & Macherey \& Nagel \\
$5 \mathrm{ml}$ Strep-Trap column & GE Healthcare \\
$5 \mathrm{ml}$ GSTrap column & GE Healthcare \\
$5 \mathrm{~mL}$ Protino GST/4B Column & Macherey \& Nagel \\
HiLoad 16/60 Superdex 75 prep grade & GE Healthcare \\
\hline \hline
\end{tabular}

\subsubsection{Antibodies}

All antibodies used in this study are listed in table 2.6 . 
Table 2.6: Antibodies

\begin{tabular}{ll}
\hline Antibody & Company \\
\hline Ms mAb to GST & Abcam \\
Hsv2 (CGEPTRWELVRESWREL) & gift from Prof. M. Thumm \\
Hexa-Histidine tag (DIA900) & Dianova \\
murine Strep-tag II & IBA GmbH \\
Penta His HRP conjugate & Qiagen GmbH \\
rabbit polyclonal anti-HA & Sigma \\
goat polyclonal mouse IgG (HRP labeled) & BioRad Laboratories GmbH \\
goat polyclonal rabbit IgG (HRP labeled) & BioRad Laboratories GmbH \\
\hline \hline
\end{tabular}

\subsubsection{Buffers and media}

All buffers used in this study are listed in table 2.7, while media are summarized in table 2.8

Table 2.7: Buffers

\begin{tabular}{|c|c|}
\hline Buffer & Content \\
\hline PBS & $150 \mathrm{mM} \mathrm{NaCl}, 20 \mathrm{mM} \mathrm{Na}_{2} \mathrm{PO}_{4} \mathrm{pH} 7.4$ \\
\hline PBS-T & PBS buffer $+0.05 \%(\mathrm{v} / \mathrm{v})$ Tween 20 \\
\hline SDS-PAGE Running buffer 10x & $30.3 \mathrm{~g} / \mathrm{L}$ Tris, $144.6 \mathrm{~g} / \mathrm{L}$ Glycine, $10 \mathrm{~g} / \mathrm{L}$ SDS, pH 8.4 \\
\hline Transfer buffer & $2.9 \mathrm{~g} / \mathrm{L}(\mathrm{w} / \mathrm{v}$ ) Glycine, $5.8 \mathrm{~g} / \mathrm{L}(\mathrm{w} / \mathrm{v})$ Tris, $0.37 \mathrm{~g} / \mathrm{L}(\mathrm{w} / \mathrm{v})$ SDS, $20 \%$ (v/v) Methanol \\
\hline TAE 50x & $242 \mathrm{~g} / \mathrm{L}$ Tris, $57,1 \mathrm{ml} / \mathrm{L}$ glacial acetic acid, $100 \mathrm{ml} / \mathrm{L}$ of a $0.5 \mathrm{M}$ EDTA stock \\
\hline Lysis buffer & $\begin{array}{l}50 \mathrm{mM} \text { HEPES pH 7.5, } 500 \mathrm{mM} \mathrm{NaCl}, 20 \mathrm{mM} \text { imidazole, } 1 \mathrm{mM} \mathrm{MgCl}_{2} \text {, tablet of Roche protease inhibitors, } \\
\text { spatula of DNase I, spatula of lysozyme }\end{array}$ \\
\hline HisTrap buffer A & $50 \mathrm{mM}$ HEPES, $500 \mathrm{mM} \mathrm{NaCl}, 20 \mathrm{mM}$ imidazole, $\mathrm{pH} 7.5$ \\
\hline HisTrap buffer B & $50 \mathrm{mM}$ HEPES, $500 \mathrm{mM} \mathrm{NaCl}, 500 \mathrm{mM}$ imidazole, pH 7.5 \\
\hline StrepTrap/GSTrap buffer A & $50 \mathrm{mM}$ HEPES, $500 \mathrm{mM} \mathrm{NaCl}, \mathrm{pH} 7.0$ \\
\hline StrepTrap buffer B & $50 \mathrm{mM}$ HEPES, $500 \mathrm{mM} \mathrm{NaCl}, 2.5 \mathrm{mM}$ desthiobiotin, $\mathrm{pH} 7.0$ \\
\hline GSTrap buffer B & $50 \mathrm{mM}$ HEPES, $500 \mathrm{mM} \mathrm{NaCl}, 20 \mathrm{mM}$ gluthatione, $\mathrm{pH} 7.0$ \\
\hline Gel filtration buffer & $30 \mathrm{mM}$ HEPES, $300 \mathrm{mM} \mathrm{NaCl}, 1 \mathrm{mM}$ DTT, pH 7.0 \\
\hline Resolving gel buffer & 1.5 M Tris, $0.4 \%$ SDS, pH 8.8 \\
\hline Stacking gel buffer & 0.5 M Tris, $0.4 \%$ SDS, pH 6.6 \\
\hline SDS-PAGE buffer & $\begin{array}{l}9 \mathrm{~g} \text { SDS, } 30 \mathrm{~g} \text { glycerol, } 0.02 \mathrm{~g} \text { bromphenol blue, } 18.75 \mathrm{~mL} \text { of } 1 \mathrm{M} \text { Tris } \mathrm{pH} 6.8,90 \mathrm{~mL} \mathrm{H} 2 \mathrm{O} \text {; for use mix: } 9 \\
\text { parts of premix and } 1 \text { part beta-mercaptoethanol (end concentration } 3.3 \% \text { ) }\end{array}$ \\
\hline CD buffer & $30 \mathrm{mM} \mathrm{NaH}_{2} \mathrm{PO}_{4} / \mathrm{Na}_{2} \mathrm{HPO}_{4} \mathrm{pH} 7.4,150 \mathrm{mM} \mathrm{NaF}$ \\
\hline HP150 & $20 \mathrm{mM}$ HEPES $\mathrm{pH} 7.4,150 \mathrm{mM} \mathrm{KCl}$ \\
\hline B88 buffer & $20 \mathrm{mM}$ HEPES pH 6.8, $250 \mathrm{mM}$ sorbitol, $150 \mathrm{mM}$ KOAc, $5 \mathrm{mM} \mathrm{Mg}(\mathrm{OAc})_{2}$ \\
\hline
\end{tabular}

Table 2.8: Media

\begin{tabular}{|c|c|c|}
\hline Media & & \\
\hline \multirow{2}{*}{$\begin{array}{l}\text { Luria } \\
\text { (LB) }\end{array}$} & medium & $10 \mathrm{~g} / \mathrm{L}$ tryptone, $5 \mathrm{~g} / \mathrm{L}$ yeast extract, $10 \mathrm{~g} / \mathrm{L} \mathrm{NaCl}$ \\
\hline & & $18 \mathrm{~g} / \mathrm{L}$ of agar was added for plates \\
\hline
\end{tabular}


Table 2.8: Media

\begin{tabular}{|c|c|c|}
\hline Media & Content & \\
\hline $\begin{array}{l}\text { Terrific broth medium } \\
\text { (TB) }\end{array}$ & & 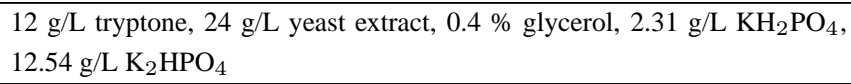 \\
\hline \multirow{5}{*}{ ZYM5052 } & $\mathrm{ZY}$ up to $1 \mathrm{~L}$ & $10 \mathrm{~g} / \mathrm{L} \mathrm{N}$-Z-Amine AS (Sigma) and $5 \mathrm{~g} / \mathrm{L}$ yeast extract-B (QBIOgene) \\
\hline & $20 \mathrm{~mL} 50 \times 5052$ & $\begin{array}{l}250 \mathrm{~g} / \mathrm{L}(\mathrm{w} / \mathrm{v}) \text { glycerol, } 25 \mathrm{~g} / \mathrm{L}(\mathrm{w} / \mathrm{v}) \text { glucose, } 100 \mathrm{~g} / \mathrm{L}(\mathrm{w} / \mathrm{v}) \text { a-lactose mono- } \\
\text { hydrate }\end{array}$ \\
\hline & $20 \mathrm{~mL} 50 \mathrm{xM}$ & $\begin{array}{l}222.5 \mathrm{~g} / \mathrm{L}(\mathrm{w} / \mathrm{v}) \mathrm{Na}_{2} \mathrm{HPO}_{4} \times 2 \mathrm{H}_{2} \mathrm{O}, 170 \mathrm{~g} / \mathrm{L}(\mathrm{w} / \mathrm{v}) \mathrm{KH}_{2} \mathrm{PO}_{4}, 134 \mathrm{~g} / \mathrm{L}(\mathrm{w} / \mathrm{v}) \text {, } \\
\mathrm{NH}_{4} \mathrm{Cl}, 35.5 \mathrm{~g} / \mathrm{L} \mathrm{Na}_{2} \mathrm{SO}_{4}\end{array}$ \\
\hline & $200 \mu \mathrm{L} \mathrm{1000x}$ trace metals mixture & $\begin{array}{l}50 \mathrm{mM} \mathrm{Fe}, 20 \mathrm{mM} \mathrm{Ca}, 10 \mathrm{mM} \mathrm{Mn}, 10 \mathrm{mM} \mathrm{Zn}, 2 \mathrm{mM} \mathrm{Co}, 2 \mathrm{mM} \mathrm{Cu}, 2 \mathrm{mM} \\
\mathrm{Ni}, 2 \mathrm{mM} \mathrm{Mo}, 2 \mathrm{mM} \mathrm{Se}, 2 \mathrm{mM} \mathrm{B}\end{array}$ \\
\hline & $2 \mathrm{~mL} 1 \mathrm{M} \mathrm{MgSO}_{4}$ & \\
\hline \multirow[t]{5}{*}{ ZYM505 } & ZY up to $1 \mathrm{~L}$ & $10 \mathrm{~g} / \mathrm{L} \mathrm{N}-\mathrm{Z}$-Amine AS (Sigma) and $5 \mathrm{~g} / \mathrm{L}$ yeast extract-B (QBIOgene) \\
\hline & $10 \mathrm{~mL} 100 \times 505$ & $500 \mathrm{~g} / \mathrm{L}(\mathrm{w} / \mathrm{v})$ glycerol, $55 \mathrm{~g} / \mathrm{L}(\mathrm{w} / \mathrm{v})$ glucose \\
\hline & $20 \mathrm{~mL} 50 \mathrm{xM}$ & $\begin{array}{l}222.5 \mathrm{~g} / \mathrm{L}(\mathrm{w} / \mathrm{v}) \mathrm{Na}_{2} \mathrm{HPO}_{4} \times 2 \mathrm{H}_{2} \mathrm{O}, 170 \mathrm{~g} / \mathrm{L}(\mathrm{w} / \mathrm{v}) \mathrm{KH}_{2} \mathrm{PO}_{4}, 134 \mathrm{~g} / \mathrm{L}(\mathrm{w} / \mathrm{v}), \\
\mathrm{NH}_{4} \mathrm{Cl}, 35.5 \mathrm{~g} / \mathrm{L} \mathrm{Na}_{2} \mathrm{SO}_{4}\end{array}$ \\
\hline & $200 \mu \mathrm{L} 1000 \mathrm{x}$ trace metals mixture & $\begin{array}{l}50 \mathrm{mM} \mathrm{Fe}, 20 \mathrm{mM} \mathrm{Ca}, 10 \mathrm{mM} \mathrm{Mn}, 10 \mathrm{mM} \mathrm{Zn}, 2 \mathrm{mM} \mathrm{Co}, 2 \mathrm{mM} \mathrm{Cu}, 2 \mathrm{mM} \\
\mathrm{Ni}, 2 \mathrm{mM} \mathrm{Mo}, 2 \mathrm{mM} \mathrm{Se}, 2 \mathrm{mM} \mathrm{B}\end{array}$ \\
\hline & $2 \mathrm{~mL}_{1} \mathrm{M} \mathrm{MgSO}_{4}$ & \\
\hline \multirow[t]{6}{*}{ MD5052 } & $50 \mathrm{~mL}$ aspartate & \\
\hline & $20 \mathrm{~mL} 50 \times 5052$ & $\begin{array}{l}250 \mathrm{~g} / \mathrm{L}(\mathrm{w} / \mathrm{v}) \text { glycerol, } 25 \mathrm{~g} / \mathrm{L}(\mathrm{w} / \mathrm{v}) \text { glucose, } 100 \mathrm{~g} / \mathrm{L}(\mathrm{w} / \mathrm{v}) \alpha \text {-lactose mono- } \\
\text { hydrate }\end{array}$ \\
\hline & $20 \mathrm{~mL} 50 \mathrm{xM}$ & $\begin{array}{l}222.5 \mathrm{~g} / \mathrm{L}(\mathrm{w} / \mathrm{v}) \mathrm{Na}_{2} \mathrm{HPO}_{4} \times 2 \mathrm{H}_{2} \mathrm{O}, 170 \mathrm{~g} / \mathrm{L}(\mathrm{w} / \mathrm{v}) \mathrm{KH}_{2} \mathrm{PO}_{4}, 134 \mathrm{~g} / \mathrm{L}(\mathrm{w} / \mathrm{v}), \\
\mathrm{NH}_{4} \mathrm{Cl}, 35.5 \mathrm{~g} / \mathrm{L} \mathrm{Na} \mathrm{SO}_{4}\end{array}$ \\
\hline & $200 \mu \mathrm{L} \mathrm{1000x}$ trace metals mixture & $\begin{array}{l}50 \mathrm{mM} \mathrm{Fe}, 20 \mathrm{mM} \mathrm{Ca}, 10 \mathrm{mM} \mathrm{Mn}, 10 \mathrm{mM} \mathrm{Zn}, 2 \mathrm{mM} \mathrm{Co}, 2 \mathrm{mM} \mathrm{Cu}, 2 \mathrm{mM} \\
\mathrm{Ni}, 2 \mathrm{mM} \mathrm{Mo}, 2 \mathrm{mM} \mathrm{Se}, 2 \mathrm{mM} \mathrm{B}\end{array}$ \\
\hline & $2 \mathrm{~mL} 1 \mathrm{M} \mathrm{MgSO}_{4}$ & \\
\hline & Autoclaved $\mathrm{dH}_{2} \mathrm{O}$ up to $1 \mathrm{~L}$ & \\
\hline \multirow[t]{2}{*}{ SD-ura } & $3.5 \mathrm{~g} \mathrm{~S}$-ura powder & $\begin{array}{l}25.1 \mathrm{~g} \text { yeast nitrogen base without amino acids and without ammonium sul- } \\
\text { fate, } 75.4 \mathrm{~g} \text { Ammonium sulfate, } 450 \mathrm{mg} \text { Isoleucine, } 2.25 \mathrm{~g} \text { Valine, } 300 \mathrm{mg} \\
\text { Adenine, } 300 \mathrm{mg} \text { Arginine, } 300 \mathrm{mg} \text { Histidine, } 450 \mathrm{mg} \text { Leucine, } 450 \mathrm{mg} \text { Ly- } \\
\text { sine, } 300 \mathrm{mg} \text { Methionine, } 750 \mathrm{mg} \text { Phenylalanine, } 300 \mathrm{mg} \text { Tryptophan, } 450 \\
\text { mg Tyrosine }\end{array}$ \\
\hline & 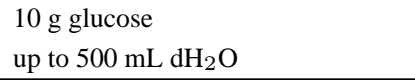 & \\
\hline \multirow[t]{3}{*}{$3 \mathrm{XYP}+6 \%$ galactose } & & $30 \mathrm{~g}$ yeast extract, $60 \mathrm{~g}$ peptone; fill up to $700 \mathrm{~mL} \mathrm{dH} 2 \mathrm{O}$ \\
\hline & & $60 \mathrm{~g}$ galactose in $300 \mathrm{~mL}$ dH2O; autoclave \\
\hline & & after autoclaving, mix the two solutions \\
\hline \multirow[t]{3}{*}{$\begin{array}{l}\mathrm{YP}+3 \% \text { glycerol }+2 \% \\
\text { ethanol }+2 \% \text { galactose }\end{array}$} & & $\begin{array}{l}10 \mathrm{~g} \text { yeast extract, } 20 \mathrm{~g} \text { peptone, } 30 \mathrm{~mL} \text { glycerol; up to } 880 \mathrm{~mL} \mathrm{dH} 2 \mathrm{O} \text {; } \\
\text { autoclave }\end{array}$ \\
\hline & & $20 \mathrm{~g}$ galactose in $100 \mathrm{~mL} \mathrm{dH} 20$; autoclave \\
\hline & & mix after autclaving and add $20 \mathrm{~mL}$ ethanol \\
\hline \multirow[t]{2}{*}{ SOC } & & $2 \%$ tryptone, $0.5 \%$ yeast extract, $10 \mathrm{mM} \mathrm{NaCl}, 2.5 \mathrm{mM} \mathrm{KCl}$ \\
\hline & & after autoclaving add $10 \mathrm{mM} \mathrm{MgCl}_{2}, 10 \mathrm{mM} \mathrm{Mg}_{2} \mathrm{SO}_{4} ; 20 \mathrm{mM}$ glucose \\
\hline
\end{tabular}

\section{Minimal media with selenomethionine}

11 culture consists of:

$200 \mathrm{ml} 5 \mathrm{x}$ M9 stock solution (15 g/l (w/v) $\left.\mathrm{KH}_{2} \mathrm{PO}_{4}, 5 \mathrm{~g} / \mathrm{l}(\mathrm{w} / \mathrm{v}) \mathrm{NH}_{4} \mathrm{Cl}, 2.5 \mathrm{~g} / \mathrm{l}(\mathrm{w} / \mathrm{v}) \mathrm{NaCl}\right)$

$800 \mathrm{ml}$ autoclaved water

$1 \mathrm{ml}$ of $1 \mathrm{M} \mathrm{MgSO}_{4}$ (autoclaved)

$20 \mathrm{ml} 20 \%$ glucose (w/v) (sterile filtered)

$100 \mu 1$ of $0.5 \%(\mathrm{w} / \mathrm{v})$ thiamine vitamin (sterile filtered) 
$1 \mathrm{ml}$ of $4.2 \mathrm{~g} / \mathrm{l}(\mathrm{w} / \mathrm{v}) \mathrm{FeIISO}_{4}$ (sterile filtered)

For inoculation an over night culture was harvested and spun down at low speed and washed with in M9 media to remove all full media components. The culture in minimal media was grown until an OD of 0.3 at $600 \mathrm{~nm}$. At this point solid amino acids were added:

$100 \mathrm{mg} / \mathrm{l}(\mathrm{w} / \mathrm{v})$ L-Lysine

$100 \mathrm{mg} / \mathrm{l}(\mathrm{w} / \mathrm{v})$ L-Phenylalanine

$100 \mathrm{mg} / \mathrm{l}(\mathrm{w} / \mathrm{v})$ L-Threonine

$50 \mathrm{mg} / \mathrm{l}(\mathrm{w} / \mathrm{v})$ L-Isoleucine

$50 \mathrm{mg} / \mathrm{l}(\mathrm{w} / \mathrm{v})$ L-Leucine

$50 \mathrm{mg} / \mathrm{l}(\mathrm{w} / \mathrm{v}) \mathrm{L}$-Valine

$50 \mathrm{mg} / \mathrm{l}(\mathrm{w} / \mathrm{v})$ L-Selenomethionine

About 15 min after amino acid addition the expression was started with $1 \mathrm{mM}$ IPTG.

\subsubsection{Antibiotics}

The following antibiotics were prepared as 1000x stock solutions. Ampicillin, gentamycin and kanamycin were prepared in deionized water, chloramphenicol powder was resuspended in $70 \%$. All solutions were filter sterilized and stored at $-20^{\circ} \mathrm{C}$.

Ampicillin $(100 \mu \mathrm{g} / \mathrm{ml}(\mathrm{w} / \mathrm{v}))$

Kanamycin $(30 \mu \mathrm{g} / \mathrm{ml}(\mathrm{w} / \mathrm{v}))$

Gentamycin $(20 \mu \mathrm{g} / \mathrm{ml}(\mathrm{w} / \mathrm{v}))$

Chloramphenicol $(35 \mu \mathrm{g} / \mathrm{ml}(\mathrm{w} / \mathrm{v}))$

\subsubsection{Yeast and bacterial strains}

E. coli BW23474 - cloning strain for plasmids with $\mathrm{R} 6 \mathrm{~K} \gamma$ origin

E. coli $\mathrm{DH} 5 \alpha$ - standard cloning strain

E. coli XL1-blue - standard cloning strain

E. coli $\mathrm{BL21}(\mathrm{DE} 3)$ - expression strain

E. coli Rosetta2(DE3)pLysS - expression strain

E. coli ArcticExpress(DE3) - expression strain

S. cerevisiae Y258 - expression strain

\subsubsection{DNA constructs}

In the following table 2.9 all used plasmids are stated. 
Table 2.9: DNA constructs generated and used in this study

\begin{tabular}{|c|c|c|c|c|c|c|}
\hline Vector & Gene & $\begin{array}{l}\text { Affinity } \\
\text { tag }\end{array}$ & $\begin{array}{l}\text { Cleavage } \\
\text { sites }\end{array}$ & $\begin{array}{l}\text { Cloning } \\
\text { sites }\end{array}$ & Resistance & Source \\
\hline pET-28(a)+ & & 6xHis & thrombin & & Kan & Novagen \\
\hline pETM-20 & & $6 \mathrm{xHis}$ & TEV & & Amp & EMBL \\
\hline pETM-41 & & $6 \mathrm{xHis}$ & TEV & & Kan & EMBL \\
\hline pETM-60 & & $6 \mathrm{xHis}$ & TEV & & Kan & EMBL \\
\hline pBADM-41(+) & & $6 \mathrm{xHis}$ & TEV & & Amp & EMBL \\
\hline pBADM-60(+) & & $6 \mathrm{xHis}$ & TEV & & Amp & EMBL \\
\hline pGex-4T-1 & & GST & thrombin & & Amp & \\
\hline pAce1 & & & & & Amp & I. Berger \\
\hline pAce1-N-His & & $6 \mathrm{xHis}$ & thrombin & & Amp & A. Scacioc \\
\hline pAce1-N-StrepII & & StrepII & thrombin & & Amp & A. Scacioc \\
\hline $\mathrm{pDk}$ & & & & & Kan & I. Berger \\
\hline pDk-StrepII & & StrepII & thrombin & & Kan & A. Scacioc \\
\hline pGex-6P-1 & ScAtg18 & GST & Prescission & & Amp & M. Thumm \\
\hline pGex-4T-1 & ScAtg21 & GST & thrombin & & Amp & M. Thumm \\
\hline pET-28a(+) & PaAtg18 & $6 \mathrm{xHis}$ & thrombin & NdeI/XhoI & Kan & R. Busse \\
\hline pET-28a(+) & KlAtg21 & $6 \mathrm{xHis}$ & thrombin & NdeI/XhoI & Kan & R. Busse \\
\hline pAce1-N-His & CtAtg18 & $6 \mathrm{xHis}$ & thrombin & NdeI/XhoI & Amp & O. Yagensky \\
\hline pETM-20 & CtAtg18 & $6 \mathrm{xHis}$ & TEV & NotI/NcoI & Amp & O. Yagensky \\
\hline pETM-41 & CtAtg18 & $6 \mathrm{xHis}$ & TEV & NotI/NcoI & Kan & O. Yagensky \\
\hline pETM-60 & CtAtg18 & $6 \mathrm{xHis}$ & TEV & NotI/NcoI & Kan & O. Yagensky \\
\hline pBADM-41(+) & CtAtg18 & $6 \mathrm{xHis}$ & TEV & $\mathrm{Ncol} / \mathrm{XhoI}$ & Amp & O. Yagensky \\
\hline pBADM-60(+) & CtAtg18 & $6 \mathrm{xHis}$ & TEV & $\mathrm{NcoI} / \mathrm{XhoI}$ & Amp & O. Yagensky \\
\hline pGex-4T-1 & CtAtg18 & GST & thrombin & BamHI/XhoI & Amp & O. Yagensky \\
\hline pGex-4T-1 & ScHsv2 & GST & thrombin & BamHI/XhoI & Amp & M. Thumm \\
\hline pGex-4T-1 & ScHsv2(R264A) & GST & thrombin & BamHI/XhoI & Amp & R. Busse \\
\hline pGex-4T-1 & ScHsv2(H294A) & GST & thrombin & BamHI/XhoI & Amp & R. Busse \\
\hline pET-28a(+) & PaAtg18(no cys, S51C) & $6 \mathrm{xHis}$ & thrombin & NdeI/XhoI & Kan & A. Scacioc \\
\hline pET-28a(+) & PaAtg18(no cys, S81C) & $6 \mathrm{xHis}$ & thrombin & NdeI/XhoI & Kan & A. Scacioc \\
\hline pET-28a(+) & PaAtg18(no cys, C113) & $6 \mathrm{xHis}$ & thrombin & NdeI/XhoI & Kan & A. Scacioc \\
\hline pET-28a(+) & PaAtg18(no cys, S157C) & $6 \mathrm{xHis}$ & thrombin & NdeI/XhoI & Kan & A. Scacioc \\
\hline pET-28a(+) & PaAtg18(no cys, S448C) & $6 \mathrm{xHis}$ & thrombin & NdeI/XhoI & Kan & A. Scacioc \\
\hline pET-28a(+) & PaAtg18(no cys, S459C) & $6 \mathrm{xHis}$ & thrombin & Ndel/XhoI & Kan & A. Scacioc \\
\hline \multirow[t]{2}{*}{ BG1805 } & ScAtg2 & $6 \mathrm{xHis}$ & $3 \mathrm{C}$ & & Amp & Thermo Scientific \\
\hline & & & & & & Open Biosystems \\
\hline pET-28a(+) & ScAtg2(1-307) & $6 \mathrm{xHis}$ & thrombin & NdeI/XhoI & Kan & S. David \\
\hline pET-28a(+) & ScAtg2(1-327) & $6 \mathrm{xHis}$ & thrombin & NdeI/XhoI & Kan & S. David \\
\hline pET-28a(+) & ScAtg2(1-354) & $6 \mathrm{xHis}$ & thrombin & NdeI/XhoI & Kan & S. David \\
\hline pET-28a(+) & ScAtg2(1-157) & $6 \mathrm{xHis}$ & thrombin & NdeI/XhoI & Kan & S. David \\
\hline pET-28a(+) & ScAtg2(1-229) & $6 \mathrm{xHis}$ & thrombin & NdeI/XhoI & Kan & S. David \\
\hline pET-28a(+) & ScAtg2(1-289) & $6 \mathrm{xHis}$ & thrombin & NdeI/XhoI & Kan & S. David \\
\hline pET-28a(+) & ScAtg2(1-189) & $6 \mathrm{xHis}$ & thrombin & NdeI/XhoI & Kan & S. David \\
\hline pAce1-N-His & CtAtg2(1-168) & $6 \mathrm{xHis}$ & thrombin & NdeI/XhoI & Amp & O. Yagensky \\
\hline pDk-StrepII & CtAtg2(1-168) & StrepII & thrombin & NdeI/XhoI & Kan & O. Yagensky \\
\hline pBADM-41(+) & CtAtg2(1-161) & $6 \mathrm{xHis}$ & TEV & $\mathrm{Ncol} / \mathrm{XhoI}$ & Amp & S. David \\
\hline pBADM-41(+) & CtAtg2(1-168) & $6 \mathrm{xHis}$ & TEV & NcoI/XhoI & Amp & S. David \\
\hline pBADM-41(+) & CtAtg2(1-279) & $6 \mathrm{xHis}$ & TEV & Ncol/XhoI & Amp & S. David \\
\hline pBADM-41(+) & CtAtg2(1-183) & $6 \mathrm{xHis}$ & TEV & Ncol/XhoI & Amp & S. David \\
\hline pBADM-41(+) & CtAtg2(1-206) & $6 \mathrm{xHis}$ & TEV & NcoI/XhoI & Amp & S. David \\
\hline pBADM-41(+) & CtAtg2(1-218) & $6 \mathrm{xHis}$ & TEV & Ncol/XhoI & Amp & S. David \\
\hline pBADM-41(+) & CtAtg2(1-266) & $6 \mathrm{xHis}$ & TEV & $\mathrm{NcoI} / \mathrm{XhoI}$ & Amp & S. David \\
\hline pBADM-41(+) & CtAtg2(1-270) & $6 \mathrm{xHis}$ & TEV & $\mathrm{NcoI} / \mathrm{XhoI}$ & Amp & S. David \\
\hline pBADM-41(+) & CtAtg2(1-244) & $6 \mathrm{xHis}$ & TEV & NcoI/XhoI & Amp & S. David \\
\hline pAce1-N-His & AtAtg18a & $6 \mathrm{xHis}$ & thrombin & NdeI/XhoI & Amp & A. Scacioc \\
\hline pAce1-N-StrepII & AtWRKY33 & StrepII & thrombin & NdeI/XhoI & Amp & A. Scacioc \\
\hline
\end{tabular}

\subsubsection{Oligonucleotides}

Oligonucleotides were ordered from Sigma-Genosys and purchased through Sigma Aldrich Chemie GmbH (Steinheim, Germany) or Eurofins Genomics (Ebersberg, Germany). The Tm of the PCR primers was calculated using http://eu.idtdna.com/analyzer/Applications/OligoAnalyzer/Default.aspx. All oligonucleotides used in this study are listed in table 2.10 
Table 2.10: Oligonucleotides ordered especially for this study

\begin{tabular}{|c|c|c|}
\hline Name & Sequence 5'-3' & $\begin{array}{l}\mathrm{Tm}\left({ }^{\circ} \mathrm{C}\right) \text { matching } \\
\text { (full) sequence }\end{array}$ \\
\hline ctAtg18_NcoI_f & AGTCGTCCATGGCCGCGACTTTAAACTATGTCAC & $59.3(65.1)$ \\
\hline ctAtg18_BamHI_f & CCAGTAGGATCCATGGCCGCGACTTTAAACTATGTCAC & $59.3(65.5)$ \\
\hline ctAtg18_XhoI_r & AGTCGTCTCGAGTTATTACACTCCATAAGCAC & $55.1(60.4)$ \\
\hline ctAtg18_NotI_r & AGTCGTGCGGCCGCCTCGAGTTATTACACTCCATAAGC & $52.9(68.6)$ \\
\hline CtAtg2_1_168_NdeI_f & TCTATACATATGGCGTCCTTTTTCCAGTC & $55.5(58)$ \\
\hline CtAtg2_1_168_XhoI_r & ATCACTCTCGAGTTATTATATACTCGCCCCTAAATCTTG & $51.5(60.7)$ \\
\hline Ct Atg2 1_168_NdeI_f & TCTATACATATGGCGTCCTTTTTCCAGTC & $55.5(58)$ \\
\hline Ct Atg2 1_218_XhoI_r & ATCACTCTCGAGTTAAGGGACCTCAACATCGCCCTG & $61.6(66.4)$ \\
\hline Ct Atg2 1_244_XhoI_r & ATCACTCTCGAGTTACGTCACACCCTCAACATTAATAC & $53.6(62.4)$ \\
\hline Ct Atg2 1_279_XhoI_r & ATCACTCTCGAGTTAGCAGCGCGGATATTGTTCAG & $56.8(64.5)$ \\
\hline Sc Atg18_Nco_f & TCTATACCATGGAGATGAGCGATAGCAGCCCAACC & $61.3(65.4)$ \\
\hline Sc Atg18_XhoI_r & ATCACTCTCGAGTTAGTCCATCAAAATCGAATATTGAG & $49.9(60.2)$ \\
\hline Sc Atg18_NdeI_f & TCTATACATATGAGCGATAGCAGCCCAACC & $59.9(60.9)$ \\
\hline Sc Atg18_NotI_r & ATCACTGCGGCCGCTTAGTCCATCAAAATCGAATATTGAG & $49.9(63.3)$ \\
\hline Sc Atg2 1_NdeI_f & TCTATACATATGGCATTTTGGTTACCTC & $50.4(54.8)$ \\
\hline Sc Atg2 1_137_XhoI_r & ATCACTCTCGAGTTAAGAGCTAATGTCATCTTCTTTG & $55.6(60.2)$ \\
\hline Sc Atg2 1_354_XhoI_r & ATCACTCTCGAGTTAGCTTTCATTGGCATCCAAATGTG & $53.5(63.5)$ \\
\hline Sc Atg2 1_189_XhoI_r & ATCACTCTCGAGTTACACTATGAAACGTATGGTAACGTC & $53.5(62.1)$ \\
\hline Sc Atg2 1_209_XhoI_r & ATCACTCTCGAGTTAAATAAGTTGTATGCTTTCTAG & $45.4(57.9)$ \\
\hline Sc Atg2 1_229_XhoI_r & ATCACTCTCGAGTTATTGTATTGAGGAAATGGTG & $47.3(59.4)$ \\
\hline Sc Atg2 1_269_XhoI_r & ATCACTCTCGAGTTATGATTGCTCTTCCATGGCGTC & $60.1(65.4)$ \\
\hline Sc Atg2 1_289_XhoI_r & ATCACTCTCGAGTTACTTGCACTTATCATTCTCTTG & $49.1(60.4)$ \\
\hline Sc Atg2 1_307_XhoI_r & ATCACTCTCGAGTTACGATGATAAACCCTTAAAAGC & $49.4(60.6)$ \\
\hline Sc Atg2 1_320_XhoI_r & ATCACTCTCGAGTTAAATATCAATAACAATATTAGACATTC & $45.9(60.6)$ \\
\hline Sc Atg2 1_327_XhoI_r & ATCACTCTCGAGTTATATCGCTAAATGAACATCC & $46.9(59.1)$ \\
\hline Sc Atg2 1_349_XhoI_r & ATCACTCTCGAGTTACAAATGTGTAACAATGATATC & $44.9(58.2)$ \\
\hline PaAtg18_C45A_fwd & AACCAGGACTTCTCCGCTGTGTCAGTGGGTTATAG & 77.6 \\
\hline PaAtg18_C59A_fwd & GGTATAAAATCTATAATGCTGAGCCGTTCGGCCAG & 75.2 \\
\hline PaAtg18_C113A_fwd & CGTCAAACCACCATCGCTGAACTGACCTTTCC & 75.9 \\
\hline PaAtg18_C515A_fwd & GAACGTGGCGGTGACGCTGTCCTGCTGCAC & 79.7 \\
\hline paAtg18_C45A_rev & CTATAACCCACTGACACAGCGGAGAAGTCCTGG & 74.9 \\
\hline paAtg18_C59A_rev & CTGGCCGAACGGCTCAGCATTATAGATTTTATACC & 75.2 \\
\hline paAtg18_C65A_rev & GCTTTTCGAATAAGCCTGGCCGAACGGCTC & 75.6 \\
\hline paAtg18_C113A_rev & GGAAAGGTCAGTTCAGCGATGGTGGTTTGACG & 75.9 \\
\hline paAtg18_C515A_rev & GTGCAGCAGGACAGCGTCACCGCCACGTTC & 79.7 \\
\hline paAtg18_C65_fwd2 & TGAGCCGTTCGGCCAGGCTTATTCGAAAAGC & 75.8 \\
\hline paAtg18_S51C_fwd & GTGTCAGTGGGTTATTGCAATGGGTATAAAATC & 74.2 \\
\hline paAtg18_S51C_rev & GATTTTATACCCATTGCAATAACCCACTGACAC & 74.2 \\
\hline paAtg18_S81C_fwd & GTGGAAATGCTGTTCTGCTCATCTCTGCTGGC & 79.1 \\
\hline paAtg18_S81C_rev & GCCAGCAGAGATGAGCAGAACAGCATTTCCAC & 79.1 \\
\hline paAtg18_S157C_fwd & CGATTGAAACCCCGTGCAATCCGAATGGTC & 77.5 \\
\hline paAtg18_S157C_rev & GACCATTCGGATTGCACGGGGTTTCAATCG & 77.5 \\
\hline paAtg18_S459C_fwd & GTGGTGGGTGTTGGTTGTAAAATCTGGGACG & 77.7 \\
\hline paAtg18_S459C_rev & CGTCCCAGATTTTACAACCAACACCCACCAC & 77.7 \\
\hline paAtg18_S448C_fwd & CTGAAAGTTCCTGCTTGCAAAGAAACCAAAACC & 75.4 \\
\hline paAtg18_S448C_rev & GGTTTTGGTTTCTTTGCAAGCAGGAACTTTCAG & 75.4 \\
\hline pAce1_XhoI_Fwd & CGTCTCGAGAGATCCGGCT & 58.7 \\
\hline pAce1_NcoI_Rev & TGCCCATGGGTATATCTCCTTCTTAAAG & $41.2(57.9)$ \\
\hline pDK_XhoI_Fwd & CGTCTCGAGACTAGTTCCGTT & 56 \\
\hline MIE_NdeI_Fwd & ATACATATGAGGCCTCGG & 50.8 \\
\hline MIE_XhoI_Rev & TCTCTCGAGACGCGTTCG & 56.8 \\
\hline pBAD_Rev & CTTCTGCGTTCTGATTTAATCTG & 51.9 \\
\hline pBADM-20(+)_Fwd & CATCATCATCATCATTCTTCTGG & 51.4 \\
\hline pBADM-30(+)_Fwd & ATAGCATGGCCTTTGCAGG & 55.8 \\
\hline pBADM-41(+)_Fwd & TCGTCAGACTGTCGATGAAGCC & 58.8 \\
\hline pBAD-52(+)_Fwd & CATCACCATCACCATCAC & 50.5 \\
\hline pGex_rev & GAGCTGCATGTGTCAGAGG & 55.8 \\
\hline T7_ctrl_Fwd & TCACTATAGGGGAATTGTGAGCGG & 58.3 \\
\hline T7_ctrl_Rev & CTAGTTATTGCTCAGCGGTGGC & 58.1 \\
\hline Lac_ctrl_Fwd & GTATGTTGTGTGGAATTGTGAGCG & 56.6 \\
\hline Lac_ctrl_Rev & TACGAAGTTATCTGCCAGGCAC & 56.9 \\
\hline
\end{tabular}




\subsection{Experimental methods}

\subsubsection{Molecular cloning}

Standard methods were used for molecular cloning. PCR's were done with the Phusion High-Fidelity PCR kit. The reaction mixture for one elongation reaction is given in Table 2.11. The reaction mixture was split in three PCR tubes. The PCR program is given in Table 2.12 Elongation time was adjusted to template length. Furthermore the annealing temperature was chosen according to the melting temperature of the primers (see table 2.10).

Table 2.11: PCR reaction mixture

\begin{tabular}{ll}
\hline Component & Volume $(\mu \mathrm{L})$ in $75 \mu \mathrm{L}$ reaction \\
\hline Template DNA & $5 \mathrm{ng} / 1 \mathrm{~kb}$ of the template \\
$5 \mathrm{x}$ Phusion HF & $15 \mu \mathrm{L}$ \\
Forward primer $(5 \mu \mathrm{M})$ & $6 \mu \mathrm{L}$ \\
Reverse primer $(5 \mu \mathrm{M})$ & $6 \mu \mathrm{L}$ \\
dNTP-mix $(10 \mathrm{mM})$ & $1.5 \mu \mathrm{L}$ \\
Phusion polymerase $(2 \mathrm{U} / \mu \mathrm{L})$ & $0.75 \mu \mathrm{L}$ \\
Deionized water & $\mathrm{Up} \mathrm{to} 75 \mu \mathrm{L}$ of the final volume \\
\hline \hline
\end{tabular}

Table 2.12: PCR program

\begin{tabular}{|c|c|c|}
\hline Step & Time & Temperature \\
\hline Initial denaturation & $30 \mathrm{sec}$ & $98^{\circ} \mathrm{C}$ \\
\hline Denaturation & $5 \mathrm{sec}$ & $98^{\circ} \mathrm{C}$ \\
\hline Annealing & $15 \mathrm{sec}$ & $\begin{array}{l}\text { higher } \mathrm{Tm} \text { of the matching sequence } \\
\text { for the pair of primers }+5^{\circ} \mathrm{C} \text {; touch } \\
\text { down }-0.7^{\circ} / \text { cycle }\end{array}$ \\
\hline Elongation & $15 \mathrm{sec} / \mathrm{kb}$ & $72^{\circ} \mathrm{C}$ \\
\hline \multicolumn{3}{|l|}{ Repeat 14 times } \\
\hline Denaturation & $5 \mathrm{sec}$ & $98^{\circ} \mathrm{C}$ \\
\hline Annealing & $15 \mathrm{sec}$ & $\begin{array}{l}\text { lower Tm of the full length sequence } \\
\text { for the pair of primers }\end{array}$ \\
\hline Elongation & $15 \mathrm{sec} / \mathrm{kb}$ & $72^{\circ} \mathrm{C}$ \\
\hline \multicolumn{3}{|l|}{ Repeat 6 times } \\
\hline Final extension & $3 \mathrm{~min}$ & $72^{\circ} \mathrm{C}$ \\
\hline Storage & & $8^{\circ} \mathrm{C}$ \\
\hline
\end{tabular}


The PCR products were analyzed by DNA electrophoresis in $0.8 \%$ agarose gel run in TAE buffer under 90 $\mathrm{V}$ voltage for 1 hour. Next, PCR products were digested with $\mathrm{DpnI}\left(0.3 \mathrm{U} / \mu \mathrm{L}\right.$, overnight, $\left.37^{\circ} \mathrm{C}\right)$ to remove the methylated template vector. The restriction was stopped by $20 \mathrm{~min}$ incubation at $80^{\circ} \mathrm{C}$. The PCR products were purified using QIAquick PCR Purification Kit (Qiagen). The resulting product together with the destination vectors were digested overnight or 4 hours at $37^{\circ} \mathrm{C}$ with the chosen pair of restriction enzymes (NEB). Optimal digestion conditions were selected according to the recommendations of the manufacturer. Digested inserts were then purified using QIAquick PCR Purification Kit. Digested vectors were run on $0.8 \%$ agarose gel and subsequently extracted using the QIAquick Gel Extraction Kit (Qiagen).

Ligation of inserts with the digested vectors was performed using T4 ligase. Concentration of vector was set to approximately $20 \mathrm{ng}$ and vector to insert molar ratio was $1: 4.0 .5 \mu \mathrm{L}$ of T4 ligase ( $400 \mathrm{U} / \mu \mathrm{L}, \mathrm{NEB})$ and $1.0 \mu \mathrm{L}$ of $10 \mathrm{x}$ T4 buffer (NEB) were added to a total volume of $10 \mu \mathrm{L}$. Ligation was performed for 1 hour at room temperature.

The resulting construct $(4 \mu \mathrm{L})$ was transformed in Escherichia coli XL1-Blue or DH5 $\alpha$ chemocompetent bacteria. Bacteria were incubated with DNA for 30 min incubation on ice. Next, the bacteria DNA mixture underwent a heat shock: $45 \sec 42^{\circ} \mathrm{C}$ in a water bath, $2 \min 4^{\circ} \mathrm{C}$. Subsequently, bacteria were mixed with 900 $\mu \mathrm{L}$ pre-warmed SOC medium and incubated for $1 \mathrm{~h}$ at $37^{\circ} \mathrm{C}$ with shaking. Transformed bacteria were plated on LB-agar plates with supplemented antibiotic.

The cloning of the correct insert was checked by colony PCR. Eight colonies from each plate were transferred to a new LB-agar plate with antibiotics with a sterile tip that subsequently was washed in $10 \mu \mathrm{L}$ of PCR mix. Taq polymerase (Qiagen) and vector specific primers (Table 2.10) were used for the colony PCR. Recipe of the mix for 10 reactions is given in Table 2.13 and the program used in Table 2.14. The resulting DNA was checked for expected size using a $0.8 \%$ agarose gel.

Table 2.13: PCR reaction mixture for colony PCR

\begin{tabular}{ll}
\hline Component & Volume $(\mu \mathrm{L})$ \\
\hline Deionized water & 71 \\
Forward primer & 8 \\
Reverse primer & 8 \\
dNTP $(10 \mathrm{mM})$ & 2 \\
10x Taq buffer (Qiagen) & 10 \\
Taq polymerase (Qiagen) & 1 \\
\hline \hline
\end{tabular}


Table 2.14: PCR program for colony PCR

\begin{tabular}{lll}
\hline Step & Time & Temperature $\left({ }^{\circ} \mathrm{C}\right)$ \\
\hline Initial denaturation & $2 \mathrm{~min}$ & 94 \\
\hline Denaturation & $15 \mathrm{sec}$ & 94 \\
Annealing & $30 \mathrm{sec}$ & $\mathrm{Tm}-5$ \\
Elongation & $15 \mathrm{sec} / \mathrm{kb}$ & 72 \\
Repeat 30 time & & \\
\hline Storage & & 8 \\
\hline \hline
\end{tabular}

In order to isolate plasmids, two colonies that showed the correct insert length, were inoculated into $6 \mathrm{~mL}$ of ZYM505 medium with an appropriate antibiotic and incubated overnight at $37^{\circ} \mathrm{C}$ with shaking. Plasmids were isolated using NucleoSpin Plasmid kit (Macherey-Nagel). Purified plasmids were checked by sequencing (Eurofins, MWG Operon).

For the recombination of a pAce1-N-His vector with pDk-N-StrepII vector to generate a pACEMBL plasmid, Cre recombinase was used. pDk and pAce1-N-His plasmids in amount of $1 \mu \mathrm{g}$ each were mixed together with $2 \mu \mathrm{L}$ of Cre recombinase and $2 \mu \mathrm{L}$ of $10 \mathrm{x}$ Cre buffer in a $20 \mu \mathrm{L}$ reaction mixture. The reaction was incubated for $1 \mathrm{~h}$ at $37^{\circ} \mathrm{C}$. XL1-Blue bacteria were transformed with recombined vector and were plated on the LB-agar medium supplemented with kanamycin and ampicillin. Next day, four of the resulting colonies were inoculated in $6 \mathrm{~mL}$ ZYM505 medium with kanamycin for overnight growth. Plasmids were isolated and checked for correct restriction digest pattern.

Mutagenesis of PaAtg18 was done using the QuickChange Ligthning site-directed mutagenesis kit. The PCR mix contained $5 \mu 1$ 10x reaction buffer, 20 ng DNA template, $1 \mu \mathrm{l}$ dNTP mix, up to $50 \mu 1 \mathrm{H}_{2} \mathrm{O}$ and $1 \mu \mathrm{l}$ PfuUltra HF DNA polymerase $(2.5 \mathrm{U} / \mu \mathrm{l})$. The reaction volume was split in two and the PCR primer solution volume corresponding to $125 \mathrm{ng}$ oligonucleotide of fwd primer was added in the first one and similarly, $125 \mathrm{ng}$ oligonucleotide primer was added in the second one. The PCR reaction was run with the program given in Table 2.15 Afterwards, template DNA was digested by DpnI-treatment. Finally, the PCR product was transformed with E. coli XL1-blueGOLD supercompetent cells.

Table 2.15: PCR program for mutagenesis

\begin{tabular}{lll}
\hline Step & Time & Temperature $\left({ }^{o} \mathrm{C}\right)$ \\
\hline Initial denaturation & $30 \mathrm{sec}$ & 98 \\
\hline Denaturation & $15 \mathrm{sec}$ & 98 \\
Annealing & $30 \mathrm{sec}$ & 55 \\
Extension & $35 \mathrm{sec} / 1 \mathrm{~kb}$ plasmid size & 72 \\
repeat 5 times & & \\
\hline mix the two reactions & & \\
repeat the above program 13 times & & \\
\hline
\end{tabular}




\begin{tabular}{lll}
\hline Step & Time & Temperature $\left({ }^{o} \mathrm{C}\right)$ \\
\hline Final extension & $3 \mathrm{~min}$ & 72 \\
Storage & & 8 \\
\hline \hline
\end{tabular}

\subsubsection{Protein expression and purification}

\subsubsection{Protein expression test}

Proteins of interest were expressed in E. coli strains in which the expression plasmid with the gene of interest was transformed. Pre-culture was prepared by inoculation of a single BL21(DE3) colony into $100 \mathrm{~mL}$ of ZYM505 medium followed by overnight growth at $37^{\circ} \mathrm{C}$ with shaking. Pre-culture $(0.5 \mathrm{~mL})$ was inoculated into $200 \mathrm{~mL}$ of medium with antibiotic. LB, TB, ZYM5052 and MD5052 media were used to test expression at different temperatures conditions. All cultures were grown at $37^{\circ} \mathrm{C}$ until induction time, i.e., their $\mathrm{OD}_{600}$ reached a value close to 0.6. Next, incubation temperature was changed accordingly. LB and TB cultures were induced with $1 \mathrm{mM}$ or $0.1 \mathrm{mM}$ IPTG in the case of $T 7$ promoter or $0.01 \mathrm{mg} / \mathrm{mL}$ arabinose in the case of araBAD promoter. $50 \mathrm{~mL}$ of cultures were harvested at different time points.

Harvested culture was centrifuged $4^{\prime} 000 \mathrm{rpm}, 20 \mathrm{~min}, 4^{\circ} \mathrm{C}$. Pellets were resuspended in $5 \mathrm{~mL}$ of the lysis buffer. Resuspended cells were lysed by sonication ( 3 cycles of $30 \mathrm{sec}$ impulse and $30 \mathrm{sec}$ rest on ice). Bacterial lysate $(3 \mathrm{~mL})$ were collected and centrifuged $13^{\prime} 000 \mathrm{rpm}, 30 \mathrm{~min}, 4^{\circ} \mathrm{C}$. After centrifugation, $2 \mathrm{~mL}$ of supernatant were incubated with equilibrated $\mathrm{Ni}^{2+}$ - or GST-sepharose beads for 2 hours or overnight at $4^{\circ} \mathrm{C}$. Whole cell lysate $(50 \mu \mathrm{L})$, supernatant after centrifugation $(50 \mu \mathrm{L})$ and beads sample were collected for the SDS-PAGE analysis.

\subsubsection{Protein expression and purification}

Expression strains, media, temperatures and durations are summarized in Table 2.16 for all proteins used in this study.

Table 2.16: Expression conditions for proteins used in this study

\begin{tabular}{llll}
\hline Protein & Media & Temperature $\left({ }^{\circ} \mathrm{C}\right)$ & Duration \\
\hline ScAtg18 & TB & 18 & over night \\
ScAtg21 & TB & 18 & over night \\
CtAtg18 & MD5052 & 18 & over night \\
PaAtg18 (and mutants) & ZYM5052 & 25 & over night \\
K1Atg21 & ZYM5052 & 22 & over night \\
ScHsv2 (and mutants) & ZYM5052 & 25 & over night \\
MBP-CtAtg2(1-270) & TB & 25 & over night \\
MBP-CtAtg2(1-279) & TB & 25 & over night \\
AtAtg18a & MD5052 & 18 & $5 \mathrm{~h}$
\end{tabular}




\begin{tabular}{llll}
\hline Protein & Media & Temperature $\left({ }^{\circ} \mathrm{C}\right)$ & Duration \\
\hline AtWRKY33 & MD5052 & 18 & $5 \mathrm{~h}$ \\
\hline \hline
\end{tabular}

Pre-culture of E. coli BL21(DE3) was prepared in $100 \mathrm{~mL}$ ZYM505 medium by inoculating a single colony. After overnight incubation, $10 \mathrm{~mL}$ of pre-culture were inoculated into six $5 \mathrm{~L}$-flasks, in $1 \mathrm{~L}$ of medium each. Expression was done, as optimized during the expression test, as summarized in Table tab:expressionconditions.

After incubation, cultures were harvested and centrifuged $\left(4^{\prime} 000 \mathrm{rpm}, 20 \mathrm{~min}, 4^{\circ} \mathrm{C}\right)$. Pellets were resuspended by shaking in $10 \mathrm{~mL}$ of lysis buffer without lysozyme. Cells were opened using microfluidizer M-110L (Microfluidics Corporation) by pumping them through the system three times. Cell lysates were spun down at $14^{\prime} 000 \mathrm{rpm}, 45 \mathrm{~min}, 4^{\circ} \mathrm{C}$. The supernatant, containaing the soluble protein fraction, was filtered through 0.4 $\mu \mathrm{m}$ filter.

Filtered supernatant was loaded on the specific affinity column as follows: ScHsv2 (and its mutants), ScAtg18 and ScAtg21 were purified using GSTrap, AtWRKY33 using a StrepTrap, while the rest of the proteins were purified using a HisTrap. Purifications were done using Äkta Prime system. The HisTrap purification was a gradient elution. Its program is summarized in Table 2.17 The proteins loaded on the StrepTrap and GSTrap columns were eluted in a step elution as shown in Table 2.18.

Table 2.17: Purification program for HisTrap. $X^{*}$ - injected volume.

\begin{tabular}{lllllll}
\hline Step & Breakpoint $(\mathrm{mL})$ & $\%$ Buffer B & $\begin{array}{l}\text { Flow rate } \\
(\mathrm{mL} / \mathrm{min})\end{array}$ & $\begin{array}{l}\text { Fraction size } \\
(\mathrm{mL})\end{array}$ & Position \\
\hline Equilibration & 0 & 0 & 5 & 0 & Load \\
Sample inject & 50 & 0 & 1 & 7 & Inject \\
Wash & $50+\mathrm{X}^{*}$ & 0 & 1.5 & 7 & Load \\
1st gradient & $150+\mathrm{X}$ & 0 & 1 & 2 & Load \\
2nd gradient & $200+\mathrm{X}$ & 20 & 1 & 2 & Load \\
Elution & $250+\mathrm{X}$ & 100 & 1 & 2 & Load \\
End & $300+\mathrm{X}$ & 100 & 1 & 2 & Load \\
\hline \hline
\end{tabular}

Table 2.18: Purification program for GSTrap and StrepTrap

\begin{tabular}{llllll}
\hline Step & Breakpoint $(\mathrm{mL})$ & $\%$ Buffer B & $\begin{array}{l}\text { Flow rate } \\
(\mathrm{mL} / \mathrm{min})\end{array}$ & $\begin{array}{l}\text { Fraction size } \\
(\mathrm{mL})\end{array}$ & Position \\
\hline Equilibration & 0 & 0 & 5 & 0 & Load \\
Sample inject & 50 & 0 & 1 & 7 & Inject \\
Wash & $50+\mathrm{X}^{*}$ & 0 & 1.5 & 7 & Load \\
Step & $150+\mathrm{X}$ & 0 & 1 & 2 & Load
\end{tabular}




\begin{tabular}{llllll}
\hline Step & Breakpoint $(\mathrm{mL})$ & $\%$ Buffer B & $\begin{array}{l}\text { Flow rate } \\
(\mathrm{mL} / \mathrm{min})\end{array}$ & $\begin{array}{l}\text { Fraction size } \\
(\mathrm{mL})\end{array}$ & Position \\
\hline Elution & $150.1+\mathrm{X}$ & 100 & 1 & 2 & Load \\
End & $200+\mathrm{X}$ & 100 & 1 & 2 & Load \\
\hline \hline
\end{tabular}

After the affinity chromatography, the collected samples were checked by SDS-PAGE. Fractions containing the protein of interest were pooled together and concentrated to the volume of $5 \mathrm{~mL}$. Before loading the protein sample on the gel filtration column, it was filtered through a $0.2 \mu \mathrm{m}$ filter. Gel filtration was performed in order to improve protein purity and remove aggregates. The gel filtration was performed as desribed in Table 2.19 Elution fractions were analyzed by SDS-PAGE. The fractions containing the protein of interest were pooled and concentrated to $10-40 \mathrm{mg} / \mathrm{mL}$.

Table 2.19: Purification program for gel filtration

\begin{tabular}{llllll}
\hline Step & Breakpoint $(\mathrm{mL})$ & $\%$ Buffer B & $\begin{array}{l}\text { Flow rate } \\
(\mathrm{mL} / \mathrm{min})\end{array}$ & $\begin{array}{l}\text { Fraction size } \\
(\mathrm{mL})\end{array}$ & Position \\
\hline Inject & 0 & 0 & 1 & 0 & Inject \\
Void volume & 7 & 0 & 1 & 0 & Load \\
Elution & 40 & 0 & 1 & 2 & Load \\
End & 127 & 0 & 1 & 2 & Load \\
\hline \hline
\end{tabular}

\subsubsection{Biochemical methods}

\subsubsection{SDS-PAGE and Western Blotting}

SDS-PAGE was done in two-phase polyacrylamide gels consisting of a stacking (25\% stacking gel buffer, $61.4 \%$ water, $12.5 \%$ acrylamide, $1 \%$ APS, TEMED) and a resolving gel (25\% resolving gel buffer, $34 \%$ water, $40 \%$ acrylamide, $1 \%$ APS, TEMED). Before gel analysis, protein samples were diluted in 3x SDS loading buffer $(2: 1)$ and incubated for $5 \mathrm{~min}$ at $95^{\circ} \mathrm{C}$ with shaking. Electrophoretic separation was done in the running buffer under voltage $120 \mathrm{~V}$. Voltage was switched to $150 \mathrm{~V}$ when the dye line had reached the stacking gel. Gels were resolved until the running front reached the very bottom of the gel.

After the gel was run, SDS-PAGE gels were stained using a Coomassie Blue reagent (500 mg Coomassie $\mathrm{R}, 500 \mathrm{~mL} \mathrm{H}_{2} \mathrm{O}, 400 \mathrm{ml}$ ethanol, $100 \mathrm{ml}$ acetic acid), heated in microwave for $1 \mathrm{~min}$. Gels were destained in $10 \%$ acetic acid in $\mathrm{dH}_{2} 0$.

For Western Blotting analysis, resolved gels were blotted to nitrocellulose membranes. The semidry transfer was assembled from the anode side to the cathode side as follows: 2 pieces of Whatman filter paper - gel nitrocellulose membrane - 2 pieces of Whatman filter paper. All assembled parts were pre-incubated in transfer 
buffer. Blotting was performed for $90 \mathrm{~min}$ at a constant voltage of $25 \mathrm{~V}$. Next, membranes was blocked with 5\% skim milk powder solution in PBS-T for $2 \mathrm{~h}$ at $4^{\circ} \mathrm{C}$. Membrane was washed in PBS-T buffer 5 times for $5 \mathrm{~min}$ and incubated with primary antibody overnight at $4^{\circ} \mathrm{C}$. Membrane washing was repeated after incubation and the membrane was left to incubate with secondary antibody for $2 \mathrm{~h}$ at $4^{\circ} \mathrm{C}$. Incubation was followed by another washing step. Excess of PBS-T was removed and membrane was washed in $1 \mathrm{~mL}$ of ECL HRP substrate solution. Chemiluminiscence signal was determined with Imageready LAS-1000 CCD camera (Fujifilm).

\subsubsection{Protein stability assay}

Protein stability in different conditions was tested by a thermofluor assay [154]. Protein samples (16 $\mu \mathrm{L}$ of at a concentration of $5 \mu \mathrm{M}$ ) were pipetted into a 96-well plate (Biozym Scientific $\mathrm{GmbH}$ ). Water and buffer controls were set in the last column. Each control was replicated four times. Conditions from pHat or ADDit screens (Emerald Biosystems) were added into corresponding wells $(2 \mu \mathrm{L})$. The protein buffer was added into wells A12:H12 in amount of $2 \mu \mathrm{L}$. Sypro Orange dye was added into each well (2 $\mu \mathrm{L}$ of 1:50 dilution of 10000x stock). 96-well plate was incubated into the CFX96 Real-Time System (C1000 Thermal Cycler, BioRad). The plate was heated from $25^{\circ} \mathrm{C}$ to $95^{\circ} \mathrm{C}$ to monitor protein folding by changes in the fluorescence intensity. The results were analyzed using the DSF Excel macro, version 2.5 (available from: ftp://ftp.sgc.ox.ac.uk/pub/biophysics).

\subsubsection{Circular dichroism spectroscopy}

Measurements were done with a Chirascan Circular Dichroism spectrometer (Applied Photophysics) using a Hellma quartz cuvette with a path length of $0.1 \mathrm{~cm}$. Samples were in a $30 \mathrm{mM}$ sodium phosphate buffer $\mathrm{pH}$ 7 containing $150 \mathrm{mM} \mathrm{NaF}$. Far UV CD spectra of $10 \mu \mathrm{M}$ protein were recorded between 200 and $260 \mathrm{~nm}$ with a step size of $1 \mathrm{~nm}$, a bandwidth of $0.5 \mathrm{~nm}$ and an averaging time of $3 \mathrm{sec}$ at room temperature. Thermal melts were carried out from $30^{\circ} \mathrm{C}$ to $80^{\circ} \mathrm{C}$ at $216 \mathrm{~nm}$ with a heating rate of $0.2^{\circ} \mathrm{C} / \mathrm{min}$ with a temperature step of $0.5^{\circ} \mathrm{C}$. Bandwidth was $0.5 \mathrm{~nm}$ and the averaging time was $3 \mathrm{~s}$.

Data were analyzed with the manufacturer's ProView Software. Melting curves were fitted to a sigmoid shape and melting temperatures were determined as the inflection point, $\mathrm{x} 0$ of the fitting function:

$$
f(x)=A_{b}+\frac{\left(A_{t}-A_{b}\right)}{\left(1+e^{\left(x_{0}-x\right) / w}\right)}
$$

where $w$ is the width of the sigmoidal slope. $A_{t}$, the maximum ellipticity, corresponds to the unfolded protein, while $A_{b}$, minimum ellipticity, corresponds to the fully folded protein.

\subsubsection{Protein-lipid co-flotation assay and liposome preparation}

Flotation assays with small unilamellar vesicles (SUV) were used to check that the PI binding affinity in the PaAtg18 mutants was maintained. For preparation of liposomes, a 1-mg containing lipid mix composed of $73 \%$ (w/w) L-phosphatidylcholine isolated from chicken egg (840051C; Avanti Polar Lipids); 2\% Texas Red-1,2dihexadecanoyl-sn-glycero-3-phosphoethanolamine (DHPE), triethylammonium salt (T1395MP; Invitrogen); 24\% L- phosphatidylethanolamine (brain, porcine; 840022P; Avanti Polar Lipids); and 1\% phosphatidylinositol(3,5)-biphosphate (1,2-dioleoyl-sn-glycero-3-phospho-[1'-myo-inositol-3',5' -biphosphate]), ammonium salt (850154P; 
Avanti Polar Lipids) was pipetted together and dried. Dried lipids were resuspended in with $150 \mu \mathrm{L}$ of HP150 buffer containing 3\% cholate. Cholate was removed by size exclusion chromatography using a self-packed column filled with $0.5 \mathrm{~g}$ of Sephadex G50 (Sigma) resuspended in HP150 buffer. Liposome-containing fractions were pooled and stored at $4^{\circ} \mathrm{C}$.

Protein-lipid co-flotation assays were performed using $5 \mu \mathrm{l}$ of $2 \mu \mathrm{M}$ protein incubated with $45 \mu \mathrm{l}$ of liposomes for 15-30 min on ice in 7 x $20 \mathrm{~mm}$ PC tubes (Beckman). Next, the protein liposome sample was gently mixed with $50 \mu \mathrm{l}$ of $80 \%$ Nycodenz (w/v in HP150 buffer) and overlayed with $50 \mu \mathrm{l}$ of $30 \%$ Nycodenz. The gradient was covered with $30 \mu \mathrm{l}$ HP150 buffer. Samples were spun at $55000 \mathrm{rpm}$ (275000 g) for 1.5 hours at $4{ }^{\circ} \mathrm{C}$ in a Sorvall Discovery M150 SE analytical ultracentrifuge (Thermo Scientific) using a S55-S swinging bucket rotor (Thermo Scientific). Afterwards, six $30 \mu \mathrm{l}$ aliquots were colected from top to bottom and analyzed through western blot detected with a Penta His HRP conjugated antibody.

\subsubsection{Fluorescence determination of PaAtg18 membrane orientation}

PaAtg18 single cysteine mutants at a concentration of $1 \mathrm{mg} / \mathrm{mL}$ in HP150 buffer were incubated with IANBD (Invitrogen) dissolved in DMSO to a concentration of $10 \mathrm{mM}$. For the protein, $2 \mathrm{~mL}$ were used and mixed with $30 \mu \mathrm{L}$ of NBD stock solution followed by a $2 \mathrm{~h}$ rotation at room temperature. The excess NBD was removed using a PD10 desalting column. Protein fractions of $500 \mu \mathrm{L}$ were collected and their $280 \mathrm{~nm}$ absorbance was measured using the Nanodrop.

Large unilammelar vesicles (LUVs) were generated by mixing chloroform solutions of the different lipids as follows: a 1-mg containing lipid mix composed of $72 \%(\mathrm{w} / \mathrm{w})$ DOPC, $26 \%(\mathrm{w} / \mathrm{w})$ DOPE and 2\% (w/w) $\mathrm{PI}(3,5) \mathrm{P}_{2}$. Lipids were dried from the organic solvent under a stream of oxygen-free nitrogen, and then the last traces of organic solvent were removed under vacuum for at least $4 \mathrm{~h}$. Dried phospholipids were resuspended in the corresponding buffers by vigorous vortexing and then large unilamellar phospholipid vesicles of approximatively $50 \mathrm{~nm}$ diameter were prepared by extruding (21 times) rehydrated phospholipid suspensions through a 0.1 and 0.05 ţm polycarbonate membranes (Millipore Inc., Bedford, MA, USA) after 5 freeze-thaw cycles,

NBD fluorescence emission (515-620 nm) experiments were performed on a Fluoromax-2 spectrophotometer with a 5-nm slit width and 478-nm excitation at $37^{\circ} \mathrm{C}$ using $0.3 \mu \mathrm{M}$ PaAtg 18 and $0.1 \mathrm{mM}$ LUVs.

\subsubsection{Isothermal titration calorimetry}

ITC measurements was used to measure PROPPIN binding to liposomes using the protocol previously optimized in our laboratory [155]. For preparation of liposomes, a 1-mg containing lipid mix composed of $72 \%$ (w/w) L-phosphatidylcholine isolated from chicken egg (840051C; Avanti Polar Lipids); $2 \%$ Texas Red-1,2dihexadecanoyl-sn-glycero-3-phosphoethanolamine (DHPE), triethylammonium salt (T1395MP; Invitrogen); 24\% L- phosphatidylethanolamine (brain, porcine; 840022P; Avanti Polar Lipids); and 2\% phosphatidylinositol3-phosphate (1,2-dioleoyl-sn-glycero-3-phospho-[1'-myo-inositol-3'-phosphate]), ammonium salt (850150P; Avanti Polar Lipids) was pipetted together and dried. Dried lipids were resuspended in with $150 \mu \mathrm{L}$ of HP150 (150 mM KCl, 20 mM HEPES, pH 7.4) buffer containing 3\% cholate. Cholate was removed by size exclusion chromatography using a self-packed column filled with $0.5 \mathrm{~g}$ of Sephadex G50 (Sigma) resuspended in HP150 buffer. Liposome-containing fractions were pooled and stored at $4^{\circ} \mathrm{C}$. 
I determined the phosphate content of the prepared liposomes using the phosphomolybdate method [156] to calculate the phosphoinositide concentration. For the calculations, I assumed that all phospholipids were equally well incorporated into the liposomes and that $60 \%$ of the total phosphoinositides are accessible on the surface of the liposomes [155].

For isothermal titration calorimetry (ITC) measurements, proteins were dialyzed into HP150 buffer. ITC measurements were done with a VP ITC MicroCalorimeter (MicroCal) at $25^{\circ} \mathrm{C}$. Protein was titrated into liposomes. Titrations were usually carried out with 9 -fold $4 \mu \mathrm{L}$ injections, followed by a 17 -fold $15 \mu \mathrm{L}$ injections. The first injection was $2 \mu \mathrm{L}$ and was always discarded for the data analysis. Protein concentrations were determined using UV absorbance at $280 \mathrm{~nm}$. The protein concentration range used for measurements was 35-80 $\mu \mathrm{M}$ for the wild type proteins, while for the ScHsv2 mutants a range of 85-130 $\mu \mathrm{M}$. The calculated accessible phosphoinositide concentrations were in the range of 4.5-10 $\mu \mathrm{M}$. Data were fitted with a single-site binding model using the MicroCal Origin 7.0 software. The final values given for the thermodynamic parameters are given as averages of several measurements \pm the standard error of measurement (SE).

\subsubsection{Crystallization and structure determination}

\subsubsection{Crystallization screen setup}

Initial crystallization screening experiments were set up in 96-well sitting drop plates (MRC, Hampton Research). In each well, two drops of different protein concentrations were pipetted using the Cartesian Microsys (Cartesian Dispensing Systems) robot. The robot dispensed $100 \mathrm{~nL}$ drops of protein per well and then added $100 \mathrm{~nL}$ screening buffer (reservoir solution). After the robot finished setting drops, the plate was covered with a transparent sealing tape, in order to avoid that the drops dry out. Crystal plates were stored at $20^{\circ} \mathrm{C}$ in an automated Formulatrix crystallization imager which imaged the plate on a pre-set schedule. Images were checked with the Rockmaker main Application software (Formulatrix). The following crystallization screens were used: SaltRx (Hampton Research), $\mathrm{AmSO}_{4}$, Anions, Cations, Classics I+II, Compas, JCSG+, PACT, PEGI+II, pHclearI+II, ProComplex (Qiagen) and Wiz1+2, Wiz3+4 screens (Emerald Biosystems).

In the case of additives or silver bullet screens used for the 96-well format, $100 \mathrm{~nL}$ protein and the $100 \mathrm{~nL}$ reservoir is followed by $50 \mathrm{~nL}$ of the additive/silver bullet screen (100:100:50 $\mathrm{nL}$ program).

After the initial crystallization screens led to crystals, the crystallization condition was further optimized in 24-well Linbro hanging drop pre-greased plates (Jena Bioscience). For initial trials, screening buffers were self made, and if the crystals could not be reproduced, commercial solutions were purchased. For each well $1 \mathrm{~mL}$ of reservoir solution was prepared. $2 \mu \mathrm{L}$ protein and $2 \mu \mathrm{L}$ reservoir buffer were pipetted together on a siliconized cover slip (22 mm diameter). Afterwards the slide was flipped upside down onto the greased well to seal it air tight. When additives were used, $1.5 \mu \mathrm{L}$ protein and $1.5 \mu \mathrm{L}$ reservoir buffer were pipetted together on a siliconized cover slip and $0.3 \mu \mathrm{L}$ additive was added.

Streak seeding of crystals was used for crystal optimization in 24-well format. For this, a crystal was crushed with an acupuncture needle or glass rod. Next, the needle was streaked through a newly set up drop.

One optimization strategy involved (random) microseeding. Microseeds are prepared by adding $2 \mu \mathrm{L}$ reservoir solution on top of a crystal condition on a 24 -well plate and using a glass rod for finely crushing the crystals. This is washed off the glass cover with $45 \mu \mathrm{L}$ mother liquor followed by another $45 \mu \mathrm{L}$ mother liquor. The resulting solution is moved to a $1.5 \mathrm{~mL}$ microreaction tube and a teflon bead is added. This is vortexed 
three times for $30 \mathrm{sec}$ separated by 2 min ice cooling. The resulting microseeds are used as seeds in both 96well format and 24-well format at different dilutions. For random microseeding, the commercial screens are used with an addition of microseeding solution. The 100:100:50 $\mathrm{nL}$ program is used for this purpose.

Another optimization method are 96-well plate refinement grid or random screens. The screens were planed using the RockMaker software while the Tecan robot was used to pipette the screens from stock solutions into $2 \mathrm{ml}$ 96-well master blocks. From these 96-well MRC plates for crystallization were pipetted.

Finally, the PaAtg18 crystals were obtained through in situ proteolysis. This method is based on small amounts of protease cleaving flexible protein regions to improve crystal packing contacts. For in situ proteolysis crystallization, PaAtg18 was mixed with Proteinase K, subtilisin or trypsin in a 1:2000 molar ratio and kept on ice until setting of the crystallization plates.

\subsubsection{Flash cooling of crystals}

In order to prevent ice formation on the crystal, cryoprectants were used for flash cooling. Cryoprotactants such as ethylene glycol, glycerol, xylitol, sucrose, PEG 400, PEG 8000 were prepared, unless specified otherwise, with the same solution as the reservoir solution (mother liquor) in which the crystals grew. After crystals were fished, they were put in fresh mother liquor, then transferred into a mixture of 1:1 mother liquor and cryoprotectant and, finally to cryoprotectant. In the case of PFPE, crystals were directly moved through the PFPE drop. Finally, crystals were flash frozen in liquid nitrogen.

\subsubsection{Computational methods for structure determination}

X-ray diffraction data were collected at $100 \mathrm{~K}$ at beamline X10SA (Swiss Light Source, Paul Scherrer Institute, Villigen, Switzerland). Data were processed and scaled with the XDS software package [157], which contains three programs: XDS, XSCALE and XDSCONV. XDS performs eight subroutines:

- XYCORR, INIT and COLSPOT determine and correct the spots versus the background of the dataset

- IDXREF indexes the spots

- INTEGRATE integrates the spots

- CORRECT refines unit cell dimensions and determined the space group

For XDS, the collection data parameters have to be offered as an input. Subsequently, XSCALE is used for scaling and merging of data, while XDSCONV converts the output to a format needed for the next step. Processed data was converted to .mtz file format. For PaAtg18, it was not possible to perform molecular replacement with KlHsv2, hence, phasing needed to be performed by single anomalous diffraction (SAD). Selenium incorporated in the protein as selenomethionine was used as an anomalous scatterer. The AutoSol Phenix [158] program was used for phasing. After determination of the Se positions, PHASER, another Phenix program, was used for refining and density modification of the initial map. Successive manual model building was done with Coot [159] while refinement was performed with Phenix. Structure validation was done with the tools available in Coot and Phenix [158]. 


\subsection{Theoretical methods}

\subsubsection{Introduction to molecular dynamics}

Molecular dynamics (MD) is the most popular method of simulating macromolecules in motion. MD tries to describe time evolution of molecular systems based on Newton's equations of motion such as equation 2.2 . However, in doing so, it ignores the forces acting on electrons and considers each atom as represented only by the position of the nucleus. This method is a good approximation of systems with many atoms and the result is obtained in a small computer time. However, force field methods cannot calculate properties that depend on the electronic distribution of the molecule. For this level of calculations, one would need quantum mechanical molecular mechanics calculations.

At each time step, the acceleration, $\vec{a}_{i}$ of the particle $\mathrm{i}$ is calculated using the Newton law shown in equation 2.2

$$
\vec{F}_{i}=m_{i} \vec{a}_{i}
$$

In equation 2.2, $\vec{F}_{i}$ is the force acting on particle $\mathrm{i}$ and $m_{i}$ is the mass of the particle. The positions, $\vec{x}_{i}$ of the particle are obtained through numerical integration from equation 2.3 .

$$
\vec{a}_{i}=\frac{d^{2} \vec{x}_{i}}{d t^{2}}
$$

The force $\vec{F}_{i}$ acting on particle $\mathrm{i}$ is computed, using equation 2.4, at each time step using the potential energy, $V$.

$$
\vec{F}_{i}=-\nabla V
$$

The potential energy, $V$ is calculated as a sum of terms describing interactions between atoms such as covalent bond lengths, angles, torsion angles (proper dihedrals), improper dihedrals (to maintain tetrahedral or planar geometries such as stereochemistry or benzene rings), and a number of non-bonded terms. The bonded interactions are graphically depicted in Figure 2.1. In general, the non-bonded terms include a Lennard-Jones term and an electrostatic (Coulomb) contribution, and in some cases an explicit hydrogen-bonding term. The calculation of the potential $V$ due to bonded interactions is given in equation 2.5

$$
V(\vec{r})=\sum_{n=1}^{N_{b}} V^{(b)}\left(b_{n}, k_{n}^{(b)}, b_{n}^{0}\right)+\sum_{n=1}^{N_{\theta}} V^{(\theta)}\left(\theta_{n}, k_{n}^{(\theta)}, \theta_{n}^{0}\right)+\sum_{n=1}^{N_{\Phi}} V^{(\Phi)}\left(\Phi_{n}, k_{n}^{(\Phi)}, \Phi_{n}^{0}\right)+\sum_{n=1}^{N_{\Psi}} V^{(\Psi)}\left(\Psi_{n}, k_{n}^{(\Psi)}, \Psi_{n}^{0}\right)
$$

The bond stretching potential energy is given by the sum of functions $V^{(b)}$ over all $N^{(b)}$ bonds in the system. $V^{(b)}$ is the function describing the potential energy associated with the stretching of a single bond. In general, this function is a harmonic potential but can be also represented through more complex functions. Bond length $b_{n}$ describes the $\mathrm{n}^{\text {th }}$ bond. The quantities $k_{n}^{(b)}$ and $b_{n}^{0}$ are force-field parameters, force constant and reference bond length, respectively, characteristic for specific bond $\mathrm{n}$. 
bond stretching
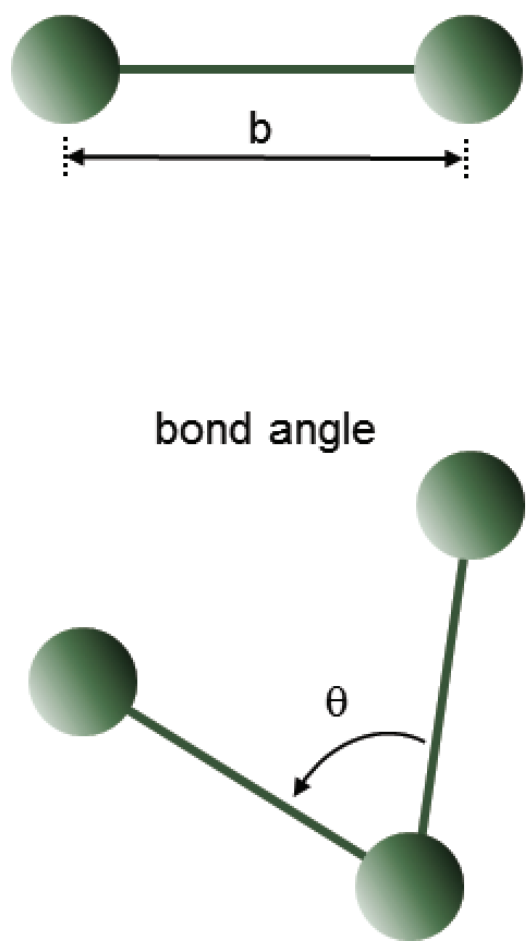

\section{proper dihedral}

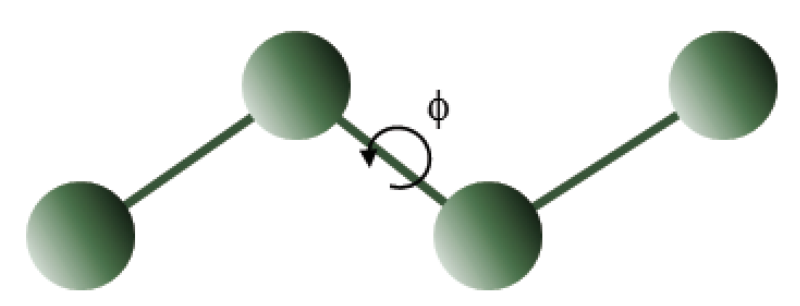

improper dihedral

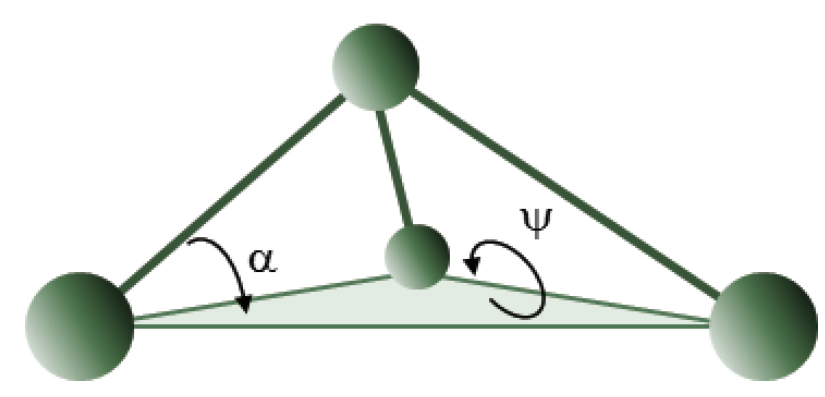

Figure 2.1: Introduction to MD simulations.

MD is based on a simple model of interactions between atoms described by bond stretching, bond angles, torsion angles (dihedrals) and improper dihedrals (to maintain tetrahedral or planar geometries).

Similarly, the bond-angle bending interactions potential energy is given by the sum of functions $V^{(\theta)}$ over all $N^{(\theta)}$ covalent bond-angles present in the system. $V^{(\theta)}$ is the function describing the potential energy associated with the bending of a single bond-angle. In general, this function is a harmonic potential. Bond angle $\theta_{n}$ describes the $\mathrm{n}^{\text {th }}$ bond angle. The quantities $k_{n}^{(\theta)}$ and $\theta_{n}^{0}$ are force-field parameters, force constant and reference angle, respectively, characteristic for specific bond angle $n$.

In a similar manner, the proper dihedral-angle torsion contribution to the potential energy is given by the sum of functions $V^{(\Phi)}$ over all $N^{(\Phi)}$ proper dihedrals present in the system. $V^{(\Phi)}$ is the function describing the potential energy associated with the torsion of a single proper dihedral-angle. Dihedral angle $\Phi_{n}$ describes the $\mathrm{n}^{\text {th }}$ proper dihedral-angle. The quantities $k_{n}^{(\Phi)}$ and $\Phi_{n}^{0}$ are force-field parameters, force constant and reference dihedral, respectively, characteristic for specific dihedral angle $\mathrm{n}$.

The improper dihedral-angle bending energy term is given by the sum of functions $V^{(\Psi)}$ over all $N^{(\Psi)}$ improper dihedral-angles present in the system. $V^{(\Psi)}$ is the function describing the potential energy associated with the bending of a single improper dihedral-angle. Improper dihedral-angle $\Psi_{n}$ describes the $\mathrm{n}^{\text {th }}$ improper dihedral-angle. The quantities $k_{n}^{(\Psi)}$ and $\Psi_{n}^{0}$ are force-field parameters, force constant and reference dihedralangle, respectively, characteristic for specific improper dihedral-angle $n$.

GROMACS (GROningen MAchine for Chemical Simulations) [160, 161] is a molecular dynamics simulation package considered to be the fastest program for molecular simulations to date. The GROMACS project was originally started to construct a dedicated parallel computer system for molecular simulations, based on a ring architecture. However, from its start, GROMACS was continuously improved and developed. 


\subsubsection{Trajectory analysis}

\subsubsection{Root mean square deviation from a reference structure}

The root mean square deviation (RMSD) is defined as the deformation of the macromolecular structure with respect to a reference structure. The average value of these deviations is calculated at each time frame of the MD trajectory using equation 2.6. RMSD is calculated over all the atoms in the structure taking into consideration thei mass, $m_{i}$, their configuration at time $t, r_{i}(t)$ and their reference configuration, $r_{i}^{r e f}$. The RMSD is normalized to the total mass of the system, $M$.

$$
R M S D(t)=\sqrt{\frac{1}{M} \sum_{i} m_{i}\left[r_{i}(t)-r_{i}^{r e f}\right]^{2}}
$$

In general, in a MD simulation, in the first $5 \mathrm{~ns}$ it will be observed an increase in the RMSD value, after which an equilibration with small fluctuations around an average values will occur. As a rule of thumb, a structure can reach equilibrium and is considered stable under 2-3 $\AA$. RMSD values above 3-4 $\AA$ show distortion such as unfolding or conformational changes. However, this is not always true, since a small rotation, for example the rotation of one subunit of a protein relative to another or a hinge motion, can lead to large change in RMSD, yet very little overall change in the structure. In order to distinguish between these possibilities, other analysis methods should be done in parallel.

\subsubsection{Root mean square fluctuations from a reference structure}

The root mean square fluctuation (RMSF) of each residue, $i$ is defined as a measure of the dynamics of each atom or residue in the protein structure. It is calculated using equation 2.7. It is an average over all the considered frames, $N_{\text {frames }}$, and takes into consideration the position of the respective residue, $i$ with respect to its average position, $<r_{i}>$ within the simulation time.

$$
\operatorname{RMSF}(i)=\sqrt{\frac{1}{N_{\text {frames }}} \sum_{i}\left[r_{i}(t)-<r_{i}>\right]^{2}}
$$

As a general feature of the $\mathrm{C} \alpha \mathrm{RMSF}$ profile, there is a correspondence between the larger fluctuations and the loop regions of the structure. This is explained by the fact that $\alpha$-helices and $\beta$-sheets show a much lower fluctuation due to the internal hydrogen bonds.

\subsubsection{MD simulations for the ligand docking and ligand docking studies with KIHsv2}

The GROMACS 4.5.4 simulation package [160, 161] was used for all simulations, with a united atom GROMOS96 43a2 [162] force field and the SPC [163] water model. Periodic boundary conditions were applied to the systems. Long-range electrostatics were calculated using the PME method [164, 165] with a real-space cutoff of $1 \mathrm{~nm}$. For the van der Waals interactions, a cutoff of $1 \mathrm{~nm}$ was used. The simulations were performed at $300 \mathrm{~K}$, using the V-rescale, modified Berendsen thermostat [166] with a coupling constant of $0.1 \mathrm{ps}$. A constant pressure of 1 bar was maintained using a Parrinello-Rahman bariostat [167] in an isotropic coupling type with a coupling constant of $2 \mathrm{ps}$ and a compressibility of $4.5 \times 10^{5} \mathrm{bar}^{-1}$. The integrator for the simulations was leap- 


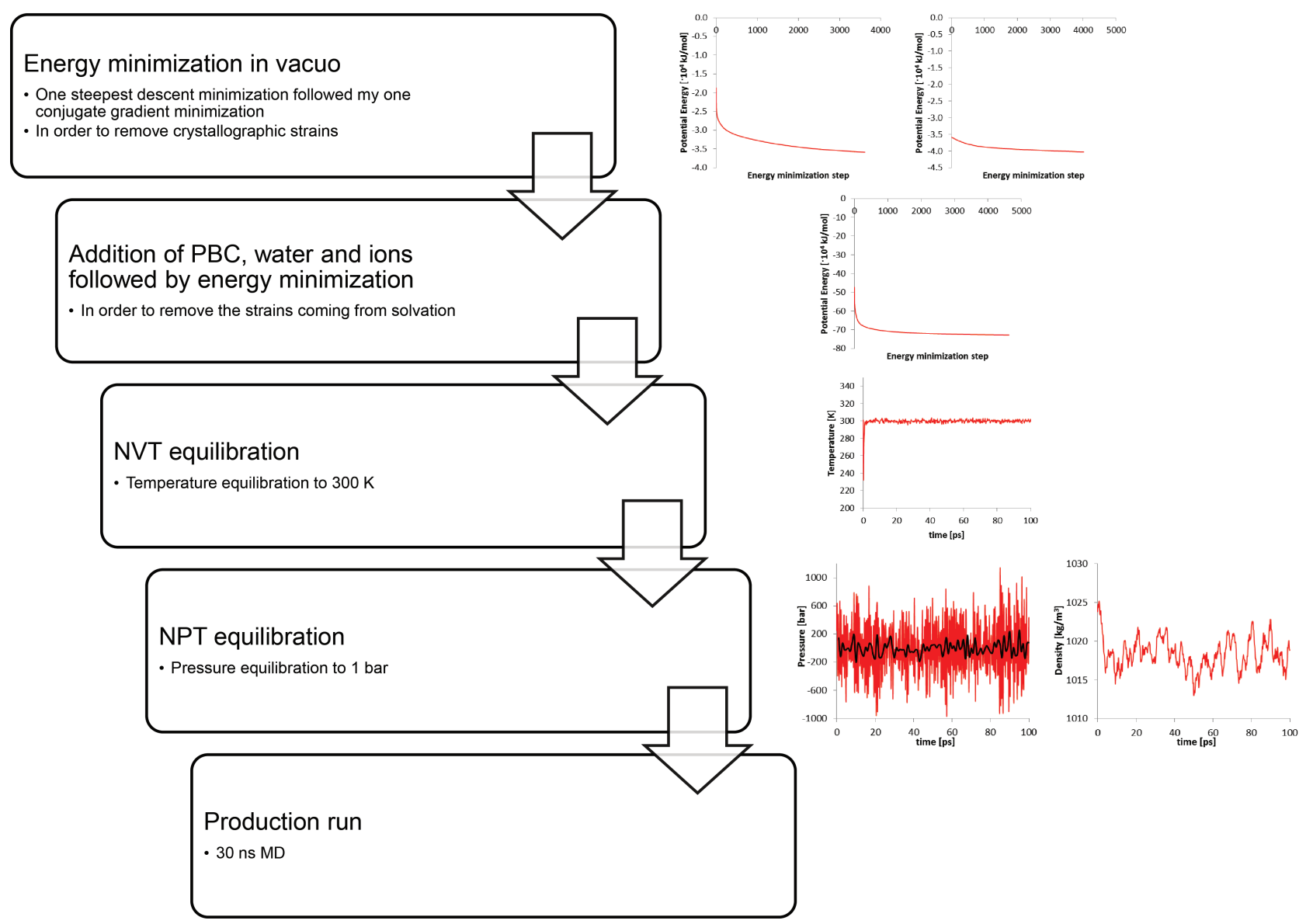

Figure 2.2: MD simulation methodology schematic work flow.

frog integrator with a step of 2 fs. The LINCS method [168] was used to constrain bond lengths. Coordinates were saved every 2 ps for analysis. Analysis of all simulations was performed using the GROMACS package.

The 3.35 A resolution KlHsv2 crystal structure (pdb accession number 4AV9 [1]) was used. This structure contains residues 270-274, part of the loop connecting strands C and D of blade 6. Side chains, which are disordered in the crystal structure, were added to the model with conformations that do not cause steric clashes with Coot [159].

A schematic work flow for the MD simulation procedure is represented in Figure 2.2. For the MD simulation of the apo-protein, energy minimization was done in vacuo, first using steepest descent followed by a conjugate gradient energy minimization. This was done in order to obtain the starting structure. In all the simulations, the protein/ligand or apo-protein were solvated and charge neutralized. The system was then energy-minimized and then two position restrained simulations of 100 ps each were performed for temperature (canonical ensemble) and pressure (isothermic-isobaric ensemble) equilibration. Finally, the systems were simulated for $30 \mathrm{~ns}$.

\subsubsection{Ligand docking studies in KlHsv2}

A schematic work flow for the docking methodology is presented in Figure 2.3. In short, Autodock 4.2 [169] was used for the docking studies. The ligands were allowed torsional flexibility while the macromolecule 
was kept rigid. In order to sample the flexibility of the macromolecule, the ligands were docked in 7 snapshots taken each $5 \mathrm{~ns}$ from a $30 \mathrm{~ns}$ MD simulation of KlHsv2. Grid boxes were centered in the two binding sites (Figure 2.3). The default grid spacing of 0.375 was used. Lamarckian Genetic Algorithm [170] with 2500000 evaluations and 100 runs was used for docking. The schematic diagrams of protein-ligand interactions were visualized with LIGPLOT v.4.5.3 [171].

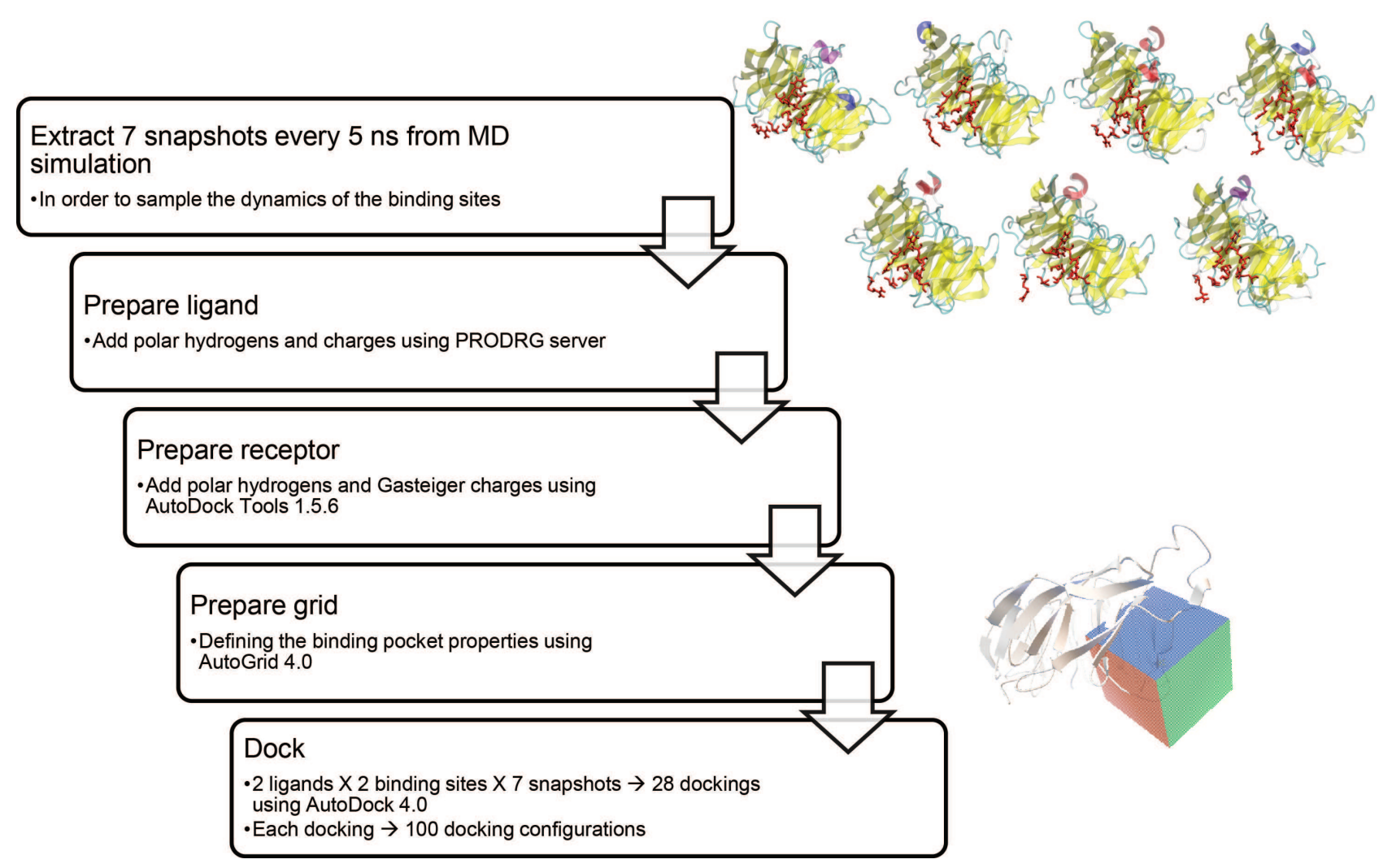

Figure 2.3: Ligand docking methodology schematic work flow.

\subsubsection{PI3P and PI(3,5) $P_{2}$ structures}

The topology of PI3P and PI(3,5) $\mathrm{P}_{2}$ for GROMACS and docking studies was obtained using PRODRG server [172], yielding to a total charge of -3 and -5 , respectively. The atomic partial charges for PI3P were determined by partitioning the molecule in known charge groups for which values are found in literature [162, 173, 174] (Figure 2.4). Using this charge parametrization scheme, the partial charges for all the other six forms of phosphoinositides $\left(\mathrm{PI}(3,5) \mathrm{P}_{2}, \mathrm{PI}(3,4,5) \mathrm{P}_{3}, \mathrm{PI}(3,4) \mathrm{P}_{2}, \mathrm{PI} 4 \mathrm{P}, \mathrm{PI}(4,5) \mathrm{P}_{2}, \mathrm{PI} 5 \mathrm{P}\right)$ can be obtained by exchanging a hydroxyl charge group $(\mathrm{CH}-\mathrm{OH})$ by a phosphate charge group $\left(\mathrm{CH}-\mathrm{O}-\mathrm{PO}_{3}^{2-}\right)$.

\subsubsection{Protein preparation for coarse-grained molecular dynamics simulations of KIHsv2}

The 3.35 Å resolution KlHsv2 crystal structure (pdb accession number 4AV9 [1]) was used. For the coarsegrained MD simulations, the Martini-Elastic protein model [175] was used. In this representation, every four atoms are grouped in a bead. For proteins this implies that each amino acid is represented by one backbone bead and side chain particle(s) depending on the residue type. The structure of the extended strands was stabilized 


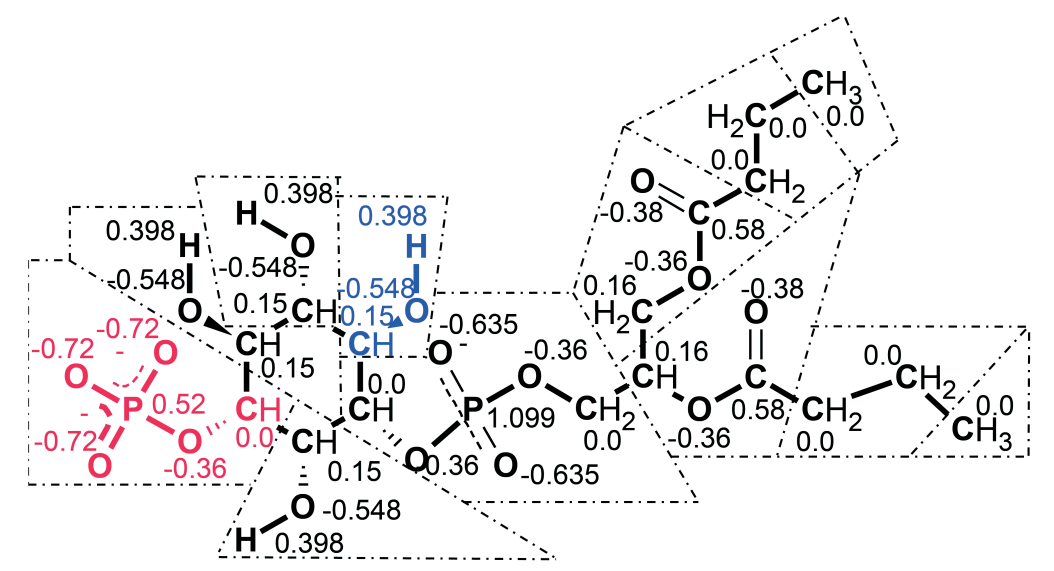

Figure 2.4: Atomic partial charge parametrization of PI3P for GROMOS96 force field.

GROMOS96 building block definitions include charge groups (indicated by dashed boxes) and partial charges (indicated by real numbers). Using this charge parametrization scheme, the partial charges for all the other six forms of phosphoinositides $\left(\mathrm{PI}(3,5) \mathrm{P}_{2}\right.$, $\mathrm{PI}(3,4,5) \mathrm{P}_{3}, \mathrm{PI}(3,4) \mathrm{P}_{2}$, PI4P, $\mathrm{PI}(4,5) \mathrm{P}_{2}$, PI5P) can be obtained by exchanging a hydroxyl charge group, $\mathrm{CH}-\mathrm{OH}$ (blue) by a phosphate charge group, $\mathrm{CH}-\mathrm{O}-\mathrm{PO}_{3}^{2-}$ (red).

by elastic bonds defined by a force constant of $1000 \mathrm{~kJ} \mathrm{~mol}^{-1} \mathrm{~nm}^{-2}$ between all backbone particles that were within $0.7 \mathrm{~nm}$ of each other. The backbones root mean square deviation of the proteins from the initial structure did not exceed $5 \AA$ in any of the simulations performed. This was comparable to an atomistic simulation of the protein in a water box.

\subsubsection{Coarse-grained MD simulations of KIHsv2}

For all coarse-grained MDs the Martini 2.1 force field [175] was used. The time step was 20 fs and the coordinates were saved every 20 ps for subsequent analysis. Periodic boundary conditions were employed. The temperature and pressure were kept constant at the values of $323 \mathrm{~K}$ and 1 bar using the Berendsen ([176]) temperature and pressure coupling algorithms with a time constant of $1 \mathrm{ps}$ for the temperature coupling, while the pressure coupling time constant was 5 ps. Semiisotropic pressure coupling was used with the same compressibility $\left(4.5 \times 10^{5} \mathrm{bar}^{-1}\right)$ in all directions. Lennard-Jones interactions were shifted to zero between 9 and 12 $\AA$ and electrostatics were shifted to zero between 0 to $12 \AA$, with a relative dielectric constant of 20 . Central of mass motion removal was done for linear translational movements of each of the three groups containing the protein, DPPC (dipalmitoyl phosphatidyl choline), and solvent grouped to the ions used for the system charge neutralization.

\subsubsection{DPPC bilayer formation around loop 6CD of KlHsv2}

The GROMACS 4.5.4 simulation package [160, 161] was used for the coarse-grained membrane selfassembly around the loop 6CD of KlHsv2. In this protocol [177, 178], the protein is positioned in the center of a box and lipid molecules are randomly positioned in a cubic box, after which a steepest descent energy minimization is performed. In the present study, the starting dimensions of the box were $10 \mathrm{X} 10 \mathrm{X} 10 \mathrm{~nm}$ and 256 dipalmitoylphosphatidylcholine (DPPC) lipid molecules were used. Water and chloride ions were added to fill the box (3263 molecules and 2 chloride ions) and another steepest descent energy minimization was performed prior to the $100 \mathrm{~ns}$ simulation. 


\subsubsection{Coarse-grained MD simulation of the membrane-KIHsv2 system}

The GROMACS 4.6.5 simulation package [160, 161] was used for a $1 \mu$ s long MD simulation of the assembled bilayer-protein system in a triclinic box of dimensions $9.0556 \times 9.0556 \times 12 \mathrm{~nm}$ with all angles of $90^{\circ}$. The final configuration of the DPPC bilayer formation around loop 6CD of KlHsv2 was stripped of water and energy minimized in vacuo using steepest descent algorithm. Water and chloride ions were added to fill the box and charge neutralize the system. This was followed by a steepest descent energy minimization. Three position-restrained equilibration simulations were performed. The first one was to reach the temperature of $323 \mathrm{~K}$ (canonical ensemble). The length was $100 \mathrm{ps}$, with a time step of $1 \mathrm{fs}$ and position restraints on the protein. Next the pressure of 1 bar was reached through a isothermic isobaric ensemble equilibration of $500 \mathrm{ps,}$ with a time step of $1 \mathrm{fs}$ and position restraints on the protein. Next, the position restraints on the side chains on the protein were removed, and a $1 \mathrm{~ns}$ isobaric ensemble equilibration was performed with a time step of $20 \mathrm{fs}$. Next, a production run was done for $1 \mu \mathrm{s}$.

\subsubsection{Atomistic MD simulations of the KIHsv2-membrane system}

The last frame of the coarse-grained MD simulation of the membrane-protein system was used in order to produce an atomistic level coordinate file of the membrane-protein system using the Sugar-Pie server [179].

Starting from these set of coordinates, the MD simulations were performed using GROMACS 4.6.5 simulation package [160, 161] together with GROMOS96 53A6 force field [180] modified for Berger lipids [181] and SPC water [163]. The histidines of the binding sites, H178 and H249 were protonated after which the system was charge neutralized using five chloride ions. The system was then energy-minimized, and two position-restrained simulations of $100 \mathrm{ps}$ each were performed for temperature (canonical ensemble) and pressure (isothermic isobaric ensemble) equilibration. A production run was done for $100 \mathrm{~ns}$.

Periodic boundary conditions were applied to the system. Electrostatics were calculated using the particle mesh Ewald (PME) method [164, 165] with a real-space cutoff of $12 \AA$. For the van der Waals interactions, a cutoff of $12 \AA$ was used. The simulation was performed at $323 \mathrm{~K}$ using the Nose-Hoover thermostat [182] with a coupling constant of $0.5 \mathrm{ps}$. A constant pressure of 1 bar was maintained using a Parrinello-Rahman bariostat [167] in a semiisotropic coupling type with a coupling constant of 2 ps and a compressibility of $4.5 \times 10^{5} \mathrm{bar}^{-1}$. For the protein system, an isotropic pressure coupling was used. The integrator for the simulation was leap-frog integrator with a step of 2 fs. The LINCS method [168] was used to constrain bond lengths. Central of mass motion removal was done for linear translational movements of each of the three groups containing the protein, DPPC, and solvent grouped to the ions used for the system charge neutralization. For the visualization of the systems, VMD [183] and PyMOL [184] were used.

\subsubsection{Homology modeling}

The steps in the homology modeling protocol are schematically summarized in Figure 2.5. The protein sequences of KlAtg21 and ScHsv2 were each aligned with the sequence of KlHsv2 using T-Coffee server [185]. Behind loop 6CD region, the alignment was manually adjusted. The pdb 4av9 structure of KlHsv2 was used [1] as template. Modeller v9.9 [186, 187] was used to generate 20 initial models. The models were compared using the DOPE score function of Modeller or PROCHECK [188]. The loops in the best model were 


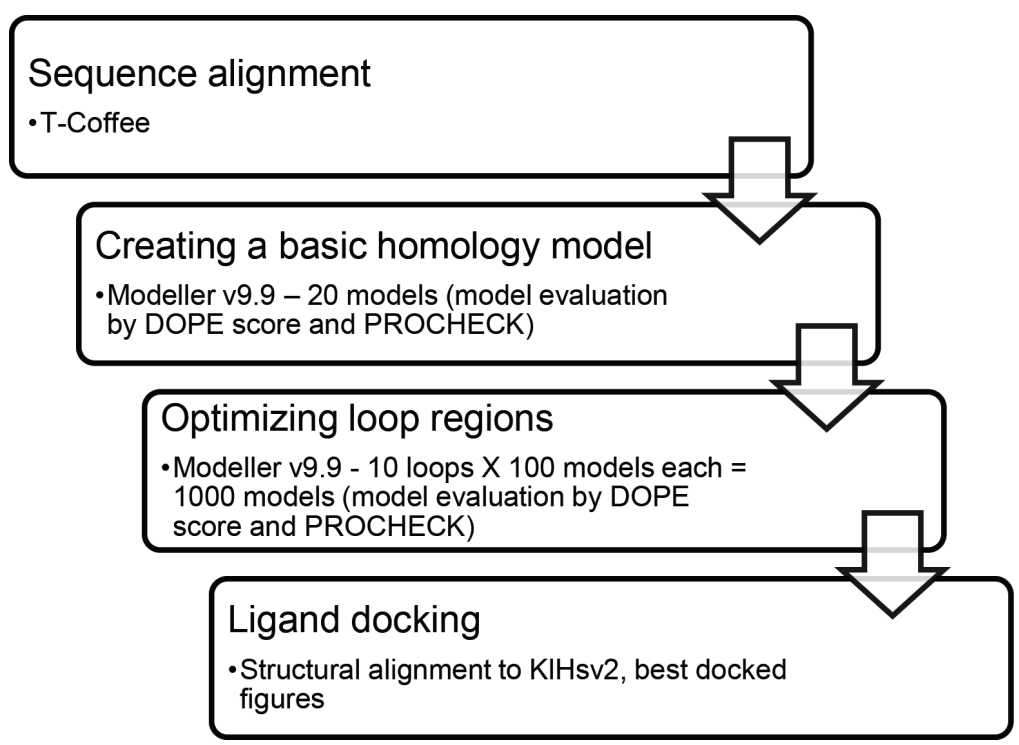

Figure 2.5: Homology modeling methodology schematic work flow.

refined in successive cycles. After each cycle DOPE and PROCHECK evaluations were performed. Finally, the PIs were docked in the active sites of the resulting structures by structural alignment in Pymol [184] with the ligand docked structures of KlHsv2. 


\section{Computational studies of PROPPINs membrane binding}

\subsection{Specific membrane binding of Hsv2}

PROPPINs specifically bind PI3P and PI(3,5) $\mathrm{P}_{2}$. The FRRG motif is highly conserved among PROPPINS of different species and it is essential for phosphoinositide recognition [94]. In this chapter, the details of the recognition by PROPPINs of PI3P and PI $(3,5) \mathrm{P}_{2}$ will be studied using computational methods such as ligand docking and homology modeling. Special attention will be given to K. lactis Hsv2 (KlHsv2) because its crystal structure was determined in our lab [1]].

In our KlHsv2 crystal structure [1], the two arginines of the FRRG motif point towards opposite directions. In the vicinity of these arginines, there are two bound sulfates. These sulfates form salt bridges to highly conserved residues in KlHsv2 (Figure 3.1).

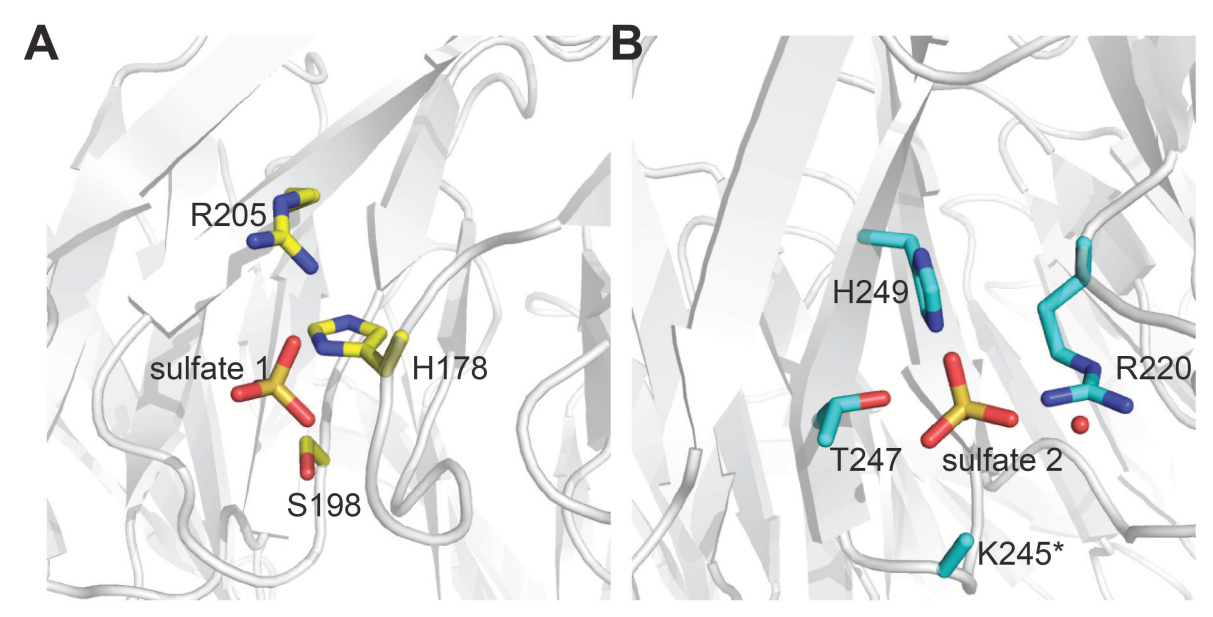

Figure 3.1: Sulfate binding site 1 (A) and binding site 2 (B) in the KIHsv2 structure.

Since sulfates and phosphates bound in crystal structures of PI binding proteins often reveal the positions of phosphates of PI headgroups, we hypothesized that these two sulfates could define either one or two PI binding sites. In the two binding sites hypothesis, the residues around these sulfates defining potential binding site 1 are H178, S198 and R205, while potential binding site 2 is defined by R220, K245, T247 and H249. In that case R219, which is essential for PI binding would need to contribute to sulfate binding site 2. This would make sulfate binding site 2 the single PI binding site. Due to crystal packing contacts, R219 side chain might be trapped in site 1 because of W277 (Figure 3.2). For this hypothesis to be verifiable, R219 must be able to 
swing towards site 2 . This conformation change must be accompanied by the release of W277 from the crystal packing contacts that hinders the move of R219. Movement of W277 might allow R219 to point towards sulfate site 2. In order to test this hypothesis, I performed a $30 \mathrm{~ns}$ MD simulation with the solvated KlHsv2 structure containing the complete loop 6CD. When overlaying the initial crystal structure with the coordinates of the last time point of the simulation, one observes that W277 is released from its restraints by crystal packing contacts, freeing binding site 2. However, R219 remains oriented opposite to R220 (Figure 3.2). These findings support the two PI binding sites hypothesis. Mutagenesis studies confirmed the presence of two PI binding sites [1].

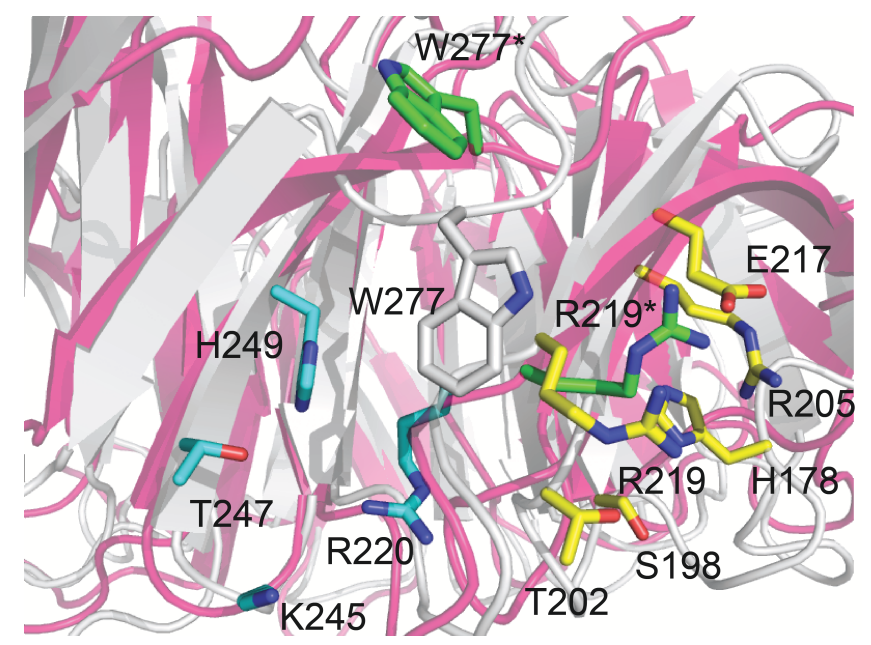

Figure 3.2: W277 release from crystal packing contacts by MD simulation.

KlHsv2 was solvated and simulated for $30 \mathrm{~ns}$. Final structure (pink) is shown overlaid to the initial crystal structure (gray). Binding site 1 is shown in yellow sticks, while binding site 2 is represented by cyan sticks. Final positions of W277 and R219 are depicted in green sticks and labeled with *.

\subsubsection{In silico analysis of phosphoinositides binding of KIHsv2}

\subsubsection{Computational docking of PI3P and PI(3,5)P $P_{2}$ in the two binding sites of KIHsv2}

PI3P and PI(3,5) $\mathrm{P}_{2}$ were docked using AutoDock 4.2 [169] in both sulfate binding pockets (Figure 3.1). PI3P was successfully docked in both binding sites (Figure 3.3). In the optimal docked conformation, H178, S198, D200, T202, R205 and R219 are the essential residues for binding of PI3P in binding site 1. Salt-bridges form between phosphates P1 and P3 and R205 and R219, respectively. Similarly, the essential residues in binding site 2 for PI3P binding are R220, K245, H249 and K283. The salt bridges formed between the ligand and the protein are $\mathrm{H} 249$ and R220 with phosphate P1, while phosphate P3 interacts with K245 and K283. The details of all docking experiments performed are summarized in Appendix table 8.1 for binding site 1, while Appendix table 8.3 summarizes the details for binding site 2 .

Using the same procedure, the binding mode of $\mathrm{PI}(3,5) \mathrm{P}_{2}$ with the two binding sites was determined (Figure 3.4). Interestingly, $\mathrm{H} 178$ and $\mathrm{H} 249$ needed to be protonated for successful docking of $\mathrm{PI}(3,5) \mathrm{P}_{2} \cdot \mathrm{PI}(3,5) \mathrm{P}_{2}$ is recognized by residues in the two binding sites in the same way as PI3P with additional salt bridges between phosphate P5 and H178 for binding site 1 and K283 for binding site 2. The details of all docking experiments performed are summarized in Appendix table 8.2 for binding site 1, while Appendix table 8.4 summarizes the details for binding site 2 . 
A

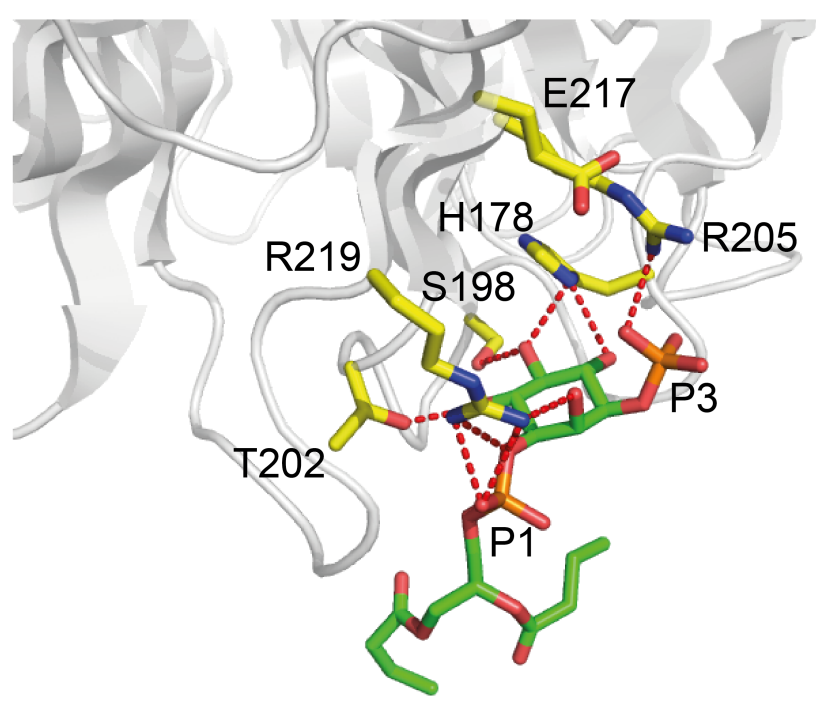

C

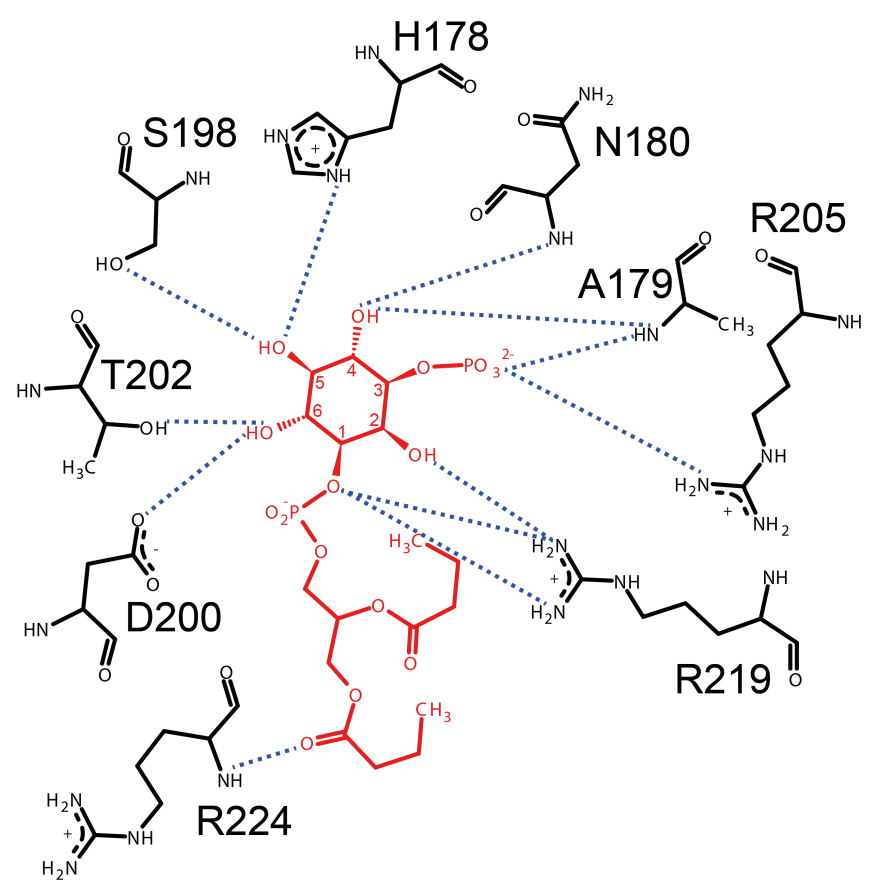

B

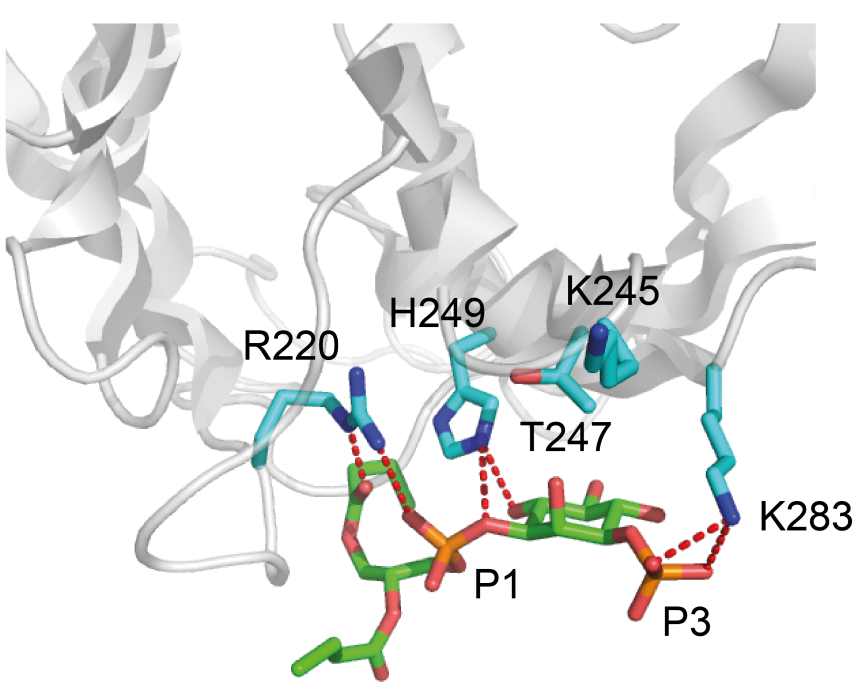

D

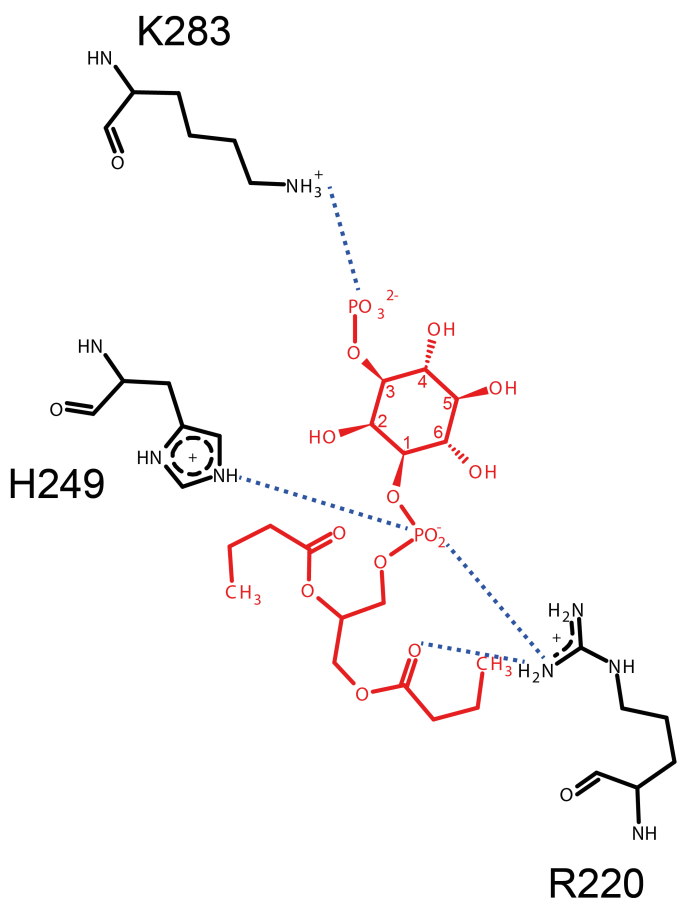

Figure 3.3: PI3P docking in binding sites 1 and 2.

(A) Spatial representation of PI3P in binding site 1 and (B) site 2. The docking was done using AutoDock 4.2 ([169]). PI3P is represented in green sticks. The essential residues in binding site 1 are shown in yellow sticks while the ones in binding site 2 are shown in cyan sticks. Salt bridges and hydrogen bonds are depicted in red dashed lines. (C) Schematic diagram of K1Hsv2-PI3P interaction in binding site 1 and (D) site 2. KlHsv2 residues involved in the ligand interaction are colored black, while PI3P is colored red. Salt bridges and hydrogen bonds are depicted with blue dashed lines. Hydrophobic interactions were neglected for reasons of simplicity. 
A

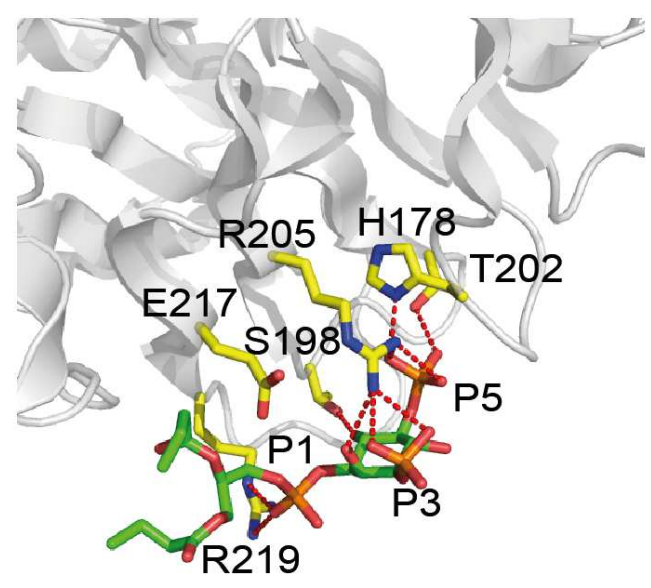

C

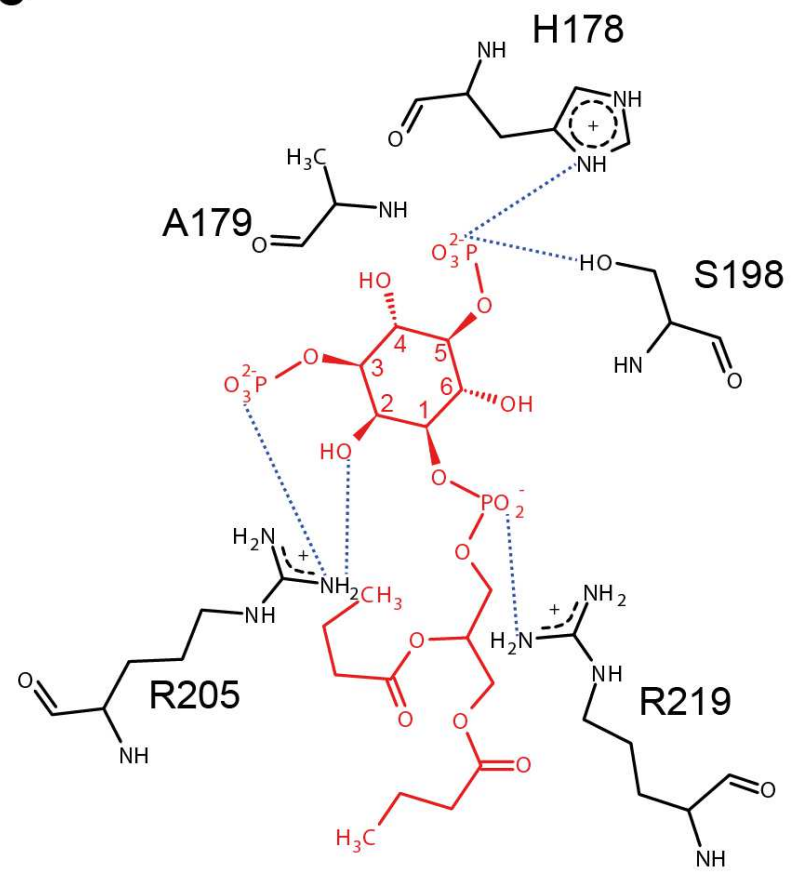

B

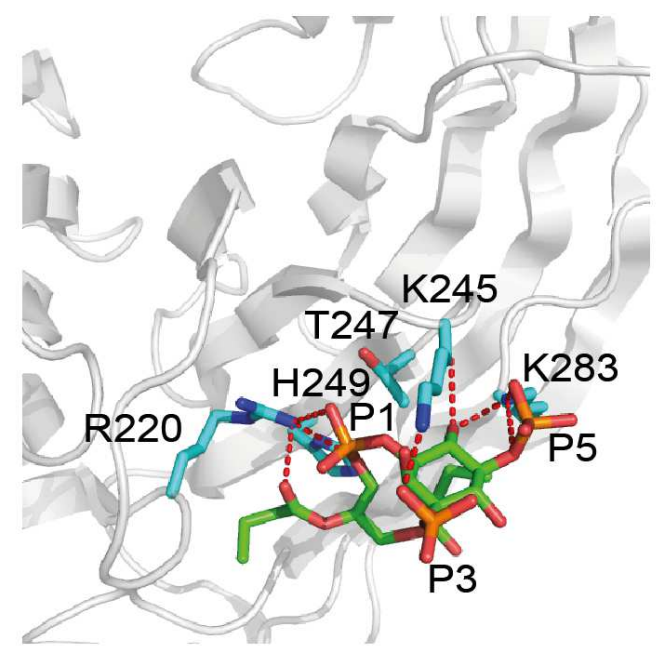

D

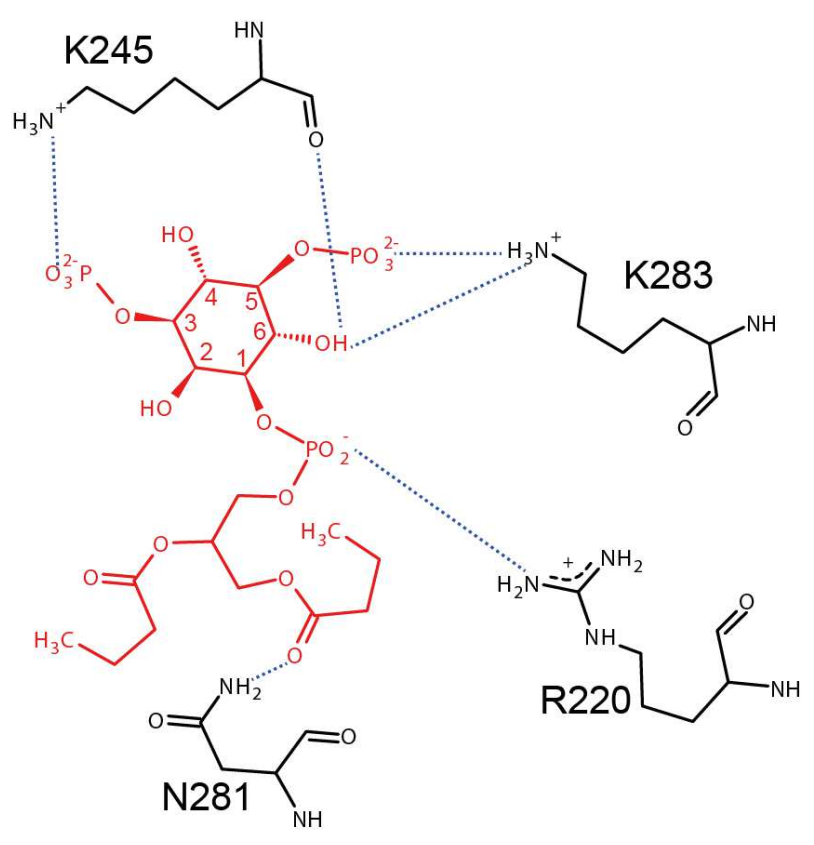

Figure 3.4: PI(3,5) $P_{2}$ docking in binding sites 1 and 2.

(A) Spatial representation of $\mathrm{PI}(3,5) \mathrm{P}_{2} \mathrm{P}$ in binding site 1 and (B) site 2. The docking was done using AutoDock 4.2 ([169]). $\mathrm{PI}(3,5) \mathrm{P}_{2}$ is represented in green sticks. The essential residues in binding site 1 are shown in yellow sticks while the ones in binding site 2 are shown in cyan sticks. Salt bridges and hydrogen bonds are represented in red dashed lines. (C) Schematic diagram of $\mathrm{KIHsv} 2-\mathrm{PI}(3,5) \mathrm{P}_{2}$ interaction in binding site 1 and (D) site 2. KlHsv2 residues involved in the ligand interaction are colored black, while $\mathrm{PI}(3,5) \mathrm{P}_{2}$ is colored red. Salt bridges and hydrogen bonds are depicted with blue dashed lines. Hydrophobic interactions were neglected for reasons of simplicity. 
A summary of all the contacts reveled by all the docking experiments is shown in Table 3.1. This table was obtained by counting how many times a certain contact appears in all the docking experiments.

Table 3.1: Summary of all the contacts, salt-bridges or hydrogen bonds, revealed during the docking experiments as shown in Appendix tables 8.18.4 In parentheses the count of docking conformations in which these contacts are made is given.

\begin{tabular}{|c|c|c|c|c|c|c|c|}
\hline & & P1 & $\mathbf{P 3}$ & $\begin{array}{c}\text { P5 } \\
\text { or OH5 }\end{array}$ & OH2 & OH4 & OH6 \\
\hline \multirow[t]{5}{*}{ PI3P } & binding & R219(8) & R205(21) & $\mathrm{D} 200(7)$ & & & T202(8) \\
\hline & site 1 & & A179(12) & S198(6) & & & $\mathrm{R} 219(6)$ \\
\hline & & & & T202(6) & & & \\
\hline & binding & R220(4) & K283(13) & D244(14) & & & D244(12) \\
\hline & site 2 & & & $\mathrm{~T} 225(6)$ & & & $\mathrm{T} 247(4)$ \\
\hline \multirow[t]{6}{*}{$\mathbf{P I}(\mathbf{3 , 5}) \mathbf{P}_{2}$} & binding & R219(8) & R205(15) & "H178(6) & & $\overline{\text { D200(4) }}$ & \\
\hline & site 1 & & & N180(15) & & & \\
\hline & & & & S198(5) & & & \\
\hline & binding & $\mathrm{T} 247(3)$ & K283(11) & K245(11) & D244(5) & & D244(6) \\
\hline & site 2 & R220(2) & & & & & \\
\hline & & H249(3) & & & & & \\
\hline
\end{tabular}

\subsubsection{Dynamics of the binding sites in the bound and unbound form}

Next, I investigated the strength of the salt bridges formed between the ligands and KlHsv2. In order to do so, three $30 \mathrm{~ns}$ MD simulations were performed for each ligand in each binding site and either two PI3Ps or two $\mathrm{PI}(3,5) \mathrm{P}_{2}$ occupying both binding sites. The time evolution of the distance between the closest protein contacts to the phosphates, P1, P3 and P5 of the ligands was analyzed (Figure 3.5).

A salt-bridge is maintained when the distance between the phosphorous of the studied phosphate and the central atom of the respective side chain is under the threshold of $6 \AA$

In the case of binding site 1, for PI3P,it was observed that R219 makes a strong salt bridge with the P1. This salt bridge is stable over the time of the simulation. In the case of P3, salt bridges are formed with both $\mathrm{H} 178$ and R205, the latter being the weaker one. Interestingly, for $\mathrm{PI}(3,5) \mathrm{P}_{2}, \mathrm{H} 178$ switches from P3 to P5, with whom it makes a stable bond. Similarly, R205 makes a weak salt bridge with P5, and an even weaker salt bridge with $\mathrm{P} 3$. For $\mathrm{PI}(3,5) \mathrm{P}_{2}$, P1 makes a weak salt bridge with $\mathrm{R} 205$, but this gets broken.

When analyzing the situation for binding site 2, for PI3P, P1 makes stable salt bridges with $\mathrm{H} 249$ and R220, while P3 is stabilized by K245 and K283. For PI $(3,5) \mathrm{P}_{2}$, the P1 salt bridge with $\mathrm{H} 249$ is disrupted, while a stable salt bridge with K283 is formed. In the case of P3, the salt bridge with K245 is maintained, while K283 shifts in between P3 and P5. Interestingly, P5 gets stabilized by K285.

Overall, analyzing the trajectory in the case of $\mathrm{PI}(3,5) \mathrm{P}_{2}$, the initial salt bridges with phosphates $\mathrm{P} 1$, namely, R219 and, R220 and H249, respectively, are broken first, allowing the ligands to drift with this phosphate towards the charged residues forming salt-bridges with the other phosphates later leading to the disruption of these electrostatic contacts as well.

Ligand binding usually leads to conformational stabilization of the protein. In order to study whether this is the case in the ligand simulations at hand, the root-mean-square fluctuations (RMSFs) as a function of 

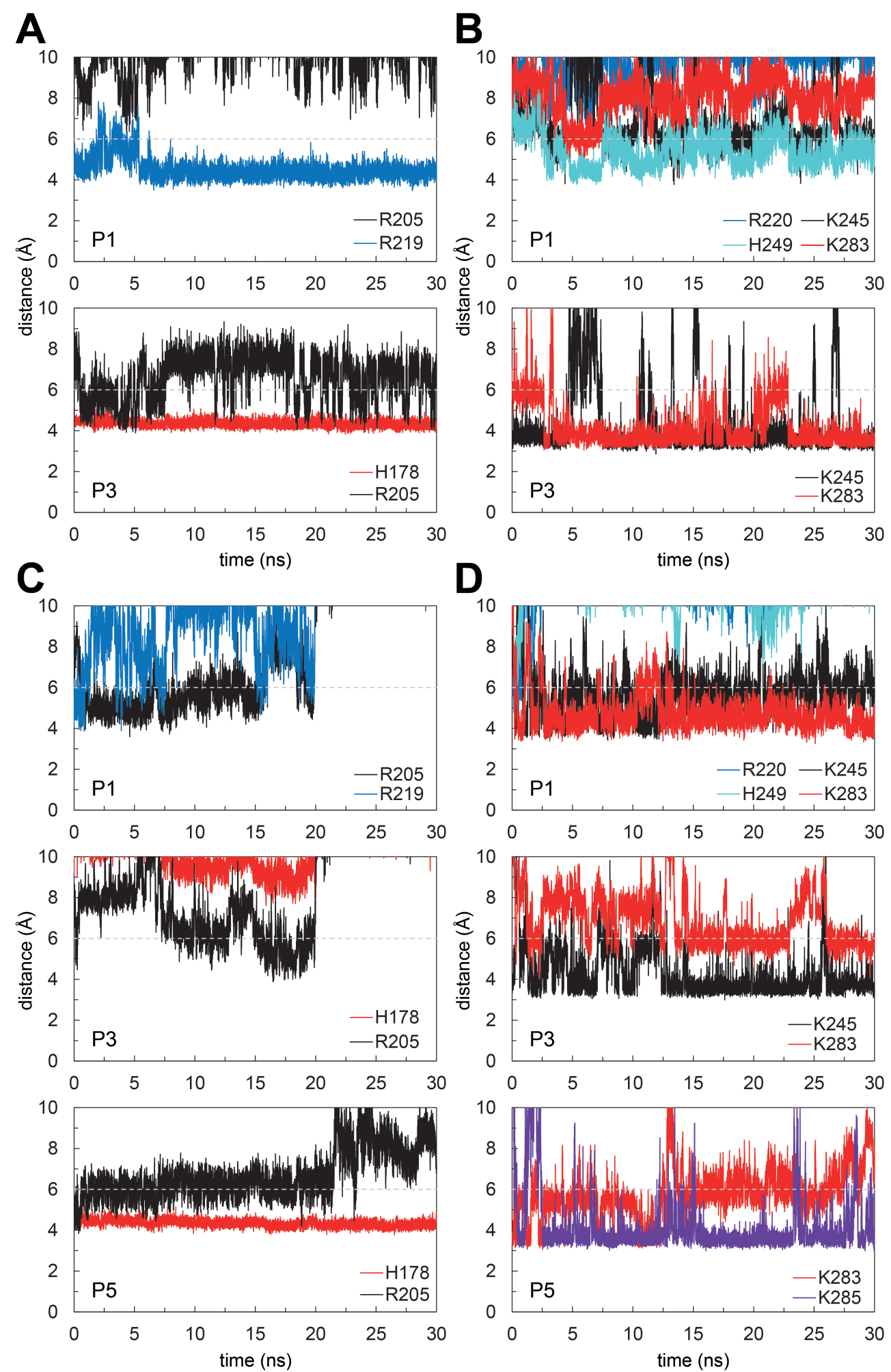

Figure 3.5: Dynamics of the KIHsv2 binding sites in the bound and unbound form.

Residue-phosphate distances over time for the simulations with two PI3P or PI(3,5) $\mathrm{P}_{2}$ in both binding sites of KlHsv2. Distances are measured between the phosphorus atom and the CZ (for arginines), ND1/NE1 (for histidines) or NZ (for lysines) atoms of interacting basic residues. The interaction limit set to $6 \AA$ is shown as a dashed grey line. (A) Distances are shown for P1 and P3 of PI3P in binding site one. (B) Distances are shown for P1 and P3 of PI3P in binding site two. (C) Distances are shown for P1, P3 and of PI $(3,5) \mathrm{P}_{2}$ in binding site one. (D) Distances are shown for P1, P3 and P5 of PI $(3,5) \mathrm{P}_{2}$ in binding site two. 
residue number of both the apo-protein simulations and the ligand simulations were analyzed (Figure 3.6). The fluctuations are comparable in most of the parts of the protein, except the loop $6 \mathrm{CD}$ which is stabilized upon ligand binding. Moreover, during the apo-protein simulation, this loop adopts different folds: $\alpha$-helix, $\pi$-helix, $3_{10}$-helix and coil.

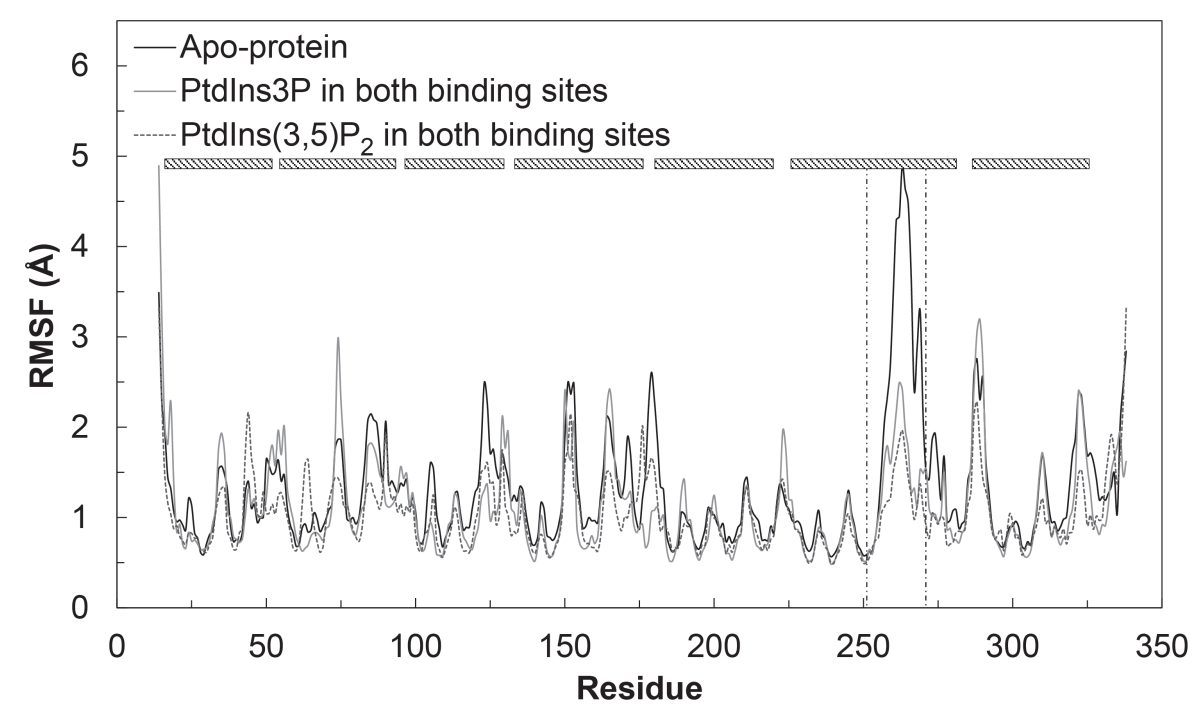

Figure 3.6: Root-mean-square fluctuations (RMSFs) of the $\mathrm{C} \alpha$ atoms as a function of residue number.

RMSFs were calculated from the last $22 \mathrm{~ns}$ of the $30 \mathrm{~ns}$ molecular dynamics simulations of Apo-K1Hsv2, PI3P and PI(3,5) $\mathrm{P}_{2}$ in both binding sites of K1Hsv2. The rectangular boxes represent the seven blades of the $\beta$-propeller, while the dashed vertical lines show the loop 6CD.

\subsubsection{Phosphoinositides membrane binding of ScHsv2 and KlAtg21}

The previous sections analyzed the PI binding sites for KlHsv2. In order to gain deeper insights in the PI recognition by PROPPINs, especially by the PROPPINs used in this thesis, I used homology modeling based on the experimentally determined structure of KlHsv2 [1].

The first step in homology modeling is a sequence alignment of the target sequence to the sequence of the template structure. Figure 3.7 shows the multiple sequence alignment of textitK. lactis Hsv2 (K1Hsv2), $S$. cerevisiae Hsv2 (ScHsv2), S. cerevisiae Atg18 ScAtg18, P. angusta Atg18 (PaAtg18), C. thermophilum Atg18 (CtAtg18), S. cerevisiae Atg21 (ScAtg21) and K. lactis Atg21 (K1Atg21). One observes that, due to the fact that loop 6CD is not conserved neither in sequence, not in length, the alignment is not easily made by automatic servers. For example, K283 in K1Hsv2, even though aligns with $\mathrm{H} 345$ in its S. cerevisiae homolog, does not align with the well conserved similar patch - as defined by K416 in ScAtg18 and T447 in ScAtg21 - in the other PROPPINs. This has to be done manually. The essential residues for PI binding in these PROPPINs are summarized in Table 3.2

ScHsv2 and KlAtg21 were chosen for homology modeling. The resulting models are presented in Figure 3.8 Particular attention is given to the two PI binding sites. In the case of ScHsv2, the essential residues in binding site 1 are H223, R250 and R264, while for the binding site 2, R265, K290, H294 and H345 are important. Interestingly, ScHsv2 has an extra histidine in its binding site 2. In the case of KlHsv2 this residue, 


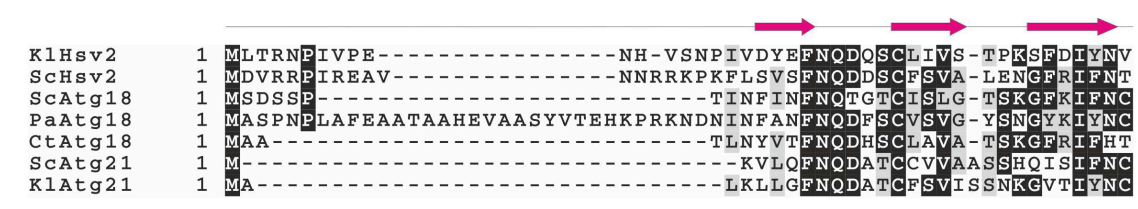

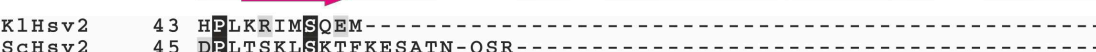

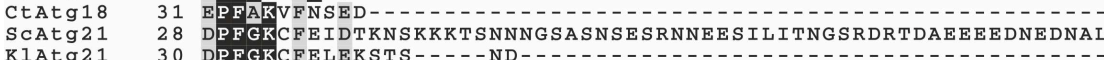
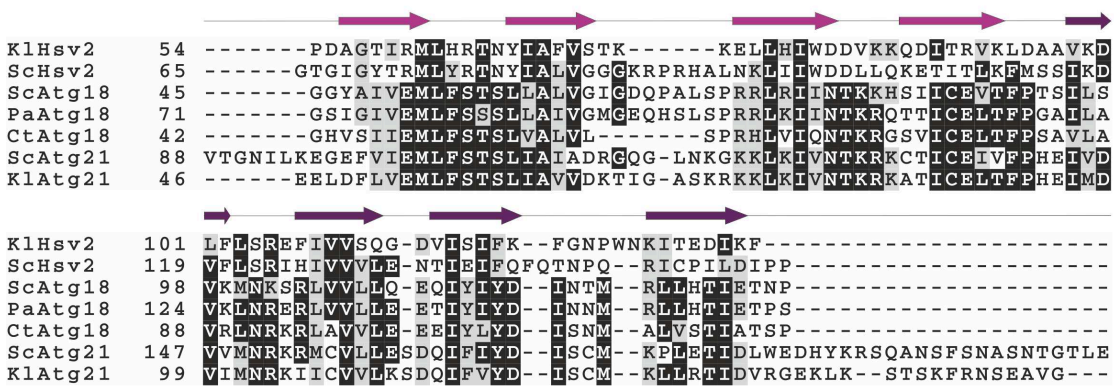

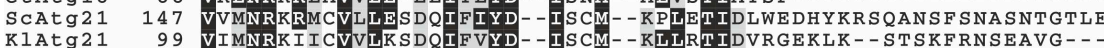

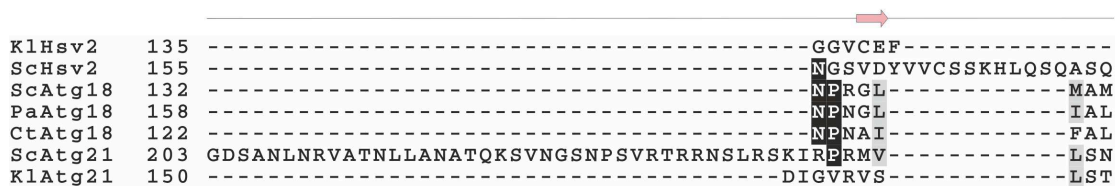

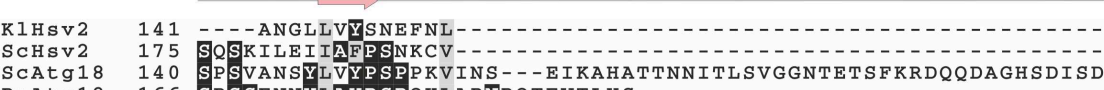

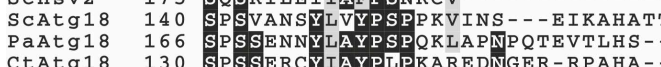

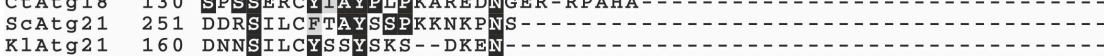

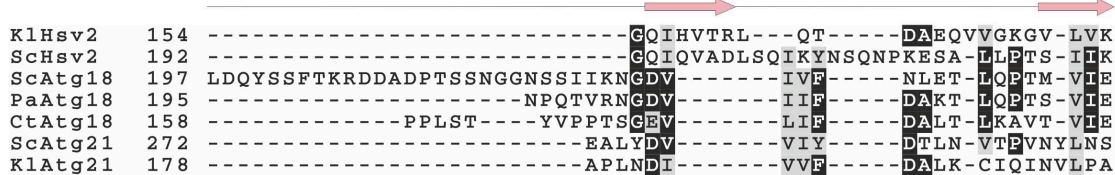

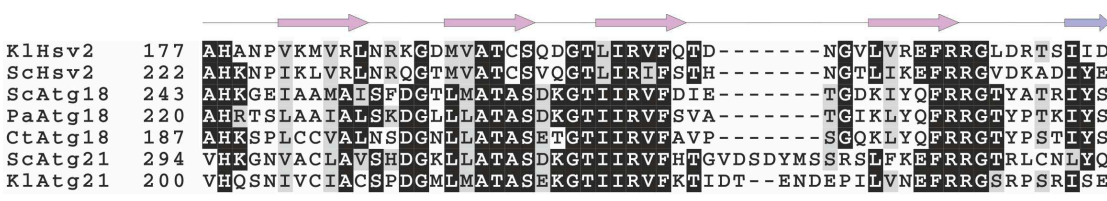

$\longrightarrow \vec{P}$

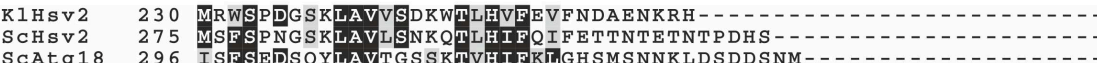

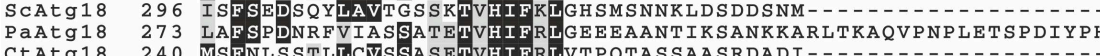

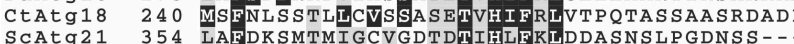

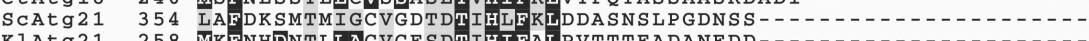

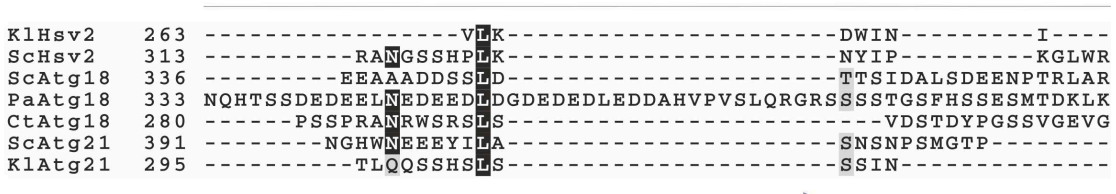

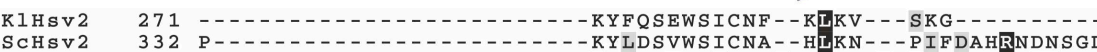

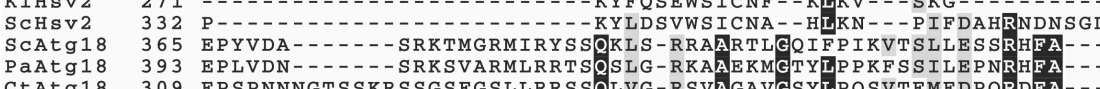

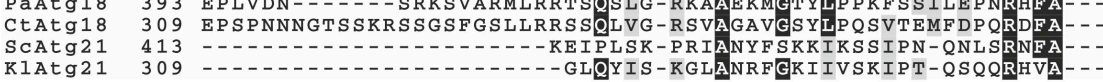
K1Atg21 309 -

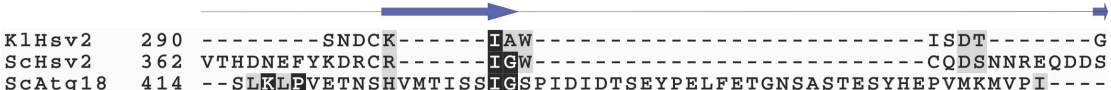

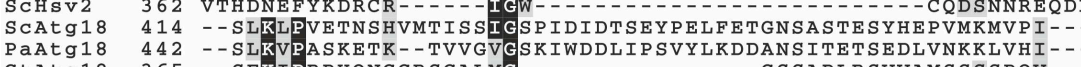

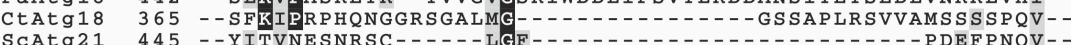

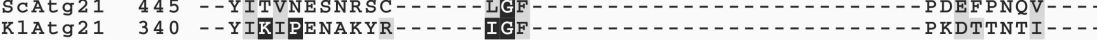

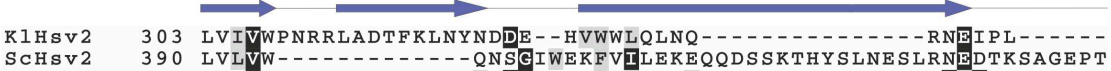

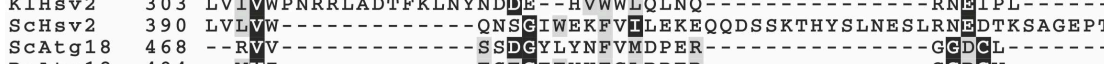

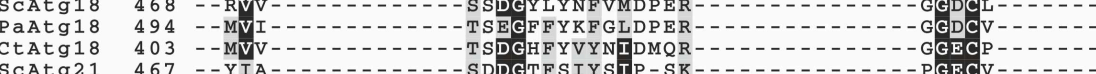

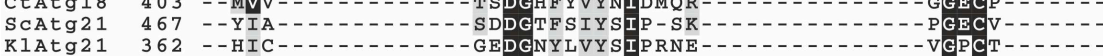

K1Hsv2

CHSV 2437 RWELVRESWREL-

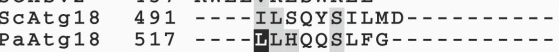

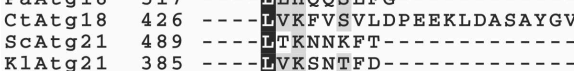

\section{Figure 3.7: Multiple sequence alignment of PROPPINs.}

K. lactis Hsv2 (KlHsv2), S. cerevisiae Hsv2 (ScHsv2), S. cerevisiae Atg18 ScAtg18, P. angusta Atg18 (PaAtg18), C. thermophilum Atg18 (CtAtg18), S. cerevisiae Atg21 (ScAtg21) and K. lactis Atg21 (KIAtg21) were aligned using T-Coffee [185] server. Secondary structure elements are shown based on the determined structure of KIHsv2 [1]. 
Table 3.2: Comparison of important residues for PROPPINs forming salt bridges with phospoinosites across paralogs of several yeast species.

\begin{tabular}{ccccccccc}
\hline & & \multicolumn{3}{c}{ Binding site 1 } & \multicolumn{4}{c}{ Binding site 2 } \\
\hline PROPPIN & Species & P5 & P3 & P1 & P1 & P5 & P1 & P3 \\
\hline Hsv2 & K. lactis & H178 & R205 & R219 & R220 & K245 & H249 & K283 \\
& S. cerevisiae & H223 & R250 & R264 & R265 & K290 & H294 & H345 \\
\hline Atg18 & S. cerevisiae & H244 & R271 & R285 & R286 & S311 & H315 & K416 \\
& P. angusta & H221 & R248 & R262 & R263 & T288 & H292 & K444 \\
\hline Atg21 & S. cerevisiae & H295 & R322 & R343 & R344 & T369 & H373 & T447 \\
& K. lactis & H201 & R228 & R247 & R248 & S273 & H277 & K342 \\
\hline
\end{tabular}
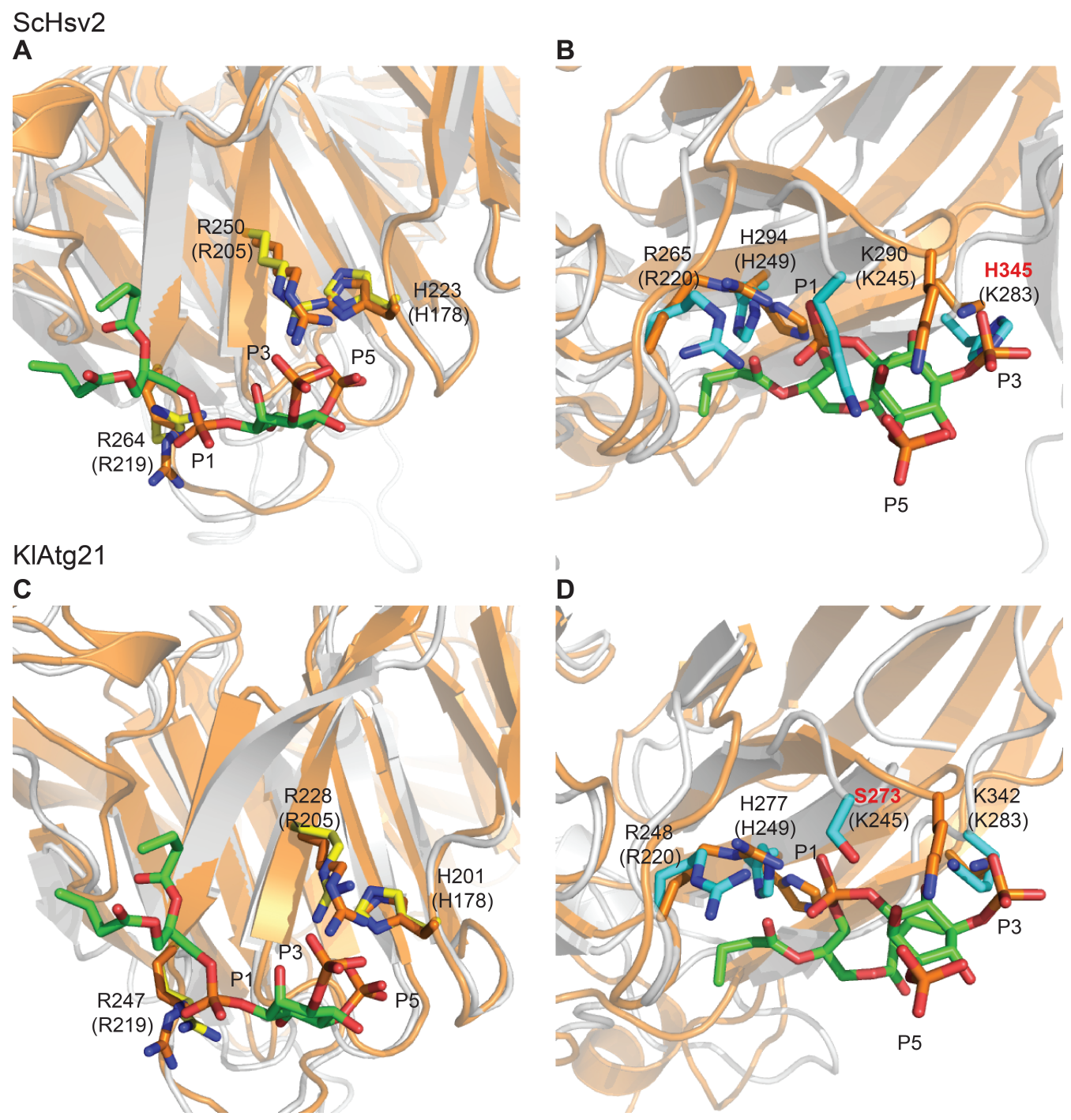

Figure 3.8: Essential residues in PI binding for ScHsv2 and KIAtg21 as revealed by homology modeling.

(A) Spatial representation of $\mathrm{PI}(3,5) \mathrm{P}_{2} \mathrm{P}$ in binding site 1 and (B) site 2 for the homology model of ScHsv2, and (C) binding site 1 and (D) site 2 for the homology model of KlAtg21. The homology models are represented in gray cartoons while the KlHsv2 structure, used as template for modeling is represented in orange. The KlHsv2 residues important for PI binding are represented in orange sticks. $\mathrm{PI}(3,5) \mathrm{P}_{2}$ is represented in green sticks. For the homology models, the essential residues in binding site 1 are shown in yellow sticks while the ones in binding site 2 are shown in cyan sticks. 
making a salt bridge to phosphate 3 of the inositol ring, is a lysine. In the KlAtg21 case, binding site 1 is also well conserved, with H201, R228 and R247 being important, while in the binding site 2, the essential residues are R248, S273, H277, K342. While binding site 1 is well conserved across species, as shown in Table 3.2, binding site 2 shows less conservation. While for $\mathrm{ScHsv} 2$, the difference was one basic amino acid (lysine) being changed to another basic amino acid (histidine), in the case of Atg18s and Atg21s, a lysine in KlHsv2 is exchanged to a serine or threonine - polar residues.

\subsection{Unspecific membrane binding of KlHsv2}

Once the specific membrane binding by PROPPINs through PI3P and $\mathrm{PI}(3,5) \mathrm{P}_{2}$ recognition was computationally studied, the next question was whether PROPPINs do not have other structural features that would help in the stabilization of the binding or recruitment to the membrane, as observed in other PI binding domains.

\subsubsection{Preliminary model of KlHsv2 membrane binding}

Having the KlHsv2-PI3P bound conformation for both biding sites from Figure 3.3, I proposed a model for KlHsv2 membrane recognition (Figure 3.9). By defining the membrane as formed by the horizontal plane cutting through the two P1 phosphates of the PI3Ps bound to the two binding sites, there can be observed two loops, 6CD and 7CD which insert in the bilayer. Moreover, the PROPPIN sits perpendicular on the membrane, with its toroidal axis parallel to the membrane.

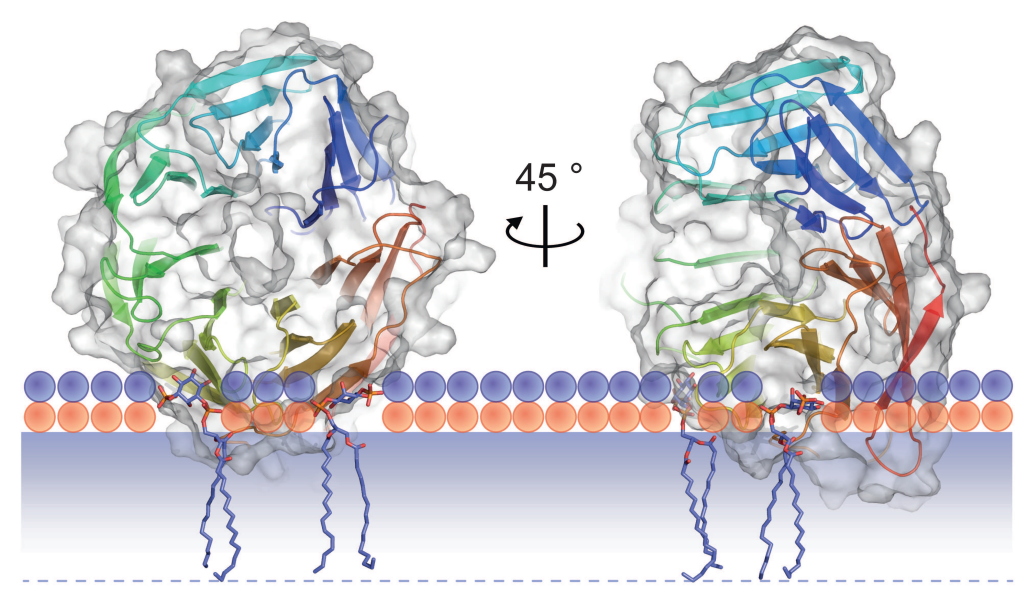

Figure 3.9: Preliminary model for membrane recognition by KIHsv2

The two PI3Ps are shown in blue sticks. The positions of the phosphates of the membrane phospholipids are shown through orange circles, while the blue circles represent the polar groups of these phospholipids. The shaded violet bar depicts the fatty acid tails of the phospholipids from the cytosolic leaflet of the bilayer. The dashed blue line represents the center of the bilayer. The model was approximately drawn to scale. Loops 6CD and 7CD insert inside the membrane according to this model.

\subsubsection{Membrane insertion of loop 6CD based on molecular dynamics simulations}

The preliminary model described in the previous section is based on empirical methodology. More advanced methodology was used to study whether there is membrane loop insertion. This is why, I have investigated the propensity of the independent insertion of loop 6CD into a membrane using a protocol [178] 
developed for monotopic membrane proteins. In short, a coarse grained molecular dynamics (CG-MD) simulation is performed to form a membrane around the exposed hydrophobic parts of the protein. For CG-MD simulations, four atoms are approximated to a bead. This protocol has the advantage that will give an unbiased orientation of the protein with respect to the membrane. However, due to the approximations of the coarse grained model used, phosphoinositides cannot be modeled accurately.

The first steps in performing an CG-MD with a protein is to check which representation is better suited for the system. There are three possible protocols for obtaining a coarsed-grained protein: Martini [189], Martini combined with elastic network [189] and ElNeDyn [190]. Besides the protein representation, the time step should also be optimized. Two time steps were chosen, $20 \mathrm{ps}$ and $40 \mathrm{ps}$. The three models were probed and compared with an atomistic simulation for $100 \mathrm{~ns}$ in similar conditions. The two analyzed properties used for this comparison were the RMSD - for deviations from the original structure - and the RMSF - for fluctuations of $\mathrm{C} \alpha$ atoms. The ElNeDyn protocol failed for the case of KlHsv2, but the results for Martini and Martini combined with elastic network are shown in Figure 3.10 On one hand, simple Martini protocol led to high deviations from the original structure for both time steps chosen, as the RMSD values were above $15 \AA$, while the RMSFs of the $\mathrm{C} \alpha$ atoms were also large compared to the atomistic simulation. On the other hand, when combined with an elastic network, Martini was suitable to represent the protein in a coarse-grained model. A smaller time step performed more similar to the atomistic simulation in terms of RMSDs, while the RMSFs looked similar to the atomistic simulation independent of the time step chosen. This is why the Martini combined with an elastic network together with a 20 ps time step were chosen for the CG-MD simulations.

Once the protocol for the coarse-grain representation of KlHsv2 was chosen, the aggregation of DPPC molecules in a bilayer around the protein was performed. Figure 3.11 shows the time evolution of one of the fifteen CG-MDs performed. Initially, Dipalmitoylphosphatidylcholine (DPPC) molecules are randomly positioned in a water box having the protein in the center. During the first $10 \mathrm{~ns}$, the bilayer forms around the loop $6 \mathrm{CD}$. In contrast to the model containing the phosphoinositides, the protein does not position perpendicularly with respect to the membrane, but stays tilted. Moreover, the loop does not penetrate the membrane more than the hydrophilic layer, even though the previous model has shown a deeper penetration. The level of the penetration does not change throughout the remaining $90 \mathrm{~ns}$ of the simulation in which the protein mainly freely rotates around the loop.

Out of the fifteen CG-MD simulations performed, the membrane bilayer formation was observed only six times. This is likely due to the high water to lipid molecules ratio. This high ratio between the water and lipid molecules was necessary because of the considerable size of the protein and necessary size of the simulation box that would fit such a protein. Moreover, out of these six membrane bilayer formations, only in two cases protein inserted in the membrane. This can be explained by the fact that in the other cases, the bilayer formed on the opposite side of the protein and not in the proximity of loop 6CD. Moreover, in some cases in which the protein stayed in solution, the hydrophobic tails of one DPPC molecule stuck to the loop.

In order to facilitate the bilayer formation and not increase the water to lipid ratio, the size of the system was kept low. In fact, the system size did not respect the minimum-image convention and this might be a reason why the protein appeared tilted. The minimum-image convention is needed for the simulated molecules not to be overcrowded, i.e., interact with its own image in the neighboring simulation box. In order to investigate this possibility, the protein-membrane system were placed in a higher box (12 nm on the z-axis) and a $1 \mu$ s CG-MD was performed (Figure 3.12). During the first $50 \mathrm{~ns}$, the protein rotates decreasing the tilt angle. Figure 3.14 , 
A

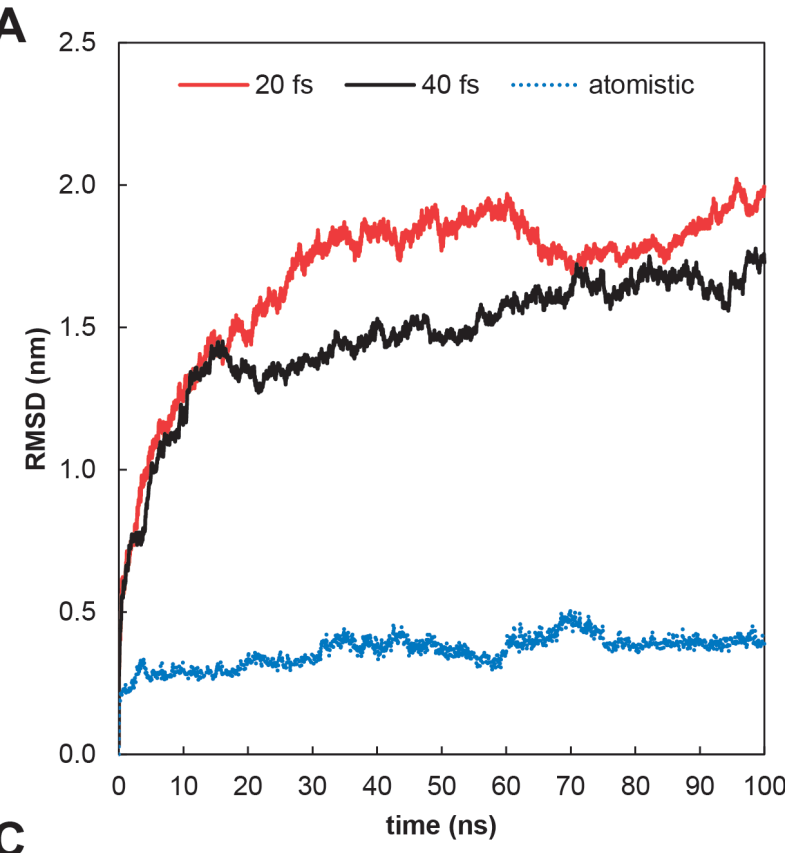

C

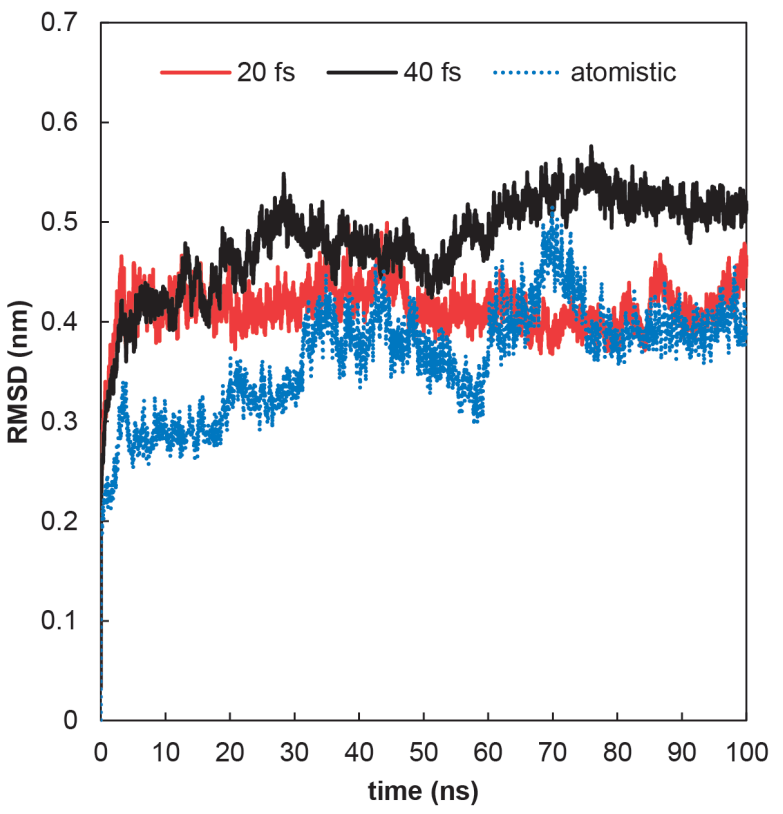

B

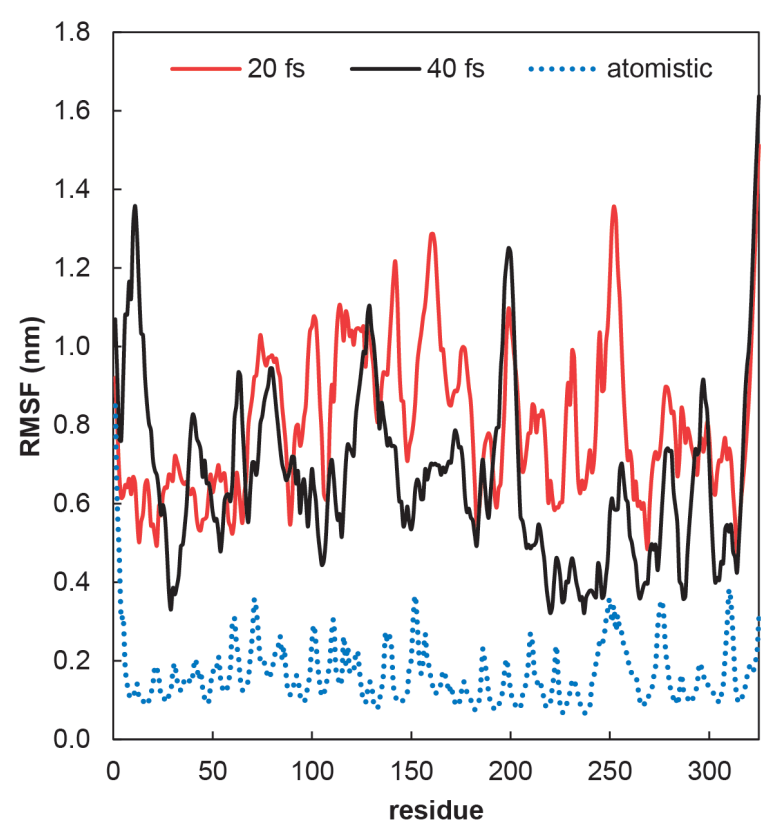

D

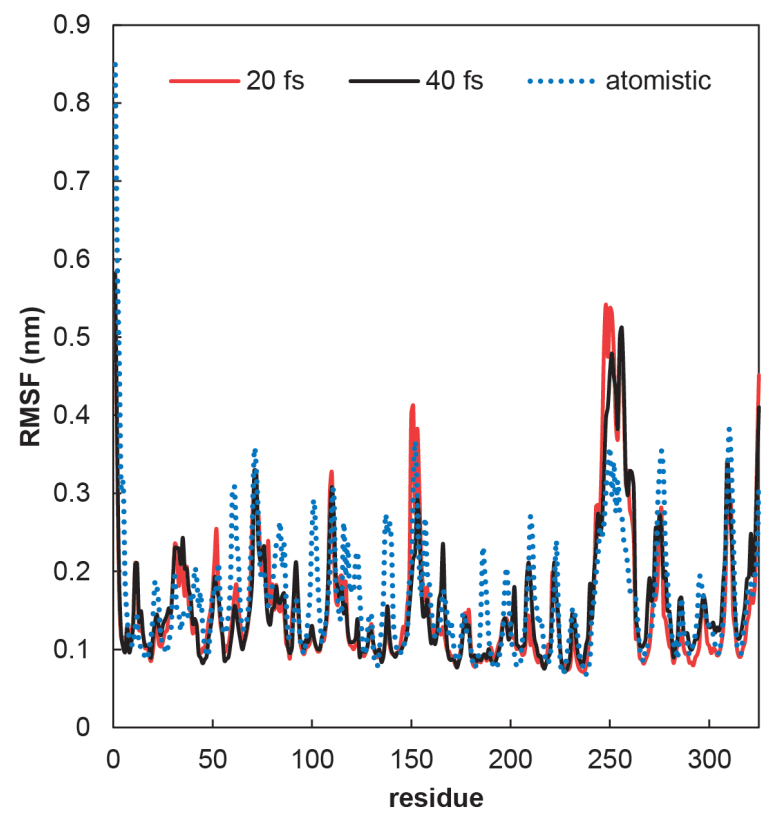

Figure 3.10: Optimization of the coarse-grained representation for KlHsv2.

Comparison of $100 \mathrm{~ns}$ CG-MD simulations using simple Martini protocol with an atomistic simulation in terms of RMSD (A) and RMSF of the $\mathrm{C} \alpha$ atoms (B) for time steps of $20 \mathrm{ps}$ and 40 ps. Comparison of $100 \mathrm{~ns}$ CG-MD simulations using a Martini protocol combined with an elastic network with an atomistic simulation in terms of RMSD (C) and RMSF of the C $\alpha$ atoms (D) for time steps of $20 \mathrm{ps}$ and $40 \mathrm{ps}$. 

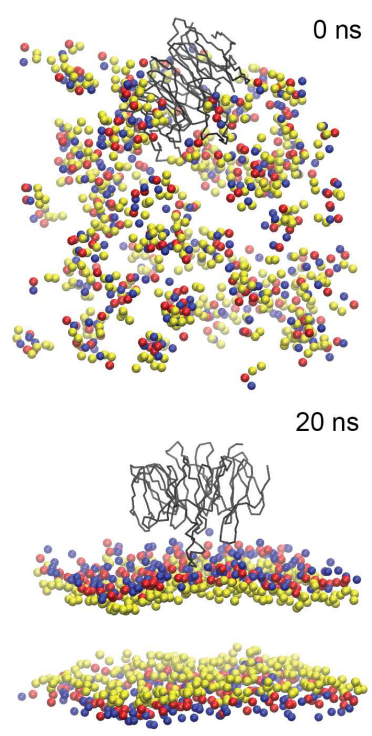
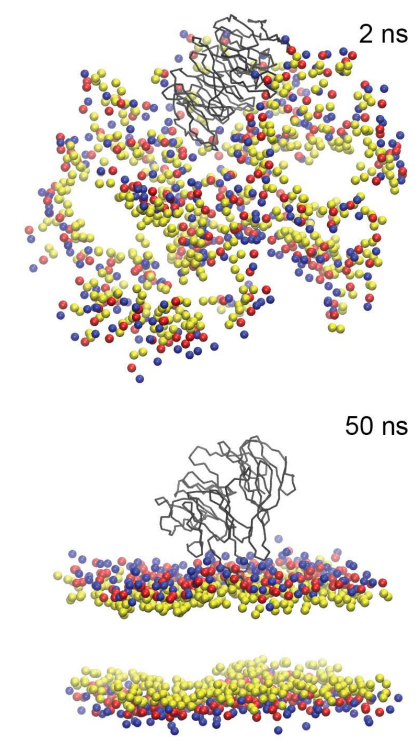
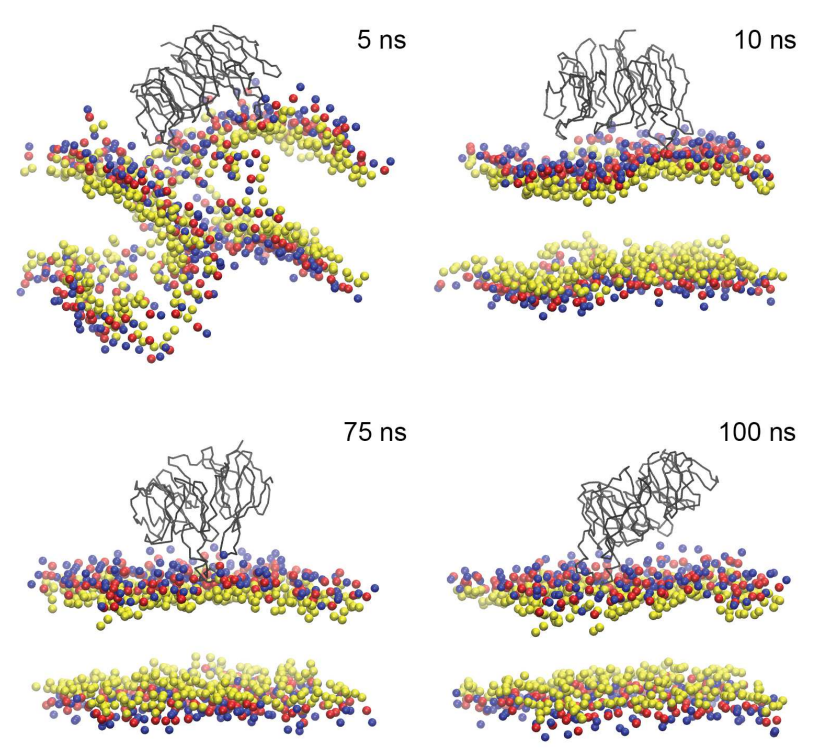

Figure 3.11: Time course of a coarse-grained molecular dynamics simulation of DPPC (Dipalmitoylphosphatidylcholine) bilayer formation around loop 6CD of $K$. lactis Hsv2.

The choline groups of the DPPC are represented in blue, while the phosphates are represented in red and the glycerols in yellow. For the sake of simplicity, the water molecules and the hydrophobic chains of the lipids are not shown.

A shows the time evolution of the tilt angle defined by the protein axis drawn through the backbone of residues T91 and V251 and the z-axis of the system. The average tilt angle is around $30^{\circ}$.

Due to the limitations of the CG-MD simulations, namely the constraints on the secondary structure of the protein and the underestimation of the polarity effects in between water and membrane ([191]), the degree of membrane penetration of loop 6CD could be underestimated. Moreover, the conformation of loop 6CD might be different in solution and in the membrane environment. However, this cannot be accounted for in a CG-MD simulation in which the conformation of a loop is locked since the beginning of the CG-MD. This is why, I performed a $100 \mathrm{~ns}$ atomistic MD simulation for both the protein-membrane system and the protein in solution. The time evolution of this MD simulation is shown in Figure 3.13 .

During the course of the atomistic simulation, the loop 6CD penetrates the membrane deeper, while the protein tumbles around this loop, side-to-side, bringing the arginines of the binding sites closer to the membrane. Moreover, blades 4, 5 and 7 get alternatively in contact with the membrane bilayer as seen in Figure 3.14. B and $\mathrm{C}$. The number of contacts made by each protein residue with the hydrophobic core of the membrane are calculated per ps as shown in Figure 3.14, B. Loop CD is the part that is the most in contact with the hydrophobic parts of the membrane, while residues around the binding site 1, defined by R219, also make contacts with the membrane. Moreover, the protein freely tumbles around loop 6CD such that blades 4 and 7 also get in contact with the membrane. Interestingly, binding site 2, as defined by R220, is never in contact with the membrane. This might suggest that the first binding site that binds a phosphoinositide is binding site 1 , which is the more conserved of the two binding sites.

When the angle made by the protein axis as defined by the $\mathrm{C}$ atoms of residues T91 and V251 with the $\mathrm{z}$-axis of the system is observed across the simulation (Figure 3.14, A), due to the deeper insertion of the loop 

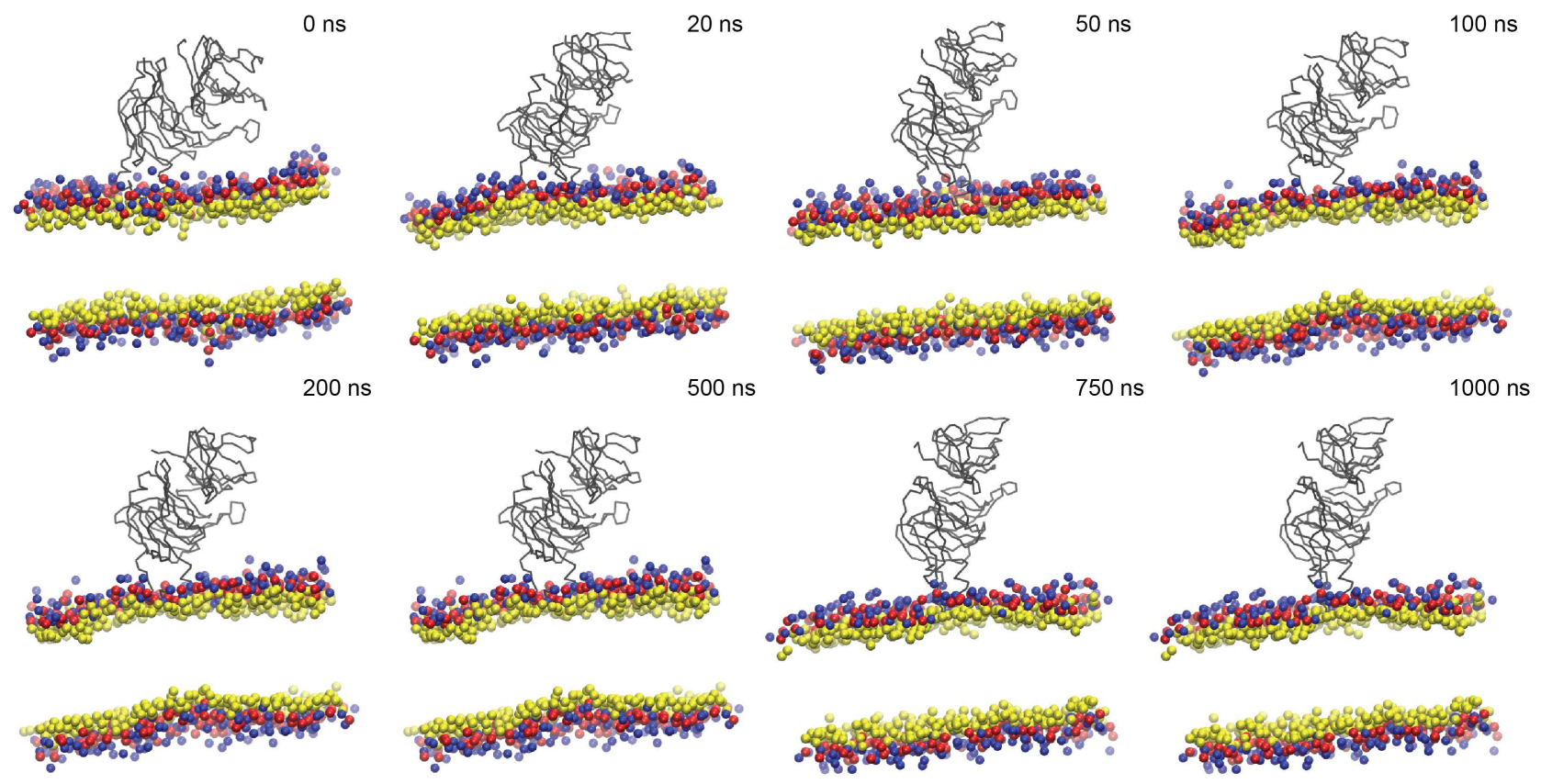

Figure 3.12: Time course of a $1 \mu$ s coarse-grained molecular dynamics simulation of KIHsv2 in a DPPC (Dipalmitoylphosphatidylcholine) membrane.

The choline groups of the DPPC are represented in blue, while the phosphates are represented in red and the glycerols in yellow. For the sake of simplicity, the water molecules and the hydrophobic chains of the lipids are not shown.
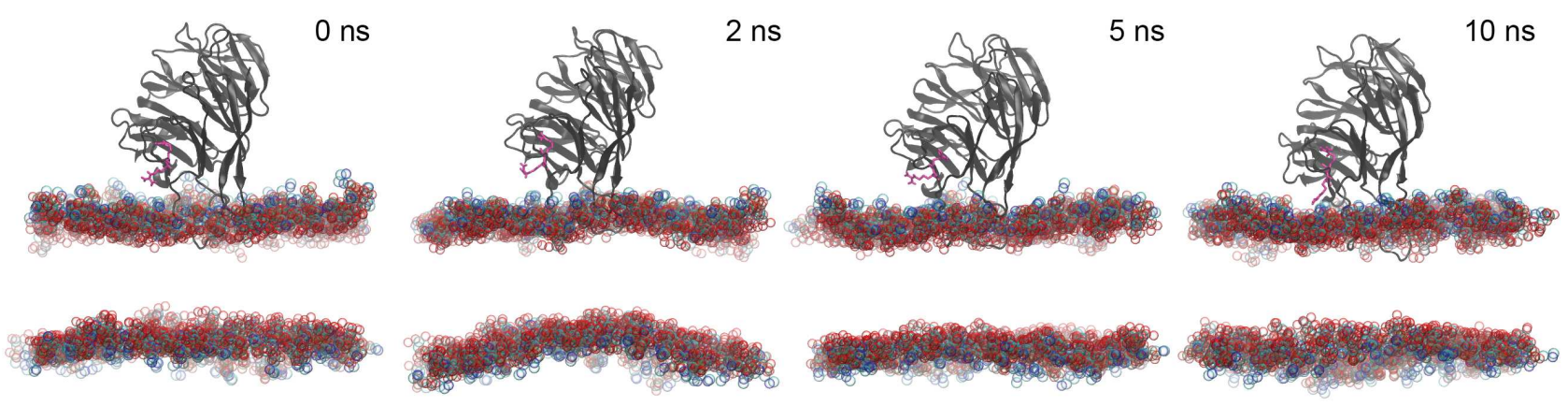

$20 \mathrm{~ns}$
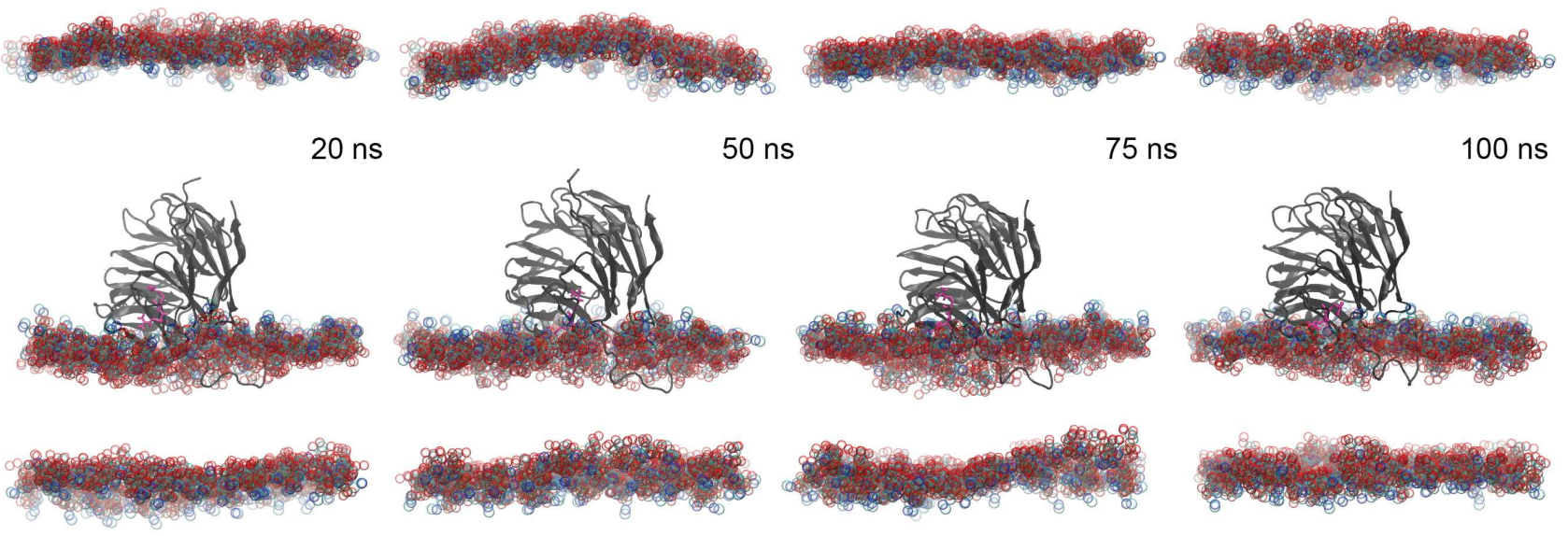

Figure 3.13: Time course of a 100 ns atomistic molecular dynamics simulation of KIHsv2 in a DPPC (Dipalmitoylphosphatidylcholine) membrane.

The oxygen atoms of the DPPC are represented in red, while the nitrogens are dark blue, phosphorous are brown and the carbons light blue. The two arginines defining the binding sites, R219 and R220 are represented in magenta sticks. For the sake of simplicity, the water molecules and the hydrophobic chains of the lipids are not shown. 
A

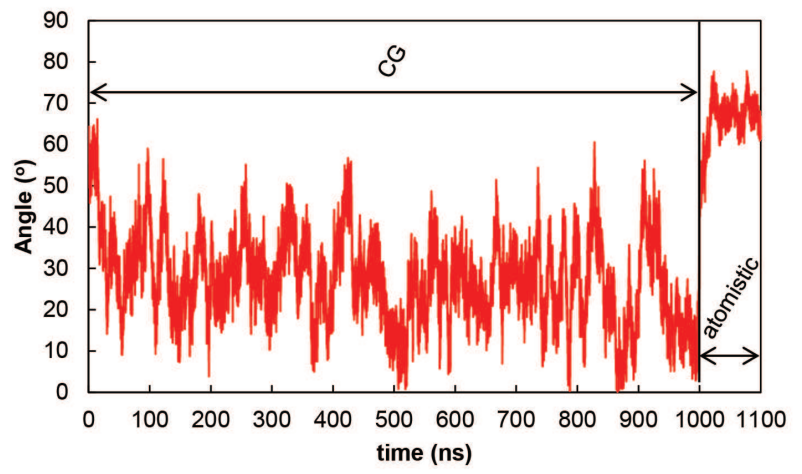

C
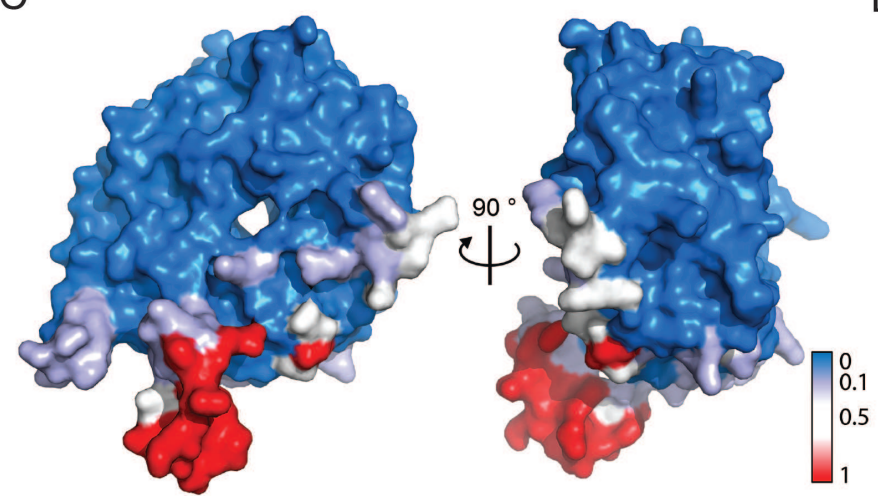

B
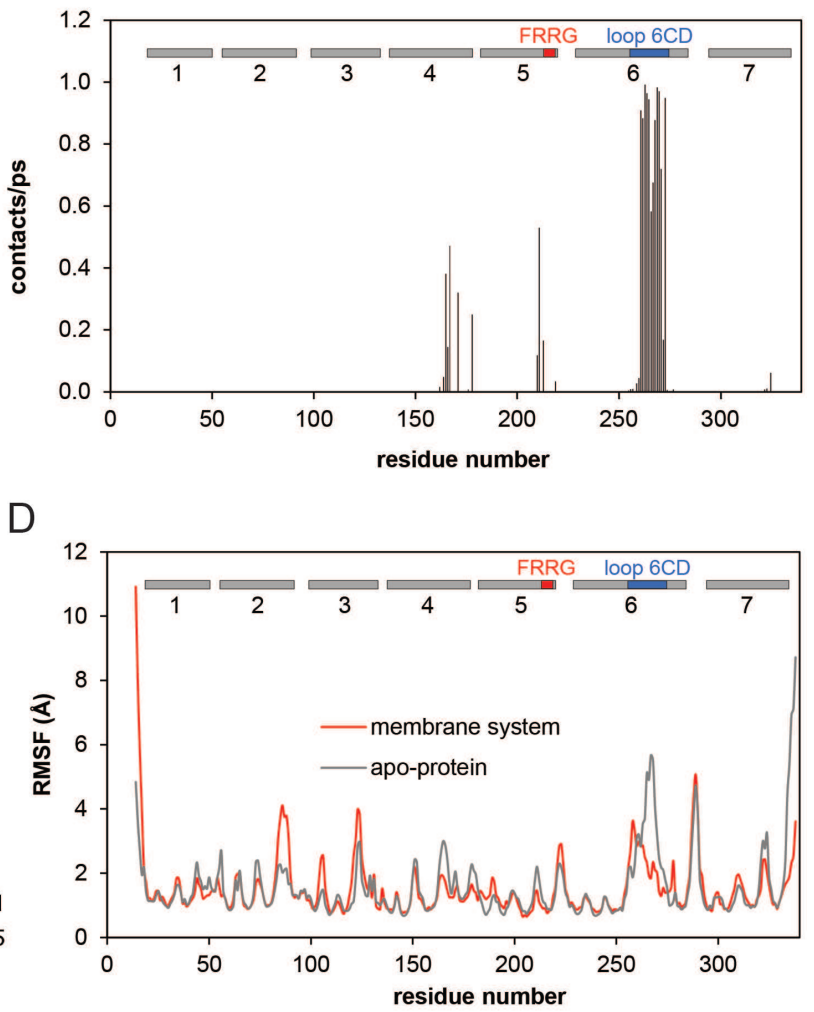

Figure 3.14: Analysis of the molecular dynamics simulations of KIHsv2 in a DPPC (Dipalmitoylphosphatidylcholine) membrane.

(A) The angle between the z-axis of the system and the protein axis defined by the backbone beads (coarse-grained MD simulation) or the $\mathrm{C} \alpha$ atoms (atomistic MD simulation) of T91 and V251 over time. Both the coarse-grained and the atomistic simulation following are shown on the same timeline. (B) Number of contacts each protein residue makes with the carbon tails of the membrane per ps averaged over the $100 \mathrm{~ns}$ atomistic MD simulation. A contact is defined as made when the distance between the heavy atoms of the protein and the heavy atoms of the carbon atoms of the hydrophobic tails of the membrane less than $4 \AA$. The rectangular boxes represent the seven blades of the $\beta$-propeller. (C) Regions of the KlHsv2 that are in contact with the hydrophobic membrane carbon chains. Red represents the regions that make in between 0.5 and 1 contacts per ps over the $100 \mathrm{~ns}$ atomistic MD simulation, while in white are represented those regions that make less than 0.5 , but more than 0.1 contacts per ps. Light blue represents the regions that seldom insert towards the hydrophobic core of the membrane, while the regions that never touch the hydrophobic parts of the membrane are shown in blue. A contact is defined as made when the distance between the heavy atoms of the protein and the heavy atoms of the hydrophobic membrane carbon tails is less than $4 \AA$. (D) Root-mean-square fluctuations (RMSFs) of the C $\alpha$ atoms as a function of residue number of membrane system vs apo-protein $100 \mathrm{~ns}$ simulations. The rectangular boxes represent the seven blades of the $\beta$-propeller. 
$6 \mathrm{CD}$, it also changes, making the protein tilted with the opposite side of the propeller from the binding sites getting closer to the surface of the membrane. Moreover, due to the membrane environment, the root-square fluctuations in the loop 6CD decrease compared with the protein in solution system Figure 3.14, D). 


\section{Biochemical characterization of the phosphoinositides binding specificities of PROPPIN family members}

\subsection{Expression and purification of PROPPINs}

In order to biochemically study PROPPINs, their expression and purification protocols had to be developed. In this chapter, results on the S. cerevisiae Atg18 (ScAtg18) and Atg21 (ScAtg21) expression and purification trials will be reported. Next, based on the doctoral thesis of Dr. Ricarda Busse [192], the P. angusta Atg18 (PaAtg18) and K. lactis Atg21 (KlAtg21) expression and purification protocols were optimized. Furthermore, the expression of $C$. thermophilum Atg18 was also tried in E. coli.

\subsubsection{Expression and purification of S. cerevisiae Atg18}

ScAtg18 is one of the central molecular players in yeast autophagy. Numerous scientific reports $[136,103$, 104, 94, 95, 79] characterize the function of Atg18 using in vivo experiments. However, studying a protein in vitro, has the advantage of isolating its function from the effect of other cellular factors as done in previous studies [104, 93, 94]. In all these cases, ScAtg18 was purified either using a GST or a MBP tag. However, none of the biochemical experiments described needed mg-scale amounts of the proteins.

This is why I have tried to further optimize the expression of ScAt18 using the plasmid kindly provided by the Thumm laboratory [94]. This plasmid expresses ScAtg18 as a fusion with a N-terminal GST tag. The tag can be cut using Prescission protease. The gene sequence encoded was amplified from S. cerevisiae, hence it contained the wild type gene sequence with the codon usage specific for the budding yeast. The promoter used for transcription is the tac promoter, which can be induced by both IPTG or lactose as in the case of auto-inducing media [193]. This promoter can be expressed only in cells containing a T7 RNA polymerase gene such as E. coli BL21(DE3) which are lysogenic for $\lambda$-DE3.

In the first step, I have tried to compare the classical expression conditions in LB media with $1 \mathrm{mM}$ IPTG induction at $37^{\circ} \mathrm{C}$ for a few hours - in my experiments, I used 4 hours - with expression in minimal auto-inducing media (MD5052 [193], overnight at $18^{\circ} \mathrm{C}$. Figure 4.1, A shows a western blot of the comparison of the samples collected from these two conditions. For each condition, samples for the whole cell lysate, soluble fraction and soluble fraction enriched on Glutathione Sepharose 4B beads were investigated. The antibody used recognizes the GST tag, hence all the N-terminal degradation fragments of the GST-ScAtg18 fusion protein are recognized. 


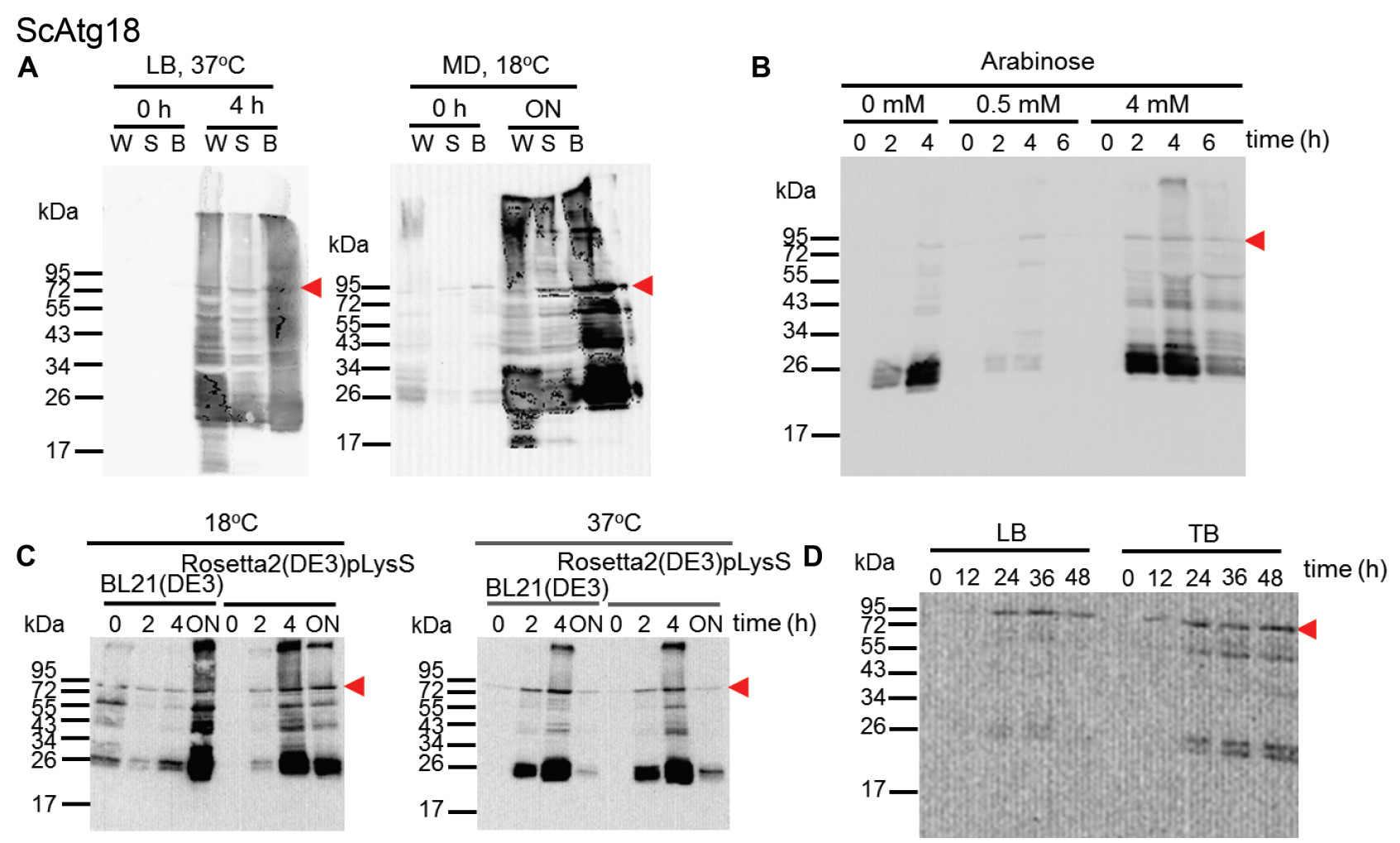

Figure 4.1: Expression optimization for ScAtg18 in E. coli.

Western blots representing the optimization steps for the expression of GST-ScAtg18. Detection is done with an anti-GST tag monoclonal antibody. Uninduced sample is represented as 0 hours. Red arrows indicate the where the GST-ScAtg18 full length protein would be expected. ON - overnight. (A) Expression of GST-ScAtg18 in E. coli BL21(DE3) cells in LB induced with $1 \mathrm{mM}$ IPTG at $37^{\circ} \mathrm{C}$ for 4 hours compared to minimal auto-inducing media (MD5052) in an overnight expression at $18^{\circ} \mathrm{C}$. W - whole cell lysate; $\mathrm{S}$ soluble fraction; B - soluble fraction enriched on Glutathione Sepharose 4B beads; MD - MD5052 (B) Coexpression of GST-ScAtg18 in BL21(DE3) with DnaJ, DnaK and GrpE chaperones. The effect of different concentrations of arabinose used for induction is investigated. For the 6 hours time point, cells are treated with $200 \mathrm{mM}$ Chloroamphenicol at $4 \mathrm{~h}$ after induction, in order to stop translation and allow 2 hours of folding of the translated peptides. Samples investigated are soluble fractions enriched on Glutathione Sepharose 4B beads. (C) Comparison of expression in E. coli BL21(DE3) cell and in E. coli Rosetta2(DE3)pLysS cells optimized for expression of proteins with rare codons. Protein expression at $18^{\circ} \mathrm{C}$ and $37^{\circ} \mathrm{C}$ is compared. Samples investigated are soluble fractions enriched on Glutathione Sepharose 4B beads. (D) Expression of GST-ScAtg18 in E. coli ArcticExpress(DE3) cells. LB and TB expression media are compared over a 48 hours time course. Samples investigated are soluble fractions enriched on Glutathione Sepharose 4B beads.

The expected full length size of the protein is approximately $80 \mathrm{kDa}$. For the uninduced sample, in the case of the LB media, a sample is harvested before the addition of the IPTG, while for the MD5052 media a sample is harvested when the $\mathrm{OD}_{600}$ reaches a value of approximatively 0.5 . It can be seen that in the case of all the samples a considerable amount of degradation products are obtained and there is little or no difference between the two conditions initially investigated. Overall, very little soluble full length GST-ScAtg18 is observed in the conditions tested in this step.

For the next optimization step, the influence of co-expression with protein chaperones was studied. Bacterial proteins have an average size of approximatively $30 \mathrm{kDa}$, while the human protein have an average size of $50 \mathrm{kDa}$ [194]. Comparative studies show that the probability of soluble bacterial expression decreases with an increase in molecular weight. This increase in size is due to evolution towards multidomain proteins. In addition, the expression of $\beta$-strands rich proteins is especially difficult since, in bacteria the entire peptide must be synthesized before folding is initiated, while in eukaryotes, translation and folding happen simultaneously. This difference makes it difficult to produce long $\beta$-sheets containing peptides since the high potential for forming 
intermolecular hydrogen bonds would make these peptides prone to aggregation. This is why, protein chaperones are needed, i.e., to prevent peptides from aggregating before translation is terminated. Moreover, in the case of human WIPI2, it was reported that its peptides were found interacting with human DnaJ family proteins [32]. Considering that degradation products, such as the ones shown in Figure 4.1, A appear in the case of not correctly folded proteins, I hypothesized that chaperones might help in the recovery of higher amounts of full-length soluble ScAtg18.

\section{ScAtg18}

A

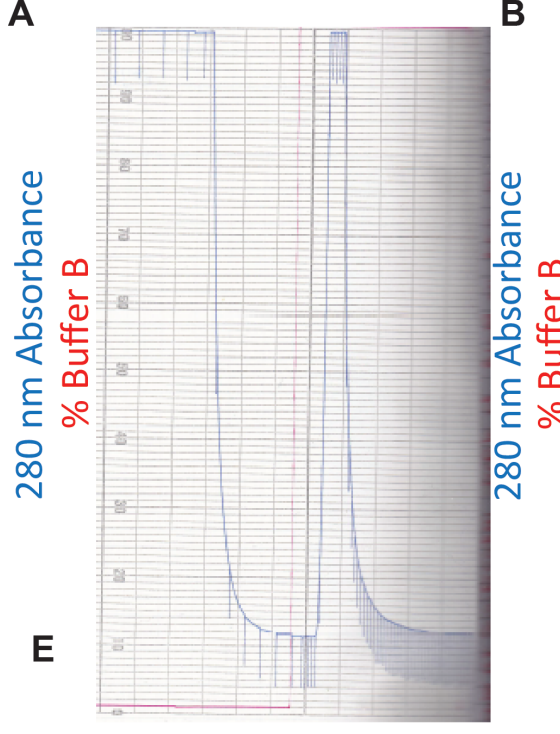

D

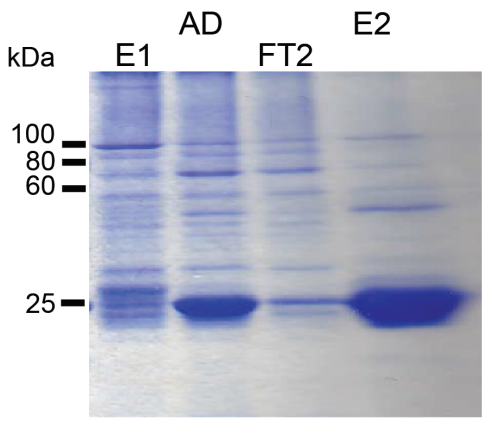

C

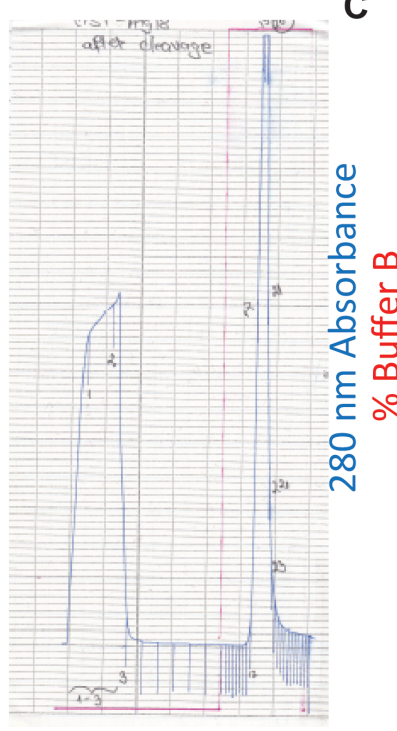

E

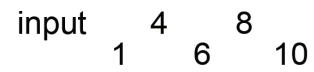

Figure 4.2: Purification of ScAtg18 from E. coli Rosetta2(DE3)pLysS.

Chromatogram of ScAtg 18 purified by (A) affinity chromatography using a GSTrap 4B column followed by overnight GST-tag removal using Prescission protease followed by another (B) affinity chromatography using a GSTrap 4B column. Next a gel filtration with HiLoad 16/60 Superdex S75 column was applied (C). The sample protein content can be followed on SDS-PAGE gels (D) for the affinity purifications, while the gel filtration samples are analyzed in (E). E - elution after the first affinity purification; AD - sample after dialysis and overnight thrombin cleavage; FT2 - flow through in the second affinity purification; E2 - elution after the second affinity purification; 1-20 - gel filtration elution fractions. The red arrow indicates PaAtg18.

In the case of the co-expression with chaperones optimization step, I have tried the pKJE7 plasmid (Takara, Clontech Laboratories, Inc.), containing the genes for DnaJ, DnaK and GrpE and pGro7 (Takara, Clontech Laboratories, Inc.), expressing GroES and GroEL. For both plasmids, the expression of chaperones is induced through arabinose. Moreover, the concentration of arabinose used for inducing the expression of the chaperons can control the amounts of chaperones produced. This is why three arabinose concentrations were investigated: 
$0 \mathrm{mM}$ - for the control of expression in the presence of no chaperones, $0.5 \mathrm{mM}$ and $4 \mathrm{mM}$ arabonose. Expression was done in LB media at $37^{\circ} \mathrm{C}$, while the induction of the GST-ScAtg18 expression was done with $1 \mathrm{mM}$ IPTG. Samples were taken before IPTG induction and at 2 and 4 hours after. For the 6 hours sample, at 4 hours after induction, $200 \mathrm{mM}$ Chloramphenicol were added to stop translation and allow 2 hours of folding of the translated peptides with shaking at $37^{\circ} \mathrm{C}$. In the case of the pKJE7 plasmid, the soluble samples enriched on Glutathione 4B sepharose beads are shown in Figure 4.1, B as detected by the anti-GST tag antibody through Western blotting. It can be observed that an increasing amount of GST-ScAtg18 is obtained with an increase in chaperones induction as compared with the expression free of chaperones. However, more free GST (molecular weight of $28.1 \mathrm{kDa}$ ) is also produced. A similar result was obtained for the pGro7 plasmid.

In the next step, the usage of the codons in the wild type gene of ScAtg18 was analyzed and it was observed that six problematic CTA (Leu) codons are present. E. coli has low usage of this codon, while the t-RNA for this codon is not abundant. This is why, special bacterial strains were engineered such that they contain plasmids to help in the expression of proteins from genes containing rare codons. One of these strains is E. coli Rosetta2(DE3)pLysS. A comparison in the expression of GST-ScAtg18 is done for BL21(DE3) and Rosetta2(DE3)pLysS strains as shown in Figure 4.1, C. The Western blot analysis shows soluble samples enriched on Glutathione 4B sepharose beads. The expression condition used was LB media with $1 \mathrm{mM}$ IPTG induction at $18^{\circ} \mathrm{C}$ and $37^{\circ} \mathrm{C}$. It appears that at low temperatures, more protein is produced and that the use of the E. coli Rosetta2(DE3)pLysS strain decreases the smear appearance given by the numerous GST-ScAtg18 degradation products. As a consequence, beside the full-length protein and the free GST protein, only two prominent degradation products can be observed, at approximatively 45 and $60 \mathrm{kDa}$.

The experiments performed until now show that low temperatures and chaperones improve folding. These two conditions exclude each other since common bacterial chaperones are active above $30^{\circ} \mathrm{C}$. This is why the E. coli ArcticExpress(DE3) bacterial strain was engineered to express Cpn10 and Cpn60 chaperonins from $O$. antarctica. These chaperonins are homologs of the E. coli GroEL and GroES chaperones, respectively. Cpn10 and Cpn60 show high protein refolding activities at temperatures of $4-12^{\circ} \mathrm{C}$. The expression of GST-ScAtg8 was probed in E. coli ArcticExpress(DE3) cells at $10^{\circ} \mathrm{C}$ for 48 hours, with samples taken every 12 hours. Soluble samples enriched on Glutathione 4B sepharose beads were compared in LB and TB media expression after the induction with $1 \mathrm{mM}$ IPTG as seen in Figure 4.1, D. There was not a significant difference between expression in TB when compared to the LB media. Even though the expression was low, it yielded cleaner sample, i.e., most of the protein produced was full length protein or free GST with a ratio between these two being more favorable to the full-length peptide as compared to all the other conditions tested before.

The purification of ScAtg18 was attempted in E. coli Rosetta2(DE3)pLysS cells grown in TB media with $1 \mathrm{mM}$ overnight IPTG induction at $18^{\circ} \mathrm{C}$. The expression scale was $6.6 \mathrm{~L}$. Cells were harvested and opened, then, the cell lysate loaded on a $5 \mathrm{~mL}$ GSTrap 4B column. The GST-fused protein was eluted with $20 \mathrm{mM}$ glutathione. The purification chromatogram for this step can be observed in Figure 4.2, A. The eluted protein was dialyzed overnight for glutathione and GST-tag removal using $0.02 \mathrm{U} / \mu \mathrm{L}$ Prescission protease. This was followed by another affinity chromatography using a GSTrap 4B column as shown in Figure 4.2, B. Next, the flow through sample was concentrated and loaded to a gel filtration HiLoad 16/60 Superdex S75 column. The chromatogram of this step can be seen in Figure 4.2, C. The sample protein content can be followed on SDSPAGE gels presented in Figure 4.2, D and E. For the affinity purifications, it can be observed that a lot more free GST than full-length fusion protein is obtained. Moreover, a high amount of other peptide fragments than 
the full-length GST-ScAtg18 protein are also recovered. After the second affinity purification, most of the free GST protein is removed. The gel filtration shows that most of the sample protein content elutes in the void volume, The SDS-PAGE analysis of the elution fractions identify fractions 4 to 6 as containing ScAtg18. The total protein yield is $1.2 \mathrm{mg}$ or $0.18 \mathrm{mg} / \mathrm{L}$ expression culture.

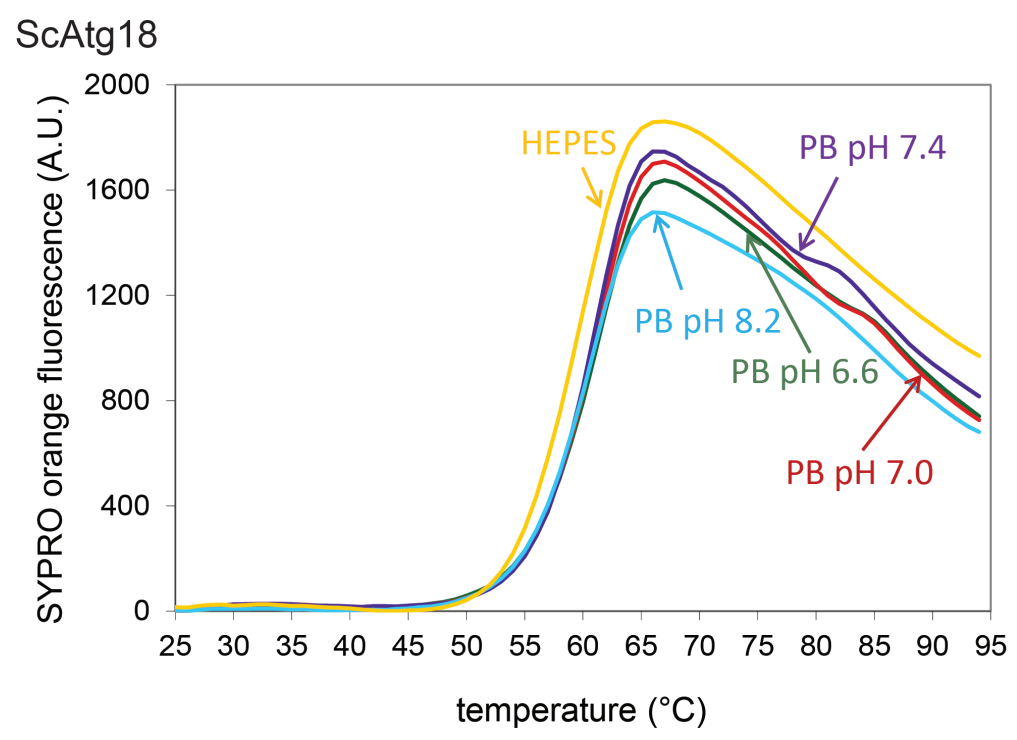

Figure 4.3: Stability analysis of ScAtg18 in several buffers.

Thermofluor experiments for ScAtg18 using the pHat buffer screen. Sypro Orange fluorescence was monitored and dequenching indicates unfolding of the proteins. Temperature was shifted from $25^{\circ} \mathrm{C}$ to $95^{\circ} \mathrm{C}$. In the conditions presented, an increase in protein stability was observed in comparison to the protein purification buffer. $30 \mathrm{mM}$ HEPES, pH 7.0, represented in yellow. PB - $100 \mathrm{mM}$ phosphate buffer.

The elution fraction 6 from the gel filtration was used for a thermofluor experiment [154]. This is a highthroughput stability assay in a 96-well format. Sypro Orange is a dye that increases its fluorescence by binding to the hydrophobic exposed protein patches. When the temperature of the sample is increased and the protein starts slowly unfolding, the Sypro orange fluoresce increases, while in later steps when peptides aggregate together, the dye detaches from the hydrophobic patches leading to a decrease in fluorescence. The point of inflection during the increase in fluorescence gives the melting temperature. A more stable condition can be easily observed from having a slope that intersects the $\mathrm{x}$-axis more to the right side of the graph. Once purified, a buffer stability assay was performed on ScAtg18 using the pHat screen.

The stability assay on ScAtg18 (Figure 4.3) has revealed that only phosphate buffers of any pH can increase the buffer stability of ScAtg18 over the HEPES, pH 7.0 buffer in which the initial purification was done.

\subsubsection{Expression of S. cerevisiae Atg21}

I used the previously reported expression vector construct from the Thumm lab [94]. As in the case of ScAtg18, the plasmid expresses a ScAtg21 a fusion with a N-terminal GST tag. The tag can be cut using thrombin protease. The gene sequence encoded was amplified from S. cerevisiae, hence it contained the wild type gene sequence. The promoter used for transcription is the tac promoter. The optimization steps are the same as done for ScAtg18. 


\section{ScAtg21}

A

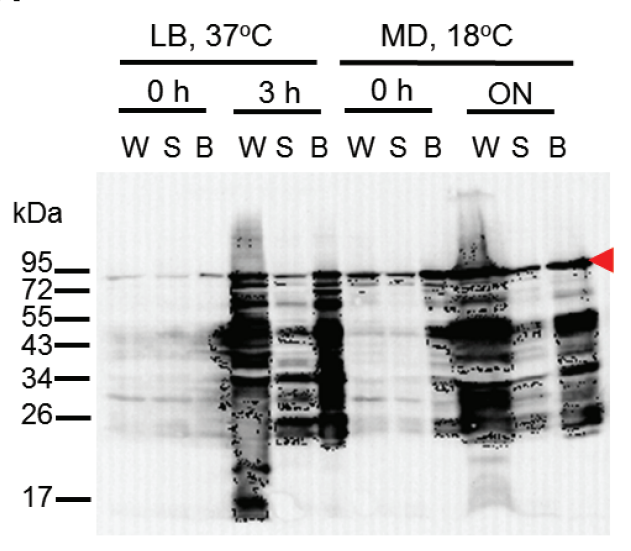

B

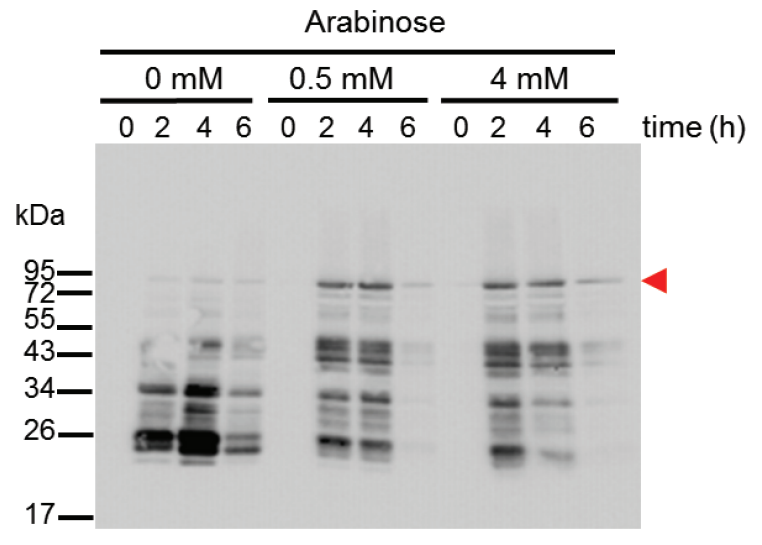

C

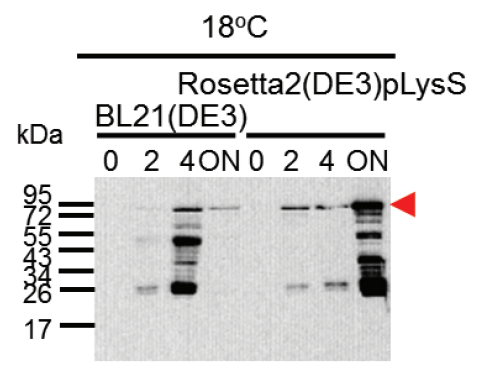

$37^{\circ} \mathrm{C}$

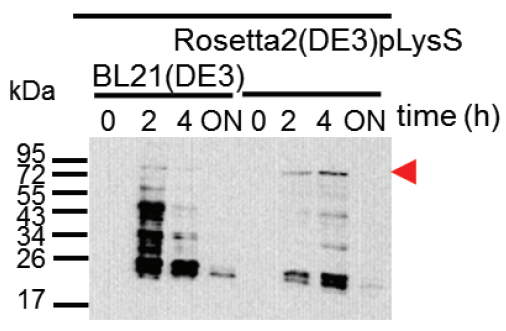

D 
In the first step, I have tried to compare the classical expression conditions in LB media with $1 \mathrm{mM}$ IPTG induction at $37^{\circ} \mathrm{C}$ for a few hours - in my experiments, I used 4 hours - with expression in minimal autoinducing media (MD5052 [193], overnight at $18^{\circ} \mathrm{C}$. Figure 4.4, A shows a western blot of the comparison of the samples collected from these two conditions. The expected full length size of the protein is approximately $80 \mathrm{kDa}$. Similar degradation products as in the case of ScAtg18 can be observed.

Next, I studied the influence of co-expression with protein chaperones. As before, pKJE7 and pGro7 plasmids were used for protein chaperones co-expression and the influence of arabinose concentration for induction was investigated. The expression conditions and sampling was done as before. In the case of the pKJE7 plasmid, the soluble samples enriched on Glutathione 4B sepharose beads are shown in Figure 4.4, B. It can be observed that an increasing amount of full lenght GST-ScAtg21 is obtained with an increase in chaperones induction as compared with the no chaperones expression where mainly small degradation products are detected. The translation block with Chloramphenicol induced overall protein degradation. The pGro7 plasmid yielded similar results.

When the usage of the codons in the wild type gene of ScAtg21 was analyzed, it was noticed that six problematic CTA (Leu) codons are present. This is why, next a comparison in the expression of GST-ScAtg21 is done for E. coli BL21(DE3) and E. coli Rosetta2(DE3)pLysS strains as shown in Figure 4.4, C. The Western blot shows soluble samples enriched on Glutathione 4B sepharose beads. The expression condition used was TB media with $1 \mathrm{mM}$ IPTG induction at $18^{\circ} \mathrm{C}$ and $37^{\circ} \mathrm{C}$. It appears that at low temperatures, more full-lenght protein is produced. E. coli Rosetta2(DE3)pLysS cells express more full-length protein, however there are also more small degradation products observed. Beside the full-length and free GST protein, a band for a degradation product of approximatively $50 \mathrm{kDa}$ can be clearly distinguished from the other smaller fragments.

In a last optimization step, E. coli ArcticExpress(DE3) bacterial strain was probed as a potential expression host. The same expression conditions were used as in the case of GST-ScAtg18. Soluble samples enriched on Glutathione 4B sepharose beads were investigated as shown in Figure 4.4, D. The results were similar to the ScAtg18 ones, namely a low, but cleaner full-length expression product with higher fusion protein to free GST ratio.

As done for ScAtg18, the purification of ScAtg21 was attempted. Similarly, E. coli Rosetta2(DE3)pLysS was used together with TB media with $1 \mathrm{mM}$ overnight IPTG induction at $18^{\circ} \mathrm{C}$. However, in the case of ScAtg21, ever a lower protein quantity was obtained. The yield was much lower than $1 \mathrm{mg}$ and could not be used for a thermofluor buffer stability experiment.

\subsubsection{Expression and purification of $P$. angusta Atg18}

Since the expression and purification of S. cerevisiae Atg18 was not successful, I have followed on the work of Dr. Ricarda Busse [192] and I have further optimized the purification protocol for PaAtg18. Initially, the previously established protocol was tried for the high-amounts production of PaAtg18. In short, this protocol involved an affinity chromatography step using a HisTrap FF column followed by a dialysis from HEPES, $\mathrm{pH}$ 7.0 to Na-lactate buffer, $\mathrm{pH}$ 4.0. Finally, a gel filtration step with HiLoad 16/60 Superdex S75 column was performed. However, during the dialysis and concentration steps before the gel filtration purification, a lot of protein precipitated. This is why, after the gel filtration only a small amount of PaAtg18 could be obtained, as 


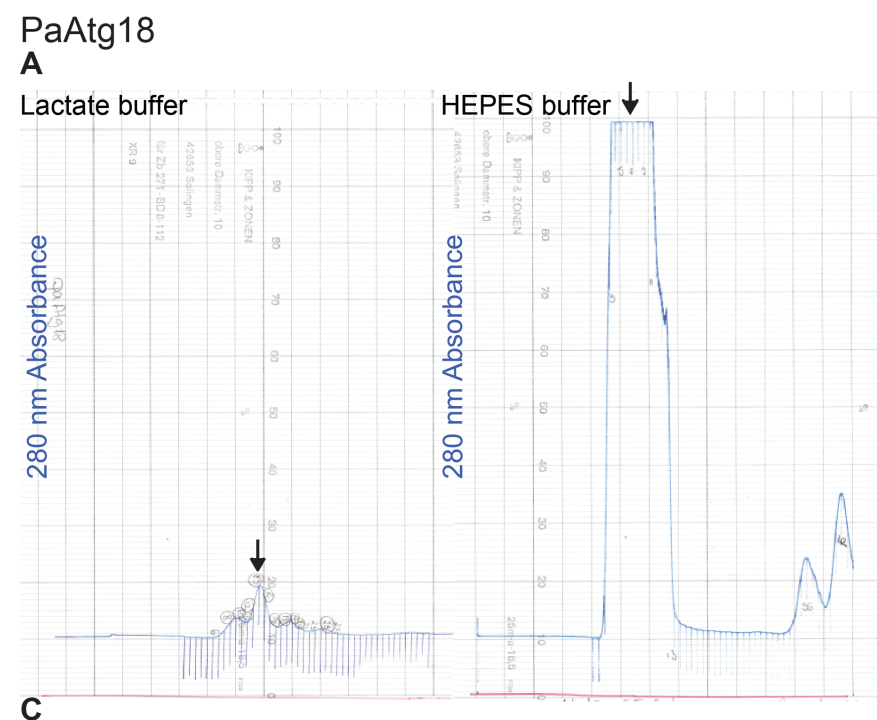

B
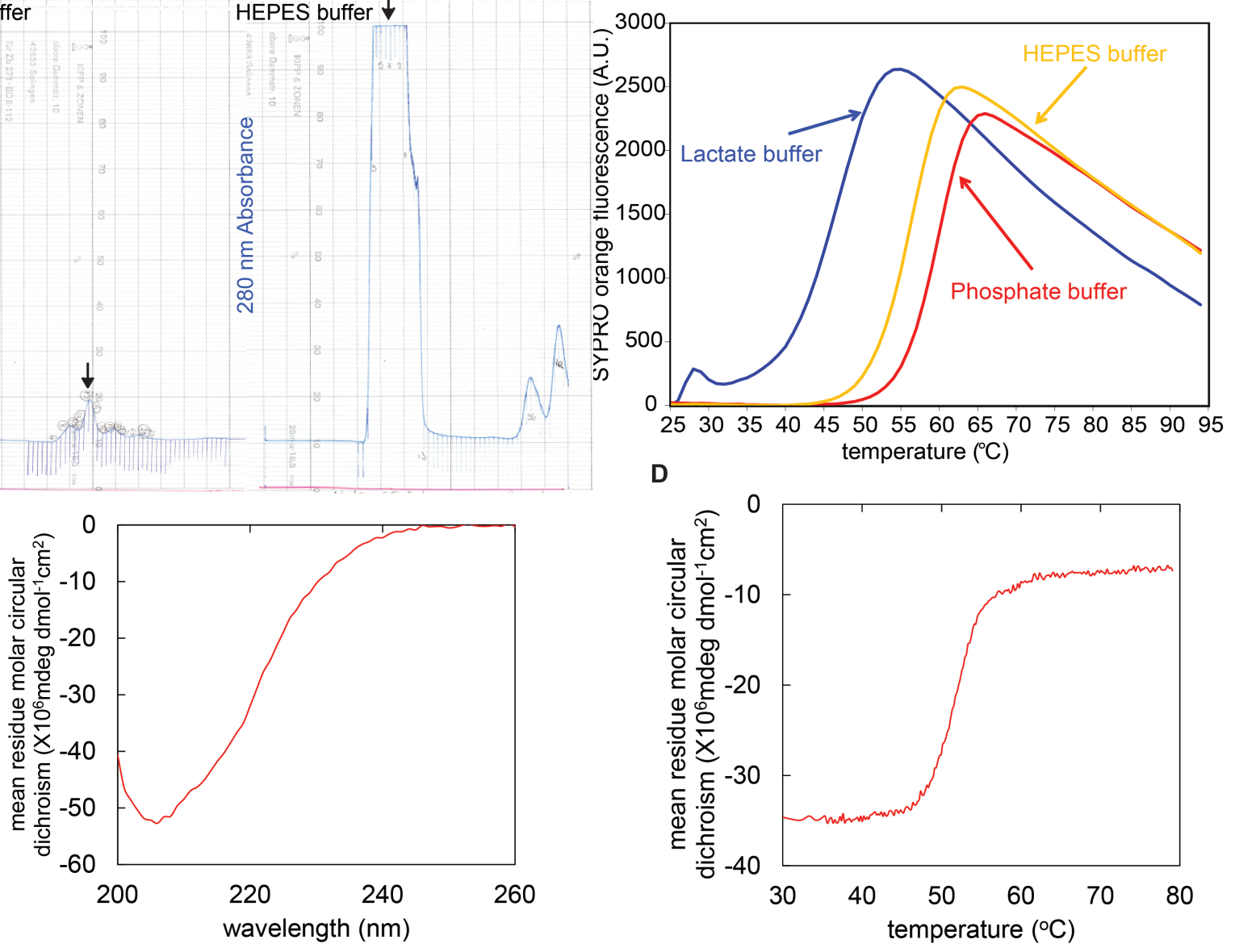

Figure 4.5: Buffer optimization for the purification of PaAtg18 and its stability analysis.

(A) Comparison between the chromatograms of gel filtration with HiLoad 16/60 Superdex S75 column step for the purification of PaAtg18 in Na-lactate buffer, pH 4.0 and HEPES buffer, pH 7.0. Black arrow shows the PaAtg18 elution peak. (B) Thermofluor stability assay for PaAtg18 in Na-lactate ( $\mathrm{pH} 4.0$ ), HEPES ( $\mathrm{pH}$ 7.0) and phosphate buffer (pH 5.8). Sypro Orange fluorescence was monitored and dequenching indicates unfolding of the proteins. Temperature was shifted from $25^{\circ} \mathrm{C}$ to $95^{\circ} \mathrm{C}$. (C) $\mathrm{CD}$ spectrum of PaAtg18 from $260 \mathrm{~nm}$ to $200 \mathrm{~nm}$. (D) Melting curve for PaAtg 18 was taken at $216 \mathrm{~nm}$ from $30^{\circ} \mathrm{C}$ to $80^{\circ} \mathrm{C}$. 
shown by the low height of the elution peak for PaAtg18 on the left panel in Figure 4.5, A. In contract, when using HEPES, pH 7.0 buffer, a high amount of PaAtg18 could be observed.

In order to investigate the protein stability in different buffers, a thermofluor experiment was performed (Figure 4.5 B). It can be observed that the Na-lactate, $\mathrm{pH} 4.0$ buffer is a less stable condition than the HEPES, pH 7.0 buffer. Moreover, as in the case for ScAtg18, phosphate buffer stabilizes the protein the most.

Once the protein was purified, its folding was checked by measuring a CD spectrum from $260 \mathrm{~nm}$ down to $200 \mathrm{~nm}$ (Figure 4.5. C). Interestingly, a minimum in the spectrum was observed below $210 \mathrm{~nm}$, even though for a protein rich in $\beta$-strands would be expected a minimum at $216 \mathrm{~nm}$. This is why a melting curve from $30^{\circ} \mathrm{C}$ to $80^{\circ} \mathrm{C}$ was recorded at $216 \mathrm{~nm}$ Figure 4.5, D). An unfolding curve, with a melting temperature of $(53.41 \pm 0.06)^{\circ} \mathrm{C}$ was observed, showing that the initial protein sample had a folding state that changed.

Using HEPES, pH 7.0 buffer. PaAtg18 was purified in high amount for both crystallization and biochemical investigations. The yield per purification varied in a range of $10-20 \mathrm{mg} / \mathrm{L}$ expression culture. Using the same protocol, single cysteine mutants were purified for experiments for investigating the orientation of PaAtg18 towards the membrane it binds to. Figure 4.6 shows

\subsubsection{Expression and purification of $K$. lactis Atg21}

The expression and purification of $S$. cerevisiae Atg21 was not successful. This is why, I have further optimized the protocol developed by Dr. Ricarda Busse [192] for the expression and purification of KlAtg21. The initial protocol recommended the use of MES buffer, $\mathrm{pH}$ 5.8. Having difficulties with the stability of the protein in this buffer, based on the experience with PaAtg18, I have switched to HEPES buffer, pH 7.0. Thanks to its $\mathrm{N}$-terminal His-tag, KlAtg21 can be easily purified in a two step purification: affinity chromatography using a HisTrap FF column followed by a gel filtration step with HiLoad 16/60 Superdex S75 column. The protein content after each purification step can be followed on SDS-PAGE (Figure 4.7). Panel A shows the results after the first change in the original protocol was implemented, namely using HEPES buffer, $\mathrm{pH} 7.0$ during the gel filtration step. On an SDS-PAGE gel, KlAtg21 is expected to run approximatively around $45 \mathrm{kDa}$. The full length KlAtg21 can be observed in small amounts, while most of the expression and purification product is a degradation product of approximatively $35 \mathrm{kDa}$. This is why, next, I have tested the expression under milder inducing conditions by switching the expression media from ZYM5052 to MD5052. The purification results are shown in Figure 4.7 B. As a result of the MD5052 expression, the full length protein to degradation product ratio increased.

Once KlAtg21 was purified, before using it in biochemical experiments, its folding and stability were tested. A CD spectrum from $260 \mathrm{~nm}$ to $200 \mathrm{~nm}$ was measured, while a melting curve was taken at $216 \mathrm{~nm}$ from $30^{\circ} \mathrm{C}$ to $80^{\circ} \mathrm{C}$, as shown in Figure 4.7 $\mathrm{C}$ and D. As expected for a $\beta$-sheet-rich protein, K1Atg21 has a spectrum minimum at $216 \mathrm{~nm}$ showing a well folded protein. Its melting curve revealed that the melting temperature of $\mathrm{K} 1 \mathrm{Atg} 21$ is $(56.5 \pm 0.3)^{\circ} \mathrm{C}$

\subsubsection{Expression and purification of S.cerevisiae Hsv2}

In order to perform the biochemical analysis of ScHsv2 and its mutants, the protein had to be expressed and purified. The protocol established by Dr. Ricarda Busse [192] was used without any modifications. In short, the purification consists of an affinity step, followed by overnight dialysis with GST-tag removal by 


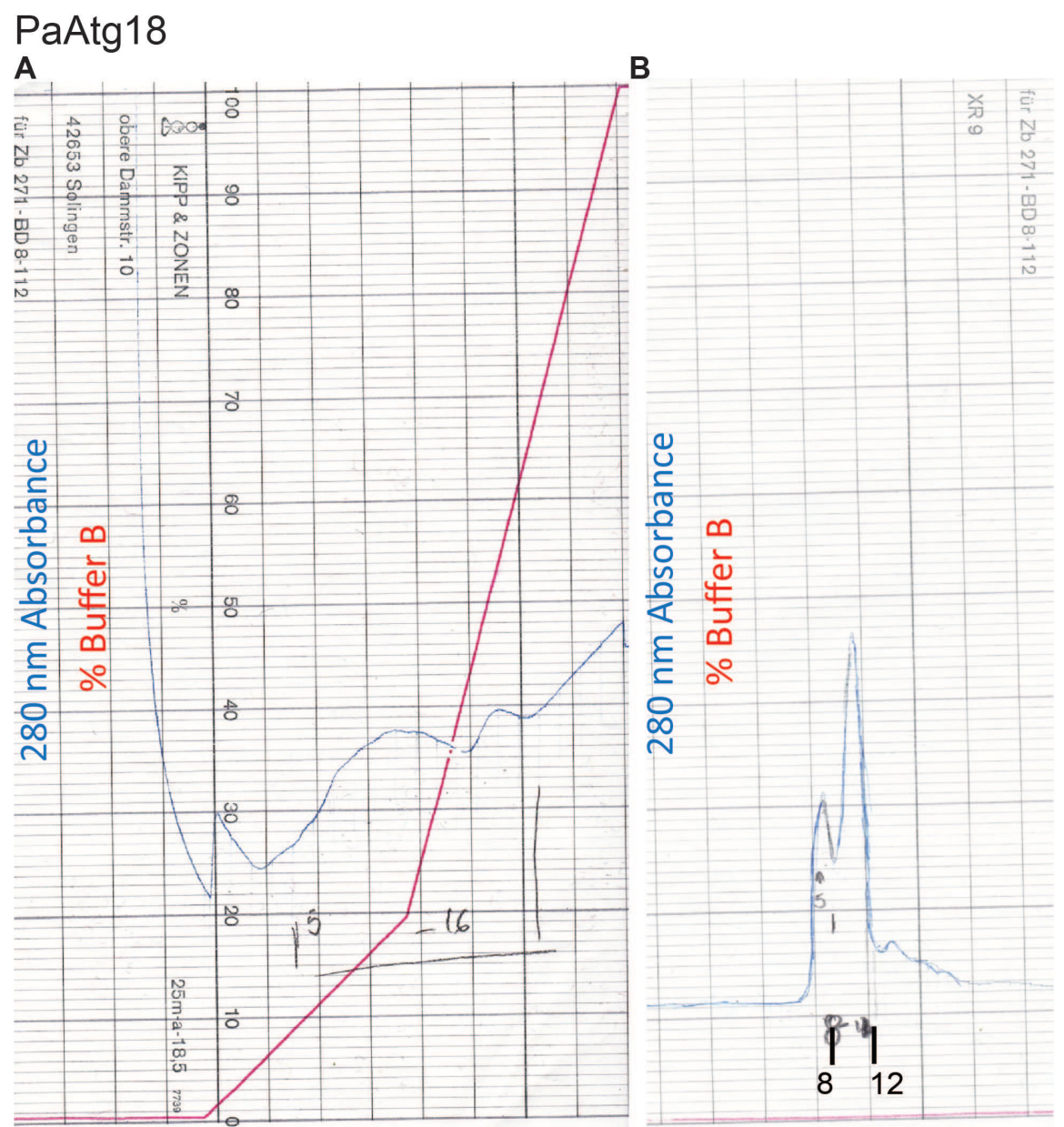

C

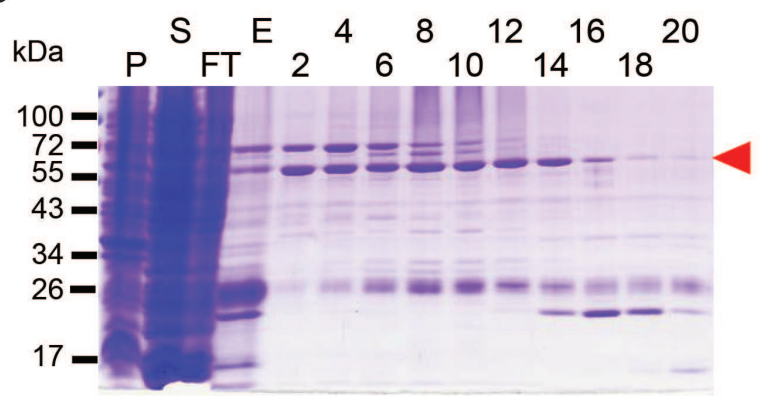

Figure 4.6: Purification of PaAtg18 no cysteines, S81C mutant from $E$. coli BL21(DE3)

Chromatograms of PaAtg18 no cysteines, S81C mutant purified by (A) affinity chromatography using a HisTrap FF column followed by (B) gel filtration with HiLoad 16/60 Superdex S75 column. Fractions 8-12 containing PaAtg18 are marked. (C) The protein content during is purification step can be followed on an SDS-PAGE. P - pellet/insoluble fraction; S - soluble fraction; E - affinity purification elution; 2-20 - gel filtration elution fractions. Red arrow indicated PaAtg18. 

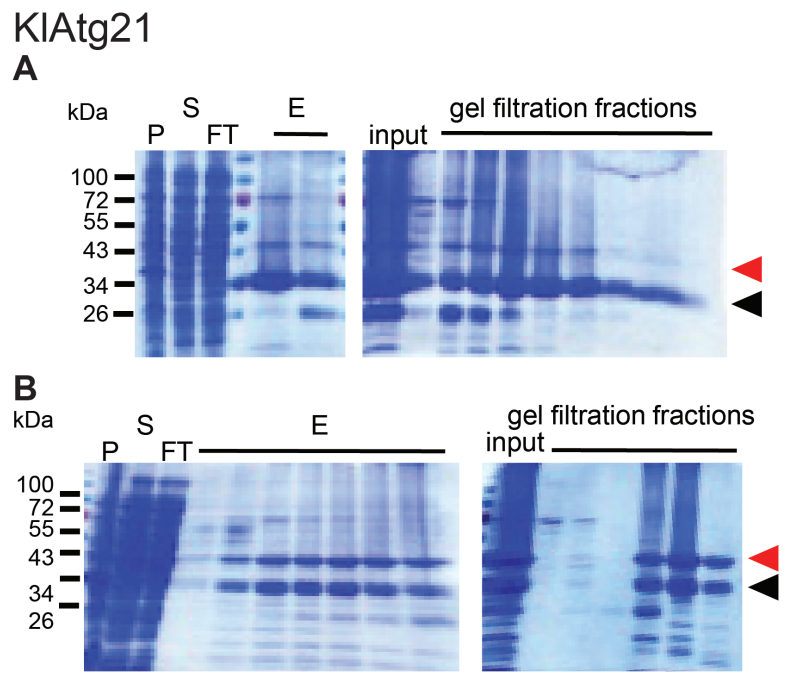

C

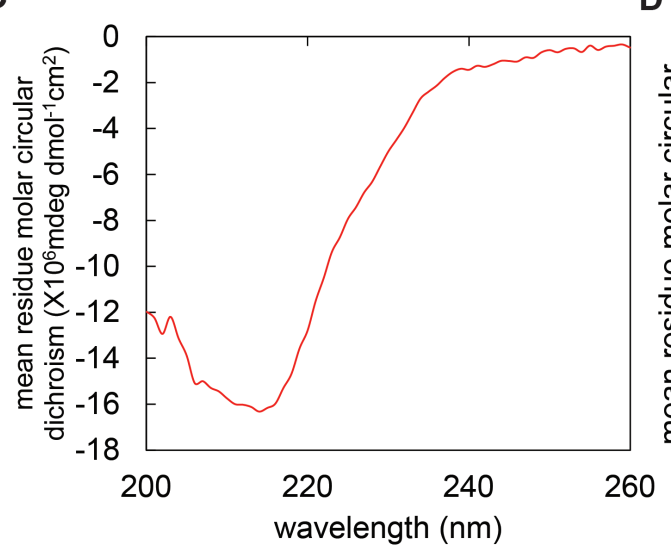

D

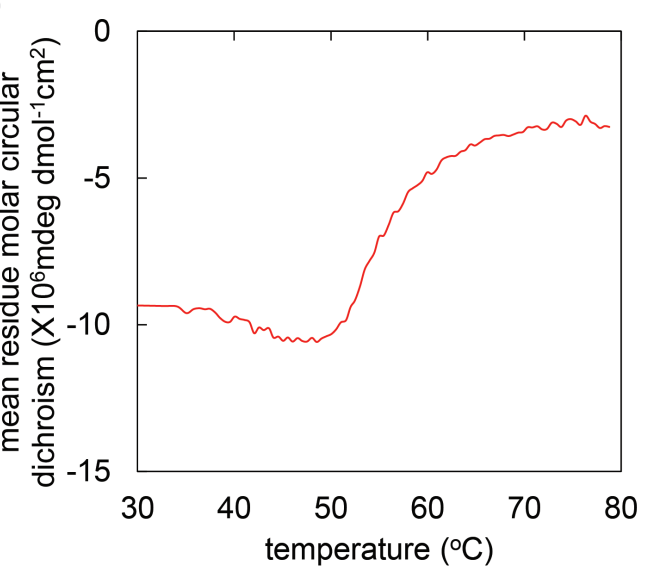

Figure 4.7: Purification of KlAtg21 from E. coli

Purification of K1Atg21 expressed in ZYM5052 (A) compared to MD5052 (B) autoinducing media. An affinity purification step was followed by a gel filtration purification. P - pellet/insoluble fraction; S - soluble fraction; FT - flow through; E - affinity column elution fractions. The red arrow indicates full length KlAtg21, while the black arrow indicates a degradation product. (C) CD spectrum of $\mathrm{K} 1 \mathrm{Atg} 21$ from $260 \mathrm{~nm}$ to $200 \mathrm{~nm}$. (D) Melting curve for KlAtg21 was taken at $216 \mathrm{~nm}$ from $30^{\circ} \mathrm{C}$ to $80^{\circ} \mathrm{C}$. 
thrombin. Next, another affinity purification step was performed. Finally, the flow through of the previous step was loaded on a gel filtration column. The protein sample was analyzed after each step on SDS-PAGE. Figure 4.8 shows the resulting SDS-PAGE gels after the wild type ScHsv2 purification. One observes that even after the gel filtration, it is difficult to completely remove the GST-fusion protein and free GST from ScHsv2. ScHsv2(R264A) and ScHsv2(H294A) single binding site mutants were purified using the same methodology and yielded similar results.

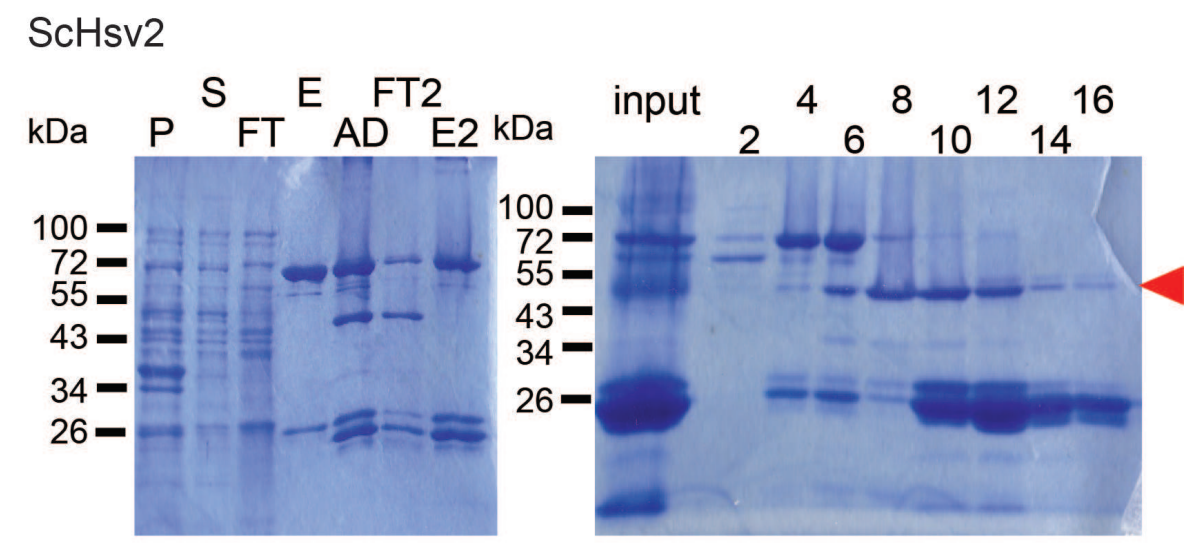

Figure 4.8: Purification of ScHsv2 from E. coli

An affinity purification step was followed by overnight dialysis with GST-tag removal by thrombin. Another affinity purification step and a gel filtration purification were further performed. P - pellet/insoluble fraction; S - soluble fraction; FT - flow through; E - first affinity purification elution purification; AD - sample after dialysis/GST-tag cleavage; FT2 - flow through after the second affinity purification; E2 - elution fraction after the second affinity purification; input - input for the gel filtration purification step; 2-16 - gel filtration elution fractions. The red arrow indicates full length ScHsv2.

Table 4.1: Melting temperatures of ScHsv2 wild type and mutants as measured through CD.

\begin{tabular}{ccc}
\hline & $\mathbf{T}_{m}\left({ }^{o} \mathbf{C}\right)$ & Fitting error $\left({ }^{o} \mathbf{C}\right)$ \\
\hline wild type & 54.58 & 0.05 \\
R264A & 53.63 & 0.05 \\
H294A & 54.20 & 0.05 \\
\hline
\end{tabular}

Once the wild type ScHsv2 and its mutants were purified, their folding and stability were tested. CD spectra from $260 \mathrm{~nm}$ to $200 \mathrm{~nm}$ and melting curves taken at $216 \mathrm{~nm}$ from $30^{\circ} \mathrm{C}$ to $80^{\circ} \mathrm{C}$ were measured, as shown in Figure 4.9. ScHsv2 and its mutants have a spectrum minimum around $210 \mathrm{~nm}$, but their melting curves revealed that there is a three-dimensional structure that slowly unfolds. The melting temperatures determined are summarized in Table 4.1 The ScHsv2 mutants have similar melting temperatures to the wild type protein.

\subsubsection{Expression and purification of C. thermophilum Atg18}

Since attempts to express and purify ScAtg18 were not successful, Chaetomium thermophilum Atg18 (CtAtg18) was tested instead. C. thermophilum is a thermophilic fungus that was used successfully for crystallization due it its likely more stable proteins [195]. CtAtg18 has 32.8\% identity with ScAtg18.

The CtAtg18 synthetic gene was ordered after codon optimization for bacterial and insect cell expression was done. Next, taking into account previous difficulties for the expression of ScAtg18 in bacteria, together 


\section{ScHsv2}

A
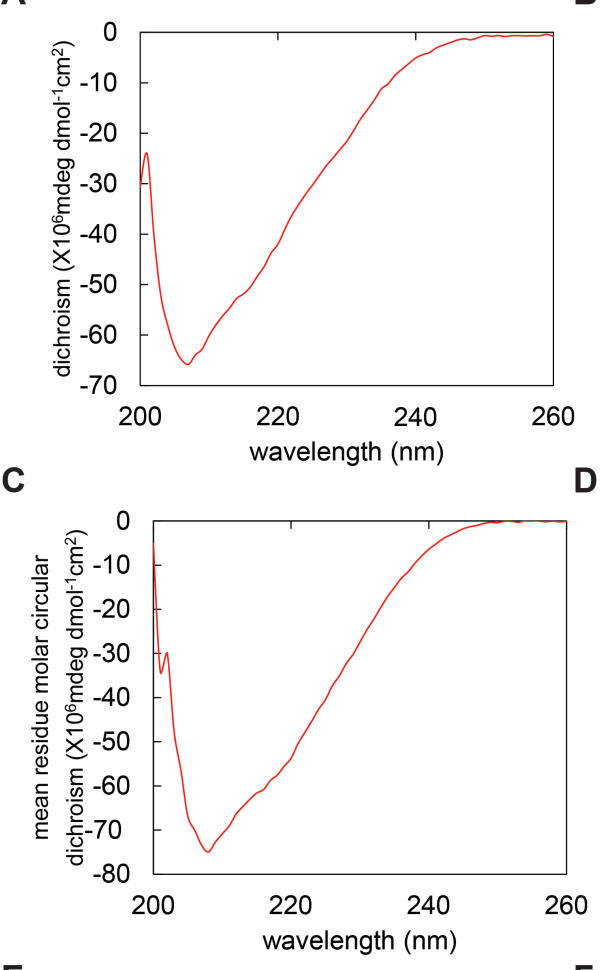

E

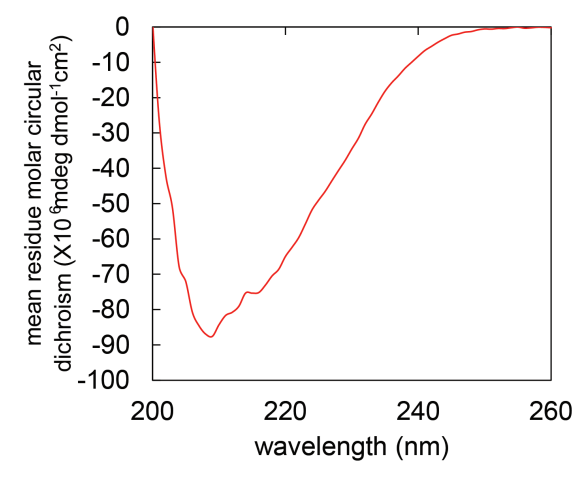

B

$\mathbf{F}$
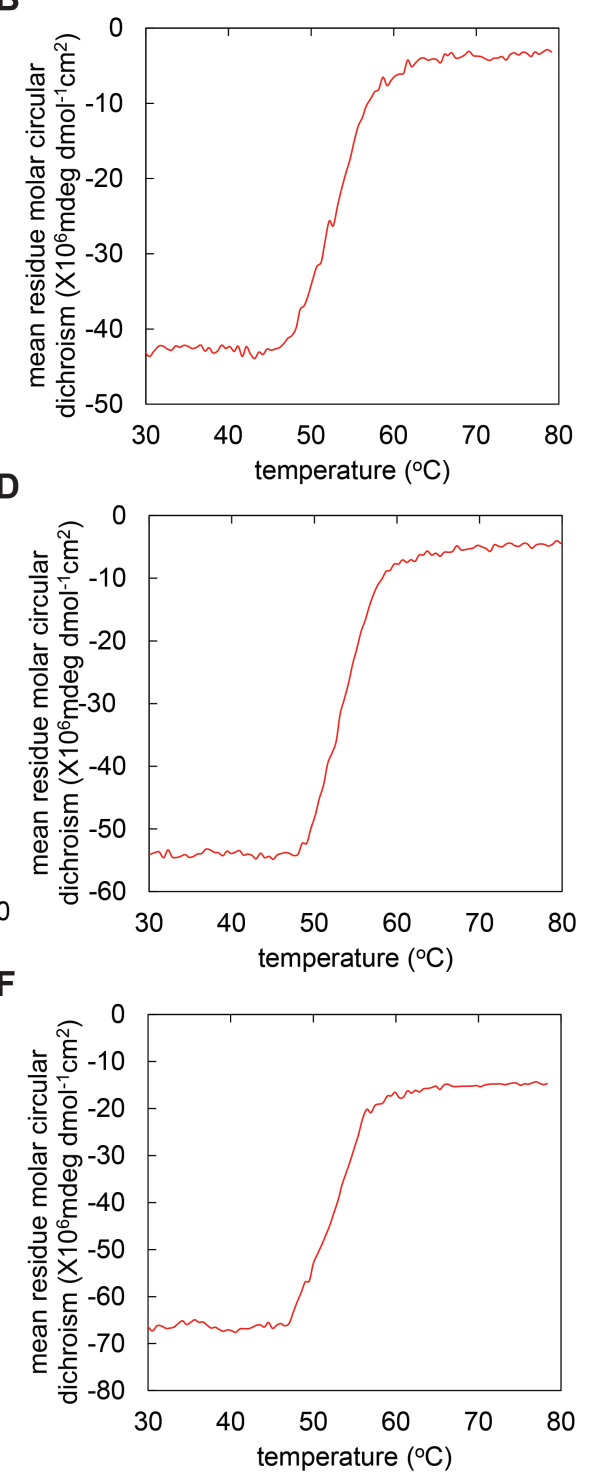

Figure 4.9: Stability of wild type ScHsv2 and mutants as measured through CD.

$\mathrm{CD}$ spectra from $260 \mathrm{~nm}$ to $200 \mathrm{~nm}$ and melting curves taken at $216 \mathrm{~nm}$ from $30^{\circ} \mathrm{C}$ to $80^{\circ} \mathrm{C}$ for wild type ScHsv2 (A), (B); ScHsv2(R264A) (C), (D) and ScHsv2(H294A) (E), (F) 


\section{CtAtg18}

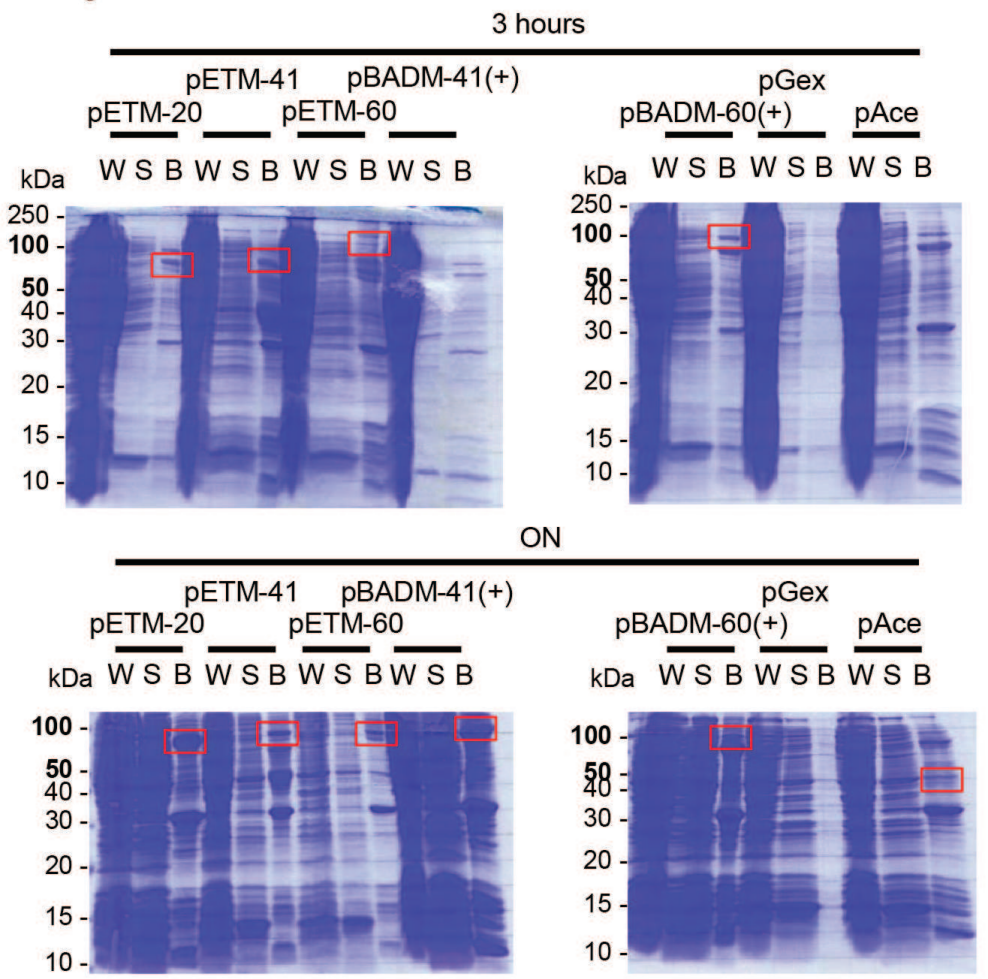

Figure 4.10: Expression tests for CtAtg18 in different expression vectors.

CtAtg18 gene was cloned in several vectors and its expression was tested in E. coli Bl21(DE3). Samples were taken after 3 hours of induction and after overnight expression in TB media induced with $0.1 \mathrm{mM}$ IPTG for tac or T7 promoters or $1 \mathrm{mg} / \mathrm{mL}$ arabinose at $22^{\circ} \mathrm{C}$. The protein product is marked in a red rectangle for the beads enriched samples. ON - samples harvested after overnight expression. W - whole cell lysate; S - soluble fraction; B - soluble fraction enriched on affinity beads; ON - overnight. 
with my Master student, Oleksandr Yagensky, I decided to clone CtAtg18 into several expression vectors with different properties (Table 4.2). Different promoters: T7, tac and $\operatorname{araBAD}$, and different affinity and fusion tags: His, GST (glutathione S-transferase), MBP (maltose binding protein), TrxA (thioredoxin-1), NusA (N utilization substance $\mathbf{A}$ ). The promoters were chosen such that either a maximum protein yield is obtained, as it is generally the case for the $T 7$ promoter or, in case the protein is toxic for the cells, a minimum basal expression is ensured, as in the case of the $\operatorname{araBAD}$ promoter [194]. These particular fusion tags were chosen because MBP, TrxA and NusA were reported to improve protein folding [196].

Table 4.2: Summary of the expression vectors tested for the expression of CtAtg18. The properties of these vectors such as promoter and its inducer, affinity and fusion tags and cloning sites are listed together with the expected protein product size and the cloning sites used for cloning the CtAtg18 gene. MW - molecular weight.

\begin{tabular}{ccccccc}
\hline Vector & Promoter & Inducer & Affinity; fusion tag & Resistance & Cloning sites & MW (kDa) \\
\hline pAce1-N-His & T7 & IPTG & 6xHis & AmpR & NdeI, XhoI & 50.1 \\
pETM-20 & T7 & IPTG & 6xHis; TrxA & AmpR & NotI, NcoI & 62.3 \\
pETM-41 & T7 & IPTG & 6xHis; MBP & KanR & NotI, NcoI & 91 \\
pETM-60 & T7 & IPTG & 6xHis; NusA & KanR & NotI, NcoI & 105 \\
pBADM-41(+) & ARA & Arabinose & 6xHis; MBP & AmpR & XhoI, NcoI & 91 \\
pBADM-60(+) & ARA & Arabinose & 6xHis; NusA & AmpR & XhoI, NcoI & 105 \\
pGex-4T-1 & tac & IPTG & GST & AmpR & XhoI, BamHI & 74.2 \\
\hline
\end{tabular}

Protein expression was tested in all the constructs summarized in Table 4.2 in TB media in an overnight expression induced with $0.1 \mathrm{mM}$ IPTG or $1 \mathrm{mg} / \mathrm{L}$ arabinose at $22^{\circ} \mathrm{C}$. The results are shown in Figure 4.10. CtAtg18 was expressed in a soluble form in all conditions except when using the pGex-4T-1-ctAtg 18 vector.

pAce1-N-His-CtAtg18 construct was chosen for further optimization because of the possibility to recombine, using Cre recombinase, with the pDk vector. The pAce1-N-His and pDk vectors are part of the ACEMBL expression system developed for protein complex production [197]. At a later stage [6.1.2), the donor plasmid, pDk was used for cloning a C. thermophilum Atg2 domain. This would allow the expression and purification of the Atg2-Atg18 complex. Another expression test was performed to determine the optimum expression conditions for CtAtg18 in E. coli BL21(DE3). This time both auto-inducing media (MD5052 and ZYM5052) and media inducible by the addition of IPTG (LB and TB) were tested at $18^{\circ} \mathrm{C}$ and $37^{\circ} \mathrm{C}$. Samples were taken before induction with IPTG, for LB and TB, or in the case of auto-inducing media, when $\mathrm{OD}_{600}$ was approximatively 0.5. Further samples were taken at 3 hours after induction and after overnight expression. Whole cell extract, soluble fractions and $\mathrm{Ni}^{2+}$-sepharose enriched soluble fractions were analyzed on SDS-PAGE gels (Figure 4.11). A weak enrichment of a protein with a size of approximatively $60 \mathrm{kDa}$ was observed for MD5052 media. The expected size for CtAtg18 is $50 \mathrm{kDa}$.

Based on the SDS-PAGE analysis, we decided to express CtAtg 18 for a large scale purification overnight in MD5052 at $18^{\circ} \mathrm{C}$. Unfortunately, after the purification, when the purification product was sent for confirmation to mass spectrometry, it was confirmed that it is not the target protein, but a bacterial contaminant. 
CtAtg18
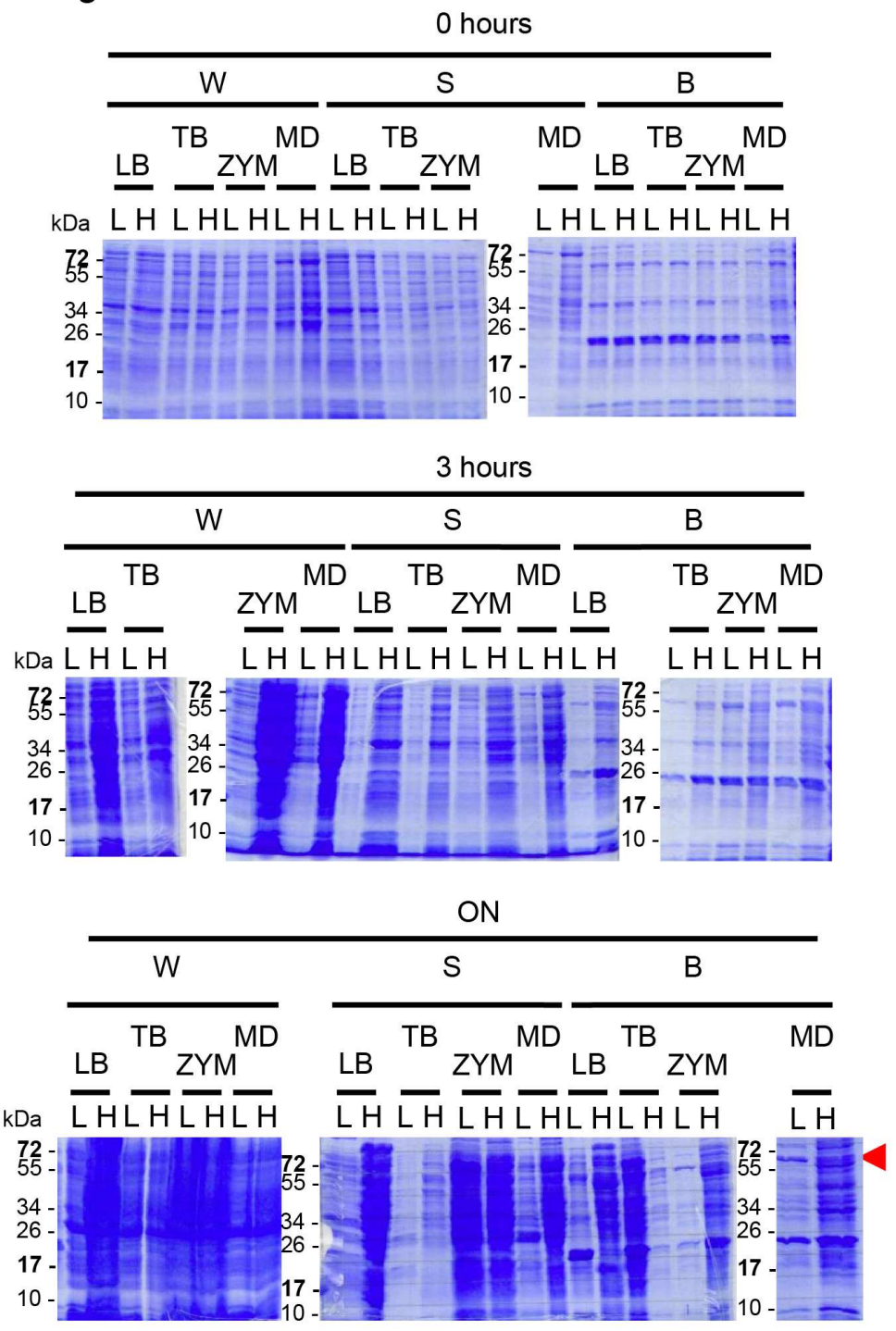

Figure 4.11: Expression tests CtAtg18 in pAce1-N-His expression vector in $E$. coli B121(DE3)

The expression of CtAtg18 from pAce1-N-His was tested in different conditions. Media tested was either LB or TB with $1 \mathrm{mM}$ IPTG induction or auto-inducing media, MD5052 (MD) or ZYM5052 (ZYM). Two temperatures were tested, $18^{\circ} \mathrm{C}(\mathrm{L})$ and $37^{\circ} \mathrm{C}(\mathrm{H})$. Samples were taken before induction and after 3 hours of induction and after overnight expression. The protein product is marked with a red arrow for the overnight, MD5052 expression media, $\mathrm{Ni}^{2+}$-sepharose enriched samples (bottom panel). ON - samples harvested after overnight expression. W - whole cell lysate; $\mathrm{S}$ - soluble fraction; B - soluble fraction enriched on $\mathrm{Ni}^{2+}$-sepharose beads; $\mathrm{ON}$ overnight. 


\subsection{ITC measurements of PROPPINs with PI3P and PI(3,5) $P_{2}$ containing li- posomes}

Having optimized protocols for the expression and purification of PaAtg18 and K1Atg21, together with the protocol previously established for the purification of ScHsv2 and its mutants [1, 192], I could undertake the biochemical characterization of the yeast PROPPIN paralogs. This section reports on the biochemical findings using isothermal titration calorimetry (ITC) to measure PROPPIN phosphoinositide binding.

All PROPPIN paralogs specifically bind PI3P and PI $(3,5) \mathrm{P}_{2}$, as shown through flotation assays with SUVs (small unilamellar vesicles) [192]. Using a protocol established in our lab [155], I used ITC to titrate PROPPINs into SUVs containing $2 \%$ of either $\mathrm{PI} 3 \mathrm{P}$ or $\mathrm{PI}(3,5) \mathrm{P}_{2}$. A summary of the thermodynamic parameters found is given in Table 4.3 .

Table 4.3: Summary of thermodynamic parameters as determined through ITC measurements for PROPPIN PI3P or PI( $(3,5) \mathrm{P}_{2}$ binding. $\mathrm{n}$ - number of measurements; $\mathrm{N}$ - binding stoichiometry. Errors reported are standard errors of the mean.

\begin{tabular}{cccccccc}
\hline & & $\mathbf{n}$ & $\mathbf{N}$ & $\begin{array}{c}\mathbf{K}_{d} \\
(\mu \mathbf{M})\end{array}$ & $\begin{array}{c}\Delta \mathbf{H} \\
(\mathbf{k J} / \mathbf{m o l})\end{array}$ & $\begin{array}{c}\mathbf{T} \Delta \mathbf{S} \\
(\mathbf{k J} / \mathbf{m o l})\end{array}$ & $\begin{array}{c}\Delta \mathbf{G} \\
(\mathbf{k J} / \mathbf{m o l})\end{array}$ \\
\hline \hline PaAtg18 & $\mathrm{PI3P}$ & 4 & $0.03 \pm 0.01$ & $0.29 \pm 0.09$ & $-101 \pm 23$ & $-63 \pm 24$ & $-37.8 \pm 1.1$ \\
& $\mathrm{PI}(3,5) \mathrm{P}_{2}$ & 8 & $0.50 \pm 0.02$ & $0.11 \pm 0.01$ & $-147 \pm 6$ & $-107 \pm 6$ & $-39.9 \pm 0.3$ \\
\hline KlAtg21 & $\mathrm{PI} 3 \mathrm{P}$ & 3 & $0.44 \pm 0.19$ & $0.86 \pm 0.18$ & $-84 \pm 22$ & $-50 \pm 22$ & $-34.8 \pm 0.6$ \\
& $\mathrm{PI}(3,5) \mathrm{P}_{2}$ & 4 & $0.30 \pm 0.03$ & $1.14 \pm 0.29$ & $-171 \pm 26$ & $-137 \pm 27$ & $-34.2 \pm 0.7$ \\
\hline ScHsv2 & $\mathrm{PI} \mathrm{P}$ & 5 & $0.58 \pm 0.09$ & $0.40 \pm 0.05$ & $-89 \pm 8$ & $-53 \pm 9$ & $-35.7 \pm 1.0$ \\
& $\mathrm{PI}(3,5) \mathrm{P}_{2}$ & 5 & $0.73 \pm 0.05$ & $0.03 \pm 0.01$ & $-62 \pm 4$ & $-18 \pm 5$ & $-44.1 \pm 1.0$ \\
\hline
\end{tabular}

The ITC titration curves for PaAtg18 binding to both PI3P and $\mathrm{PI}(3,5) \mathrm{P}_{2}$ are given in Figure 4.12, A and B. For PI3P, it can be observed that the heat released is weak under these conditions; weaker than the $0.1 \mu \mathrm{cal} / \mathrm{s}$ threshold limit for a reliable measurement. This must be taken into consideration when looking at thermodynamical parameters obtained from fitting this curve. The titration curves were fitted (Figure 4.12, C) and values for binding affinity and stoichiometry, entrophy and enthalphy were determined (Table 4.3). For a better comparison between the binding parameters for the two phosphoinositides investigated, histograms were drawn in Figure 4.12, D for the enthalphic term $(\Delta \mathrm{H})$, entropic term -T $\Delta \mathrm{S}$ and Gibbs free energy $(\Delta \mathrm{G})$. It can be observed that the enthalpic and entropic contributions are slightly larger for $\mathrm{PI}(3,5) \mathrm{P}_{2}$ binding compared to PI3P binding. Moreover, the entropy is unfavorable to binding, while the binding is enthalpically driven. Moreover, the $\mathrm{PaAtg} 18 \mathrm{PI}(3,5) \mathrm{P}_{2}$ binding is stronger than the PI3P binding since the latter has a binding affinity of $0.29 \mu \mathrm{M}$, while the former has a binding affinity three times higher, $0.11 \mu \mathrm{M}$. The binding stoichiometry could not be determined for PI3P binding, but for PI $(3,5) \mathrm{P}_{2} 2$ ligand molecules bind per PaAtg 18 protein.

The ITC titration curves K1Atg21 binding to both PI3P and PI(3,5) $\mathrm{P}_{2}$ are given in Figure 4.13, A and B. For both phosphoinositides, For both ligands, it can be observed that the heat released is weak under the measured conditions; the values are very close to the $0.1 \mu \mathrm{cal} / \mathrm{s}$ threshold limit for a reliable measurement. As before, the titration curves were fitted (Figure 4.13, C) and thermodynamic parameters determined (Table 4.3, Figure 4.13, D). Similar to PaAtg18, it can be observed that the enthalpic and entropic contributions are larger for $\mathrm{PI}(3,5) \mathrm{P}_{2}$ binding compared to PI3P binding. The entropy is unfavorable to binding, while the binding is enthalpically driven. Furthermore, the K1Atg21 binding to the two phosphoinositides investigated is approximatively equal 
PaAtg18

A

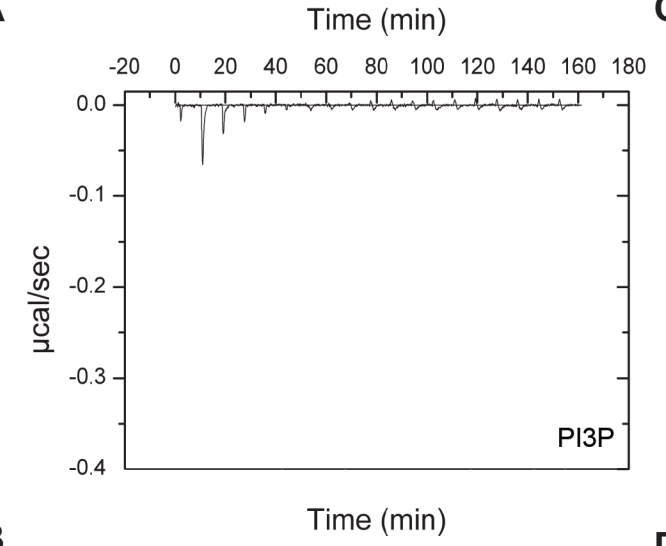

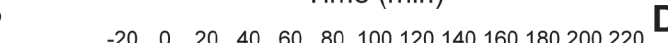

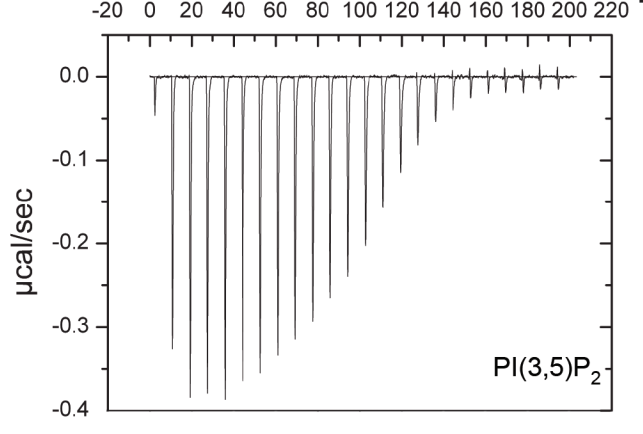

C
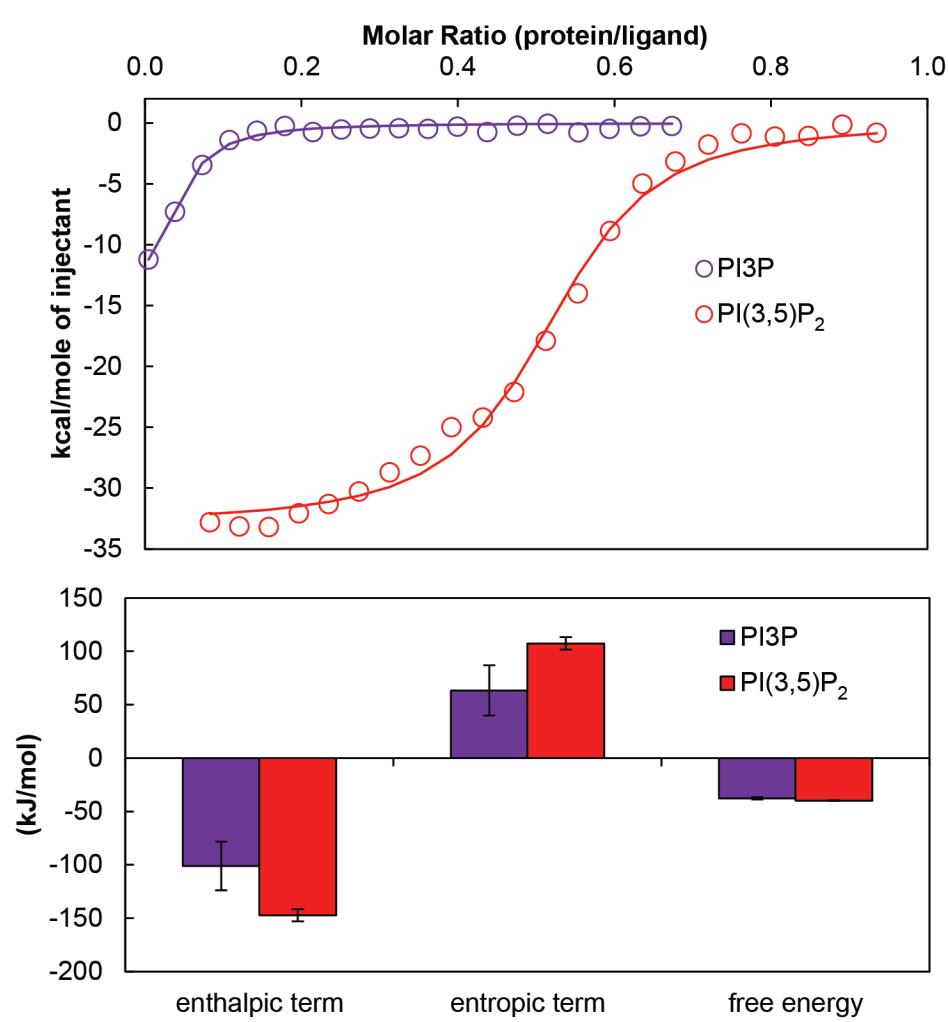

Figure 4.12: ITC measurements for PaAtg18 with SUVs containing PI3P or PI(3,5) $P_{2}$.

ITC curves are shown from titrations of PaAtg18 into SUVs containing 2\% PI3P (A) or PI(3,5) $\mathrm{P}_{2}$. Liposomes were composed of PC:PE:Texas-Red-PE:PI (72:24:2:2, weight ratio). Data was fitted with a One Set of Sites fitting model (C). The entalphic, entropic and energy terms are represented through histograms for both $\mathrm{PI} 3 \mathrm{P}$ and $\mathrm{PI}(3,5) \mathrm{P}_{2}$ (D). Error bars represent standard errors of the mean (SEM). 


\section{KIAtg21}

A

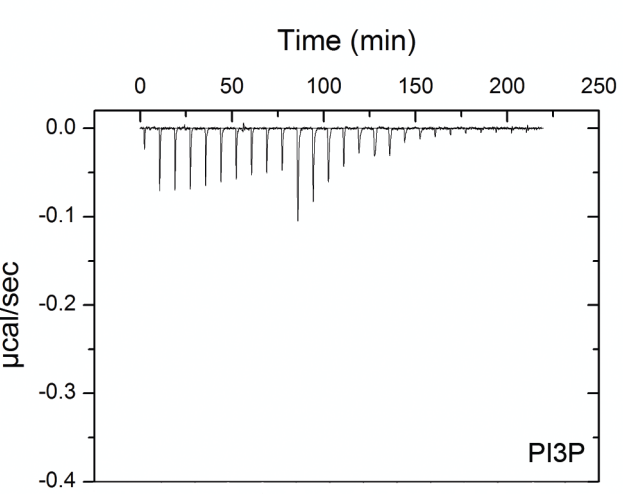

B

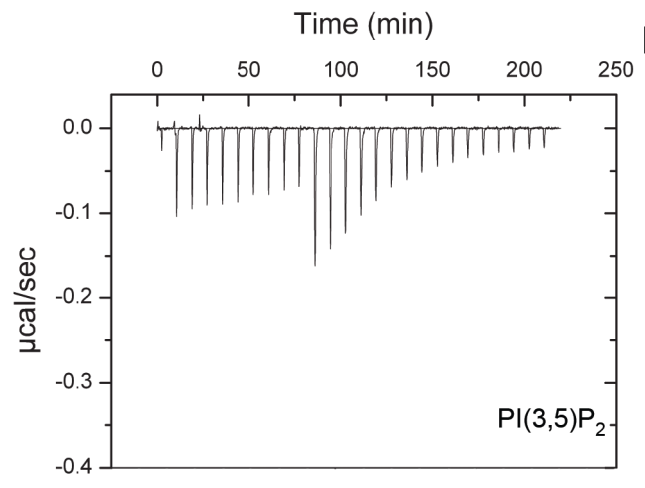

C

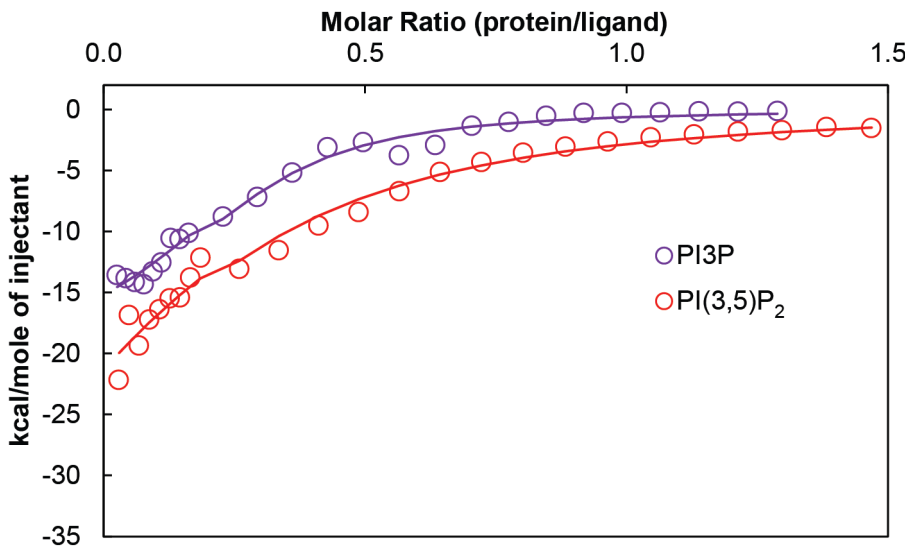

D

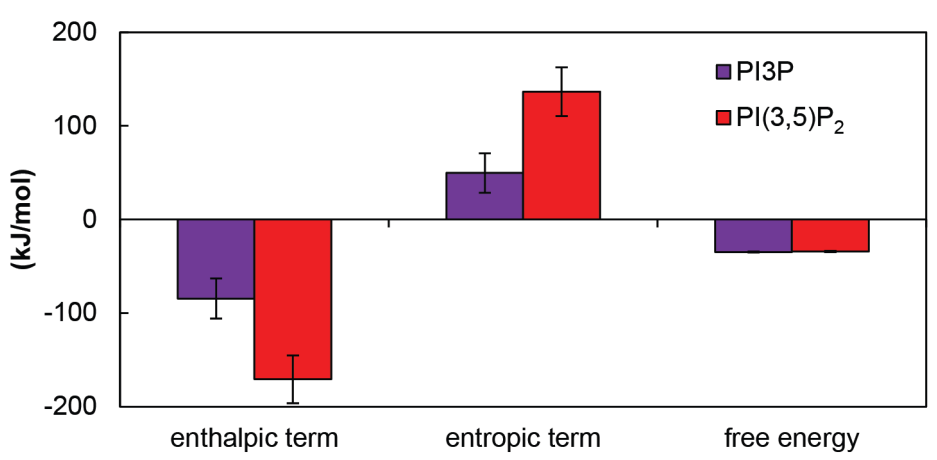

Figure 4.13: ITC measurements for KIAtg21 with liposomes containing PI3P or PI(3,5) $\mathbf{P}_{2}$.

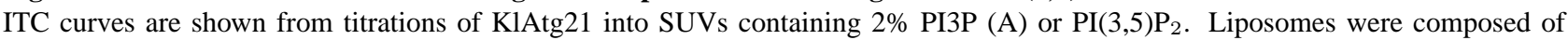
PC:PE:Texas-Red-PE:PI (72:24:2:2, weight ratio). Data was fitted with a One Set of Sites fitting model (C). The entalphic, entropic and energy terms are represented through histograms for both $\mathrm{PI} 3 \mathrm{P}$ and $\mathrm{PI}(3,5) \mathrm{P}_{2}(\mathrm{D})$. Error bars represent standard errors of the mean (SEM). 
in strength. The KlAtg21 binding affinity to $\mathrm{PI} 3 \mathrm{P}$ is $0.86 \mu \mathrm{M}$, while to $\mathrm{PI}(3,5) \mathrm{P}_{2}$ is $1.14 \mu \mathrm{M}$. According to the binding stoichiometries measured, 2.3 PI3P molecules bind to each $\mathrm{K} 1 \mathrm{Atg} 21$, while $3.3 \mathrm{PI}(3,5) \mathrm{P}_{2}$ ligand molecules bind per protein molecule.

\section{ScHsv2}

A

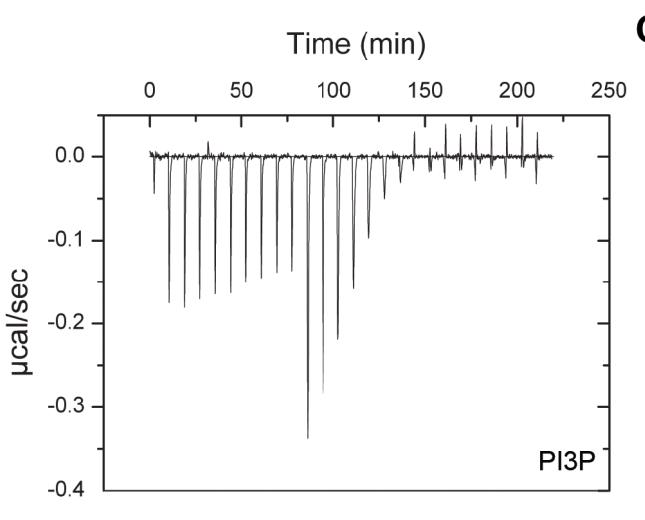

B

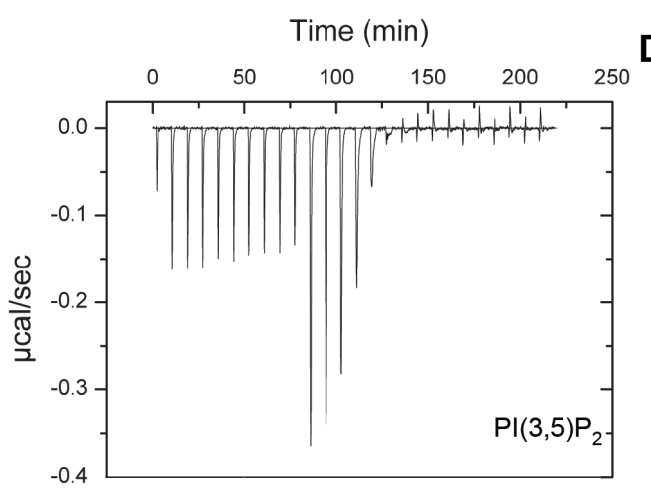

C
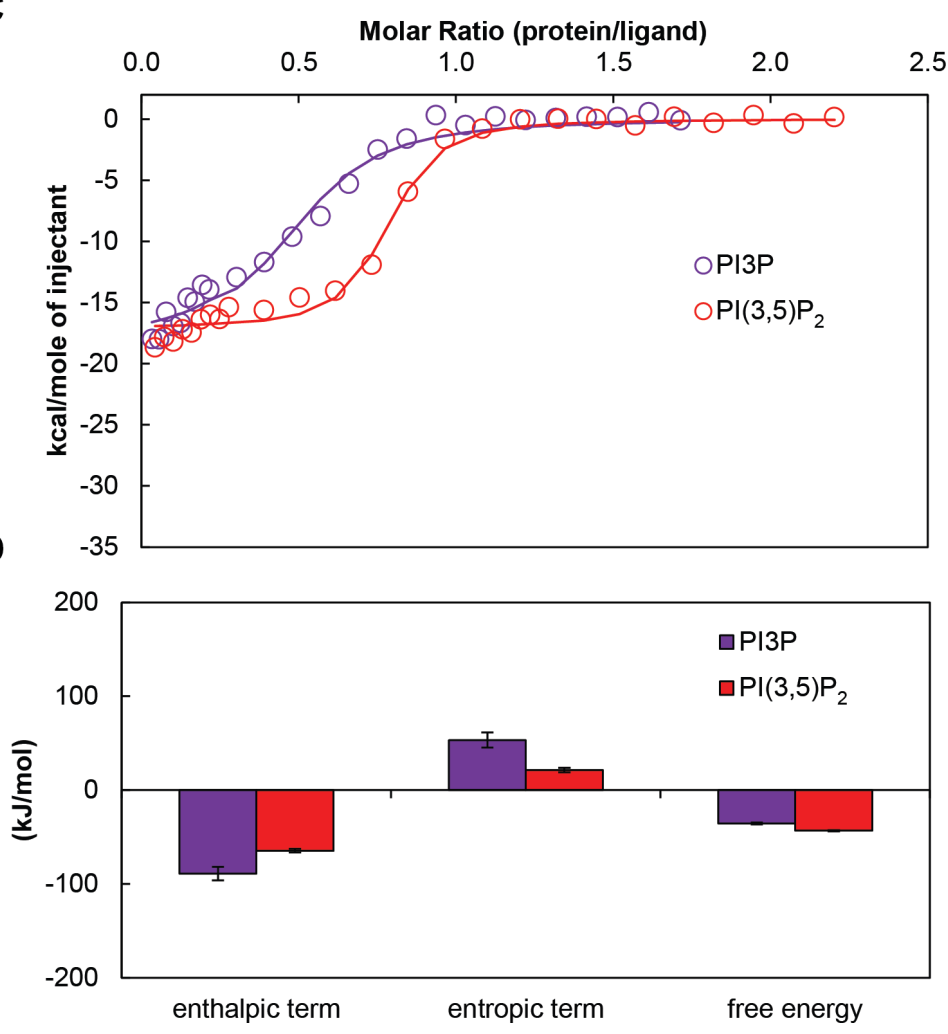

Figure 4.14: ITC measurements for ScHsv2 with SUVs containing PI3P or PI(3,5) $\mathbf{P}_{2}$.

ITC curves are shown from titrations of ScHSv2 into SUVs containing 2\% PI3P (A) or PI(3,5) $\mathrm{P}_{2}$. Liposomes were composed of PC:PE:Texas-Red-PE:PI (72:24:2:2, weight ratio). Data was fitted with a One Set of Sites fitting model (C). The entalphic, entropic and energy terms are represented through histograms for both $\mathrm{PI} 3 \mathrm{P}$ and $\mathrm{PI}(3,5) \mathrm{P}_{2}$ (D). Error bars represent standard errors of the mean (SEM).

Similar ITC measurements and analysis as for PaAtg18 and KlAtg21 was performed for ScHsv2 binding to $\mathrm{PI} 3 \mathrm{P}$ and $\mathrm{PI}(3,5) \mathrm{P}_{2}$ (Figure 4.14). Interestingly, in this case, it can be observed that the enthalpic and entropic contributions are smaller for $\mathrm{PI}(3,5) \mathrm{P}_{2}$ binding compared to $\mathrm{PI} 3 \mathrm{P}$ binding. However, the entropy is still unfavorable to binding, while the binding is enthalpically driven. ScHsv2 binding to $\mathrm{PI}(3.5) \mathrm{P}_{2}$ is more than ten times stronger $(0.03 \mu \mathrm{M})$ than to PI3P $(0.40 \mu \mathrm{M})$. According to the binding stoichiometries determined, 1.7 PI3P molecules bind to each ScHsv2, while 1.4 $\mathrm{PI}(3,5) \mathrm{P}_{2}$ ligand molecules bind per protein molecule.

\subsection{ITC measurements of wild type versus single binding site mutants of $S$. cerevisiae Hsv2 with PI3P containing liposomes}

Single binding site mutants of ScHsv2 were titrated in PI3P containing SUVs. The resulting ITC titration curves are shown in Figure 4.15, A and B. For ScHsv2(H294A), it can be observed that the heat released is weak under these conditions; weaker than the $0.1 \mu \mathrm{cal} / \mathrm{s}$ threshold limit for a reliable measurement. Low heat 
is also released in the case of ScHsv2(R264A) mutant, but the values are above the measurement threshold. This must be taken into consideration when discussing the resulting binding affinities determined from fitting these titration curves (Figure 4.15, C). The binding affinities determined are summarized in Table 4.4, Both mutants have much lower affinities for PI3P than wild type ScHsv2. ScHsv2(R264A) bound almost 30 times weaker than the wild type $\left(\mathrm{K}_{d}=10.9 \mu \mathrm{M}\right)$ while the ScHsv2H294A had a 15 -fold decreased binding $\left(\mathrm{K}_{d}=5.9\right.$ $\mu \mathrm{M})$ when compared to the wild type protein.

\section{ScHsv2}

A

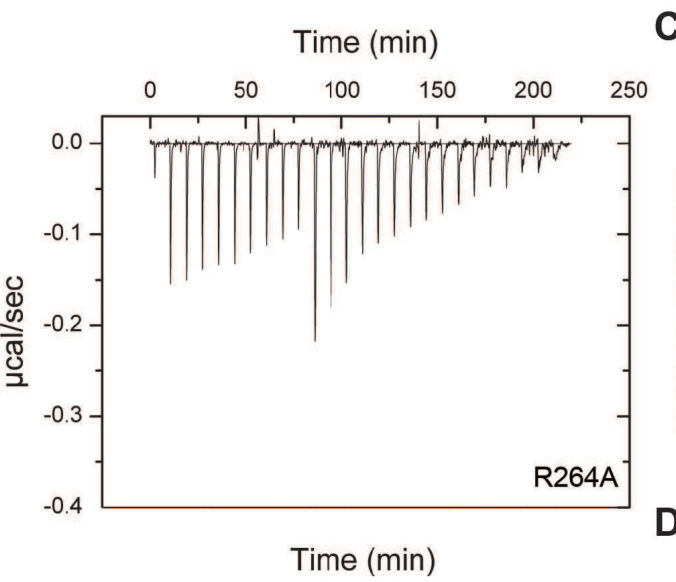

B

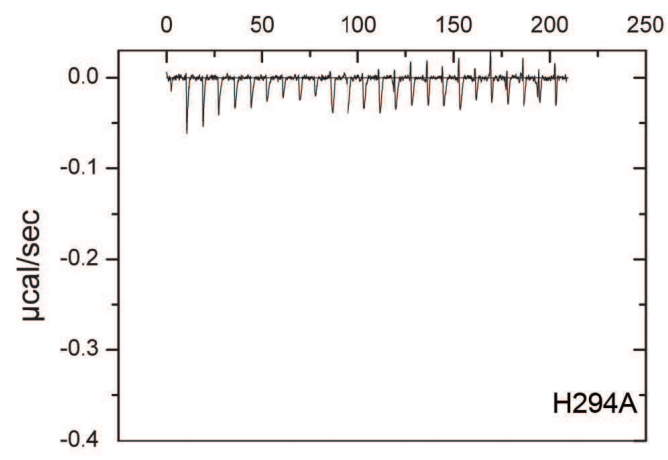

C
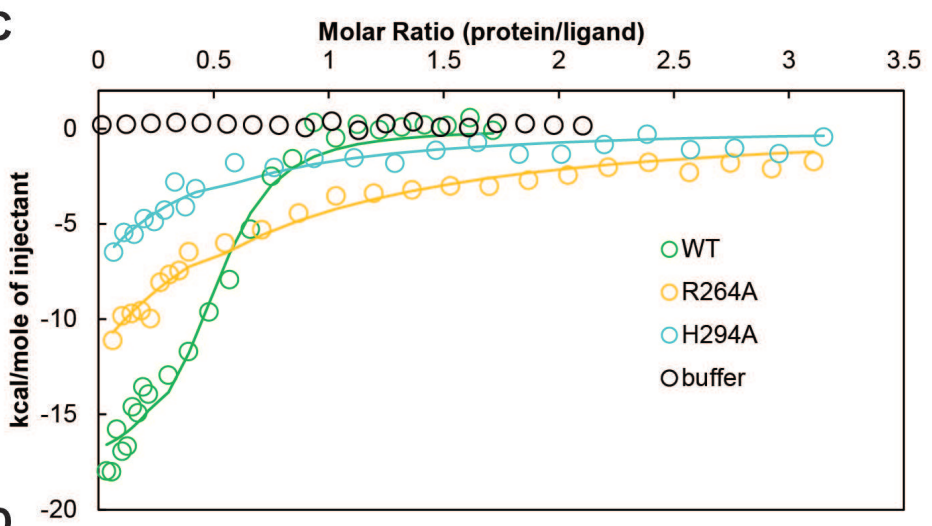

$-20$

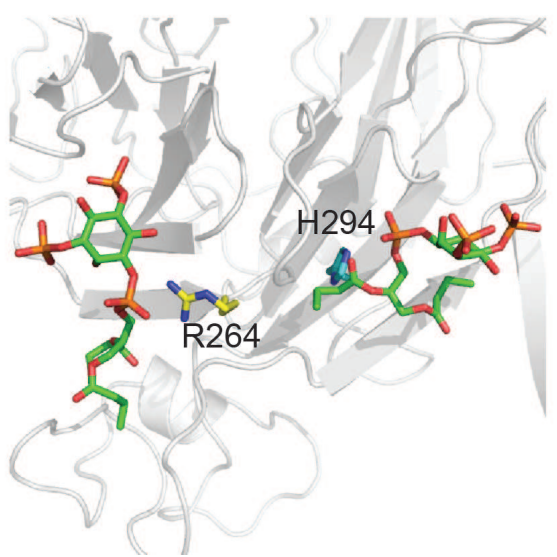

Figure 4.15: ITC measurements of single binding site ScHsv2 mutants with liposomes containing PI3P

ITC curves are shown from titrations of ScHsv2 mutants, ScHsv2(R264A) (A) and ScHsv2(H294A) into SUVs containing 2\% PI3P. Liposomes were composed of PC:PE:Texas-Red-PE:PI (72:24:2:2, weight ratio). Data was fitted with a One Set of Sites fitting model (C). Role of R264 and H294 in PI binding as shown in the homology model of ScHsv2 (D).

Table 4.4: ITC determined binding affinities for wild type ScHsv2 and its single sites mutants when titrated in PI3P containing SUVs. $\mathrm{n}$ - number of measurements.

\begin{tabular}{ccr}
\hline ScHsv2 & $\mathbf{n}$ & $\mathbf{K}_{d}(\mu \mathbf{M})$ \\
\hline wild type & 5 & $0.4 \pm 0.1$ \\
R264A & 4 & $10.9 \pm 1.2$ \\
H294A & 4 & $5.9 \pm 1.6$ \\
\hline
\end{tabular}




\section{Structural characterization of $P$. angusta $\operatorname{Atg} 18$}

\subsection{Crystallization of $P$. angusta Atg18}

\subsubsection{Full length $P$. angusta Atg18 crystallization}

Commercial crystallization screens in 96-wells were set with a pipetting robot. A single hit was obtained as summarized in Table 5.1. Crystals form in a protein concentration dependent manner: the more concentrated the protein, the ticker and longer the needles are (Figure 5.1). The crystals were then reproduced in a 24-well format, however they were too small for diffraction measurements. Grid screen were set up for optimization. The Na-acetate buffer $\mathrm{pH}$ was varied between 4.2 and 5.2, while the ammonium citrate dibasic precipitant concentration was varied between 0.4 and $1.9 \mathrm{M}$. In addition, as an alternative buffer, MES was tried with a varying $\mathrm{pH}$ between 5.2 and 7.1. Small needles were obtained in a range of conditions. These were used for streak seeding and microseeding. While small improvement could be observed, crystals did not grow sufficiently.

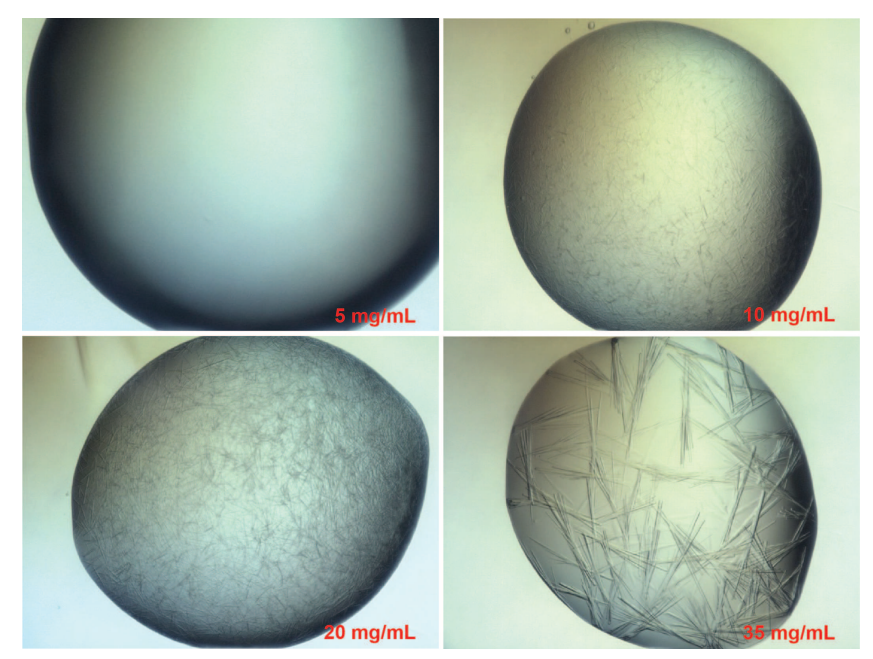

Figure 5.1: Initial crystallization hit for full length PaAtg18. Crystals were grown in sitting drops in a 96-wells plate format. The mother liquor composition is summarized in Table 5.1. 
Table 5.1: Sample crystallization conditions for full length PaAtg18

\begin{tabular}{rrr}
\hline Condition & Mother liquor composition \\
\hline & Ammonium citrate dibasic & $1 \mathrm{M}$ \\
Figure 5.1 & $0.1 \mathrm{M}$ Na-acetate trihydrate & $\mathrm{pH} \mathrm{4.6}$ \\
\hline \multirow{3}{*}{ Figure 5.2 } & Ammonium citrate dibasic & $1.5 \mathrm{M}$ \\
& $0.1 \mathrm{M}$ Na-acetate trihydrate & $\mathrm{pH} \mathrm{5.0}$ \\
& $\begin{array}{r}1,6-\text { diaminohexane } \\
\text { streak seeding }\end{array}$ \\
\hline
\end{tabular}

Next, additives were screened and improvements in the size of the crystal needles were observed. The best diffracting crystal was obtained in the presence of $15 \% \mathrm{w} / \mathrm{v}$ 1,6-diaminohexane and diffracted to $7.7 \AA$ (Figure 5.2).

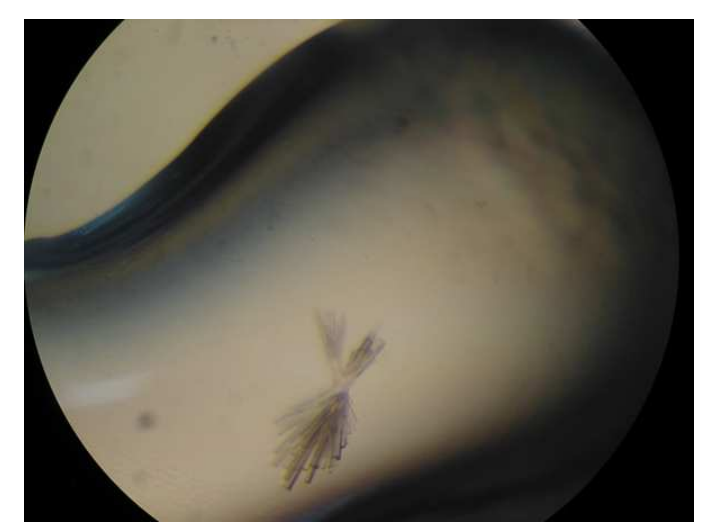

Figure 5.2: Full length PaAtg18 crystal diffracting to $7.7 \AA$. Crystal was grown in a hanging drop in a 24-wells plate format. The crystallization condition is summarized in Table 5.1 .

Since the ammonium citrate dibasic/Na-acetate condition could not be further improved, alternative strategies were tried, as schematically summarized in Figure 5.3 together with the number of successful crystal hits. The conditions for the crystal hits are summarized in Appendix table 8.8

An N-terminal peptide from P. angusta Atg2 was tested for co-crystallization. However, precipitate formed. I have tested the same peptide for correct folding in the expected $\alpha$-helix by measuring a CD spectrum. However, the peptide presented the spectrum of a random coil. Random microseeding [198] was tried using the crystal needles previously obtained. However, the resulting crystals were also short and thin needles that could not be further optimized.

\subsubsection{In situ proteolysis for crystallization of P. angusta Atg18}

As a next crystallization strategy, in situ proteolysis [199] was tested. Dr. Ricarda Busse tested the PaAtg18 cleavage pattern as a result of protease treatment [192]. Based on these experiments, trypsin, subtilisin and Proteinase $\mathrm{K}$ were chosen for in situ proteolysis. Crystals were obtained with subtilisin and Proteinase K. Initial hits are shown in Figure 5.4 and crystallization conditions are summarized in Table 5.2. Successful conditions are summarized in Appendix table 8.8. Interestingly, all conditions contained phosphate. The buffer 


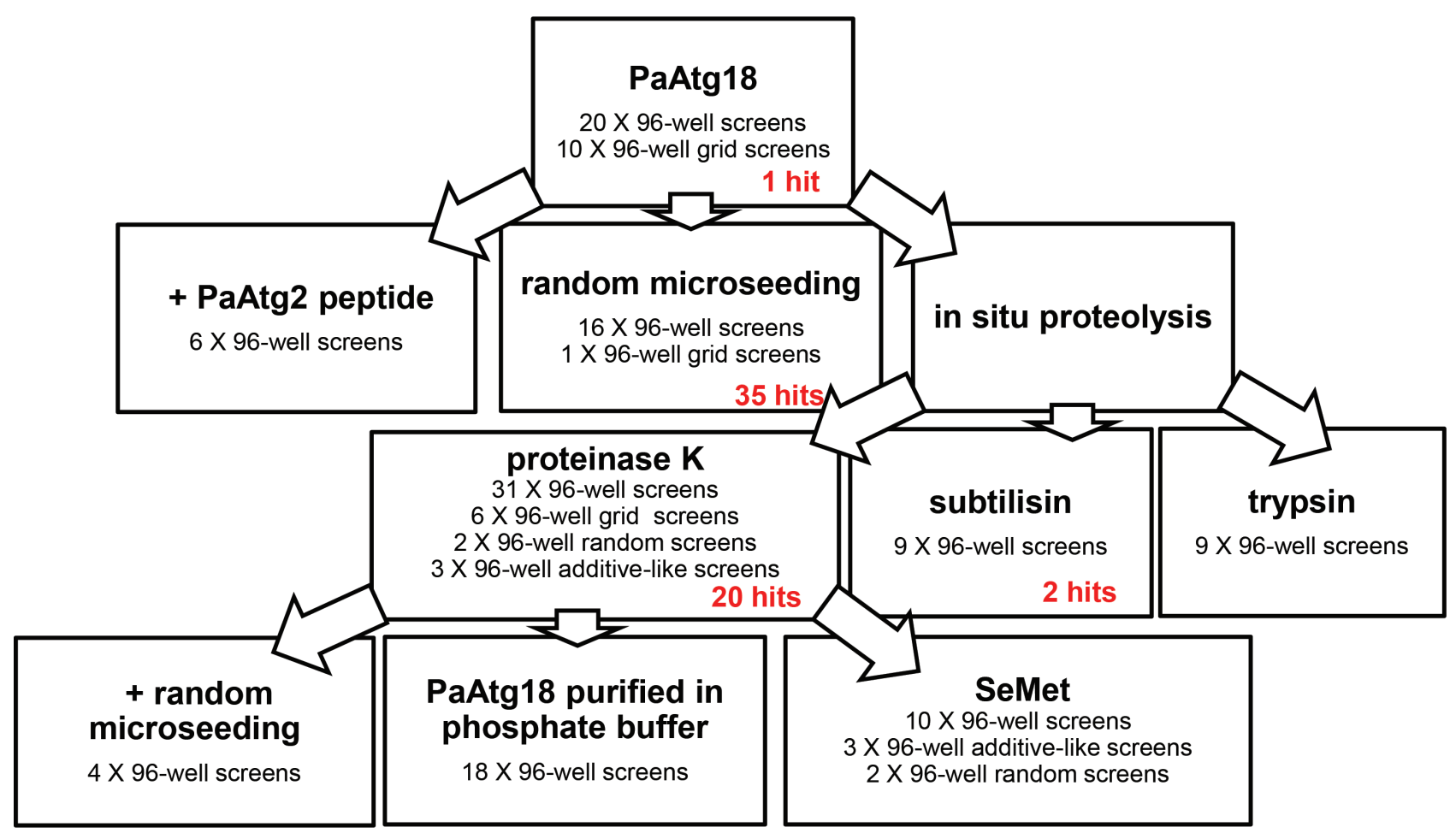

Figure 5.3: Crystallization strategies tried for PaAtg18 .

$\mathrm{pH}$ did not play a major role. All crystals grew as clusters of multiple plates originated from spherulites that appeared a few days before the first crystals. These clusters were they diffracted between $2 \AA$ and $3 \AA$.

The most promising condition was $20 \%$ PEG 8000, $0.2 \mathrm{M} \mathrm{NaCl}, 0.1 \mathrm{M}$ phosphate-citrate buffer, $\mathrm{pH}$ 4.2. Crystals only grew in the commercially available crystallization mixture. The commercial mixture made it possible to reproduce the crystals in 24-well format. However, the commercial solution mixture (Emerald) allowed no optimization by varying the buffer $\mathrm{pH}$ or precipitant concentration. This is why additives and silver bullets were tested. Selenomethionine labeled PaAtg18 was purified and crystallized in the same conditions. A selection of selenomethionine labeled PaAtg18 crystals is shown in Figure 5.5

\subsection{Structure determination of P. angusta Atg18}

\subsubsection{Data collection and processing}

Crystals were fished, cryo-protected and flash cooled. Several cryoprotectants were tested for native PaAtg 18 crystals: $20 \%$ and $30 \%$ glycerol, $20 \%$ and $30 \%$ ethylene glycol, PEG400, PEG8000, PFPE (perfluoropolyether oil) and $10 \%$ xylitol $/ 10 \%$ sucrose $(\mathrm{w} / \mathrm{w})$. However, there were no significant differences in the quality of the diffraction data. Since single crystals were difficult to fish because they grew as clusters, the less viscous cryoprotectant was chosen, $30 \%$ ethylene glycol. Single crystals were removed from clusters with an acupuncture needle.

X-ray diffraction data were collected at $100 \mathrm{~K}$ at beamline X10SA (Swiss Light Source, Paul Scherrer Institute, Villigen, Switzerland). Data for structure determination were collected from both selenomethionine and native PaAtg18 crystals. After mounting of a single crystal for data collection, four test shots were taken 


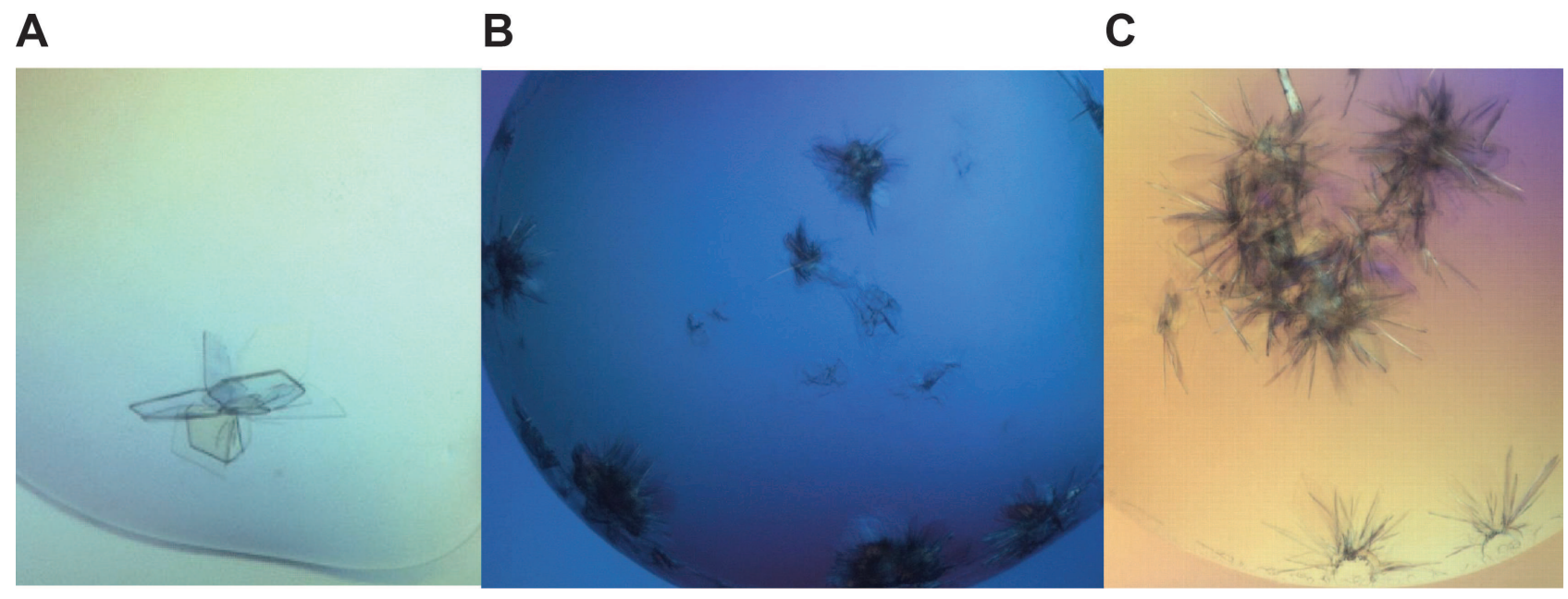

Figure 5.4: Selection of crystallization hits for PaAtg18 in situ proteolysis.

Crystals were grown in 96-wells plate in sitting drops. Crystallization conditions are summarized in Table 5.2

Table 5.2: Selection of successful conditions for in situ proteolysis of PaAtg18. The resulting crystals are shown in Figure 5.4

\begin{tabular}{rrr}
\hline Condition & Mother liquor composition \\
\hline & PEG 8000 & $20 \%(\mathrm{w} / \mathrm{v})$ \\
Figure 5.4, A & $\mathrm{NaCl}$ & $0.2 \mathrm{M}$ \\
& 0.1 M citrate phosphate buffer & $\mathrm{pH} 4.2$ \\
& di-sodium hydrogen phosphate & $0.1 \mathrm{M}$ \\
& proteinase K & $1: 2000$ \\
\hline & $0.1 \mathrm{M} \mathrm{Na}$-acetate trihydrate & $\mathrm{pH} 4.6$ \\
Figure 5.4, B & ammonium phosphate monobasic & $1.8 \mathrm{M}$ \\
& proteinase K & $1: 2000$ \\
\hline & Na phosphate monobasic monohydrate & $0.8 \mathrm{M}$ \\
Figure 5.4, C & potassium phosphate monobasic & $0.8 \mathrm{M}$ \\
& $0.1 \mathrm{M}$ HEPES sodium & $\mathrm{pH} \mathrm{7.5}$ \\
& subtilisin & $1: 2000$ \\
\hline
\end{tabular}




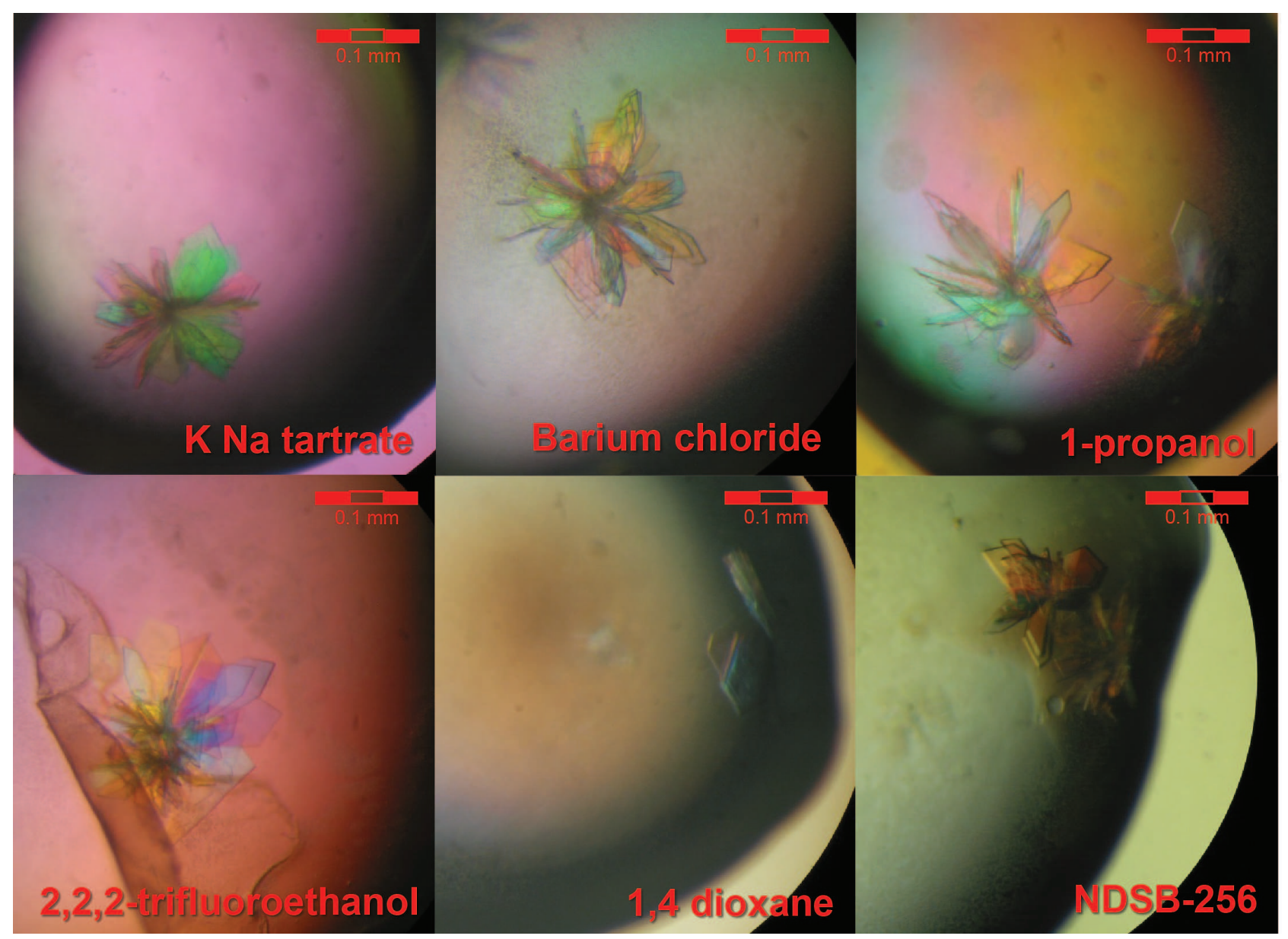

Figure 5.5: Selection of crystallization hits for selenomethionine labeled PaAtg18 in situ proteolysis.

Crystals were grown in Linbro 24-wells plates in hanging drops. The crystallization condition was 20\% PEG 8000, $0.2 \mathrm{M} \mathrm{NaCl}, 0.1 \mathrm{M}$ phosphate-citrate buffer, $\mathrm{pH}$ 4.2. The additive used for each condition is given in the respective panel. 
for indexing and determining an optimum collection strategy. The local go.com software written by Dr. M. Wang (SLS) was used for data processing at the beamline.

Since the crystals were thin, they diffracted anisotropically. Initially, data was collected with with an oscillation angle of $0.2^{\circ}$ over a $360^{\circ}$ rotation. However, during processing, these data sets did not have optimum statistics as the $\mathrm{I} / \sigma$ was below 10 and the completeness below $95 \%$. Next, the collection protocol was optimized such that the oscillation angle was $0.5^{\circ}$ and the crystal was rotated over a total range of $500^{\circ}$. This allowed collection of data sets with improved statistics for both native and selenomethionine labelled PaAtg 18 crystals.

For selenomethionine a fluorescence spectrum scan around the Se K absorption edge was taken to determine the optimum energy for data collection (Figure 5.6). For this, a protein crystal of the same purification batch as the ones used for collecting data sets was used. The Inflection, Peak, and remote 1 and remote 2 energies were determined with AUTOCHOOCH [200]. For the inflection, the determined value was $12662.4 \mathrm{eV}\left(\mathrm{f}^{\prime}=-10.690\right.$; $\left.\mathrm{f}^{\prime \prime}=3.190\right)$, while for the peak it was $12665.6 \mathrm{eV}(\mathrm{f}=-8.220$; f"=6.780).

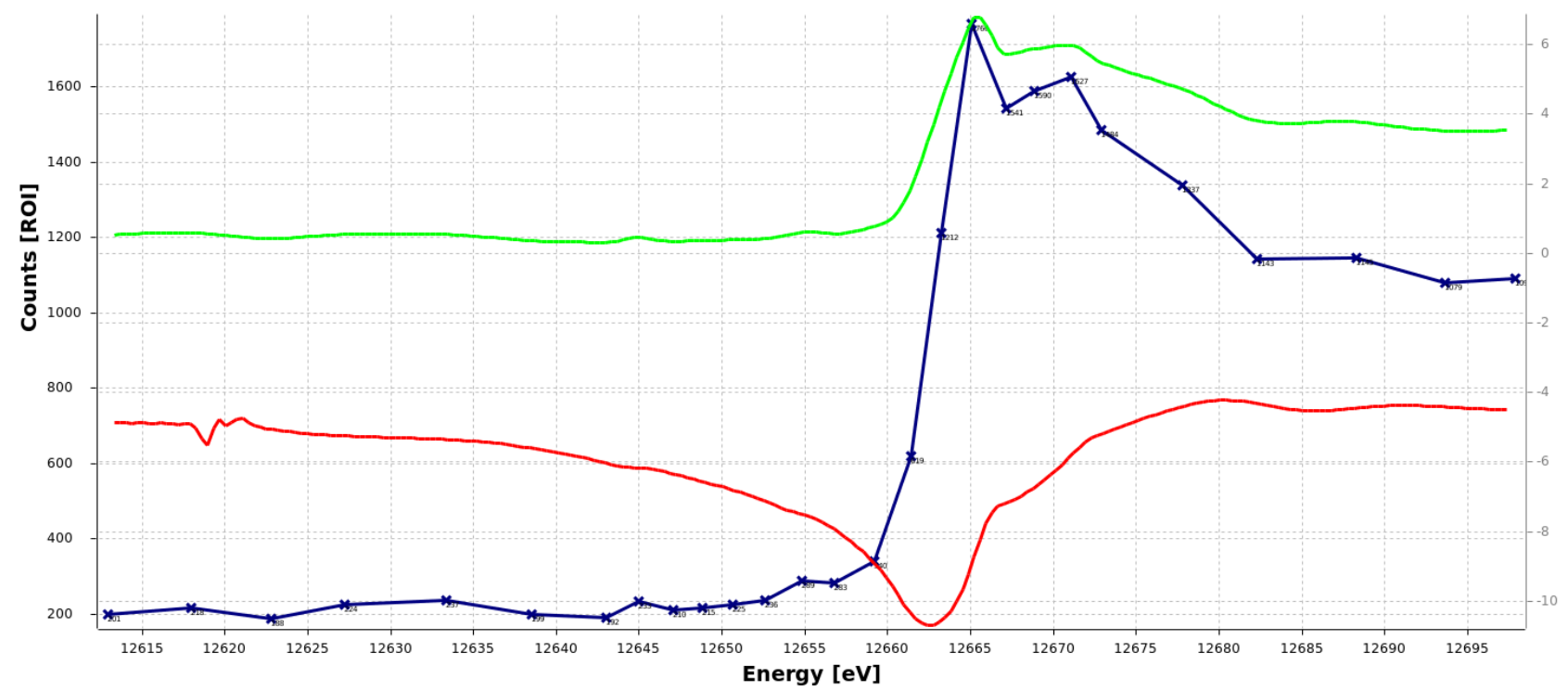

Figure 5.6: Fluorescence spectrum and a scan of Selenomethionine labeled PaAtg18 crystal. Counts (ROI) are represented in blue. Fitted f' (red) and fitted f"' (green) are shown.

A SAD dataset of $500^{\circ}$ was collected at the Se peak wavelength. The data collection parameters are summarized in Table 5.3). A diffraction pattern is given in Figure 5.7. Data were processed with XDS [157]. In order to index the weak diffraction spots, the pixel size was set to 3 .

Table 5.3: Data collection parameters for the 1.8 Å selenomethionine PaAtg 18 crystal

\begin{tabular}{cc}
\hline Detector distance $(\mathrm{mm})$ & 325 \\
$\Delta \phi\left(^{\circ}\right)$ & 0.5 \\
Exposure time $(\mathrm{s})$ & 0.15 \\
Beam intensity/attenuators & 0.0993 \\
$\lambda(\AA)$ & 0.98 \\
No of frames & 1000 \\
\hline
\end{tabular}




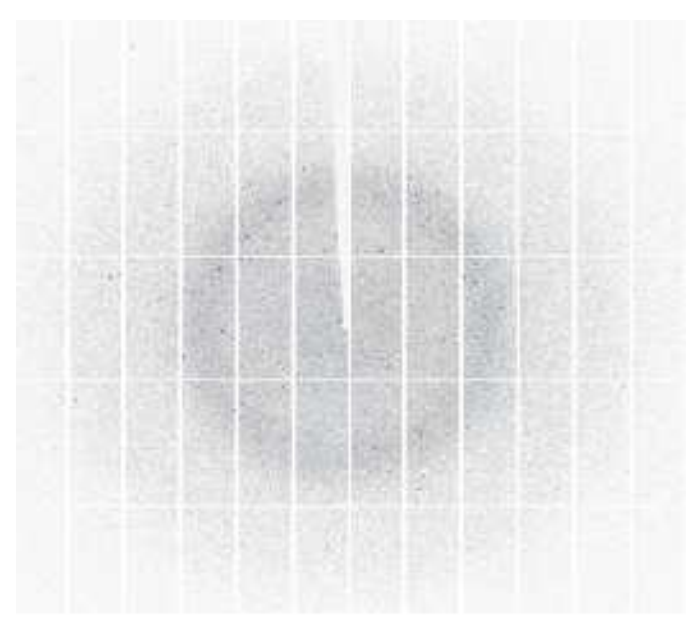

Figure 5.7: Diffraction image of PaAtg18 crystals.

The space group was determined as P1. Dimensions of the unit cell, data and refinement statistics are summarized in Table 5.4 .

\subsubsection{SAD phasing with selenomethionine labeled $P$. angusta Atg18 crystals}

Scaled data was converted to unmerged CCP4F format using XDSCONV. With this file, I tried to phase the data using SHELX programs [201]. However, in order to offer a proper result, SHELX needs a good approximation for the molecular weight of the crystallized protein. Since in situ proteolysis was used, it was unknown which regions were cleaved by the proteases, hence an estimation of the molecular weight was difficult to get an estimation of the water content of the crystals. In order to have an indication of the molecular weigth of the molecule in the unit cell, I harvested crystals and probed them on an SDS-PAGE gel. A smeary pattern was obtained, due to the high PEG 8000 content and no protein band was distinguishable.

Next, AutoSol package from Phenix was used [158]. The same data set from the selenomethionine labeled crystal was processed as native. I used the built structure as a search model to determine the high resolution structure at $1.8 \AA$. A sample of the electron density map is shown in Figure 5.8, The cutoff of the anomalous signal was determined at 3.5 while the high resolution limit was determined to be $2.7 \AA$

Phenix found eleven Se sites, eight of them with occupancies above 0.8 (Table 5.5). The obtained experimental electron density map phasing model had an overall model-map correlation of 0.847 . The initial model contained two molecules per asymmetric unit.

\subsubsection{Refinement and structure validation}

Phenix was used for refinement [158] and Coot [159] was used for model building. Several cycles of refinement with Phenix resulted in a final structure refined to R-work/R-free of 20/23\%. Water molecules were added manually and using the tools in Coot. Disordered side chains were modelled as alanines. For each of the two molecules present in an asymetric unit, a phosphate and a potassium ion were added. Validation of the structure was done using the Molprobity plugin in Phenix and the tools available in Coot. The refinement statistics were compared to structures in the PDB with a similar resolution cutoff (Figure 5.9, A). All parameters lie in the range. However, the average B factor is high for a $1.8 \AA$ resolution structure. Geometry of the refined 
Table 5.4: Diffraction data and refinement statistics of the 1.8 A selenomethionine PaAtg18 crystal

\begin{tabular}{|c|c|}
\hline Space group & $\mathrm{P} 1$ \\
\hline \multirow[t]{2}{*}{ Cell dimensions $(\AA)$} & $a=57.8, b=57.9, c=61.8$ \\
\hline & $\alpha=84.5^{\circ}, \beta=81.6^{\circ}, \gamma=87.1^{\circ}$ \\
\hline Resolution range $(\AA)$ & $45.0-1.8(1.87-1.8)$ \\
\hline Total reflections & $347538(53750)$ \\
\hline Unique reflections & $71072(6925)$ \\
\hline Multiplicity & 4.9 \\
\hline Completeness (\%) & $97.4(94.3)$ \\
\hline Mean $\mathrm{I} / \sigma$ & $15.5(2.9)$ \\
\hline Wilson B-factor $\left(\AA^{2}\right)$ & 24 \\
\hline $\mathrm{R}-\operatorname{sym}(\%)$ & $4.8(38.4)$ \\
\hline \multicolumn{2}{|l|}{ Refinement } \\
\hline R-work & $0.20(0.27)$ \\
\hline $\mathrm{R}$-free & $0.23(0.31)$ \\
\hline molecules per a.u. & 2 \\
\hline Protein segments present & $33-179 ; 201-297 ; 441-467 ; 485-524$ \\
\hline \multicolumn{2}{|l|}{ No of atoms present } \\
\hline macromolecules & 4678 \\
\hline ligands & 38 \\
\hline water & 417 \\
\hline \multicolumn{2}{|l|}{ RMS } \\
\hline bonds & 0.009 \\
\hline angles & 1.14 \\
\hline \multicolumn{2}{|l|}{ B-factors $\left(\AA^{2}\right)$} \\
\hline overall & 30.9 \\
\hline macromolecules & 30.2 \\
\hline ligands & 47.1 \\
\hline solvent & 37.2 \\
\hline \multicolumn{2}{|l|}{ Structure validation } \\
\hline Ramachandran favored (\%) & 98 \\
\hline Ramachandran outliers (\%) & 0 \\
\hline Clashscore & 2.65 \\
\hline
\end{tabular}

Table 5.5: Fractional coordinates and occupancies for Se atoms

\begin{tabular}{lrrrr}
\hline SE & -0.389 & -0.388 & -0.095 & 1.73 \\
SE & -0.544 & -0.144 & -0.047 & 1.54 \\
SE & -0.424 & -0.768 & -0.962 & 1.68 \\
SE & 0.002 & -0.005 & 0.011 & 1.28 \\
SE & -0.248 & -0.847 & -0.037 & 1.29 \\
SE & -0.629 & -0.96 & -0.123 & 1.17 \\
SE & -0.792 & -0.343 & -0.036 & 0.96 \\
SE & -0.049 & -0.596 & -0.051 & 0.82 \\
SE & -0.046 & -0.622 & -0.025 & 0.72 \\
SE & -0.766 & -0.342 & -0.062 & 0.5 \\
SE & -0.008 & -0.584 & -0.08 & 0.27 \\
\hline
\end{tabular}




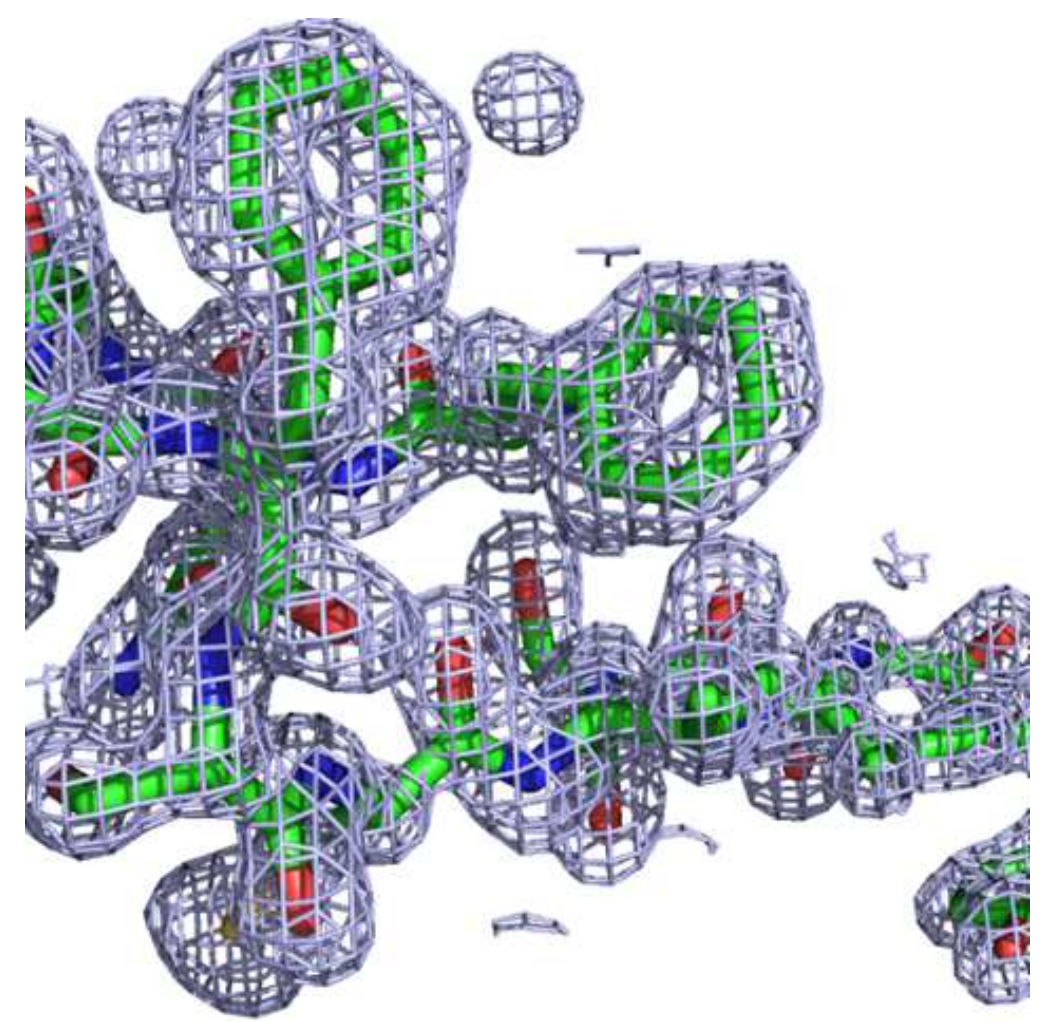

Figure 5.8: Experimental $2 \mathrm{mF}_{o}-\mathrm{DF}_{c}$ electron density map contoured at $1.0 \sigma$ with overlaid PaAtg18 structure at $1.8 \AA$

structure was analyzed in the Ramachandran plot (Figure 5.9. B). $98 \%$ of the residues are in the Ramachandran favored region with no outliers.

\subsubsection{P. angusta Atg18 structure analysis}

PaAtg 18 is a $\beta$-propeller with seven blades (Figure 5.10, A). It has a non-velcro closure, namely, the N- and C-termini are parts of distinctive blades, unlike most of the other known $\beta$-propellers. Each of the seven blades is formed by four anti-parallel $\beta$-strands. Three loops are absent in the crystal structure: 180-200, 298-440 and 468-484. The first loop is loop 4AB. The longest loop with almost 150 amino acids missing, is loop 6CD. Loop $7 \mathrm{AB}$ is also missing, which is more exposed than the rest of the loops as strands $7 \mathrm{~A}$ and $7 \mathrm{~B}$ are longer than the rest and protrude towards the outside of the $\beta$-propeller. Moreover, the first $32 \mathrm{~N}$-terminal amino acids are disordered.

There are two PaAtg18 molecules per asymmetric unit. They are very similar and overlay with an RMSD of $0.24 \AA$

The two arginines in the essential FRRG motif are pointing in opposite sides (Figure 5.12, A). In the structure, a phosphate was bound to PI binding site 2, while a larger density was found in binding pocket 1. Out of the compounds present in the crystallization condition and cryo-protectant, the potential crystal bound ligands were citrate or ethylene glycol. To distinguish,PaAtg18 crystals were fished and soaked in cryoprotectant solution that contain high amount of phosphate. Cryo-protection mixtures tested are summarized in Table 5.6 
A

\section{B}
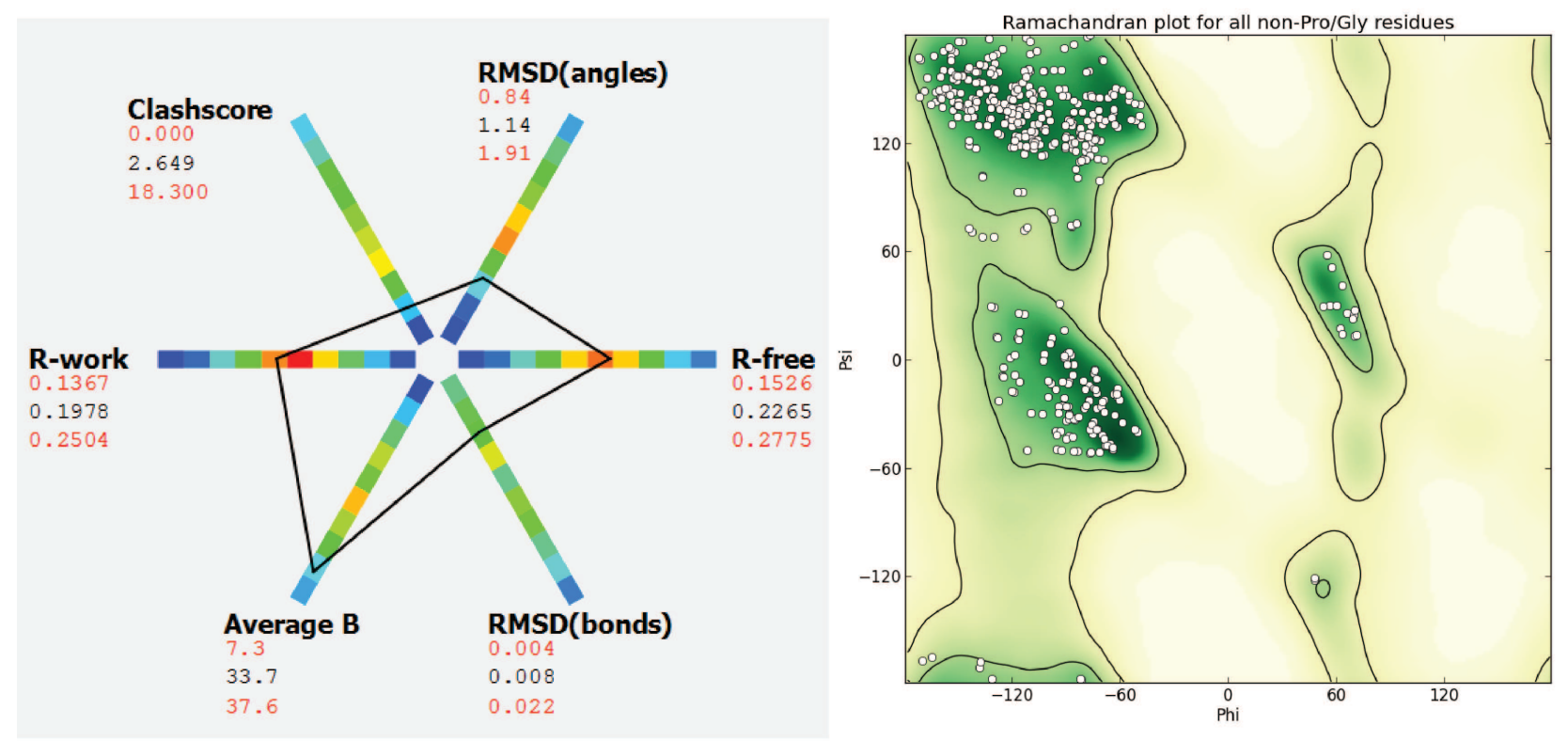

Figure 5.9: Refinement statistics for PaAtg18 structure. (A) Polygon plot by Phenix Selected statistics across PDB entries of similar resolution are compared with the determined structure. The expected range for the values are indicated in red numbers. The distribution of the structures over this range is shown in a heat map. (B) Ramachandran plot of all non Pro/Gly residues of PaAtg18.

Table 5.6: Summary of cryoprotectants tested in order to identify the compound in binding site 1

\begin{tabular}{rrrrrr}
\hline & cryo 1 & cryo 2 & cryo 3 & cryo 4 & cryo 5 \\
\hline PEG 8000 & $35 \%$ & $20 \%$ & $20 \%$ & $20 \%$ & $20 \%$ \\
NaCl & $200 \mathrm{mM}$ & $200 \mathrm{mM}$ & $200 \mathrm{mM}$ & $200 \mathrm{mM}$ & $200 \mathrm{mM}$ \\
phosphate & $260 \mathrm{mM}$ & $400 \mathrm{mM}$ & $100 \mathrm{mM}$ & - & $260 \mathrm{mM}$ \\
$\mathbf{p H}$ & 5 & 5 & 5 & 4.2 & 5 \\
citrate/phosphate buffer & - & - & - & $100 \mathrm{mM}$ & - \\
xylitol/sucrose & - & $10 \% / 10 \%(\mathrm{w} / \mathrm{w})$ & $10 \% / 10 \%(\mathrm{w} / \mathrm{w})$ & $10 \% / 10 \%(\mathrm{w} / \mathrm{w})$ & - \\
PEG 400 & - & - & - & - & $30 \%$ \\
\hline
\end{tabular}


A

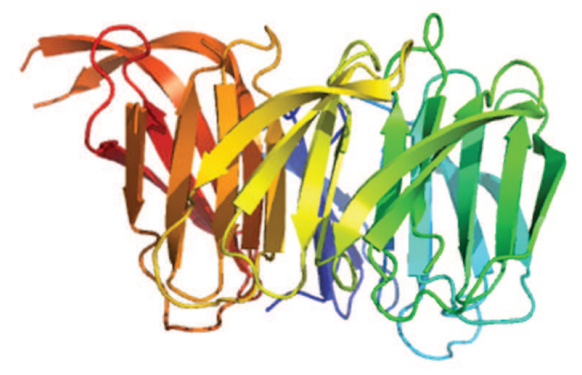

${ }_{90}^{\circ} €$

B

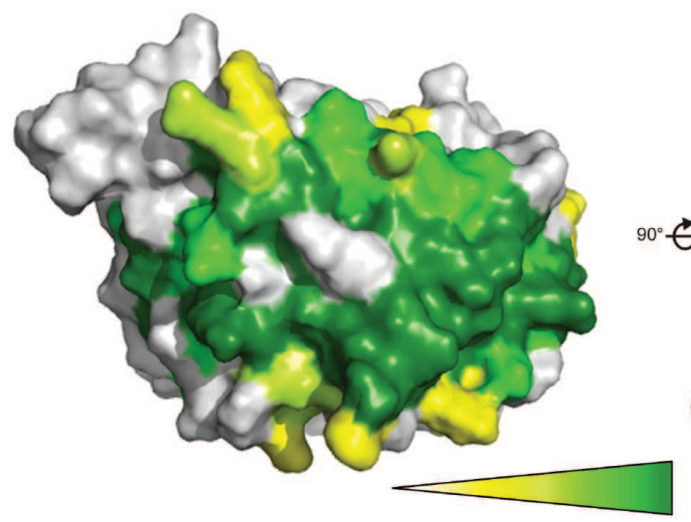

${ }_{90}^{\circ} €$

C
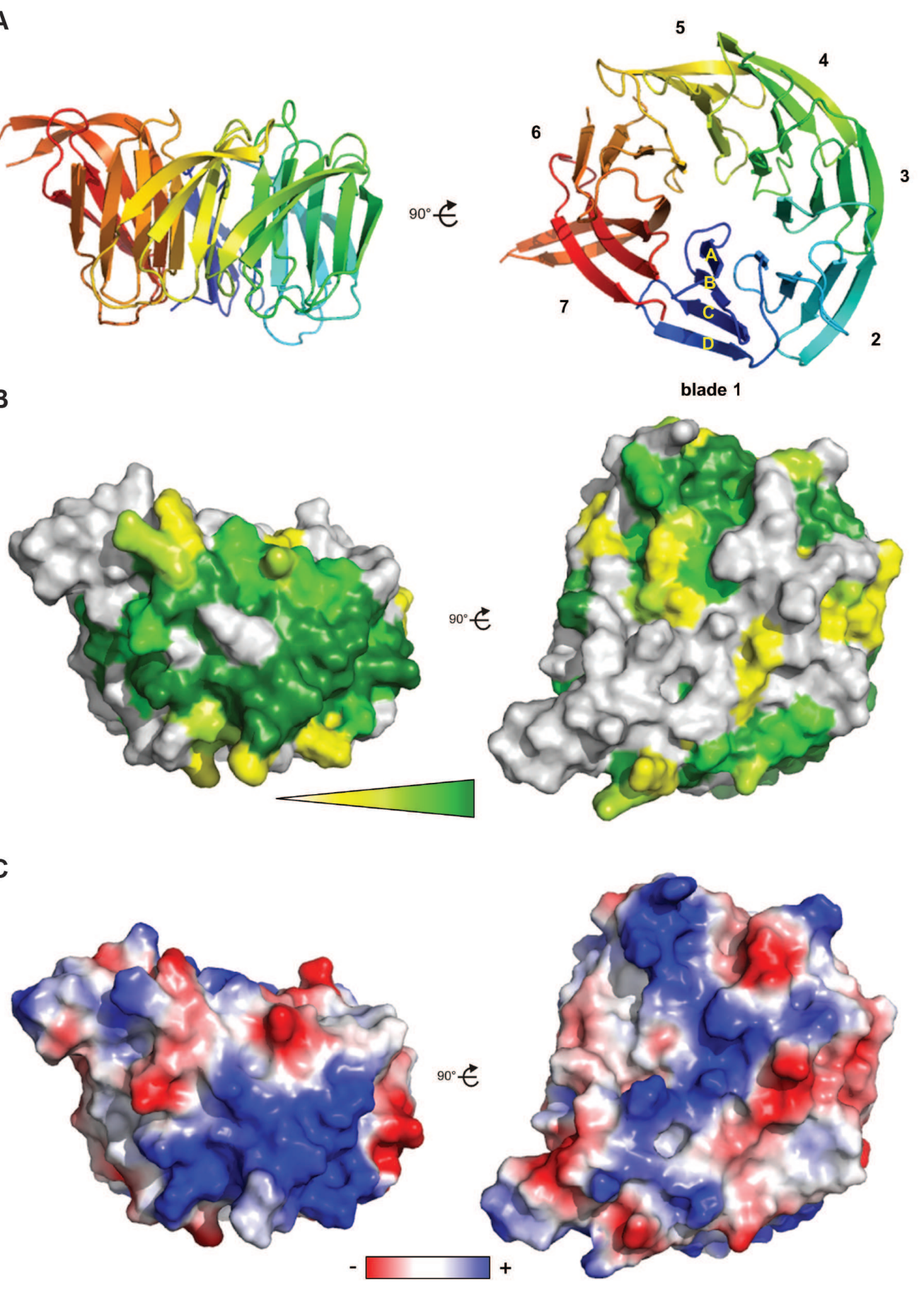

blade 1

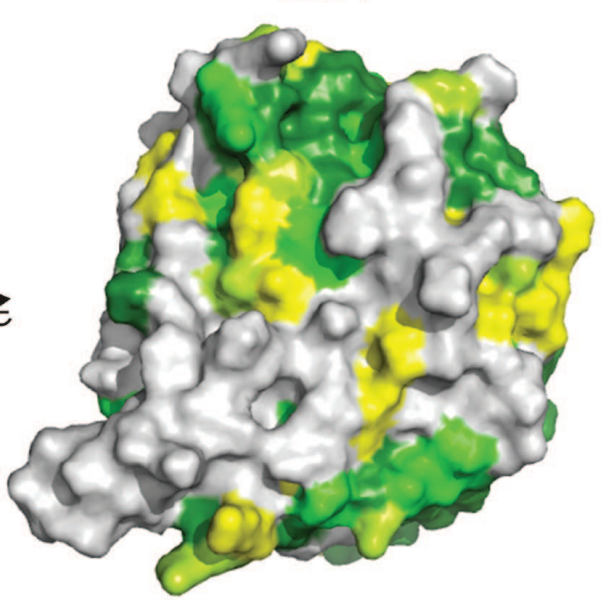

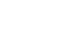

Figure 5.10: PaAtg18 structure analysis. (A) Overall structure, (B) surface conservation and (C) electrostatic surface potential of PaAtg18. The conservation of PaAtg18 is based on a sequence alignment of the Atg18 homologues from P. angusta, Pichia pastoris, S. cerevisiae, Thielavia terrestris, Trichoderma virens, Meyerozyma guilliermondii, Candida albicans, Dekkera bruxellensis, Wickerhamomyces ciferrii, Scheffersomyces stipites, Debaryomyces hansenii, Candida maltosa, Spathaspora passalidarum and Clavispora lusitaniae. For the electrostatic potential, side chains, which are disordered in the crystal structure were included. Figures are prepared with PyMol [184]. 
One data set was collected at resolution $2.3 \AA$ using cryoprotectant labeled as cryo 2 in Table 5.6 . The data collection conditions are summarized in Table 5.7 For this crystal, 10\% xylitol/10\% sucrose (w/w), 20\% PEG $8000,200 \mathrm{mM} \mathrm{NaCl}, 400 \mathrm{mM}$ phosphate $\mathrm{pH} 5$ was used as cryoprotectant solution. The data and refinement statistics are summarized in Table 5.8. In this structure, phosphates bound to both PI binding sites. This finding allowed me to exclude ethylene glycol as a binding partner, and I refined citrate as ligand bound at high resolution structure (Figure 5.11).

Table 5.7: Data collection parameters for the $2.3 \AA$ A selenomethionine PaAtg 18 crystal

\begin{tabular}{cc}
\hline Detector distance $(\mathrm{mm})$ & 325 \\
$\Delta \phi\left(^{\circ}\right)$ & 0.5 \\
Exposure time $(\mathrm{s})$ & 0.15 \\
Beam intensity/attenuators & 0.094 \\
$\lambda(\AA)$ & 0.98 \\
No of frames & 1500 \\
\hline
\end{tabular}

A

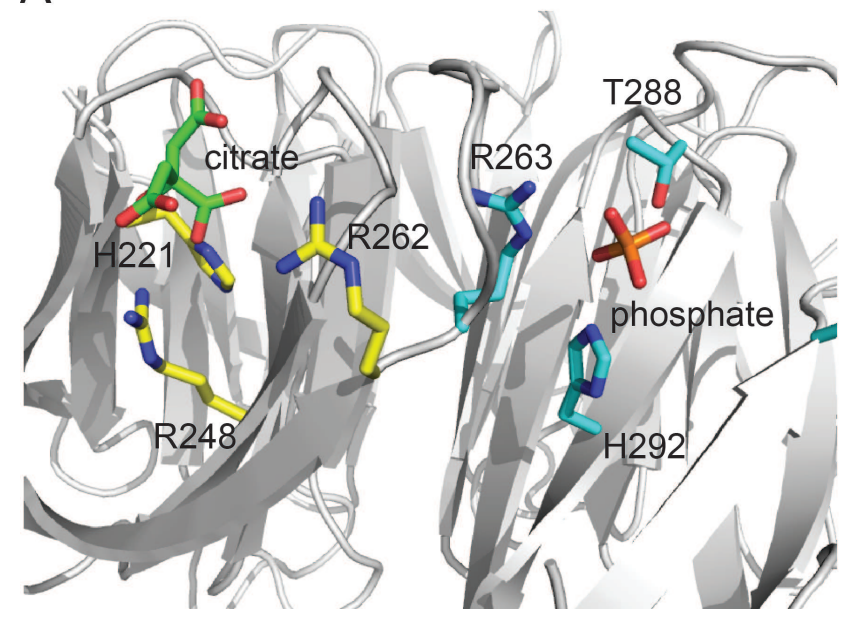

B

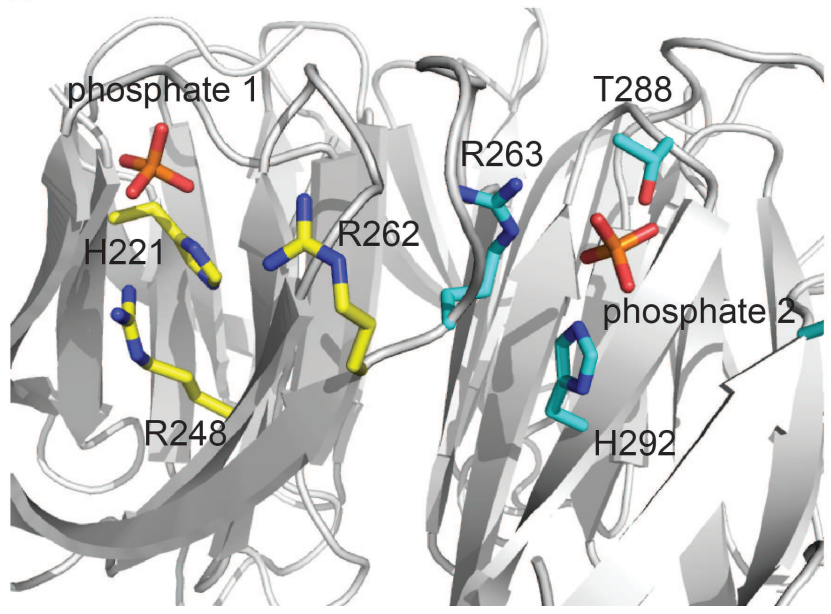

Figure 5.11: Crystal bound ligands for the determined PaAtg18 structures at $1.8 \AA$ resolution (A) and $2.3 \AA$ resolution (B). Important residues in binding pocket 1 are represented in yellow sticks and those in binding pocket 2 in blue sticks.

Crystal bound ligands are found in this pockets, i.e., citrate for binding site 1 and phosphate for binding site 2. All essential residues in the binding pockets have a nicely defined electron density with the exception of K444. When overlaid with the PI bound model of KlHsv2 (Figure 5.12, B), it can be observed that the essential residues in binding pocket 1 are conserved, while in binding pocket 2, instead of the basic residue (K245) binding the $\mathrm{P} 5$ of PIs, one can observe a polar one (T288). A model for the PI(3,5) $\mathrm{P}_{2}$ binding by PaAtg 18 was done based on the ligand docking performed for KlHsv2. In binding pocket 1, H221 makes a salt bridge with P5, R248 with P3 and R262 binds P1. Similarly, in binding pocket 2, salt bridges are made in between P1 and R263 and H292, while P3 binds to K444.

An alignment of the closest PaAtg18 yeast homologs of PaAtg18 was done (Figure 5.13) in order to analyze its surface conservation. There are four surface regions that show higher conservation (Figure 5.10, B). The first two are two pockets on the bottom of the propeller. These pockets are separated by blade 5 , the one containing 
Table 5.8: Diffraction data and refinement statistics for the phosphate bound $2.3 \AA$ selenomethionine labeled PaAtg 18 structure.

\begin{tabular}{|c|c|}
\hline $\begin{array}{r}\text { Space group } \\
\text { Cell dimensions }(\AA)\end{array}$ & $\begin{array}{r}P 1 \\
a=58.3, b=58.3, c=62.3 \\
\alpha=83.7^{\circ}, \beta=80.9^{\circ}, \gamma=86.8^{\circ}\end{array}$ \\
\hline Resolution range $(\AA)$ & $45.55-2.3(2.382-2.3)$ \\
\hline Total reflections & $255465(35132)$ \\
\hline Unique reflections & $35132(3439)$ \\
\hline Multiplicity & 7.3 \\
\hline Completeness $(\%)$ & $98.4(96.8)$ \\
\hline Mean $\mathrm{I} / \sigma$ & $9.9(2.0)$ \\
\hline Wilson B-factor $\left(\AA^{2}\right)$ & 35.5 \\
\hline \multicolumn{2}{|l|}{ Refinement } \\
\hline R-work & $0.25(0.35)$ \\
\hline $\mathrm{R}$-free & $0.29(0.38)$ \\
\hline \multicolumn{2}{|l|}{ No of atoms present } \\
\hline macromolecules & 4660 \\
\hline ligands & 20 \\
\hline water & 60 \\
\hline \multicolumn{2}{|l|}{ RMS } \\
\hline bonds & 0.004 \\
\hline angles & 0.74 \\
\hline \multicolumn{2}{|l|}{ B-factors $\left(\AA^{2}\right)$} \\
\hline overall & 42.8 \\
\hline macromolecules & 42.8 \\
\hline ligands (phosphate, phosphate) & 50.6 \\
\hline solvent & 36.8 \\
\hline \multicolumn{2}{|l|}{ Structure validation } \\
\hline Ramachandran favored (\%) & 97 \\
\hline Ramachandran outliers (\%) & 0 \\
\hline Clashscore & 1.92 \\
\hline
\end{tabular}




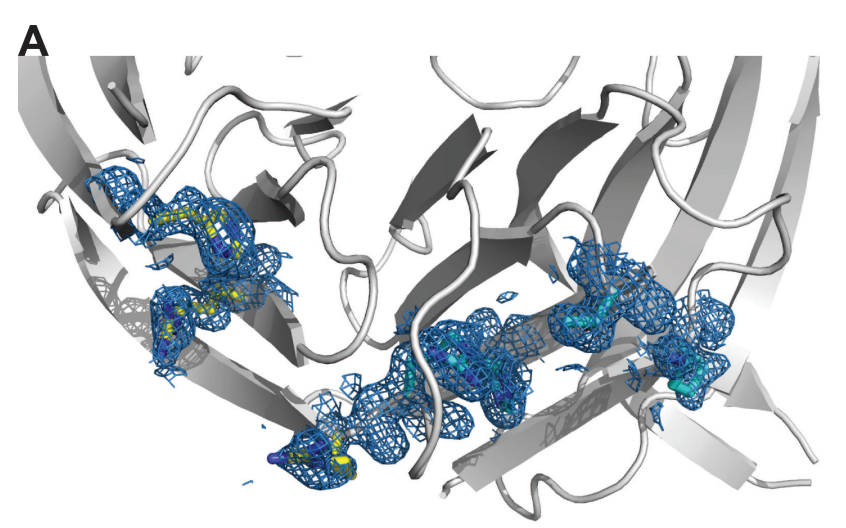

B
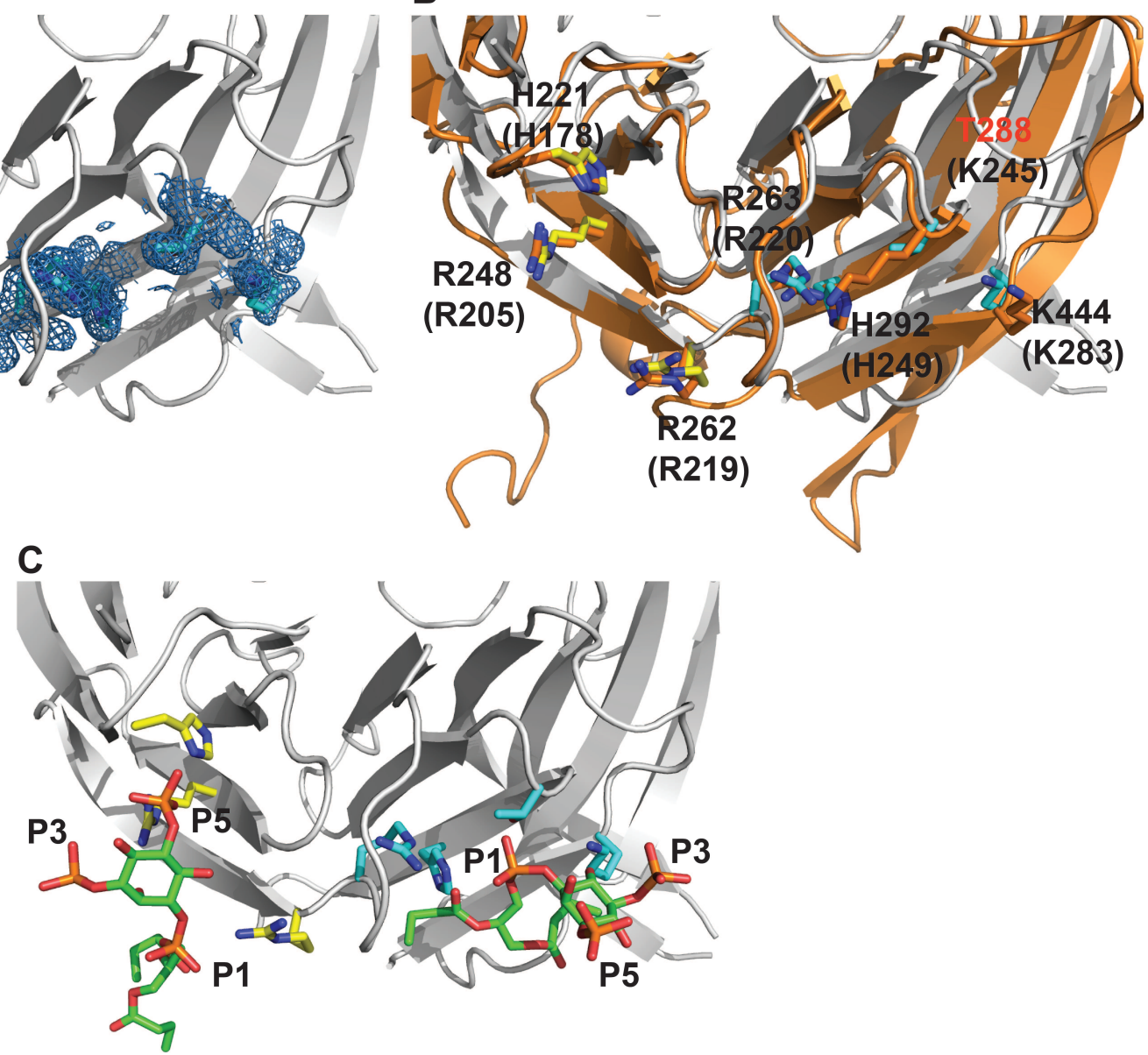

Figure 5.12: PaAtg18 phosphoinositides binding pockets. (A) Close-up of the two phosphoinositide binding sites with the overlaid $1.8 \AA$ resolution $2 \mathrm{mF}_{o}-\mathrm{DF}_{c}$ electron density map contoured at $1.0 \sigma$. (B) Close-up of the phosphoinositide binding sites of the overlaid PaAtg18 and KlHsv2 structures. PaAtg18 is shown in gray with binding site 1 in yellow sticks and binding site 2 in cyan sticks, while KlHsv2 is shown in orange. (C) Model for PaAtg18 PI(3,5) $\mathrm{P}_{2}$ binding based on the computational docking performed for KlHsv2. $\mathrm{PI}(3,5) \mathrm{P}_{2}$ is represented in green sticks. For simplicity, only panel $(\mathrm{B})$ is labeled while $(\mathrm{A})$ and $(\mathrm{C})$ are in the same conformation. 
the FRRG motif. Another conserved surface is present on the rim of the propeller in the vicinity of blades 1 and 2. Another conserved region is localized on the side of the propeller, in blade 4 .

In terms of electrostatic properties of the surface, the two conserved pockets in the vicinity of the FRRG motif are basic (Figure 5.10, C). The conserved surface on the rim of blades 1 and 2, as well as the region conserved in blade 4 , are hydrophobic.

\subsection{Analysis of P. angusta Atg18 membrane binding orientation using fluores- cence measurements}

\subsubsection{Selection of reporter positions for fluorescence labeling}

In section 3.2, I proposed a model for PROPPIN membrane binding based on docking studies of PIs into KlHsv2 and MD simulations of the KlHsv2 membrane system. In order to experimentally test this model, we have chosen to use a fluorescence based method [202]. In short, selected sites are mutated to cysteines and labeled with NBD. The protein should contain no other cysteines. NBD increases its emission fluorescence in a hydrophobic environment. Using this property, one can observe the emission spectra of the NBD labeled protein in solution and upon addition of LUVs. If the labelled site inserts in the membrane or is in proximity of the membrane, then the emission intensity at $531 \mathrm{~nm}$ will increase.

By aligning PaAtg18 with the KlHsv2 in the PROPPIN model for membrane binding, I have obtained the model in Figure 5.14, A. PaAtg18 is oriented with its propeller axis parallel to the membrane surface, while the binding pockets defined by the FRRG motif are in the proximity of the membrane. Some $\beta$-strands are found very close to the membrane, namely $6 \mathrm{C}, 6 \mathrm{D}, 7 \mathrm{~A}$ and $7 \mathrm{~B}$. Based on this model, I have chosen five positions to act as fluorescence reporter. The residues were picked such that two are close to the PI binding pockets (S448 and S459), one is found on the opposite side of the $\beta$-propeller compared to the binding pockets (the wild type C113 was used for this purpose) and two are found in between these two sites (S51 and S157). Moreover, I took care that the chosen residues are surface exposed in order to easily label them with NBD. Before generating these cysteine mutants, I needed a PaAtg18 mutant where all intrinsic cysteined are mutated to serines to allow specific labelling.

\subsubsection{Membrane binding and folding control of the P. angusta Atg18 mutants}

First, the five natural cysteines in PaAtg18 were mutated to serines and then the selected cysteines were then introduced. The mutants were expressed and purified as optimized in section 4.1.3. Next, their $\mathrm{PI}(3,5) \mathrm{P}_{2}$ binding ability was tested in flotation assays using SUVs containing $1 \% \mathrm{PI}(3,5) \mathrm{P}_{2}$. When proteins bind to liposomes they are found in the light top fractions. Figure 5.15, A shows that all mutants were bound to the liposomes indicating that their binding pockets were intact and they could still bind to the membrane. In addition, folding was tested for all mutants through CD measurements and compared to wild type PaAtg 18 . The CD spectra shows the $205 \mathrm{~nm}$ PaAtg 18 minima for all the mutants (Figure 5.15, B and Appendix figure 8.3). The stability of the PaAtg 18 mutants was tested through a CD melting curve taken at $216 \mathrm{~nm}$ from $30^{\circ} \mathrm{C}$ to $80^{\circ} \mathrm{C}$ (Figure 5.15, $\mathrm{C}$ and Appendix figure 8.3). The determined melting temperatures are summarized in Table 5.9 The mutants are approximativelly $8^{\circ} \mathrm{C}$ less stable than wild type PaAtg 18 . 

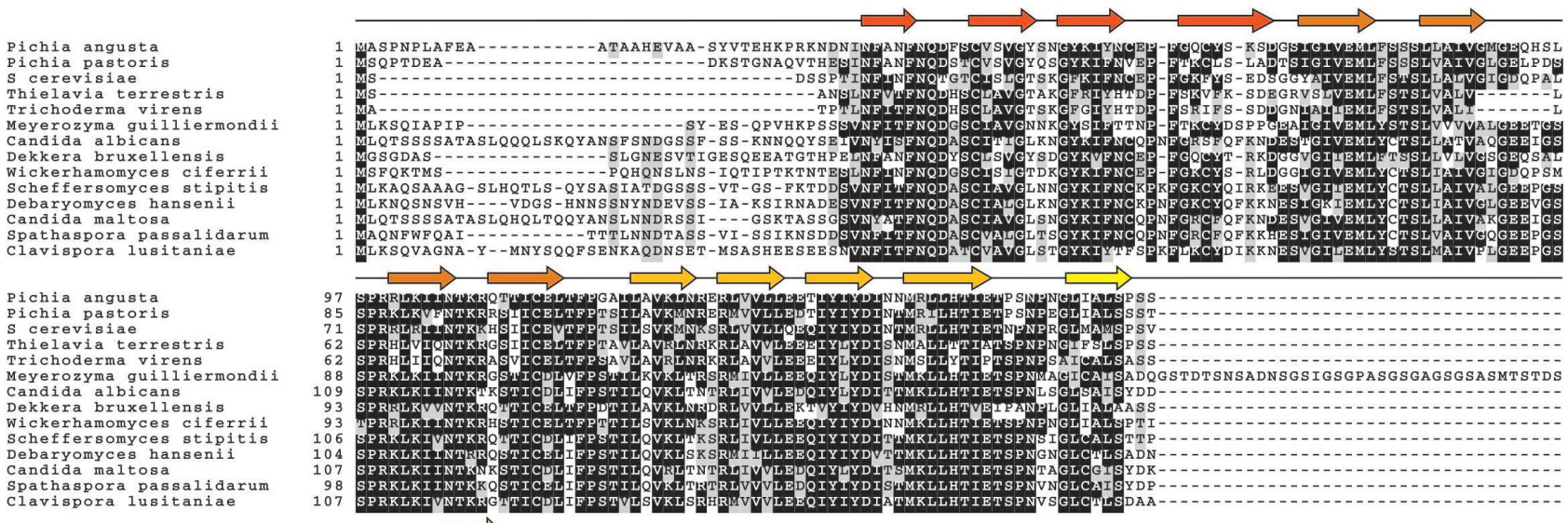

Pichia angusta
Pichia pastoris
scerevisiae
Thielavia terrestris
Trichoderma virens
Meyerozymaguiliermondi
Candida albicans
Dekkerabruxellensis
Wickerhamomyces ciferrii
Scheffersomyces stipitis
Debaryomyces hansenii
Candida maltosa
Spathaspora passalidarum
Clavisporalusitaniae

Pichia angusta
Pichia pastoris
Scerevisiae

S cerevisiae
Thielavia terrestris

Meyerozyma guilliermondi

Candida albicans

Wickerhamomyces ciferrii

Debaryomyces hansenii

Candida maltosa
Cpathaspora passalidarum
clavpora lusitaniae

Pichia angusta
Pichia pastoris
Scerevisiae

S cerevisiae
Thielavia terrestris

Meyerozyma guilliermondi

Candida albicans
Dekkera bruxellensis

Wickerhamomyces ciferrii

Candida maltosa

Spathaspora passalidarum

Pichia angusta

s cerevisiae
Thielavia terrestris

Trichoderma virens

Candida albicans Atg18
Dekkera bruxellensis

Wickerhamomyces ciferri

Debaryomyces has

Spathaspora passalidarum
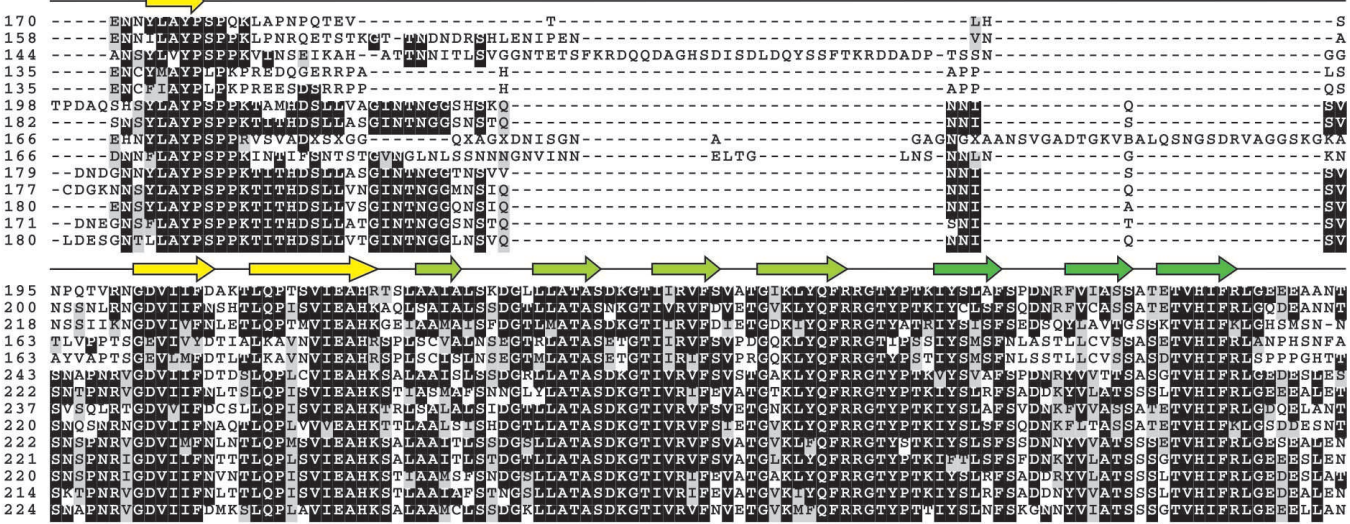

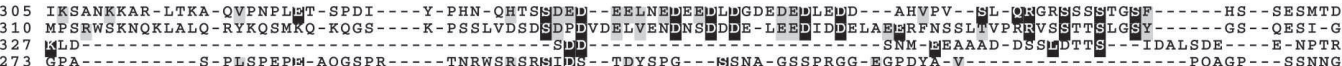
327
273
GLDA-

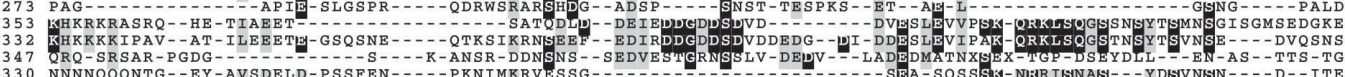

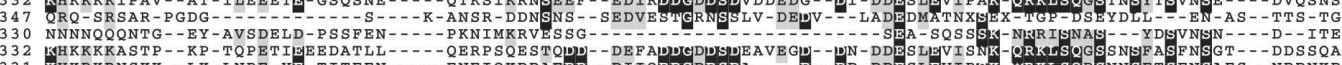

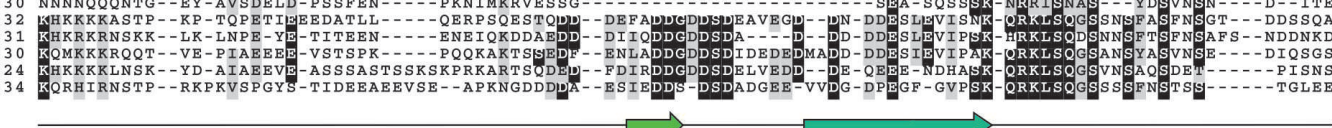

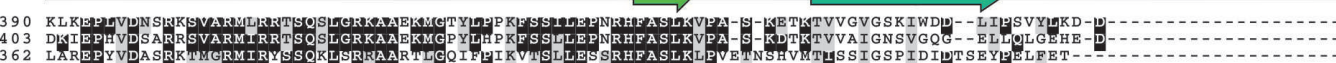

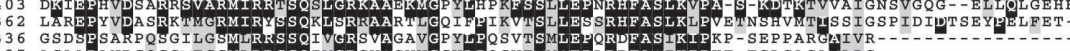

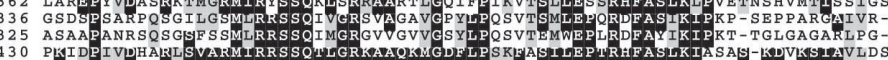

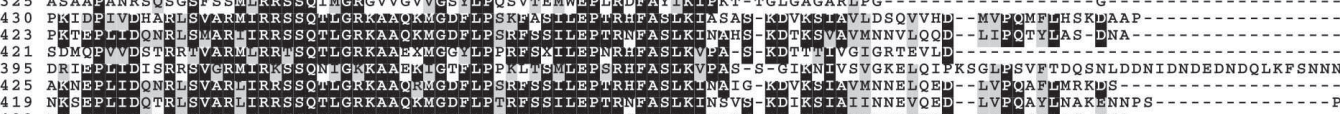

425
AKNEPLIDQNRL SVARLIRRSSQ

Pichia angusta

Scerevisiae

Mrichoderma virens

Candida albicans

Wickerhamomyces ciferrii
Scheffersomyces stipitis

Candida maltosa

Spathaspora passalidarum

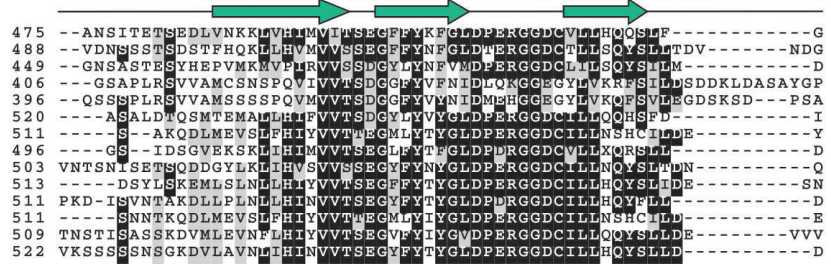

Figure 5.13: Multiple sequence alignment of yeast Atg18 homologs.

PaAtg18 is aligned to its close homologs from Pichia pastoris, S. cerevisiae, Thielavia terrestris, Trichoderma virens, Meyerozyma guilliermondii, Candida albicans, Dekkera bruxellensis, Wickerhamomyces ciferrii, Scheffersomyces stipites, Debaryomyces hansenii, Candida maltosa, Spathaspora passalidarum and Clavispora lusitaniae using the T-Coffee [185] server. Secondary structure elements are shown based on the structure of PaAtg18. 
A

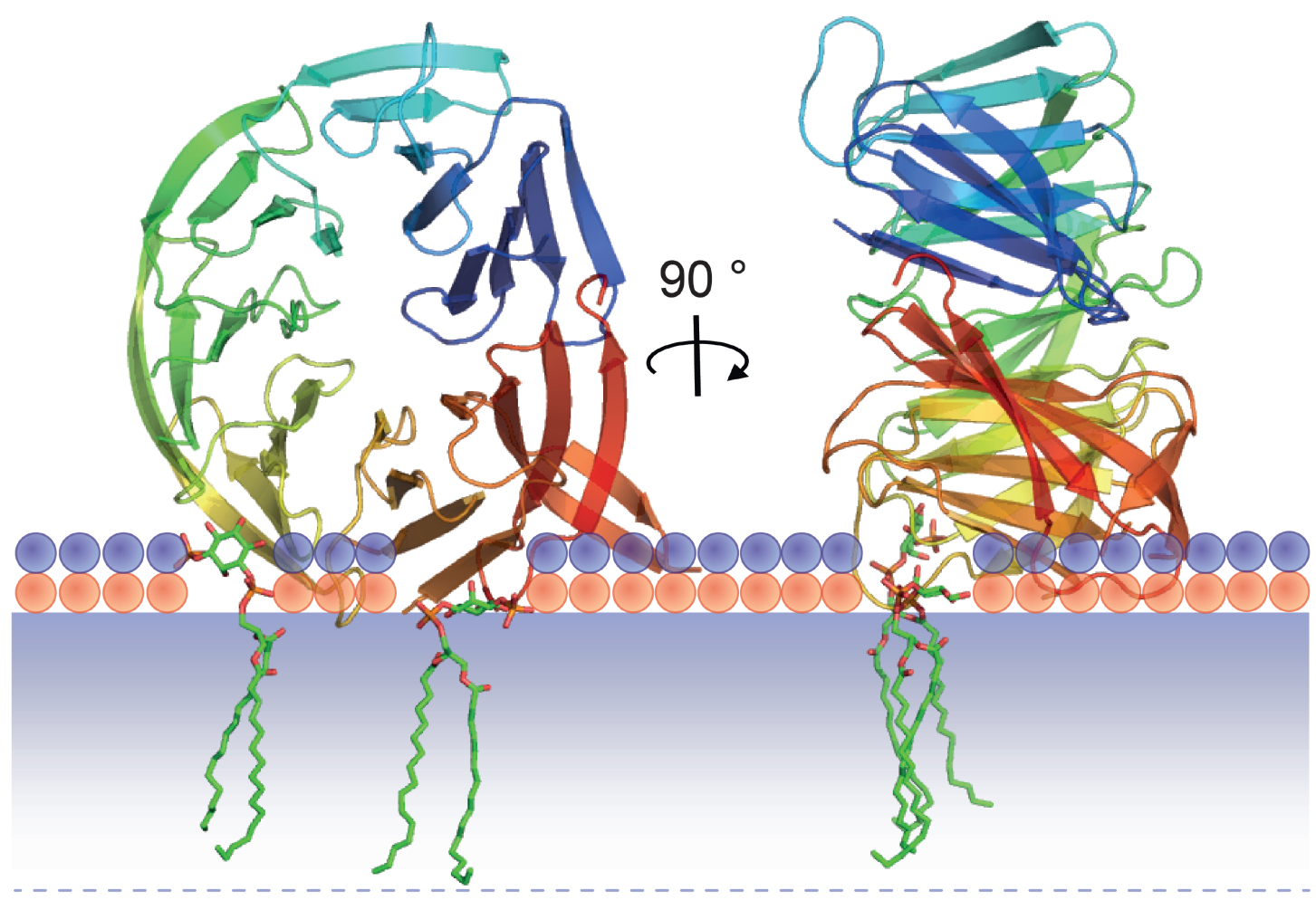

B

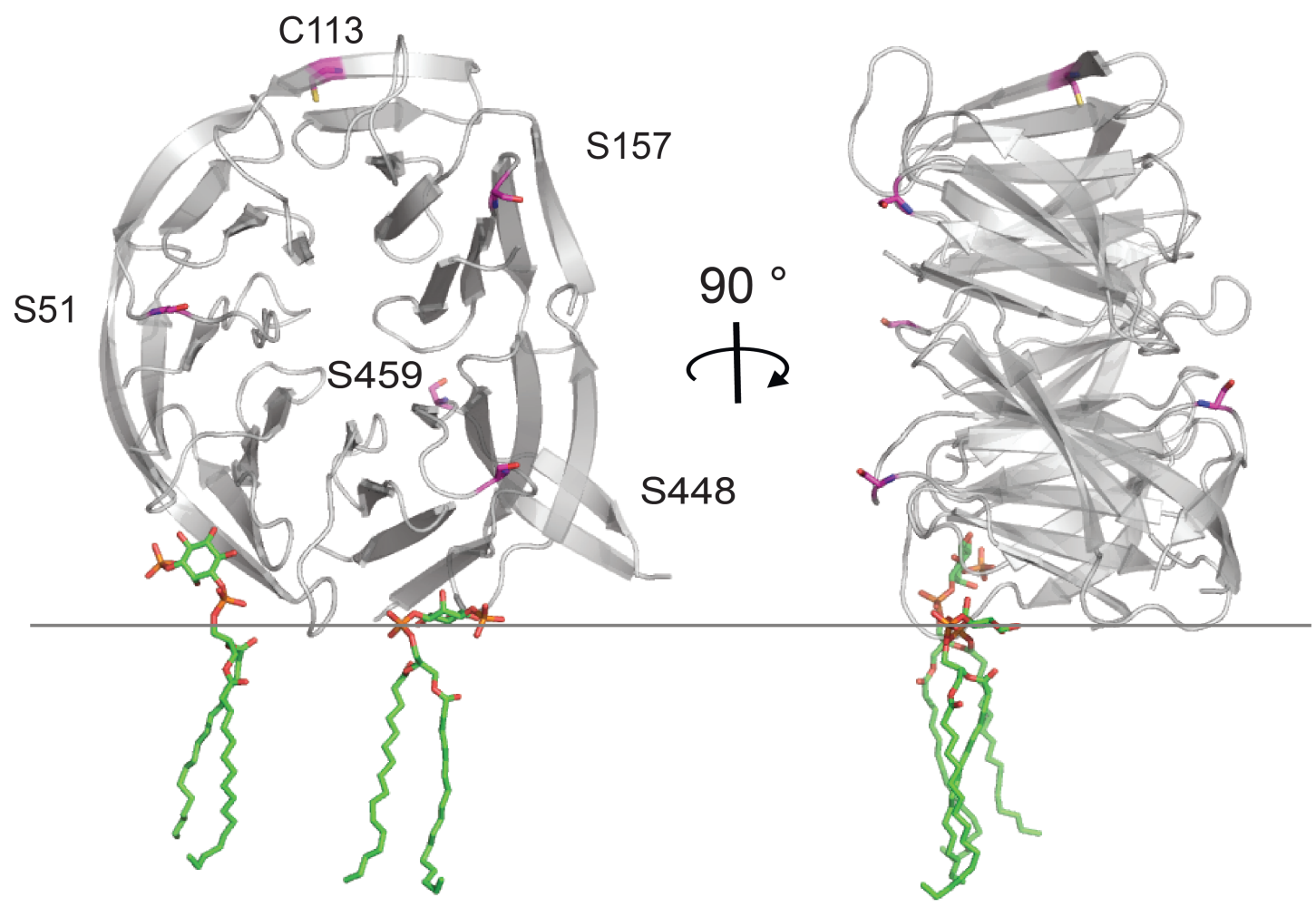

Figure 5.14: Computational model for PaAtg18 membrane binding. (A) A computational model for PaAtg18 membrane binding was done based on the docking studies of PIs into KlHsv2. The two PI3Ps are shown in green sticks. The positions of the phosphates of the membrane phospholipids are shown through orange circles, while the blue circles represent the polar groups of these phospholipids. The shaded violet bar depicts the fatty acid tails of the phospholipids from the cytosolic leaflet of the bilayer. The dashed blue line represents the center of the bilayer. (B) Selection of reporter positions for fluorescence labeling. For reasons of simplicity, the lipid bilayer is shown through a gray dashed line. 
A

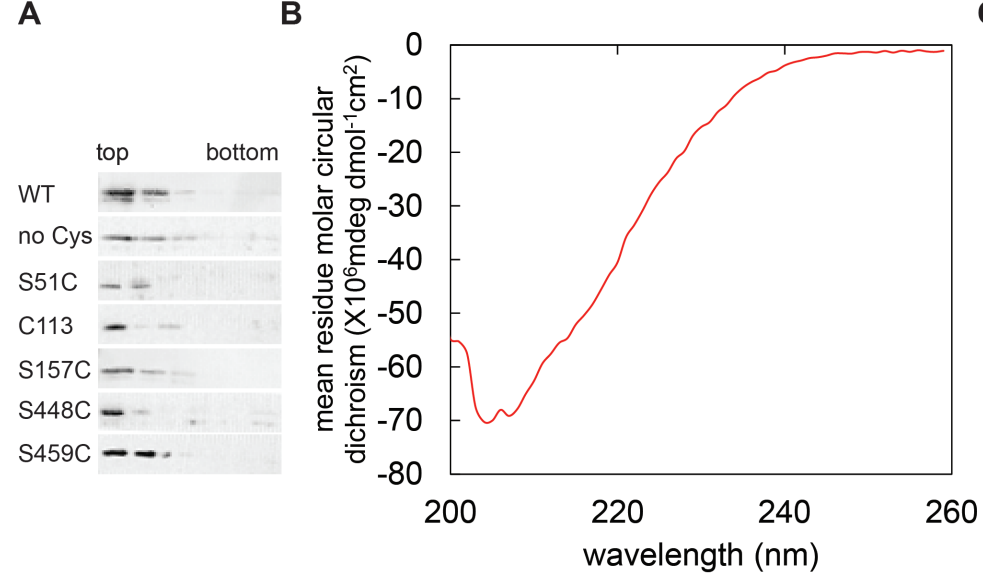

C

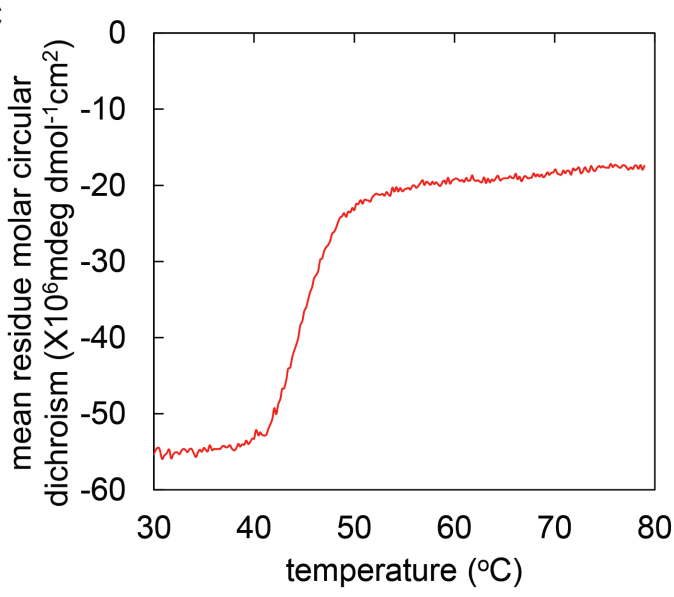

Figure 5.15: Binding and folding control of single cysteine PaAtg18 mutants. (A) Liposome flotation assays of PaAtg18 single cysteine mutants with SUVs consisting of PC:PE:Texas-Red-PE:PI(3,5)P $\mathrm{P}_{2}$ (74:23:2:1, weight ratio). (B) CD spectrum of PaAtg18 no cysteine mutant from $260 \mathrm{~nm}$ to $200 \mathrm{~nm}$. (C) Melting curve for PaAtg 18 no cysteine mutant was taken at $216 \mathrm{~nm}$ from $30^{\circ} \mathrm{C}$ to $80^{\circ} \mathrm{C}$.

Table 5.9: Summary of the melting temperatures of the wild type PaAtg18 compared to the single cysteine binding mutants

\begin{tabular}{rcc}
\hline & $\mathbf{T}_{m}\left({ }^{\circ} \mathbf{C}\right)$ & Fitting error $\left({ }^{\circ} \mathbf{C}\right)$ \\
\hline wild type & 53.41 & 0.06 \\
no cys PaAtg18) & 45.15 & 0.05 \\
PaAtg18(S51C) & 44.95 & 0.10 \\
PaAtt18(C113) & 44.07 & 0.04 \\
PaAtg18(S157C) & 45.17 & 0.09 \\
PaAtg18(S448C) & 45.36 & 0.08 \\
PaAtg18(S459C) & 44.95 & 0.04 \\
\hline
\end{tabular}




\subsubsection{P. angusta Atg18 membrane binding orientation}

The purified PaAtg18 single cysteine mutants were labeled with NBD. For preliminary measurement, PaAtg18(S448C) and PaAtg18(C113) were used. Their fluorescence spectra was measured upon excitation at $478 \mathrm{~nm}$ in the absence and presence of LUVs containing $1 \% \mathrm{PI}(3,5) \mathrm{P}_{2}$ (Figure $5.16 \mathrm{~A}$ and $\mathrm{B}$ ). The increase in emission at $531 \mathrm{~nm}$ upon LUVs addition was quantified (Figure 5.16 C). The difference in emission at 531 $\mathrm{nm}$ is higher for PaAtg18(S448C) than for PaAtg18(C113), indicating that S448 is closer to the membrane than $\mathrm{C} 113$ is, which is consistent with the proposed model.
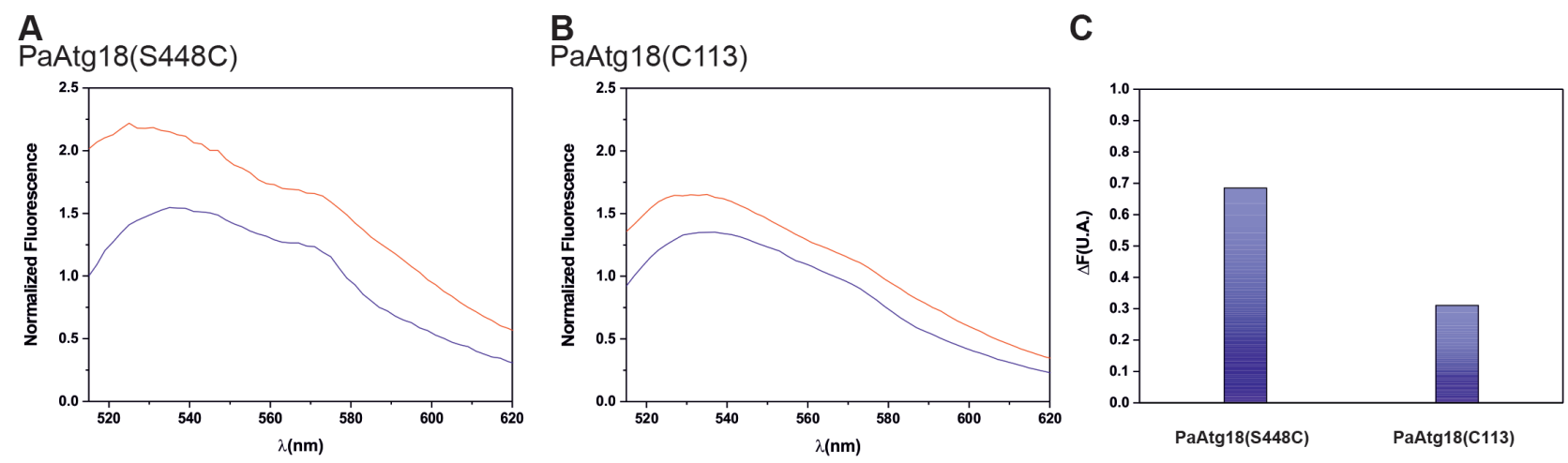

Figure 5.16: Analysis of PaAtg18 membrane binding orientation through fluorescence measurements.. Emission spectra of NBD labeled PaAtg18(S448C) (A) and PaAtg18(C113) (B) free in solution (blue curve) and upon LUVs addition (red curve). LUVs consisted of PC:PE:PI(3,5) $\mathrm{P}_{2}$ (74:24:2:, weight ratio). (C) Quantification of emission increase upon LUVs addition at $531 \mathrm{~nm}$. Experiments were performed in collaboration with Dr. Ángel Lara Perez. 


\section{Expression and purification of PROPPINs binding partners}

\subsection{The Atg2-Atg18 complex}

\subsubsection{The S. cerevisiae Atg2}

In macroautophagy, the most important interaction partner of Atg18 is Atg2 [79]. Together they form Atg2Atg18 complex, which is essential for autophagosomal membrane growth and autophagosome formation. Most of the in vivo studies on Atg2 were done in S. cerevisiae [136, 135, 137, 79, 140, 2]. Only in the recent years the human Atg2 [144, 143, 141] started being investigated. However, it is not well characterized which of the WIPI proteins - human Atg18 homologs - are the Atg2 interaction partner. Interestingly, the human Atg2A interacts with WIPI4 [32], which is the human homolog of Hsv2 [96]. Taking into consideration that the functionality and interaction of the yeast Atg2-Atg18 complex is well characterized and that I determined the structure of an yeast Atg18, the next crystallization target was the S. cerevisiae Atg2-Atg18 complex. This section summarizes experiments done towards the optimization of S. cerevisiae Atg2 expression in S. cerevisiae as full lenght and in E. coli as N-terminal subdomains.

\subsubsection{S. cerevisiae Atg2 expression optimization in S. cerevisiae}

As a first step towards the structural characterization of the S. cerevisiae Atg2-Atg18, the expression and purification of S. cerevisiae Atg2 (ScAtg2) alone was tried. In 2005, a moveable ORF library of 5854 yeast expression plasmids was constructed

Keeping in mind that the expression might be low, I have tested two expression media for S. cerivisiae: $2 \%$ galactose, $2 \%$ raffinose, the standard condition for moderate induction; and $2 \%$ galactose $+3 \%$ glycerol $+2 \%$ ethanol, for a strong transcription induction (Figure 6.1). Samples were collected over a 29 hours time course, cells were opened using glass beads and both the whole cell lysate and the soluble fractions were analyzed on Western blots. Proteins were detected using an anti-HA polyclonal antibody. As schematically represented in Figure 6.2, the fusion protein product of the expression will have a C-terminal HA-tag. Hence, only the C-terminal degradation fragments and the full-length protein will be detected in Western blot analysis. For both expression media used, it can be observed that longer expression times lead to more degradation products. The protein bands that can be clearly distinguished have an approximate size of: $60 \mathrm{kDa}, 80 \mathrm{kDa}, 100 \mathrm{kDa}$ and above $170 \mathrm{kDa}$ - most probably, the full length fusion construct, expected at $197 \mathrm{kDa}$. In the case of the $2 \%$ 
ScAtg2

A

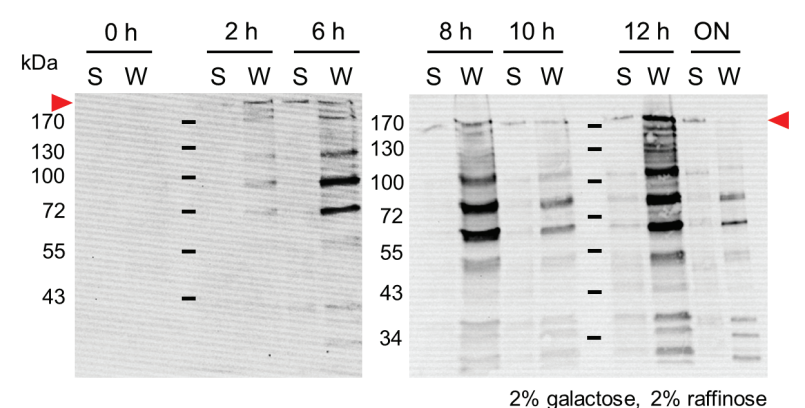

B

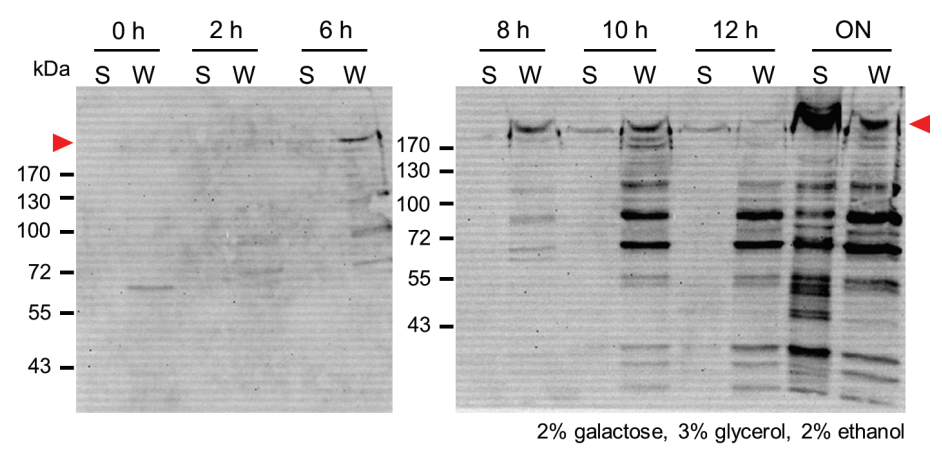

Figure 6.1: Expression optimization for ScAtg2 in S. cerevisiae.

Western blots representing the optimization steps for the expression of ScAtg2. Detection is done with an anti-HA polyclonal antibody. Uninduced sample is taken as 0 hours. Red arrows indicate where the ScAtg2 full length protein would be expected. An expression time course is observed for two expression media: $2 \%$ galactose $+2 \%$ raffinose (A) and $2 \%$ galactose $+3 \%$ galactose $+2 \%$ ethanol (B). $\mathrm{S}$ - soluble fraction; W - whole cell lysate; ON - overnight.

galactose, $2 \%$ raffinose media, after 6 hours after induction, the level of the full length soluble protein stays the same, while in the strong inducing media, the level of full length protein product increases in time.

Next, I have tried to purify ScAtg2 in S. cerevisiae using the $2 \%$ galactose $+3 \%$ glycerol $+2 \%$ ethanol media. Since in Figure 6.1, B, it can be observed that after 6 hours of expression, the amount of degradation products is low, I have chosen this condition. After the expression, cells were harvested, opened and the soluble fraction was loaded to a $1 \mathrm{~mL}$ HisTrap affinity column. After loading and wash of the unbound protein, a gradient of imidazole from $20 \mathrm{mM}$ to $500 \mathrm{mM}$ was used for ScAtg2 elution. The purification fractions were analyzed both by SDS-PAGE (Figure 6.2, A) and Western blot analysis Figure 6.2, B. Full length protein, but also degradation fragments appear in all fractions.

In order to remove the degradation fragments and obtain a pure sample, I have tried imidazole gradient optimization for the affinity purification step, separation of fragments in a subsequent ion exchange - both anion and cation exchange were tried with the appropriate buffer- and ammonium sulfate precipitation. None of these led to a pure ScAtg2 sample. The smaller protein bands from the SDS-PAGE gels were confirmed to be ScAtg2 through mass spectrometry peptide analysis.

\subsubsection{S. cerevisiae Atg2 domains expression optimization in E. coli}

Since the expression in S. cerevisiae has proven problematic, we decided to try expression in E. coli. The molecular weight of full length ScAtg2 is $178 \mathrm{kDa}$, which is too large for bacterial expression. This is why, 


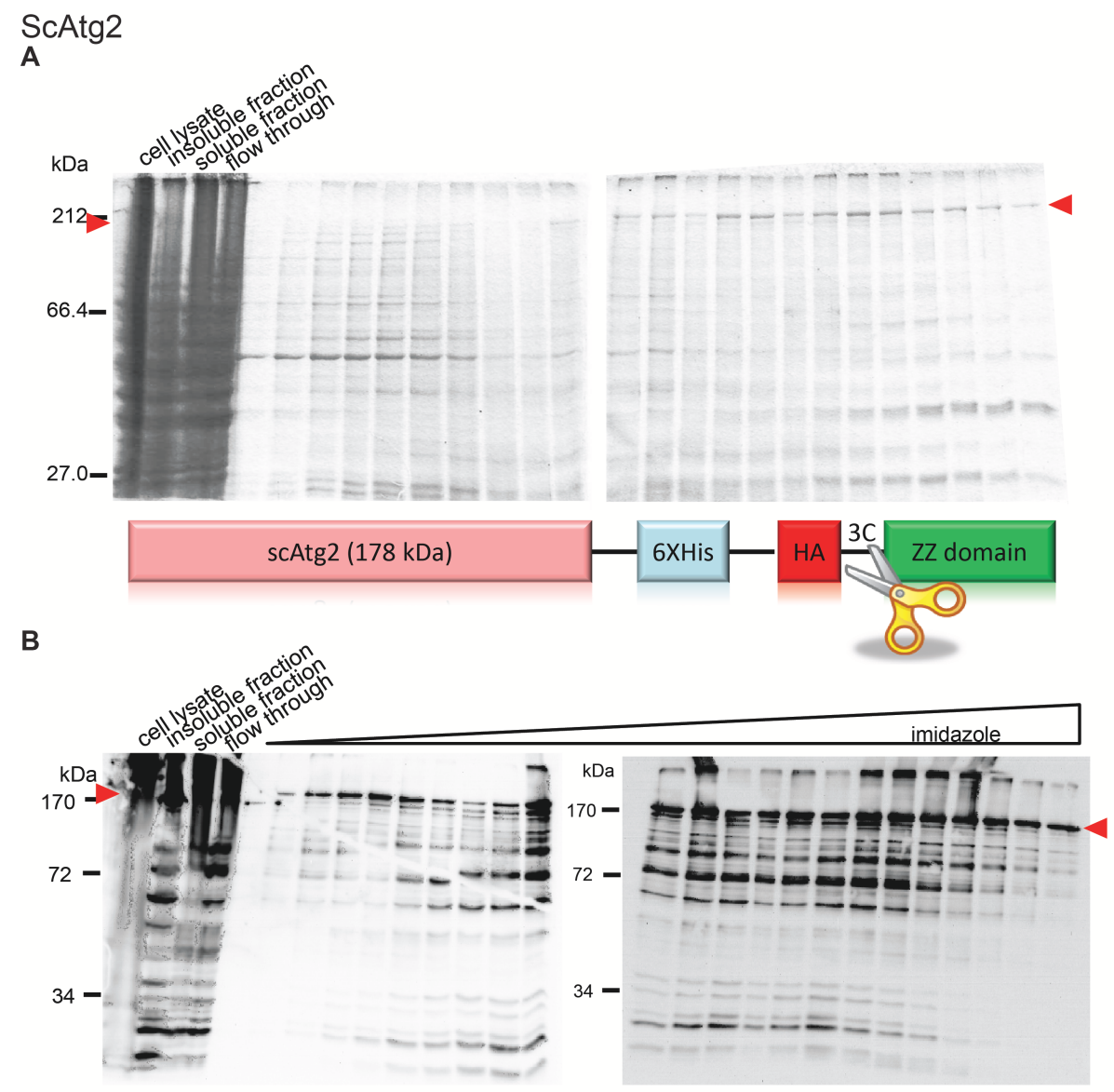

Figure 6.2: Affinity purification of ScAtg2 from S. cerevisiae

ScAtg2 was expressed in S. cerevisiae using a media having galactose, glycerol and ethanol as carbon source. Cells were harvested 6 hours after induction, opened and the soluble fraction was loaded on a $1 \mathrm{~mL}$ HisTrap. The bound protein was eluted with an imidazole gradient and fractions were analyzed using SDS-PAGE (A) and Western blot (B).

together with Sebastian David, a Master student, I tried to express N-terminal fragments of ScAtg2 as this part of the protein is known to interact with $\operatorname{Atg} 18$ [141].

The expression vector chosen was pET-28a(+). The gene transcription in this vector is under the control of the $T 7$ promoter and the protein will be expressed as a fusion construct having a His-tag at the N-terminal. The Jpred server [203] was used to choose constructs such that the secondary features of ScAtg2 are not disrupted. Eight N-terminal fragments of the ScAtg2 gene were successfully cloned. The expected protein products are ScAtg2(1-161), ScAtg2(1-157), ScAtg2(1-189), ScAtg2(1-229), ScAtg2(1-289), ScAtg2(1-307), ScAtg2(1-327) and ScAtg2(1-354). The expression of the constructs was tested in TB media with $0.1 \mathrm{mM}$ IPTG induction at $25^{\circ} \mathrm{C}$. Samples were collected before induction, at $3 \mathrm{~h}$ post induction and from overnight expression. Whole cell extract, soluble fractions, $\mathrm{Ni}^{2+}$-sepharose enriched soluble fractions were analyzed on SDS-PAGE gels (Figure 6.3). None of the constructs yielded soluble protein. In the affinity beads enriched samples, three bands can be observed for all constructs: $30 \mathrm{kDa}, 40 \mathrm{kDa}$ and $70 \mathrm{kDa}$. Nevertheless, the purification of these constructs was tried, and as expected, no soluble ScAtg2 domain was obtain. 


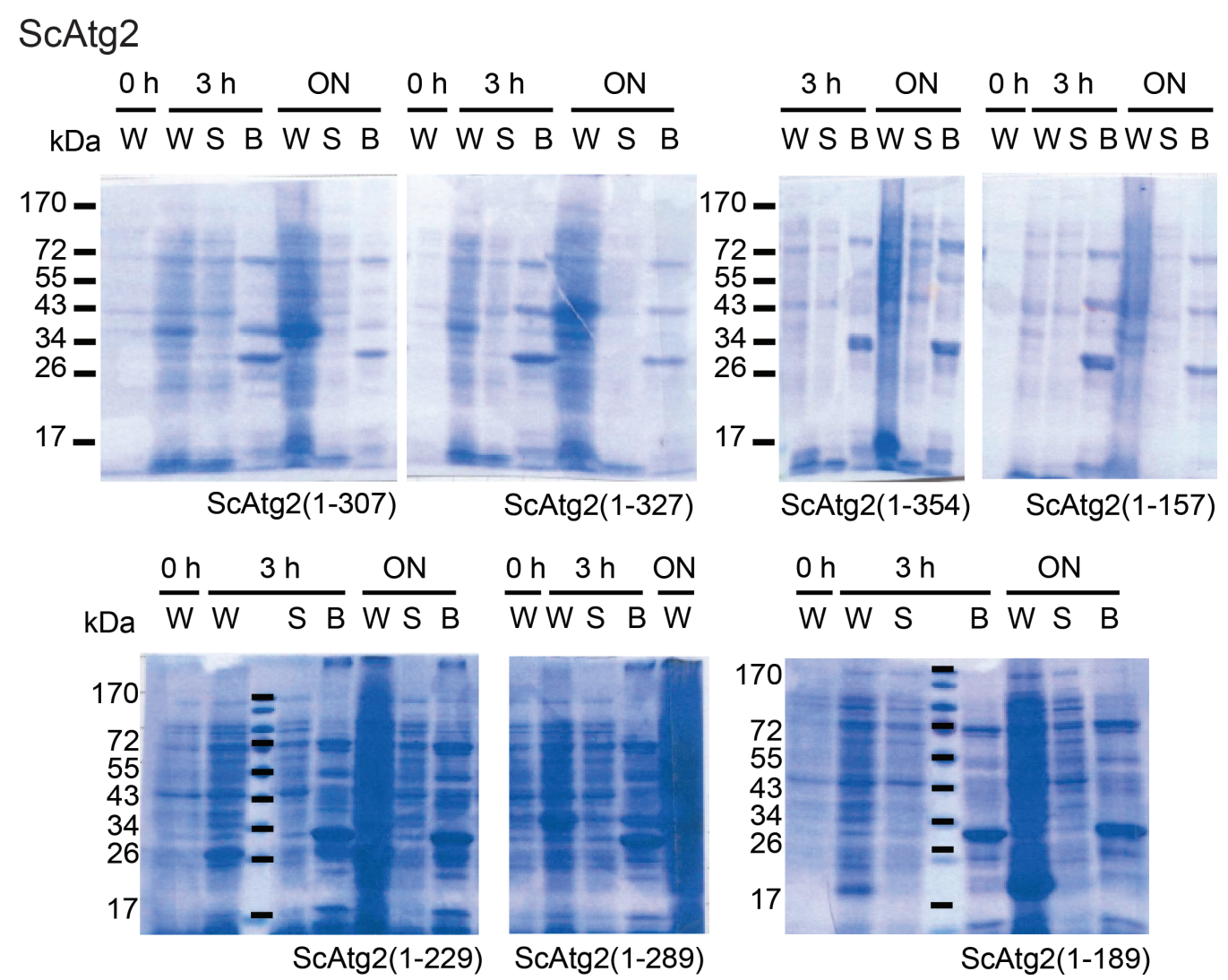

Figure 6.3: Expression optimization for ScAtg2 N-terminal fragments in E. coli.

SDS-PAGE gels showing the protein content of the expression samples harvested at different time points for different constructs. Expression media used was TB induced with $0.1 \mathrm{mM}$ IPTG at $25^{\circ} \mathrm{C}$. Uninduced sample is represented as 0 hours. Samples were also harvested at 3 hours after induction and after overnight expression. W - whole cell lysate; S - soluble fraction; B - soluble fraction enriched on $\mathrm{Ni}^{2+}$-NTA beads; ON - overnight.

\subsubsection{C. thermophilum Atg2 domains expression optimization in E. coli}

Since the expression of ScAtg2 both in S. cerevisiae, as full length and in E. coli as N-terminal domains was proven problematic, I moved up to evolutionary ladder and chose as crystallization target the $C$. thermophilum Atg2. C. thermophilum is a filamentous fungus having an optimal growth temperature of approximatively $60^{\circ} \mathrm{C}$. Its thermophilic character suggests that its proteins might be more stable therefore easier to crystallize. C. thermophilum genome was recently sequenced [195]. C. thermophilum $\operatorname{Atg} 2(\mathrm{CtA} \operatorname{tg} 2)$ has a $14.4 \%$ identity to ScAtg2.

The molecular weight of full length length ctAtg2 is $226.8 \mathrm{kDa}$ which exceeds the capacity of protein expression in bacterial systems. This is why, together with my Master student, Oleksandr Yagensky, I have tried to express the most conserved part of ctAtg2 - its N-terminal 1-168 fragment. In S. cerevisiae, the Nterminal part of Atg2 was shown to interact with Atg18 [141]. However, there is no proof on this interaction in C. thermophilum or any other organism.

When the N-terminal fragment was chosen, the secondary structure prediction with the help of Jpred server [203] was taken into consideration and no secondary structure element is interrupted, while the C-terminal of CtAtg2(1-168) is found within a loop. The DNA sequence of this N-terminal domain was PCR amplified for the CtAtg2 synthetic gene. This synthetic gene was codon optimized for both bacterial and insect cells 
CtAtg2(1-168)
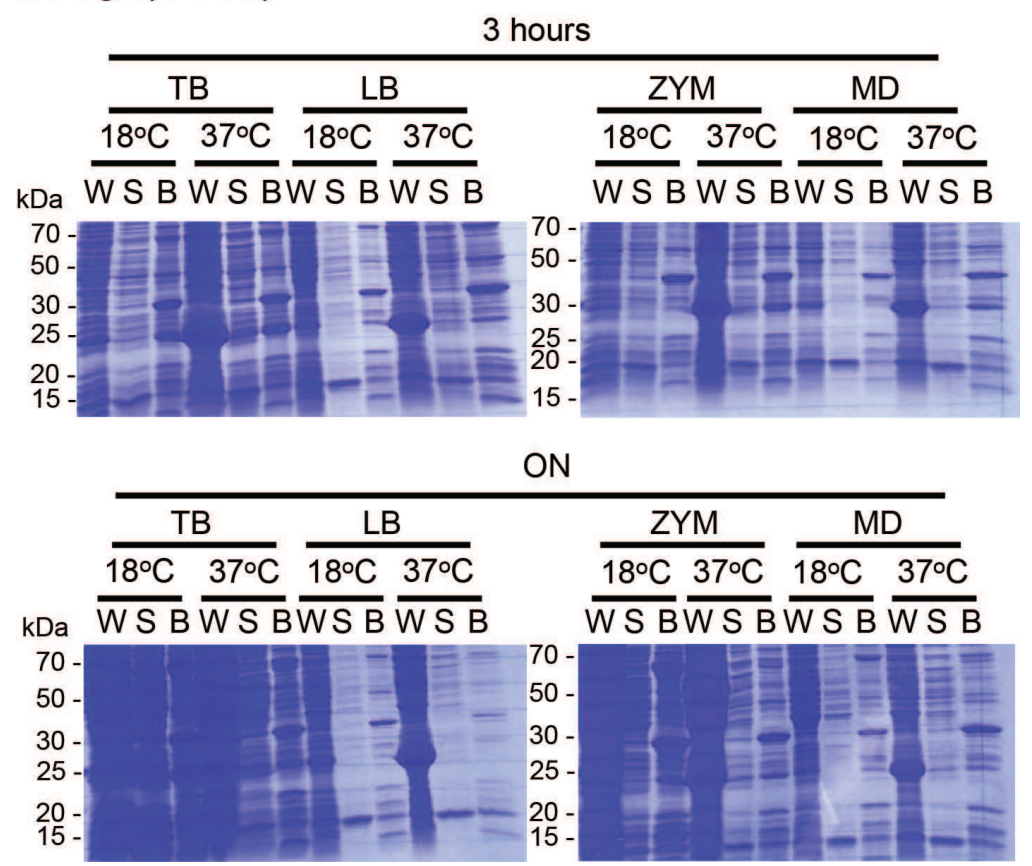

Figure 6.4: Expression optimization for CtAtg2(1-168) in E. coli.

SDS-PAGE gels showing the protein content of the expression samples harvested at different time points for different constructs. Expression media tested were TB and LB induced with $1 \mathrm{mM}$ IPTG and autoinducing media ZYM5052 (ZYM) and MD5052 (MD). Expression was analyzed for both $18^{\circ} \mathrm{C}$ and $37^{\circ} \mathrm{C}$. The expression vector used was pAce1-N-His. Samples were harvested at 3 hours after induction and after overnight expression. W - whole cell lysate; $\mathrm{S}$ - soluble fraction; B - soluble fraction enriched on $\mathrm{Ni}^{2+}-\mathrm{NTA}$ beads; ON - overnight. 
expression. The CtAtg2(1-168) PCR product was cloned in the pAce1-N-His expression vector. An expression test was performed to determine the optimum expression condition for CtAtg2(1-168) in E. coli BL21(DE3). Several expression media and temperatures were tested: auto-inducing media (MD5052 and ZYM5052) and media inducible by the addition of IPTG (LB and TB) at $18^{\circ} \mathrm{C}$ and $37^{\circ} \mathrm{C}$. Samples were taken at 3 hours after induction and after overnight expression. Whole cell extract, soluble fractions and $\mathrm{Ni}^{2+}$-sepharose enriched soluble fractions were analyzed on SDS-PAGE gels (Figure 6.4). The expected size of CtAtg2(1-168) is 20.6 $\mathrm{kDa}$.On the SDS-PAGE gels, an enrichment of a $20.6 \mathrm{kDa}$ protein cannot be observed for samples treated with $\mathrm{Ni}^{2+}$-sepharose beads. However, a strong protein band with an apparent size of $25 \mathrm{kDa}$ can be seen in the whole cell lysate sample but not in the soluble fraction. This might be our protein. In spite of an unsuccessful expression optimization, the purification of ScAtg2(1-168) was tried from cells induced with $1 \mathrm{mM}$ IPTG in TB media overnight at low temperature. However, even though a protein was obtained after purification, an mass spectrometry analysis proved it not to be CtAtg2(1-168) but a bacterial contaminant.
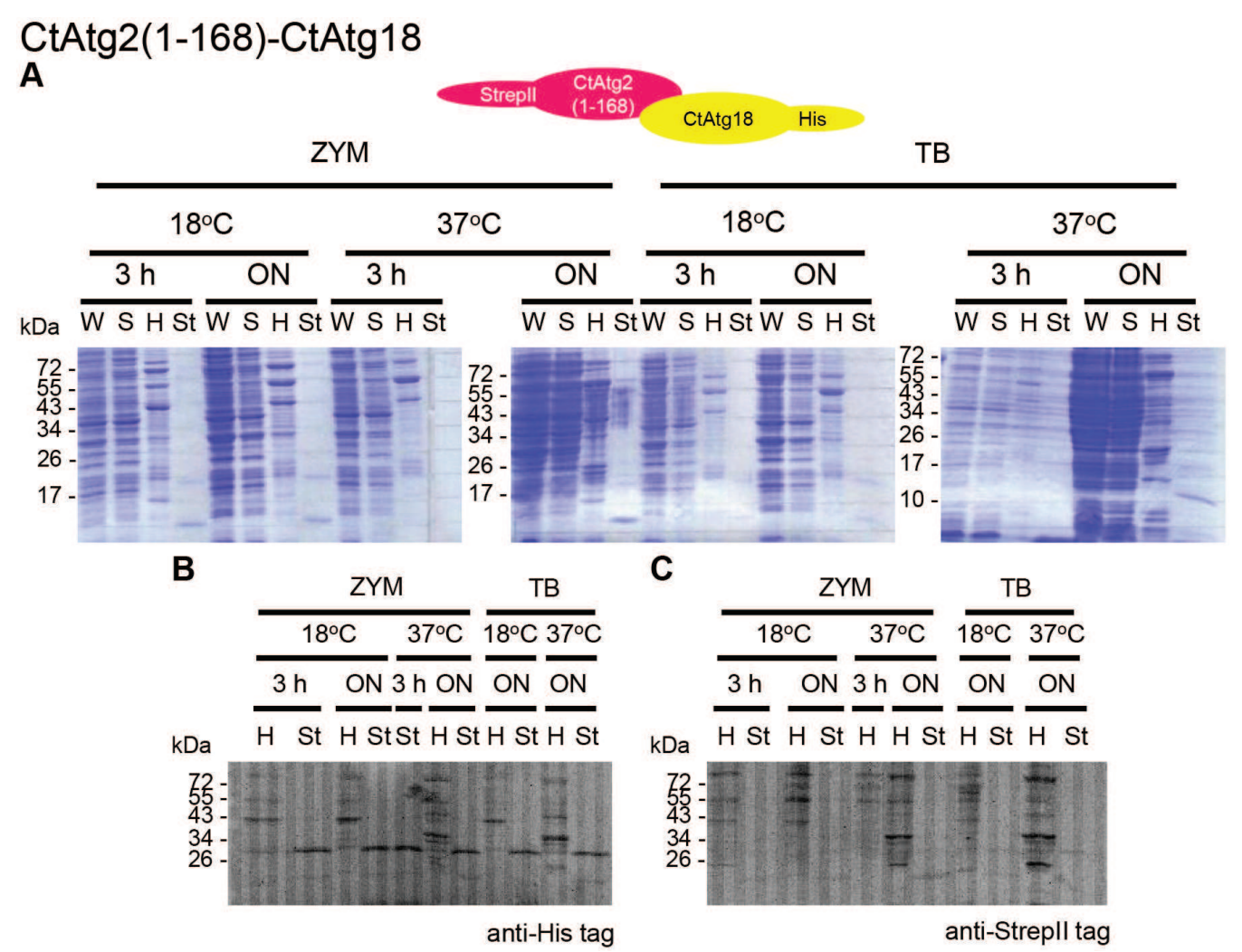

Figure 6.5: Expression optimization for CtAtg2(1-168)-CtAtg18 in E. coli.

Expression media tested were TB induced with $1 \mathrm{mM}$ IPTG and autoinducing media ZYM5052 (ZYM). Expression was analyzed for both $18^{\circ} \mathrm{C}$ and $37^{\circ} \mathrm{C}$. The expression vector used was pAce1-N-His for CtAtg 18 which recombined with pDk-N-StrepII containing the gene for CtAtg2(1-168). Samples were harvested at 3 hours after induction and after overnight expression. W - whole cell lysate; $\mathrm{S}$ - soluble fraction; $\mathrm{H}$ - soluble fraction enriched on $\mathrm{Ni}^{2+}$-NTA beads; $\mathrm{St}$ - soluble fraction enriched on Strep-Tactin beads; ON overnight. Samples collected during the optimization step were analyzed through SDS-PAGE (A), or Western blot with a monoclonal anti-His tag antibody (B) or a monoclonal anti-StepII tag antibody (C).

Some proteins are stabilized by their interaction partner(s). This is used in the stable co-expression of the complex. This is why, next, we have tried to co-express the CtAtg2(1-168)-CtAtg18 complex in E. coli BL21(DE3). CtAtg2(1-168) was cloned in the donor vector of the ACEMBL system, pDk-N-StrepII. This allowed recombination with the previously constructed pAce1-N-His-CtAtg18 (4.1.6). The resulting plasmid 
was used for the complex co-expression optimization in E. coli BL21(DE3). Auto-inducing media, ZYM5052 and TB inducible by the addition of $1 \mathrm{mM}$ IPTG at $18^{\circ} \mathrm{C}$ and $37^{\circ} \mathrm{C}$ were tested. Samples were taken at 3 hours after induction and after overnight expression. Whole cell extract, soluble fractions, $\mathrm{Ni}^{2+}$-sepharose and StrepTactin enriched soluble fractions were analyzed on SDS-PAGE gels (Figure 6.4, A) and Western blots with the detection of the His-tag (Figure 6.4, A) and StrepII-tag (Figure 6.4, C). Neither in the SDS-PAGE analysis, nor in Western blot detection, the expected $20.6 \mathrm{kDa}$, for CtAtg2(1-168) or $50 \mathrm{kDa}$, for CtAtg18 protein bands were observed.

Next, longer N-terminal domains of CtAtg2 were constructed and cloned in the pBADM-41(+) expression vector. The gene transcription in this vector is under the control of the araBAD promoter, which is a tighter promoter that the previously used $T 7$ promoter from the ACEMBL expression vectors [194]. Moreover, from this vector, the protein will be expressed as a fusion construct having MBP at the N-terminal. MBP was reported in helping protein expression by promoting folding [196]. This was done together with Sebastian David, a laboratory rotation Master student.

The Jpred server [203] was used to choose constructs such that the secondary features of CtAtg2 are not disrupted. Nine $\mathrm{N}$-terminal fragments of $\mathrm{CtAtg} 2$ gene were successfully cloned. The expected protein products are MBP-CtAtg2(1-161), MBP-CtAtg2(1-168), MBP-CtAtg2(1-183), MBP-CtAtg2(1-206), MBP-CtAtg2(1-218), MBP-CtAtg2(1-244), MBP-CtAtg2(1-266), MBP-CtAtg2(1-270) and MBP-CtAtg2(1-279). The expression of the constructs was tested in TB media with $1 \mathrm{mg} / \mathrm{L}$ arabinose induction at $25^{\circ} \mathrm{C}$. Samples were collected before induction, at $3 \mathrm{~h}$ post induction and from overnight expression. Whole cell extract, soluble fractions, $\mathrm{Ni}^{2+}$ sepharose enriched soluble fractions were analyzed on SDS-PAGE gels (Figure 6.6). It can be observed that all constructs are highly expressed in a soluble form.

Since the expression test yielded promising results, the purification of MBP-CtAtg2(1-279) was tried. Expression was done in $E$. coli $\mathrm{BL} 21(\mathrm{DE} 3)$ cells grown in TB media with $1 \mathrm{mg} / \mathrm{L}$ arabinose overnight induction at $25^{\circ} \mathrm{C}$. The expression scale was $6.6 \mathrm{~L}$. Cells were harvested and opened, then, the cell lysate loaded on a 5 $\mathrm{mL}$ HisTrap column. The MBP-fused protein was eluted with a two step imidazole gradient from $20 \mathrm{mM}$ to $500 \mathrm{mM}$. The purification chromatogram and the SDS-PAGE sample analysis for this step can be observed in Figure 6.7. A and B. The eluted protein was dialyzed overnight for imidazole and MBP-tag removal using TEV (tobacco etch virus) protease, purified by us in our laboratory. The dialysis was followed by another affinity chromatography using a HisTrap column as shown in Figure 6.7 C.

The SDS-PAGE analysis of the samples show that the protease did not cleave the tag6.7 D. This is why, our TEV was compared to commercial TEV (Promega) and as a negative control, thrombin. None of the proteases tested were able to cleave the tag. This is an indication of the fact that our target protein might have been not properly folded and MBP helped in its passenger solubilization. Also the MBP-CtAtg2(1-270) was tried in a purification experiment with the same results.

\subsection{The A. thaliana Atg18a-WRKY33 complex}

In A. thaliana, there are eight PROPPIN homologs [101]. One of these homologs, A. thaliana Atg18a (AtAtg18a), was recently found to interact with the transcription factor A. thaliana WRKY33 (AtWRKY33) in the nucleus [137]. This is the first time a PROPPIN was reported to have a function in the nucleus of a cell. This is why, we found this complex interesting to structurally characterize. This section presents the optimization 


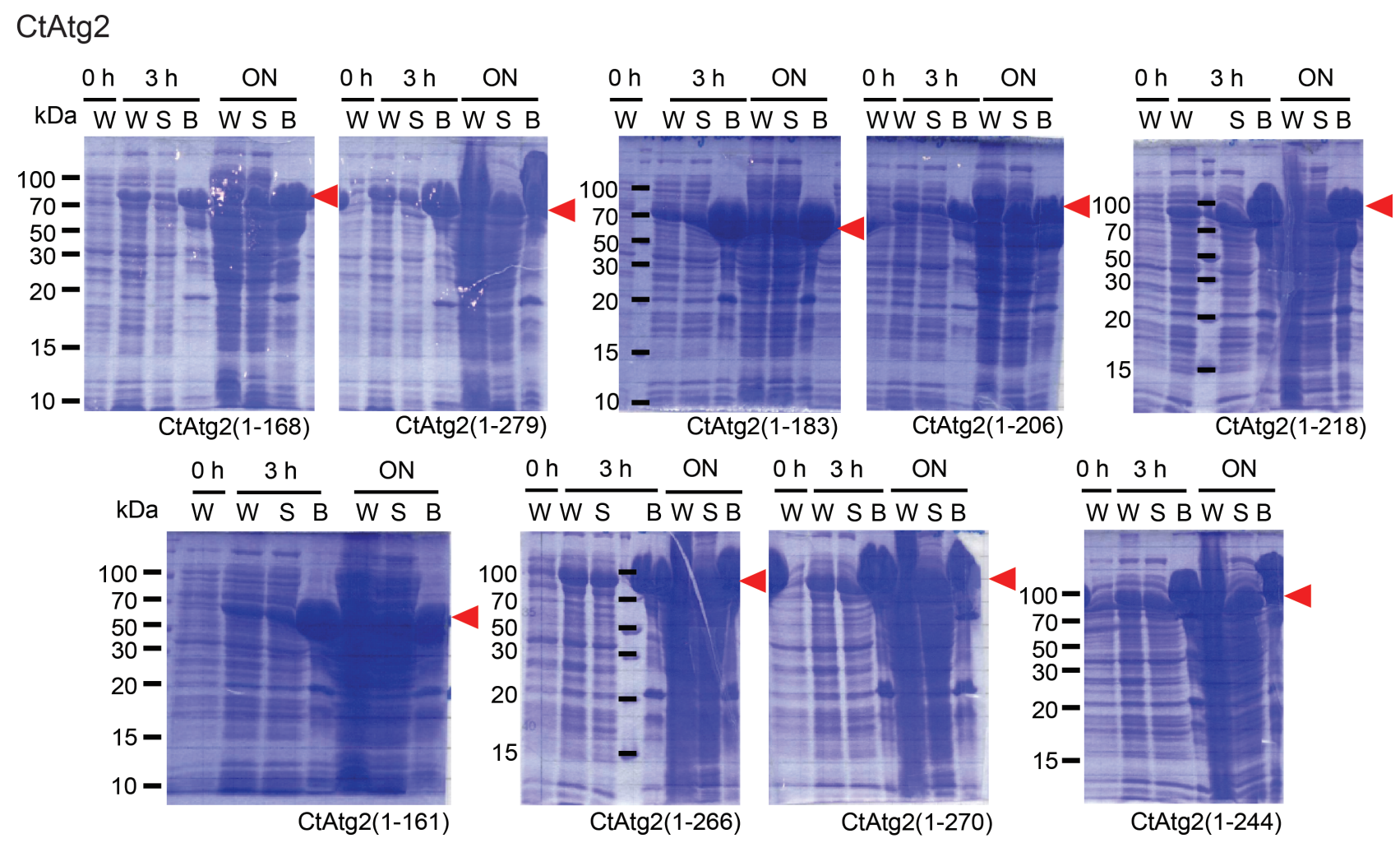

Figure 6.6: Expression optimization for CtAtg2 N-terminal fragments in E. coli.

SDS-PAGE gels showing the protein content of the expression samples harvested at different time points for different constructs. Expression media used was TB induced with $1 \mathrm{mg} / \mathrm{L}$ arabinose at $25^{\circ} \mathrm{C}$. The expression vector used was pBADM-41(+). Uninduced sample is represented as 0 hours. Samples were also harvested at 3 hours after induction and after overnight expression. W - whole cell lysate; $\mathrm{S}$ - soluble fraction; B - soluble fraction enriched on $\mathrm{Ni}^{2+}$-NTA beads; $\mathrm{ON}$ - overnight.

trials for the expression of AtAtg18a and AtWRKY33 in E. coli using the ACEMBL expression vector system [197].

The codon optimized for bacterial expression synthetic gene of AtAtg18a was cloned in the pAce1-N-His expression vector. This vector was chosen because of the possibility to recombine, using Cre recombinase, with the pDk vector, which can be used for cloning AtWRKy33. This would ease the expression and purification of the complex. An expression test was performed to determine the optimum expression condition for AtAtg18a in E. coli BL21(DE3). Several expression media and temperatures were tested: auto-inducing media (MD5052 and ZYM5052) and LB inducible by the addition of IPTG at $18^{\circ} \mathrm{C}$ and $37^{\circ} \mathrm{C}$. Samples were taken before induction with IPTG, for LB, or in the case of auto-inducing media, when $\mathrm{OD}_{600}$ was approximatively 0.5 . Further samples were taken at 3 and 5 hours after induction and after overnight expression.

Whole cell extract, soluble fractions and $\mathrm{Ni}^{2+}$-sepharose enriched soluble fractions were analyzed on SDSPAGE gels. In Figure 6.8, A, only the samples for MD5052 are shown. The expected size of AtAtg18a is 49 $\mathrm{kDa}$. On the SDS-PAGE gel, an enrichment of a $49 \mathrm{kDa}$ protein cannot be observed in the samples treated with $\mathrm{Ni}^{2+}$-sepharose beads. This is why, I have performed a Western blot detection of the $\mathrm{Ni}^{2+}$-sepharose enriched samples (Figure 6.8, B). A monoclonal anti-His tag antibody was used for detection. The protein presents as a double band, most probably due to the degradation of a part of the $\mathrm{C}$-terminal. However, for a short induction time at both $18^{\circ} \mathrm{C}$ and $37^{\circ} \mathrm{C}$ in MD5052 media proves to be the condition leading to a degradation product 
CtAtg2(1-279)

A

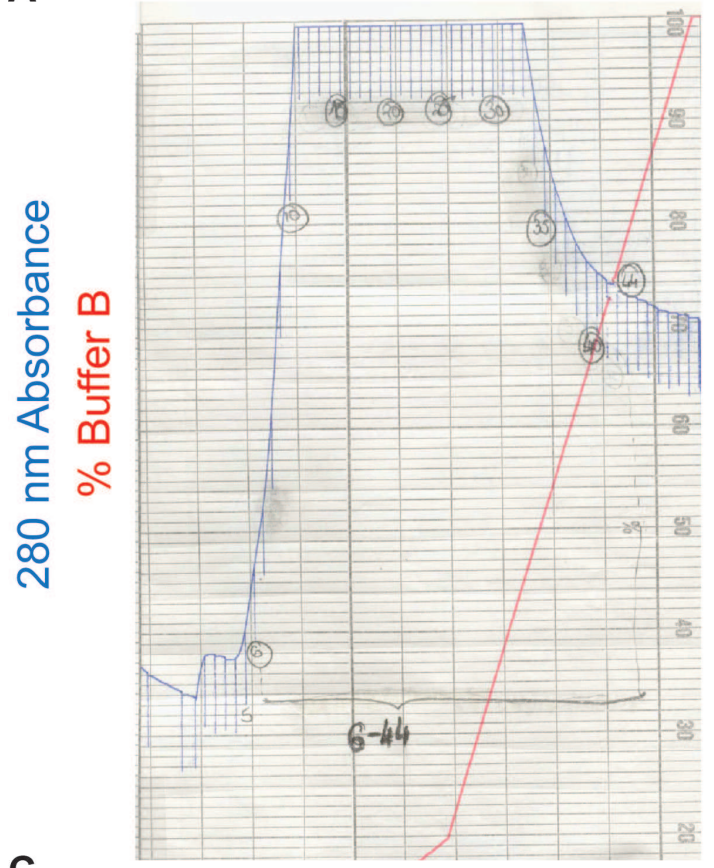

B

C
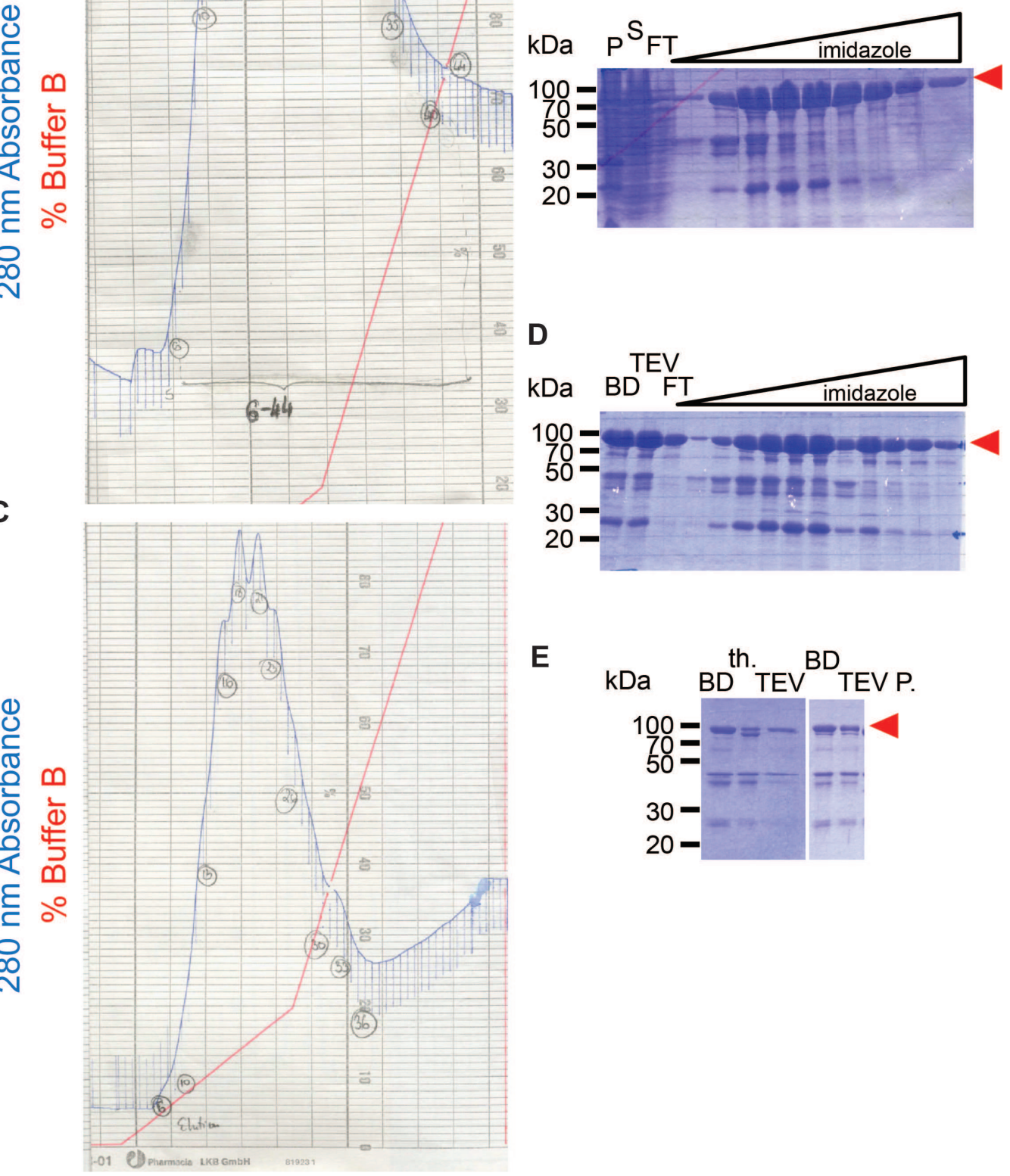

D

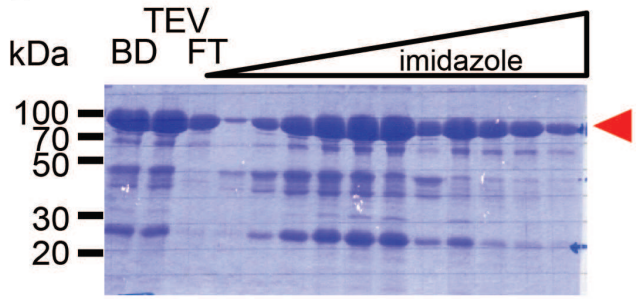

$\mathbf{E}$

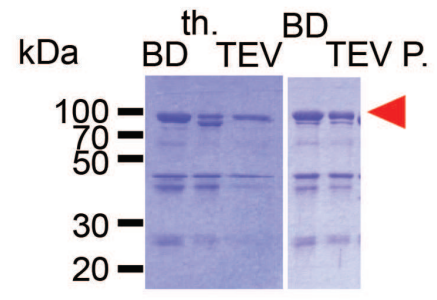

Figure 6.7: Purification of MBP-CtAtg2(1-279) from E. coli.

Chromatogram of MBP-CtAtg2(1-279) purified by (A) affinity chromatography using a HisTrap column followed by overnight MBPtag removal using TEV protease followed by another (C) affinity chromatography using a HisTrap column. The sample protein content can be followed on SDS-PAGE gels (C) for the first purification step and (D) for the second purification step. The elution fractions are shown in an increasing imidazole concentration. (E) Analysis of sample cleavage with TEV purified in our lab (TEV), commercial TEV (TEV P.) and thrombin. P - pellet; S - soluble fraction; FT - flow through ; BD - before dialysis/protease treatment; AD - after dialysis/protease treatment; th. - thrombin treatment. 
AtAtg18a

A

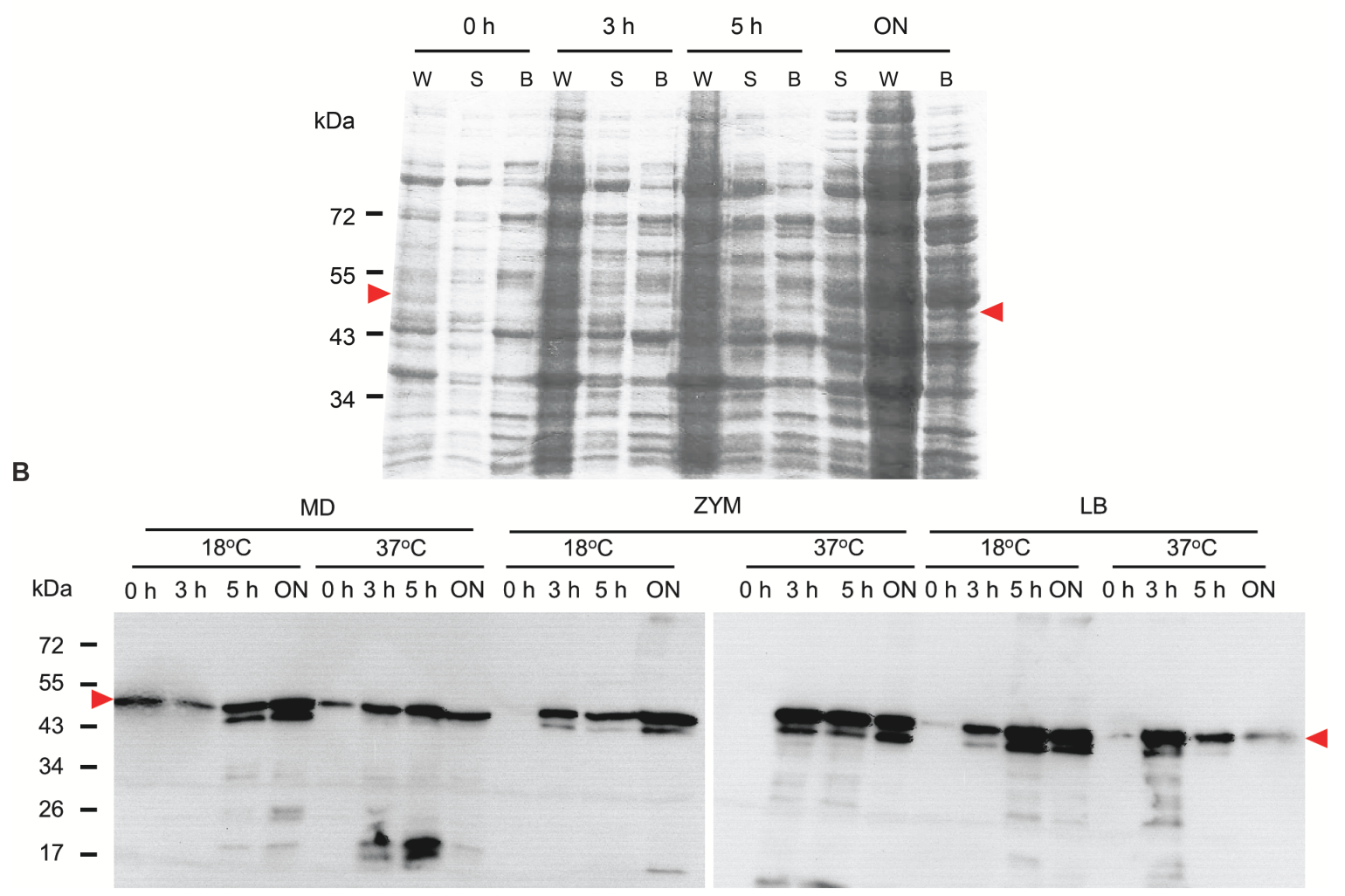

Figure 6.8: Expression optimization for AtAtg18a in E. coli.

Expression media tested were LB induced with $1 \mathrm{mM}$ IPTG and autoinducing media ZYM5052 (ZYM) and MD5052 (MD). Expression was analyzed for both $18^{\circ} \mathrm{C}$ and $37^{\circ} \mathrm{C}$. The expression vector used was pAce1-N-His. Uninduced samples are labeled as $0 \mathrm{~h}$. Samples were harvested at 3 and 5 hours after induction and after overnight expression. W - whole cell lysate; S - soluble fraction; B - soluble fraction enriched on $\mathrm{Ni}^{2+}$-sepharose beads; ON - overnight. Target protein is indicated by a red arrow. Samples collected during the optimization step were analyzed through SDS-PAGE, only for the MD5052 media (A), and Western blot detection with a monoclonal anti-His tag monoclonal antibody (B).

free sample. This is why, this expression condition was used for a purification experiment that yielded an unmeasurable amount of protein.

The synthetic gene used for AtWRKY33 was codon optimized for bacterial expression. The gene was cloned in the pAce1-N-StrepII expression vector. The same expression conditions as tested for AtAtg18a were as well used for AtWRKY331. Whole cell extract, soluble fractions and Strep-Tactin enriched soluble fractions were analyzed on SDS-PAGE gels. In Figure 6.9 A, only the samples for MD5052 are shown. The expected size of AtWRKY33 is $59.5 \mathrm{kDa}$. However, on the SDS-PAGE gel, in the affinity enriched samples, an enrichment of a protein of $59.5 \mathrm{kDa}$ cannot be observed. Similarly to AtAtg18a, I have performed a Western blot detection of the Strep-Tactin enriched samples using a monoclonal anti-Strep II tag antibody for detection (Figure 6.9, B). Two bads were detected through the Western blot, one which have an apparent size of $59.5 \mathrm{kDa}$ and a smaller one with an apparent molecular weight of $40 \mathrm{kDa}$. This is a possible degradation product of the full length protein. As in the case of AtAtg18a, the optimum expression condition seems to be a short induction time at $18^{\circ} \mathrm{C}$ in MD5052 media since the ration between the full-length protein and the degradation product is higher in this condition. This expression condition was used in a purification experiment that was unsuccessful. 


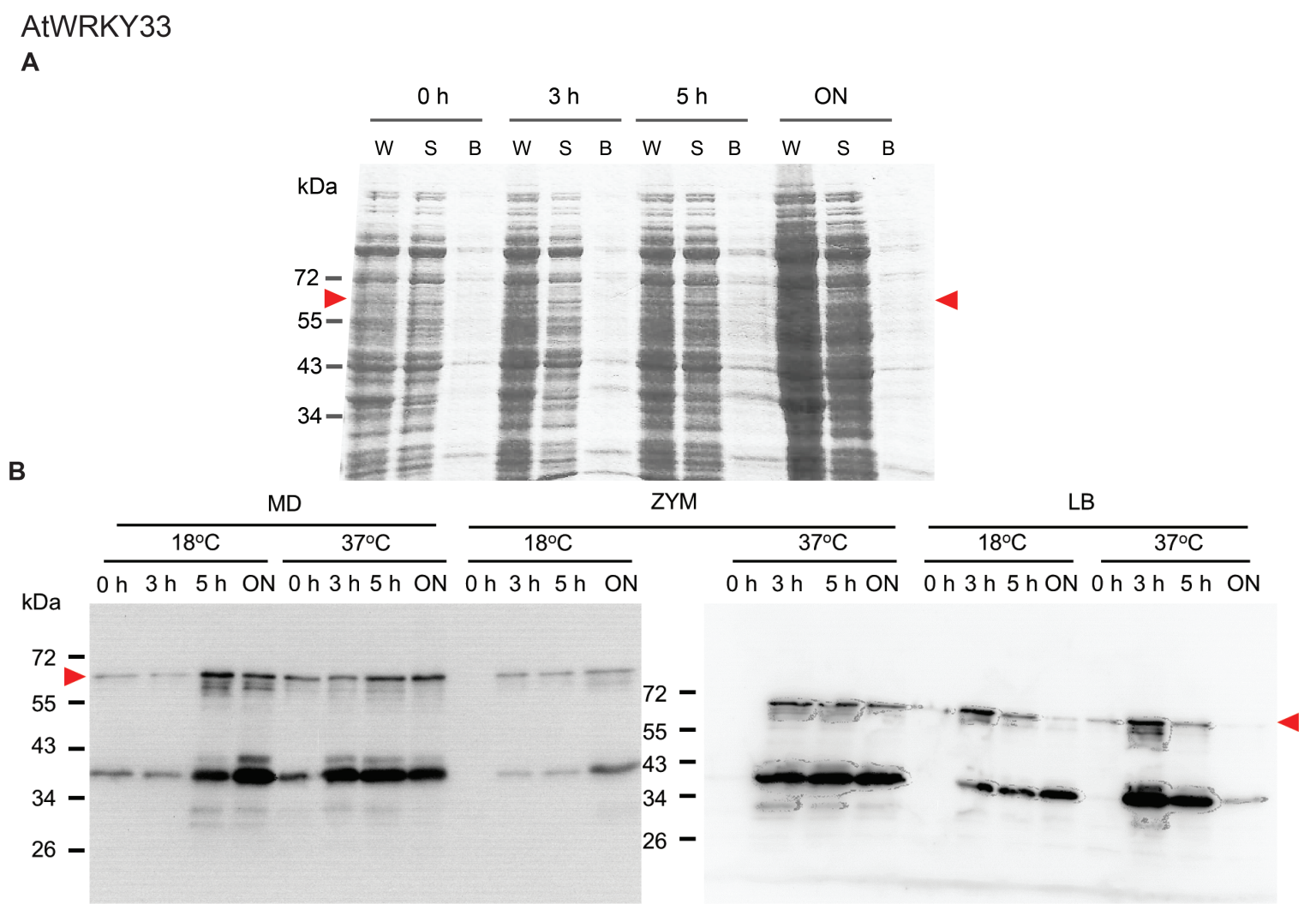

Figure 6.9: Expression optimization for AtWRKY33 in E. coli.

Expression media tested were LB induced with $1 \mathrm{mM}$ IPTG and autoinducing media ZYM5052 (ZYM) and MD5052 (MD). Expression was analyzed for both $18^{\circ} \mathrm{C}$ and $37^{\circ} \mathrm{C}$. The expression vector used was pAce1-N-StrepII. Uninduced samples are labeled as $0 \mathrm{~h}$. Samples were harvested at 3 and 5 hours after induction and after overnight expression. W - whole cell lysate; S - soluble fraction; B - soluble fraction enriched on Strep-Tactin beads; ON - overnight. Target protein is indicated by a red arrow. Samples collected during the optimization step were analyzed through SDS-PAGE, only for the MD5052 media (A), and Western blot detection with a monoclonal anti-StrepII tag monoclonal antibody (B). 


\section{Discussion}

\subsection{Computational studies of PROPPIN membrane binding}

The KlHsv2 structure was determined in the absence of PIs. Attempts to soak with PI-head groups were unsuccessful. In order to analyze whether crystal packing contacts might affect the accessibility for PI, MD binding simulations were performed. I showed that W277 does not shield binding site 2 and this was just a crystal packing induced conformation. Similarly, the structure of $K$. marxianus Hsv2 (KmHsv2) shows a similar flipped-away from binding site 2 conformation for W288 [2] (Figure 7.1). However, in both my MD simulations and in the crystal structure of KmHsv2, the first arginine in the FRRG motif remained in a conformation pointing away from the second arginine in the canonical PROPPIN binding motif.

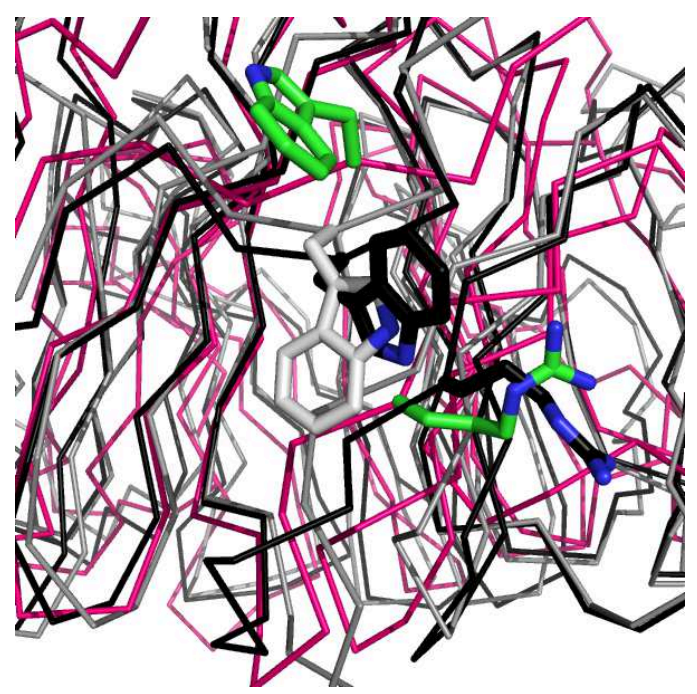

Figure 7.1: Comparison of conformation structures of the W277 and W288 in the determined structures of KIHsv2 (pdb accession code 4AV9, [1]) and KmHsv2 (pdb accession code 3VUA [2].

The two determined Hsv2 crystal structures are overlaid in a ribbon representation. KlHsv2 is represented in gray while KmHsv2 in black. KlHsv2 movement was simulated for $30 \mathrm{~ns}$ and the final conformation is depicted in pink ribbon. Final positions after the MD simulation of W277 and R219 are depicted in green sticks, while those determined from the crystal structure are depicted in gray (KlHsv2) and black (KmHsv2). KmHsv2 - K. marxianus Hsv2.

The preliminary MD simulations showed that, the two FRRG arginines will point towards opposite pockets. In addition, the sulfates defining the two binding pockets are $16.2 \AA$ apart. However, the distance between P1 and P3 in PI3P is $6.5 \AA$. These two facts put together, exclude the one binding site hypothesis. The fact that ScHsv2 has two binding sites was confirmed by ITC measurements with PI3P containing SUVs [1]. My ITC measurements further show that mutation in either binding site decreases PI3P affinity 15 to 30-fold explaining 
the necessity of two binding sites for membrane binding of PROPPINs. The two binding sites necessity is encountered by other PI binding domains which dimerize in order to solve this issue. This is the case for the PH domain of dynamin [71] and the FYVE domain of EEA1 [52].

The essential protein residues involved in ligand recognition are: H178, S198, D200, T202, R205 and R219 for binding site 1 and R220, K245, H249 and K283 for site 2. The docking for binding site 2 might not be as precise as for site 1 since the essential residue, K245 was disordered in the crystal structure. However, the findings of the computational experiments are in line with the in vitro binding experiments and in vivo localization studies [1]. In this study, the corresponding residues of site $1 \mathrm{~S} 198, \mathrm{~T} 202, \mathrm{R} 205, \mathrm{R} 219$ and site 2, R220, K245, H249 in ScHsv2 were shown to be important for membrane binding. However, H178 was not shown as essential in neither the biochemical experiments nor in the localization studies. Moreover, K283 was not tested in our lab, but was later proven essential by Baskaran et al. [30].

In my docked structures, the orientation of the inositol ring is similar to the ones of PI3P in the experimentally determined structure of FYVE domain [53]. In the structure mentioned, the ring is orientation such that $\mathrm{P} 3$ is towards the exterior of the binding pocket, while the hydroxyl on carbon C5 on the ring is towards the inside of the binding site. This orientation, which is found over several PI3P binding proteins, might not be coincidental; the inositol ring orientation with respect to the surface of the lipid membrane adopts restricted range of conformations [204]. Hence, it might be the case that nature has evolved proteins to recognize this constrained ring conformation.

Furthermore, $\mathrm{PI}(3,5) \mathrm{P}_{2}$ docking was successful only when $\mathrm{H} 178$ and $\mathrm{H} 249$ were protonated. One can speculate that the recognition of $\mathrm{PI}(3,5) \mathrm{P}_{2}$ is done at low $\mathrm{pH}$ through a histidine switch as observed for the $\mathrm{PH}$ domain of GRP1 protein [65] and by the FYVE domain of EEA1 [66]. If indeed a histidine switch is involved in PROPPIN membrane binding, its effect should be stronger in the case of ScHsv2 which has a total of three histidines involved in PI binding. In ScHsv2 binding pocket 2, H345 binds P3, while in KlHsv2, this is done by K283. In order to experimentally probe this hypothesis, Dr. Ricarda Busse has performed ITC experiments by titrating KlHsv2 and ScHsv2 into PI containing SUVs at different pH [192]. However, the experiments were unsuccessful most probably due to the fact that SUVs and the proteins are not stable over a large range of $\mathrm{pH}$ values.

The mutagenesis experiments mentioned above, suggested that some KlHsv2 residues bind tighter to PIs than others. In order to study this hypothesis on an atomistic level, I have performed MD simulations with both $\mathrm{PI} 3 \mathrm{P}$ and $\mathrm{PI}(3,5) \mathrm{P}_{2}$ in both binding sites. $\mathrm{MD}$ simulations have shown that the weakest protein-ligand interactions are the salt-bridges with $\mathrm{P} 1$. The same behavior was previously observed in similar MD simulations of the PH domain of PLC $\delta 1$ and the FYVE domain of EEA1 [205, 206].

Interestingly, in the $\mathrm{PI}(3,5) \mathrm{P}_{2}$ simulations, the ligands drifted away from the initial configuration, equivalent with the phosphoinositides being pulled out of the eventual lipid bilayer. This might be an artifact due to the fact that we simulated free PIs in solution rather than membrane constrained phosphoinositides. As previously shown in similar studies [205, 206], in simulations with membrane bound PIs, the protein-ligand interactions are stronger.

The MD simulations and docking studies have helped in proposing a model for membrane recognition by KlHsv2 (Figure 3.9). In this model, there are two PI binding sites and two hydrophobic loops penetrating into the membrane that together contribute synergistically to strong membrane docking of KlHvs2. This mode of 
membrane binding was observed for other PI binding domains, e.g., the PX domain of yeast Vam7p [75] and the FYVE domain of EEA1 [76].

In order to deeper look into the model mentioned above, CG-MD simulations were performed. They showed that KlHsv2 loop 6CD can be found either in solution or in membrane as a lipid bilayer formed around this loop six out of fifteen times. Moreover, in the case of the atomistic MD simulations, loop 6CD goes through several cycles of folding and unfolding. These findings can be explained by the fact that a loop that is inserted in the membrane has to have to ability readily change conformation and refold while being stabilized by the hydrophobic contacts.

In vivo, PROPPINs are found both free in the cytosol and recruited to PAS upon autophagy induction. This is why, it makes sense that loop 6CD should have this dual character. Moreover, in P. pastoris the phosphorylation of loop 6CD leads leads to the regulation of Atg18 membrane binding [106]. Moreover, in our lab, mutagenesis experiments involving shortening the loop and replacing it by a GS linker proved this loop essential in membrane binding (Figure 8.1 D).

Sequence alignment of Hsv2 homologs across species revealed that loop 6CD is not conserved in sequence, nor in length [192]. However, there is a conservation in the chemical properties of the residues involved. Aromatic residues W267, Y272 and F273 together with a basic patch found in the middle of K1Hsv2 loop 6CD (K260, R261 and H262) were shown to be important for membrane binding using liposome co-flotation assay (Figure 8.1 D). The aromatic residues were previously studied, but a triple mutant was investigated and the contribution of each residue was not assessed [30].

In order to dissect which residues in loop 6CD are important for membrane binding, atomistic MD simulations were performed for the protein-membrane system. It was concluded that once bound to the membrane, KIHsv2 oscillates around the loop making more membrane contacts with residues in the vicinity of binding site 1. Residues R262 and H262 make cloe to 1 membrane contact/ps, while W267 and Y272 make close to 0.7 contacts/ps. The K260 and F273 are in little contact with the membrane. This electrostatics-hydrophobic interplay involved in the membrane binding of a PI domain can be also found in the C2A domain of synaptotagmin 1 and 7, [74]. in the PX domain of yeast Vam7p [75] and the FYVE domain of EEA1 [76].

Non-specific charge interactions seem to be important in PI domain membrane recruitment.Theoretical studies showed how the electrical field created by the charges of a membrane, can orient PH domains in the right direction and bring them close to the membrane [73]. In order to experimentally validate, this hypothesis for KlHsv2 membrane binding, a FRET based assay using dansyl-labeled LUVs was performed with PI3P and PS containing liposomes (Figure 8.1, A-C). It was shown that while PI3P binding is tighter, KlHsv2 binds faster to PS vesicles (Figure 8.1, E). Hence, first the electostatic based interactions recruit PROPPINs to the membrane where they further bing PIs.

All in all, the computational study, together with experimental evidence allowed us to propose a model for membrane recruitment and binding (Figure 7.2). The negatively charged membrane orients the approaching PROPPIN in such a way that loop 6CD is in the proximity of the membrane. The positive patch in the middle of loop 6CD helps the PROPPIN reach further towards the membrane and attach to it. Once loop 6CD inserts, the PROPPIN diffuses along the membrane, oscillating around loop 6CD, with a preference towards the basic binding sites, especially binding site 1 . The diffusion and oscillation are stopped when the PROPPIN encounters PIs and specifically binds to them, stabilizing its membrane interaction. 

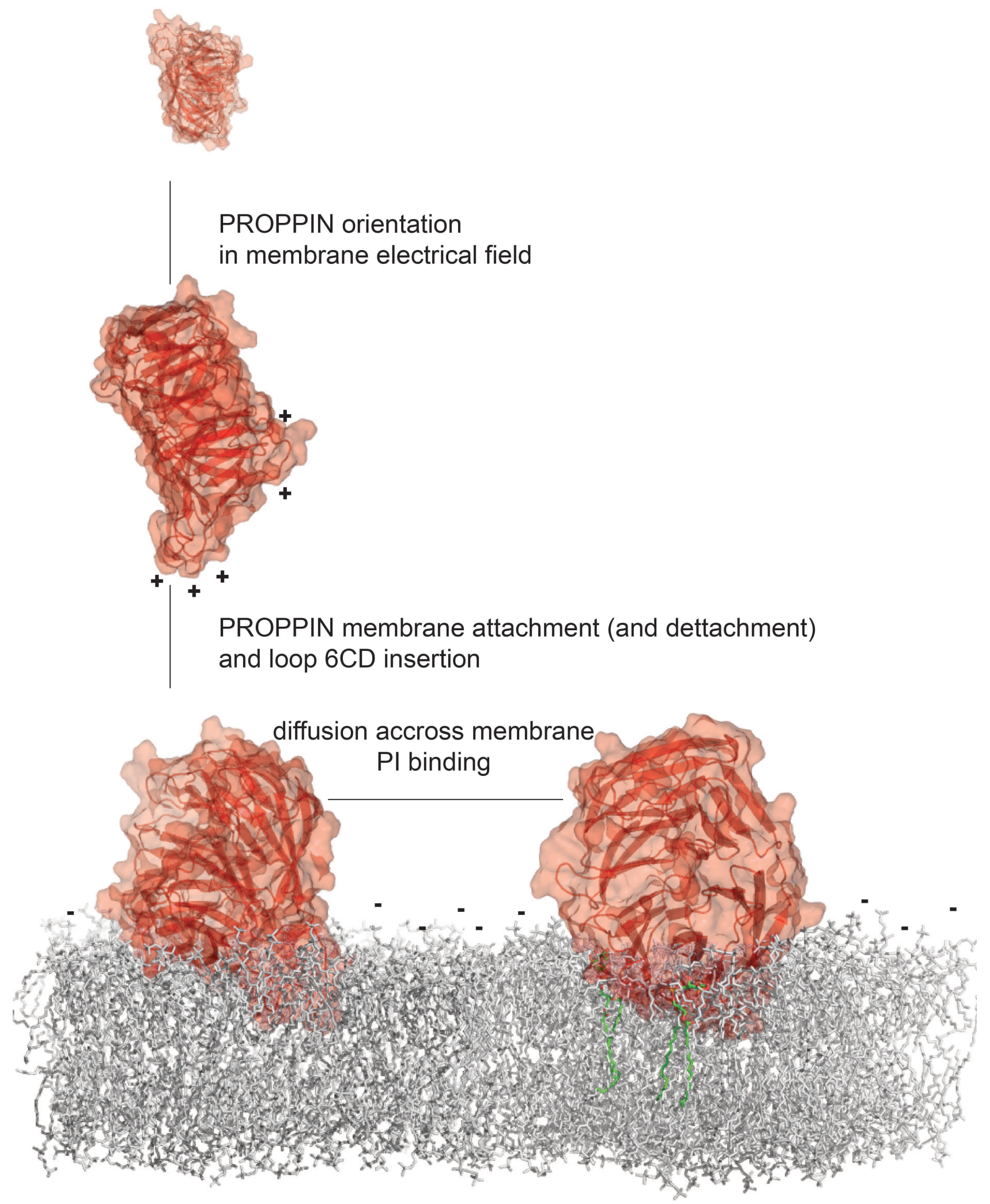

Figure 7.2: Proposed model for PROPPIN membrane recruitment and binding. The negatively charged membrane creates an electrical field that orients the approaching PROPPIN in such a way that loop 6CD is in the proximity of the membrane. The positive patch in the middle of loop 6CD helps the PROPPIN reach further towards the membrane and attach to it. This attachment is weak and the PROPPIN detaches until loop 6CD inserts using its aromatic residues. Once loop 6CD inserts, the PROPPIN diffuses along the membrane, oscillating around loop $6 \mathrm{CD}$, with a preference towards the basic binding sites, especially binding site 1 . The diffusion and oscillation are stopped when the PROPPIN encounters PIs and specifically binds to them, stabilizing its membrane interaction. 
The proposed PROPPIN binding mode requires further validation to prove that indeed this is the correct orientation with respect to the membrane. Other questions opened by this study are the ones related to the differences observed for binding site 2 across paralogs; while Hsv2 have a basic residue binding P5, Atg18s and Atg21s have a polar one. Moreover, it would be interesting to study each binding site independently, to investigate whether there is a reason binding site 1 is more conserved and which binding site binds first a PI. Furthermore, the possibility of a $\mathrm{pH}$ switch is worth exploring further.

In order to experimentally address any of these questions, PROPPINs must be expressed and purified, as done in the next section.

\subsection{Biochemical characterization of phosphoinositides binding specificities of different PROPPIN family members}

In this part, I expressed and purified the different PROPPIN family members, ScAtg18, ScAtg21 and CtAtg18. Their bacterial expression was unsuccessful despite trying different expression conditions such as expression strains, growth media and incubation temperatures.

Since none of these PROPPINs were successfully expressed, I turned to the already established protocols from Dr. Ricarda Busse for the expression and purification of PaAtg18, KlAtg21 and ScHsv2. In the case of PaAtg18 and KlAtg21, the expression and purification was slightly modified towards improved yields. The purified proteins were folded, as tested by CD spectra and melting curves.

Next, the thermodynamical binding parameters of PROPPINs to SUVs containing either PI3P or PI(3,5) $\mathrm{P}_{2}$ were measured. With the exception of KlAtg21 binding to $\mathrm{PI}(3,5) \mathrm{P}_{2}$, all PROPPIN PI3P or PI $(3,5) \mathrm{P}_{2}$ interactions had submicromolar binding affinities. In general, the binding to $\mathrm{PI}(3,5) \mathrm{P}_{2}$ is stronger than to $\mathrm{PI} 3 \mathrm{P}$, with the exception of K1Atg21 which binds both PIs similarly tight. When compared to the other reported literature values (Table 7.1), one generally observes agreement.

Table 7.1: Summary of reported PROPPIN binding affinities.

\begin{tabular}{ccccc}
\hline PROPPIN & Ligand & $\mathbf{K}_{d}(\mu \mathbf{M})$ & experimental setup & reference \\
\hline ScAtg18 & $\mathrm{PI}(3,5) \mathrm{P}_{2}$ & 0.5 & surface plasmon resonance & {$[104]$} \\
ScAtg21 & $\mathrm{PI}(3,5) \mathrm{P}_{2}$ & 0.5 & surface plasmon resonance & {$[104]$} \\
ScHsv2 & $\mathrm{PI}(3,5) \mathrm{P}_{2}$ & 0.5 & surface plasmon resonance & {$[104]$} \\
ScHsv2 & $\mathrm{PI} 3 \mathrm{P}$ & 0.67 & ITC & {$[1]$} \\
GST-ScHsv2 & $\mathrm{PI3P}$ & 1.3 & reflectometric interference spectroscopy & {$[1]$} \\
KlHsv2 & $\mathrm{PI3P}$ & submicromolar & FRET based assay with LUVs & {$[30]$} \\
KlHsv2 & $\mathrm{PI} 3 \mathrm{P}$ & 9.61 & FRET based assay with LUVs & our group \\
KlHsv2 & $\mathrm{PI3P}$ & 0.5 & ITC with SUVs & {$[192]$} \\
KlHsv2 & $\mathrm{PI}(3,5) \mathrm{P}_{2}$ & 0.18 & ITC with SUVs & {$[192]$} \\
\hline
\end{tabular}

The small differences in binding affinities determined could be due to experimental setup differences, directly measured parameters, membrane content and protein sample quality. In terms of membrane content differences, in the case of GST-ScHsv2 binding to PI3P measured through reflectometric interference spectroscopy, membranes containing 3\% PI3P were used [1]. Similarly, in the Baskaran et al. study, 5\%PI was used in addition to 55-60\% PS. In our laboratory, it was shown that negatively charged SUVs non-specifically 
bound ScHsv2 [155]. Moreover, in the reflectometric interference spectroscopy experiments [1], the curvature of the membrane surface is much larger than in my case. Finally, using a GST-fusion construct, leads to an apparent higher binding affinity due to its dimerization properties. This was previously shown in the case of ScAtg18 which had a three times higher binding affinity in the fusion version when compared to wild type protein [104]. In terms of protein sample quality importance, when comparing the binding affinity of ScHsv2 to PI3P detemined by myself and the one our group previously reported [1], I got $0.40 \mu \mathrm{M}$, while the reported value was $0.67 \mu \mathrm{M}$. As shown in Figure 4.8, I had difficulties removing the fusion protein and the free GST from the final ScHsv2 protein sample, which was not a problem for Dr. Ricarda Busse, who developed the original protocol [192].

The only large deviation from the submmicromolar binding affinity, was the FRET based stopped-flow measurements performed in our group on KlHsv2. This experiment led to a dissociation constant for KlHsv2PI3P system of $9.61 \mu \mathrm{M}$. One explanation could be the fact that in my experiments, I have used SUVs, while in the FRET experiments, LUVs were used. Baskaran et al. also used LUVs in a similar experiment and obtained submicromolar dissociation constant, however, their binding strength was increased due to highly negatively charged LUVs used in the experiments. When discussing membrane curvature, it was previously showed in our laboratory, through liposomes co-flotation assays that KlHsv2 membrane binding is dependent on vesicle curvature, i.e., KlHsv2 bound weaker to PI containing LUVs than to SUVs [155]. The importance of membrane curvature in binding PI containing membranes is a recruitment mechanism for other PI binding domains such as sorting nexin-1 [78, 77]. The importance of membrane curvature for PROPPIN membrane interaction would be interestingly to investigate since, in cells, Atg2-Atg18 complex is localized at the edge of the isolation membranes in close proximity to the ER exit sites [138]. This implies that Atg2-Atg18 complex prefers the localization to high curvatures areas of the PAS. Moreover, once the autophagosome is grown and enclosed, i.e., when the membrane curvature increased, the complex detaches from the autophagosomal membrane [142].

In terms of binding stoichiometries, I determined values in between 1.4 and 3.3 PI molecules binding per protein molecule. However, the binding stoichiometry of PI3P to PaAtg18 could not be determined because I could not do a proper fitting on the titration curves obtained. This was due to the fact that the measured signal was weak.

The differences from the expected value of two might be explained by the fact that, as shown in the purification analysis on SDS-PAGE gels in Figures 4.6, 4.7 and 4.8, the protein samples used are not homogeneous. Contaminants contribute to the protein concentration determination, a value essential for proper titration curve fitting. Furthermore, the exact determination of the PI concentration is crucial. The PI concentration is determined from total phosphate concentration. Considering that several phosphate containing lipids are mixed, the errors cannot be neglected. Moreover, as I have shown the loop 6CD membrane insertion has an important role in PROPPIN membrane binding. This insertion would contribute to the heat released from the PROPPIN membrane binding measured by ITC. However, the model used for fitting is a one site model in which only one type of binding, with single or multiple similar pockets. is assumed. However, this is not the case in my system, since the two PROPPIN binding pockets are not identical and the loop 6CD insertion cannot be overlooked. Furthermore, in the cases of $\mathrm{ScHsv} 2$ and $\mathrm{PaAtg} 18 \mathrm{PI}(3,5) \mathrm{P}_{2}$ titration curves, the two binding sites fitting model was more appropriate. In spite of this, the one binding site fitting model was chosen due to a lack of consistency from one experiment to another in the determined thermodynamical parameters for the two sites binding fitting model. 
It was not possible to determine the stoichiometries for the single site binding mutants of ScHsv2. This results is in line with what was previously reported for the same single binding sites mutants using reflectometric interference spectroscopy [1].

Overall, for all PROPPINs, the binding is enthalpically driven. Enthalpic binding is a result of specific contacts, i.e., salt bridges and hydrogen bonds, while entropic driven binding is due to nonspecific hydrophobic interactions.

For PaAtg18 and KlAtg21, the entropic an enthalpic contributions to the binding are larger in the case of $\mathrm{PI}(3,5) \mathrm{P}_{2}$ than in the case of PI3P. However, for ScHsv2, the reverse is true. This pattern was also observed for KlHsv2 (Figure 8.2), as measured by Dr. Ricarda Busse [192]. This observation is interesting, since the Hsv2s have in their binding pocket a lysine or histidine residue binding P5, while the Atg18s and Atg21s have a polar residue in the same position.

Taken together, PI was measured for each PROPPIN and the thermodynamic binding parameters were determined. The PROPPIN PI binding is enthalpically driven and in general, $\mathrm{PI}(3,5) \mathrm{P}_{2}$ binds stronger. A difference was noticed for the contribution of entropy and enthalpy to the binding energy for PI3P vs. PI(3,5) $\mathrm{P}_{2}$ for Hsv2 compared to Atg18s and Atg21s. However, during this investigation, some questions appeared. It would be interesting to study the importance of membrane curvature for the PROPPIN binding to membranes. Moreover, it would seem important to be able to dissect the loop 6CD membrane insertion from the process of PI recognition and binding. Finally, the origin of differences in thermodynamic contributions in the binding of PI3P vs. PI(3,5) $\mathrm{P}_{2}$ among different PROPPINs could be further investigated.

In order to answer some of these questions the structures of Atg18 and Atg21 would be needed. This is why, I determine the structure of PaAtg18.

\subsection{Structural and biochemical characterization of P. angusta Atg18}

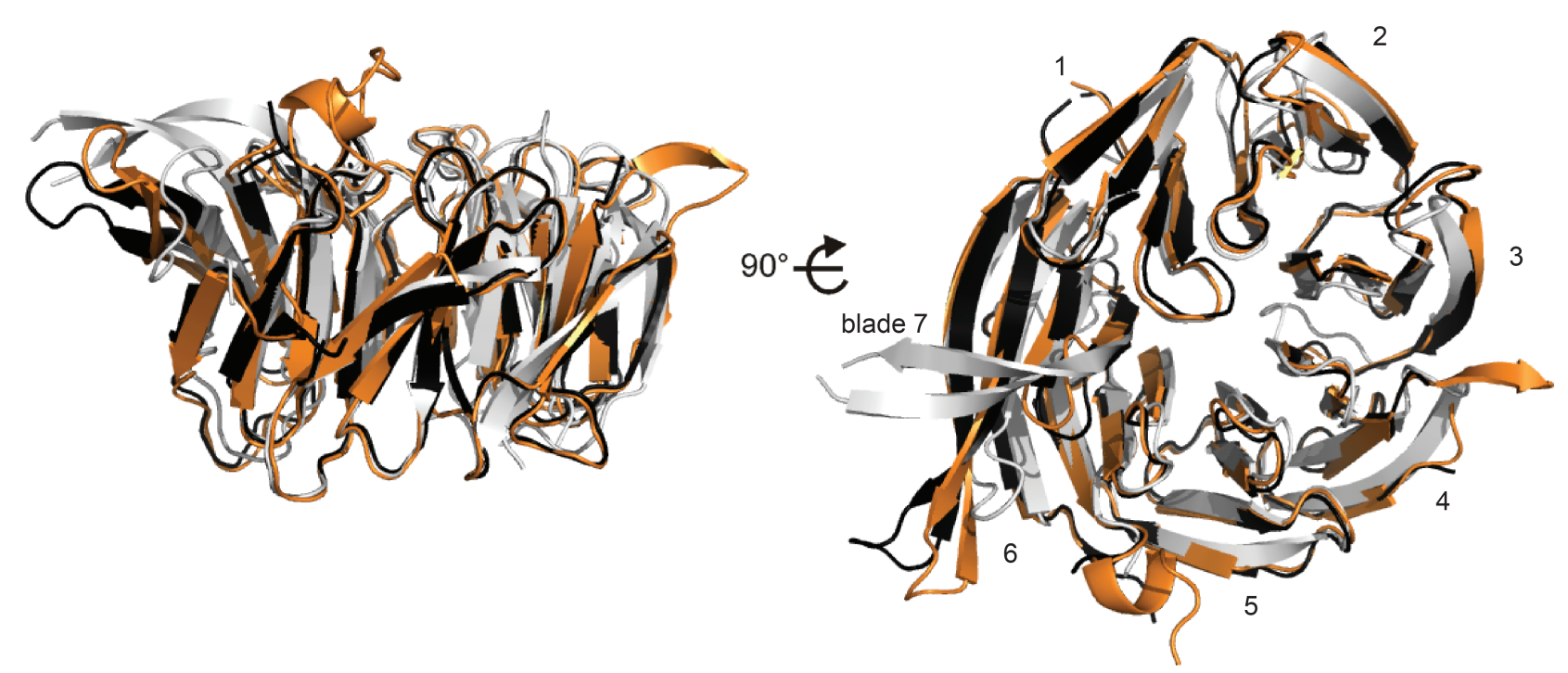

Figure 7.3: Comparison between PROPPIN structures. The structure of PaAtg18 (gray) is overlaid with both KlHsv2 (orange) pdb accession code 4AV9, and Kluyveromyces marxianus Hsv2 (black) - pdb accession code 3VUA [2] 
I determined the PaAtg18 PROPPIN structure at $1.8 \AA$ resolution. Like the previously determined KlHsv2 and KmHsv2 structures [1, 30, 2], PaAtg18 is a seven bladed $\beta$-propeller with a non-velcro closure topologies. Overlay of PaAtg18 with the other two determined PROPPIN structures (Figure 7.3) shows that their structures are very similar, except for strands 7A and 7B, which are longer in PaAtg18. These two strands protrude outside the $\beta$-propeller. The RMSD value for the PaAtg18 overlay with KlHsv2 is $1.56 \AA$ and $1.52 \AA$ for KmHsv2.

Notably, PaAtg18 (525 residues) is much longer than KlHsv2 (325 residues) and KmHsv2 (355 residues). Since the strands of the propellers have similar lengths, the almost 200 a.a. difference is due to the variations in length of the loops connecting these strands. Longer loops $(4 \mathrm{AB}, 6 \mathrm{CD}$ and $7 \mathrm{AB})$ did not have an electron density in the PaAtg18 structure. Loop 6CD was disordered in the case of KlHsv2. This loop is important in unspecific membrane binding. In PaAtg18 loop 6CD is approximatively 150 a.a. in length. This loop contributed to membrane binding as shown for KlHsv2 and P. pastoris Atg18 [106]. Moreover, the computational membrane binding model I proposed for PaAtg18 shows that loop 7AB would possibly insert in the membrane, as it is the case for KlHsv2.

Likely due to the longer loops in PaAtg18, I was unable to obtain well diffracting crystals. In situ proteolysis was essential for the determination of a good resolution structure. Unfortunately, due to high PEG 8000 concentration in the crystallization condition. I wanted to analyze the crystallized fragment by in situ proteolysis by SDS-PAGE. I was unable to obtain clear bands on an SDS-PAGE gel of the dissolved crystal. However, while analyzing the digestion pattern of several proteases, Dr. Ricarda Busse [192] concluded that Proteinase K would digest PaAtg18 in proteins of apparent approximative sizes of $20 \mathrm{kDa}, 15 \mathrm{kDa}, 12 \mathrm{kDa}$ and smaller than $10 \mathrm{kDa}$. The four fragments present in the crystal structure, namely 33-179, 201-297, 441-467 and 485-524 would have the molecular weights of approximatively $20 \mathrm{kDa}, 12 \mathrm{kDa}, 3 \mathrm{kDa}$ and $6 \mathrm{kDa}$. This is in good agreement with the observations done by Dr. Ricarda Busse. Hence, the missing loops might be the result of the proteolytic digest by Proteinase K, rather than flexibility in the crystal. Moreover, Dove et al. have performed proteolytic digestion analysis on ScHsv2 with trypsin resulting in two fragments, 1-377 and 378-500 [104]. Based on the multiple sequence alignment I have done for the close yeast homologs of PaAtg18 (Figure 5.13), residue 377 in ScAtg18 corresponds to the start of loop 6CD. Therefore, loop 6CD is susceptible to protease cleavage due to surface exposure in more than one species.

In terms of surface conservation, the two PI binding pockets defined by the FRRG motif are highly conserved in both PaAtg18 and KlHsv2. PaAtg18 has two additional conserved surface patches on the rims of blades 1 and 2 and blade 4. Mutagenesis studies have shown that residues in blades 1 and 2, shown in Figure 7.4 are important in Atg2-Atg18 complex formation [139, 2]. For the conserved region in blade 4 there is no functional information available. Moreover, loop $4 \mathrm{AB}$ conserves highly conserved regions and is not conserved among yeast Atg18 homologs. Since it was digested by Proteinase K, one can speculate that this loop is surface exposed and highly flexible. It might be the case that it has functional importance, a hypothesis strengthened by its proximity to a conserved surface region. The presence of these two conserved regions stresses the fact that as a WD40 domain, PaAtg18 is a hub of protein-protein interaction. Thus, the structure of PaAtg18 sets the basis for further studies involving Atg18 and its interaction partners.

However, when discussing the surface conservation, one has to consider that I have done this analysis based on close homologs to PaAtg18. If one would like to investigate highly conserved interactions, such as the Atg2-Atg18 might be, they have to perform more in depth bioinformatics analysis with considerable more sequences. 


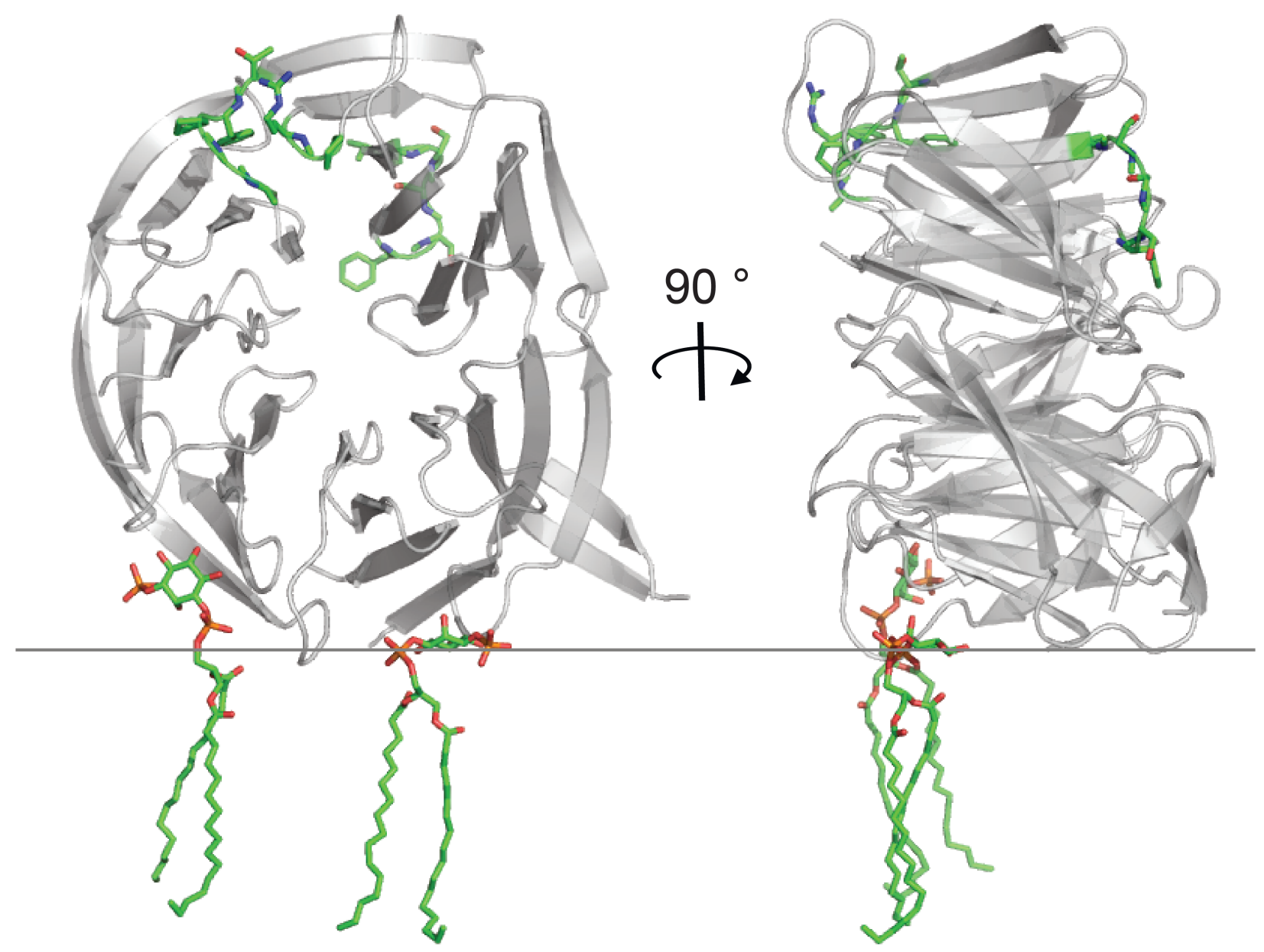

Figure 7.4: Atg2 binding sites on PaAtg18. In the PaAtg18 membrane binding model it is revealed that Atg2 binding sites, shown in green sticks [2, 139] are on the opposite site of the PI binding sites. The membrane is depicted through a gray line for simplicity.

In terms of PI binding, the two arginines of the FRRG motif in PaAtg18 point towards opposite directions as it it is the case for the structure of KlHsv2. In fact, when overlaying PaAtg18 and KlHsv2 structures, the two arginines perfectly overlap (Figure 5.12). This overlap is found for all the essential residues in PI binding site 1 , and most of the important residues of PI binding site 2, with the exception of T288 which is correspondent to $\mathrm{K} 283$.

I have determined two PaAtg18 structures with different crystal bound ligands found in the vicinity of the FRRG motif. In the $2.3 \AA$ crystal structure, phosphates were found bound to the same positions as the sulfates in KlHsv2. Sulfates and phosphates bound to the PI binding sites in PI binding domains indicate the position of PI phosphates. As I showed through computational docking studies, in the case of the KlHsv2 and PaAtg18, sulfate/phosphate 1 corresponds to P5, while sulfate/phosphate 2 represents the position of P1. Thermofluor analysis showed that phosphate buffer stabilizes PaAtg18. Interestingly, this is the case also for ScAtg18. In the $1.8 \AA$ crystal structure, a phosphate was bound in the same position as before in pocket 2, but in PI binding site 1 a citrate originating from the crystallization buffer was bound to this structure. It appears that citrate is stabilizes PaAtg18 as it was also found in the condition for the crystallization of the full length protein which contained ammonium citrate dibasic. 
Finally, in order to experimentally test the PROPPIN membrane binding model, together with Dr. Ángel Perez Lara, I have setup a fluorescence based reporter system in which the membrane proximity of different sites on the $\beta$-propeller is probed. The necessary PaAtg 18 mutants were purified and need to be measured.

In this part I determined the structure of PaAtg18 which raised questions related to its binding partners, Atg2 in the surface conserved region in blades 1 and 2, and unknown binding partner(s) for blade 4 . The structural studies of the protein complexes involving Atg18 is the next natural step. This is why, next I have tried to express and purify ScAtg2 and CtAtg2 and the AtAtg18a-AtWRKY33 complex.

\subsection{Expression and purification of PROPPIN binding partners}

In the study of the Atg2-Atg18 complex there is a standstill when it comes to the way the complex is recruited to the membrane. On one hand, Ohsumi proposes that the complex forms in the cytosol and are together recruited to the PAS. On the other hand, Reggiori proposes that Atg2 first binds to the PAS and then recruits Atg18 (Figure 1.4).

Structural studies of the Atg2-Atg18 complex would help to understand how this complex is recruited. The expression and purification of Atg2 is essential for structural studies. However, up to now, no soluble Atg2 domain or full length protein were obtained from yeast or bacterial expression. Beside the full length expression in yeast, Atg2 N-terminal domains from both S. cerevisiae and C. thermophilum were tested with no success. Since it was shown that human Atg2A interacts with DnaJ chaperones in vivo, one could try the co-expression of Atg2 in insect cells with chaperones or its natural interaction partner, Atg18.

Another target complex for this study was the A. thaliana Atg18a-WRKY33 complex. The fact that an autophagy protein interacts with a transcription regulator is intriguing. Considering that both PI3P and PI5P are present in the plant nucleus [153] and that PROPPINs can bind PI5P [30, 207], makes this PROPPIN very interesting. Moreover, there is no PI5P bound structure available. This is why, the structure of the plant Atg18aWRKY33 complex might give insights not only in the biological process of plant immune response, but also into the biochemistry of the PROPPIN PI binding and PI5P binding in general.

Unfortunately, none of these questions could be answered since the expression conditions tested were unfavorable for both proteins. In the future, one could try co-expression in insect cells.

\subsection{Outlook}

In this study I determined the structure of PaAtg18. A next step would be to structurally study its interaction partners. I tried to express Atg2 domains and full length protein in budding yeast and bacteria, but I was unsuccessful. One strategy would be the Atg2-Atg18 complex expression in the presence of chaperones in insect cells. This could also be the answer for the failed expression of AtAtg18a-AtWRKY33 complex.

Moreover, a PROPPIN binding orientation is proposed based on computational methods. This model has to be experimentally tested. In our laboratory, we have established a fluorescence based method to do so and the PaAtg18 mutants were expressed and purified. Further experiments need to be done for a complete view on the PROPPIN membrane orientation.

ITC measurements have revealed differences in thermodynamic contributions in the binding of PI3P vs. $\mathrm{PI}(3,5) \mathrm{P}_{2}$ among different PROPPINs. This fact might be intertwined with the observation that there are dif- 
ferences in the residues involved in PI binding site 2 across paralogs, i.e., while Hsv2 have a basic residue binding P5, Atg18s and Atg21s have a polar one. This can be studied by mutating the basic residue into a polar one in Hsv2 and the polar one into a basic residue in Atg18 and Atg21 and measuring thermodynamic binding parameters using ITC.

Moreover, it would seem interesting to be able to dissect the loop 6CD membrane insertion from PI binding. Preliminary experiments using FRET based kinetic stopped flow have showed that two regions can be distinguished in the fitting of binding kinetics. This might allow the measurement of the contribution of each binding component to PROPPIN membrane binding.

Further questions raised by this research are the importance of membrane curvature for PROPPIN membrane binding and whether the PI binding. 


\section{Appendix}
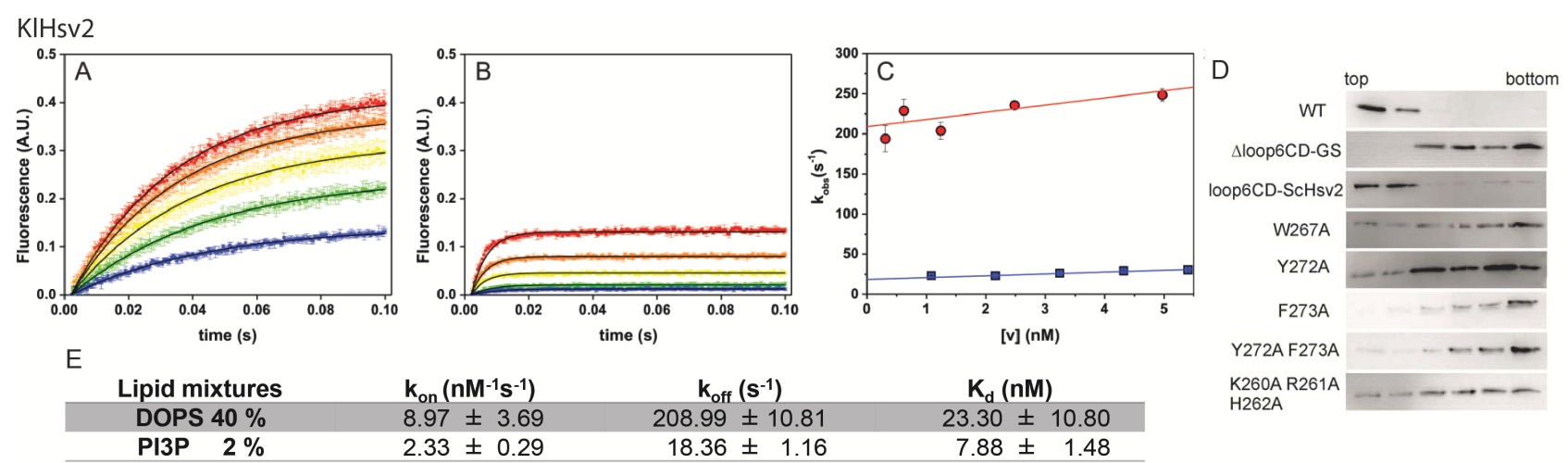

$\begin{array}{cc}\mathbf{k}_{\text {on }}\left(\mathbf{n M}^{-1} \mathbf{s}^{-1}\right) & \mathbf{k}_{\text {off }}\left(\mathbf{s}^{-1}\right) \\ 8.97 \pm 3.69 & 208.99 \pm 10.81\end{array}$

$2.33 \pm 0.29$

$18.36 \pm 1.16$

$\mathrm{K}_{\mathrm{d}}(\mathrm{nM})$

K260A R261

$7.88 \pm 1.48$

Figure 8.1: Electrostatic and hydrophobic contributions to KIHsv2 membrane binding. Averaged time courses of dansyl emission at different vesicle concentrations. LUVs containing (A) DOPC:DOPE:PtdIns3P:dansyl-DOPE (73:20:2:5, molar ratio) and (B) DOPC:DOPE:DOPS:dansyl-DOPE (35:40:20:5, molar ratio) were rapidly mixed with an equal volume of solutions containing KlHsv2 $(2 \mu \mathrm{M})$. Solid lines show mono-exponential fits. (C) Dependency of kobs on vesicle concentration in the presence of vesicles containing DOPC:DOPE:PI3P:dansyl-DOPE (squares) and DOPC:DOPE:DOPS:dansyl-DOPE (circles). Error bars indicate the SEM (n = 3-4). (D) Liposome flotation assays with KIHsv2 loop 6CD mutants. SUVs consisted of PC:PE:Texas-Red-PE:PI (74:23:2:1, weight ratio).(E) Summary of determined rate constants from kinetic experiments. Numbers are given taking a vesicle as a ligand unit. The experiments reported in (A)-(C) and (E) were performed by Dr. Ángel Perez Lara, while the experiments presented in (D) were performed by Dr. Karin Kühnel and Dr. Ricarda Busse.

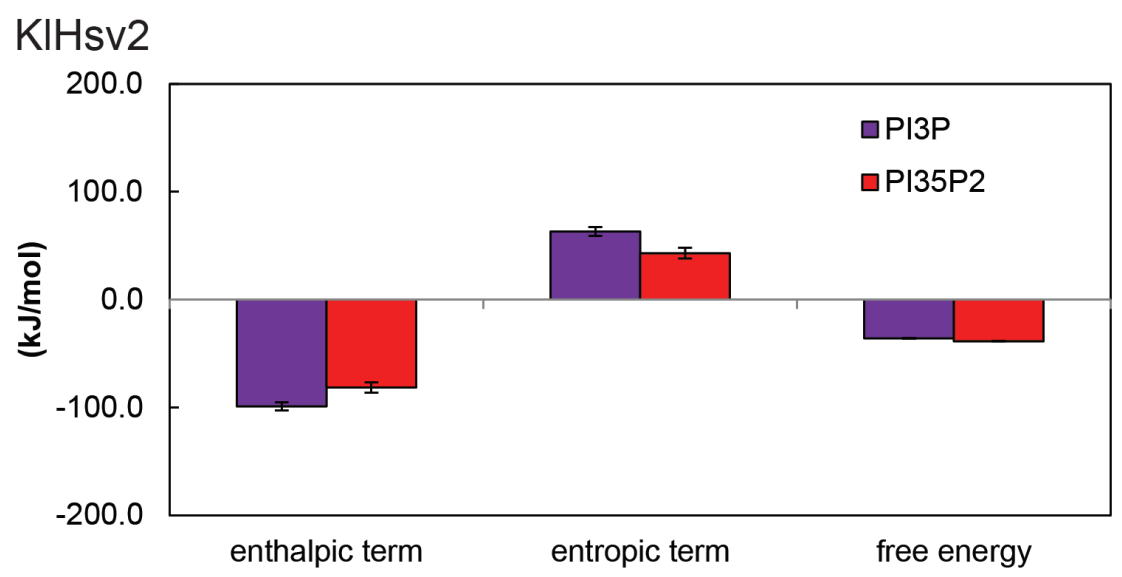

Figure 8.2: ITC measurements of KIHsv2 with liposomes containing PI3P or PI(3,5) $\mathbf{P}_{2}$. The entalphic, entropic and energy terms are represented through histograms for both PI3P and PI(3,5) $\mathrm{P}_{2}$. Error bars represent standard errors of the mean (SEM). These experiments were performed by Dr. Ricarda Busse [192]. 

Table 8.1: All contacts, salt-bridges or hydrogen bonds, revealed during the docking experiment for PI3P in binding site 1 of KlHsv2.

\begin{tabular}{|c|c|c|c|c|c|c|c|c|c|c|c|c|c|c|c|c|}
\hline & \multirow{3}{*}{ cluster } & \multirow{3}{*}{$\begin{array}{l}\text { number } \\
\text { configura- } \\
\text { tions in } \\
\text { cluster }\end{array}$} & \multirow{3}{*}{$\begin{array}{c}\text { Energy } \\
\text { (kcallmal) }\end{array}$} & \multicolumn{12}{|c|}{ Hydrogen bonds and salt bridges } & \multirow{3}{*}{ Other interactions } \\
\hline & & & & \multicolumn{4}{|c|}{$\mathrm{PO}^{2}{ }^{2}-1$} & \multicolumn{4}{|c|}{$\mathrm{PO}_{4}^{3} 3$} & \multirow{2}{*}{$\begin{array}{l}\mathrm{OH} 5 \\
\mathrm{O} 5\end{array}$} & \multirow{2}{*}{$\begin{array}{c}\mathrm{OH} 2 \\
\mathrm{O} 2\end{array}$} & \multirow{2}{*}{$\begin{array}{c}\mathrm{OH} 4 \\
\mathrm{O} 4\end{array}$} & \multirow{2}{*}{$\begin{array}{c}\mathrm{OH} 6 \\
\mathrm{O} 6\end{array}$} & \\
\hline & & & & 01 & 011 & 012 & 013 & 03 & 031 & 032 & 033 & & & & & \\
\hline \multirow{6}{*}{ frame0 } & $1-1$ & 3 & -2.86 & & & & & & & & & $\begin{array}{l}\text { A179(N), N180(OD1), } \\
\text { R205(NH1) }\end{array}$ & & A179(N) & N180(OD1) & 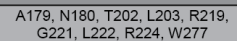 \\
\hline & $2 \_1$ & 6 & -2.51 & & & & & N180(ND2) & & R224(NH1) & & & $\begin{array}{l}\text { N180(OD1, } \\
\text { ND2) }\end{array}$ & $\begin{array}{ll}\text { T202201061); } \\
\text { R2224(N2) }\end{array}$ & & H178, A179, L203, R205 \\
\hline & $3 \_1$ & 1 & -2.31 & & & & & & & & 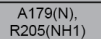 & K176(O), A177(O) & & K178(O), $A 177(0)$ & & R205, L214, V215, R219, Q274, \\
\hline & 4 4_1 & 3 & -2.14 & & & & & $\mathrm{R} 224(\mathrm{NH} 2)$ & & & & G221(0) & & L2222(O), R224(NH2) & & T202, L203, R205, L214, E217, \\
\hline & $5 \_1$ & 1 & -2.12 & & & & & & $\begin{array}{l}\begin{array}{c}\mathrm{A} 179(\mathrm{~N}), \\
\mathrm{R} 205(\mathrm{NH} 1)\end{array} \\
\end{array}$ & & R205(NH2) & & $\begin{array}{l}\text { N180(OD1, } \\
\text { ND2) }\end{array}$ & & & 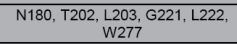 \\
\hline & $6-1$ & 2 & -2.03 & & & & & & & & 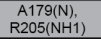 & & N180(OD1) & & & $\begin{array}{l}\text { T202, L203, R219, L222, R224, } \\
\text { W277 }\end{array}$ \\
\hline \multirow{6}{*}{ frame5 } & $1-1$ & 15 & -4.09 & & & $\mathrm{R} 219(\mathrm{NH} 2)$ & & & & R224(NH2) & & R224(N) & & & 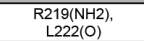 & 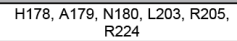 \\
\hline & $2 \_1$ & 4 & -3.18 & & & R205(NH2) & & $\mathrm{R} 219(\mathrm{NH} 2)$ & & & R219(NH2) & T202(OG1) & & 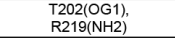 & R205(NH1) & K176, A177, A179, L203, F207 \\
\hline & $3 \_1$ & 5 & -3.13 & & R219(NH1) & & & R205(NH2) & $\begin{array}{l}\text { A179(N), } \\
\text { R205(NH1) }\end{array}$ & & & S198(OG), D200(OD1) & & H178(ND1) & $\begin{array}{l}\mathrm{D} 200(\mathrm{OD} 2), \\
\mathrm{T} 202(\mathrm{OC11})\end{array}$ & D200, L203, D223, R224 \\
\hline & 4 4-1 & 8 & -3.01 & & & & & R224(NE) & & R224(NH2) & & R219(NH2), L222(O) & & R224(N) & $\begin{array}{l}\text { T202(061), } \\
\text { R219(N-1) }\end{array}$ & $\begin{array}{l}\text { A179, D200, L203, R205, R219, } \\
\text { R224, E276, }\end{array}$ \\
\hline & $5-1$ & 1 & -2.88 & & R219(NH1) & & & $\mathrm{R} 205(\mathrm{NH} 1)$ & R205(NH2) & & & 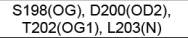 & R205(NH2) & H178(ND1) & T202(OG1) & D200, L203, D223, R224 \\
\hline & $6 \_1$ & 4 & -2.83 & & & & & R224(NE) & & & R224(NH2) & T202(OG1), L222(O) & R224(NE) & R224(N) & T202(OG1) & D200, R205, R219, R224 \\
\hline \multirow{6}{*}{ frame10 } & $1 \_1$ & 4 & -3.30 & 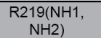 & & & & & $\begin{array}{l}\text { A1799(N)! } \\
\text { R205(N1) }\end{array}$ & & & H178(ND1), S198(OG) & R219(NH2) & A179(N), N180(N) & $\begin{array}{l}\text { D200(001) } \\
T \text { T2020(01) }\end{array}$ & D200, L203, L222, D223, R224 \\
\hline & $2 \_1$ & 5 & -3.13 & & \begin{tabular}{|l|l|}
$\mathrm{R} 205(\mathrm{NH} 1$, \\
$\mathrm{NH} 2 \mathrm{l}$
\end{tabular} & & & & R219(NH1) & & R219(NH2) & $\mathrm{s} 198(0 G)$ & & D200(OD1) & $\begin{array}{l}\text { H178 (ND1), } \\
\text { A179(N) }\end{array}$ & $\begin{array}{l}\text { H178, A179, N180, L203, R205, } \\
\text { E217, R219, L222, F F 2733 }\end{array}$ \\
\hline & $3 \_1$ & 1 & -3.01 & & R219(NH2) & & & & & & R205(NH1) & E217(OE2) & & $\begin{array}{l}\text { R205(NH2), } \\
\text { E217(OE2) }\end{array}$ & R219(NE, NH2) & $\begin{array}{l}\mathrm{N} 180,0200, \text { L203, R219, L222, } \\
\text { D223 }\end{array}$ \\
\hline & $4-1$ & 5 & -2.50 & & & & & & & & & H178(ND1), S198(OG) & & $\begin{array}{l}\text { T2020(OGG); } \\
\text { R219(NH1) }\end{array}$ & $\begin{array}{l}\text { H1188(ND)i, } \\
\text { A179(N) N180(N) }\end{array}$ & K176, H178, A179, L203, R205 \\
\hline & $5-1$ & 2 & -2.48 & R219(NH2) & & & & & & & $\begin{array}{l}A 179(\mathrm{~N}), \\
\mathrm{R} 209(\mathrm{NH} 1)\end{array}$ & S198(OG), D200(OD1) & & $\mathrm{N} 180(\mathrm{~N})$ & D200(OD1) & N180, R219, L222 \\
\hline & $6 \_1$ & 4 & -2.40 & & & & & $\begin{array}{l}\mathrm{R} 205 \mathrm{~N}(\mathrm{NH} 1, \\
\mathrm{NH2}\end{array}$ & & & $A 179(N)$ & & & A179(N) & R219(NH2) & L203, E217, F273, 0274 \\
\hline \multirow{6}{*}{ frame15 } & $11_{1}$ & 5 & -3.56 & & & & & & & $\begin{array}{l}\text { A A779(N), } \\
\text { R205(NH1) }\end{array}$ & $\mathrm{S} 198(\mathrm{OG})$ & T202(OG1) & R205(NH2) & 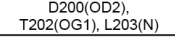 & & L203, R205, E217, R219, E276 \\
\hline & $2-1$ & 2 & -3.16 & & & $\mathrm{~W}_{277(\mathrm{~N})}$ & & & & & & E276(OE2) & & & & 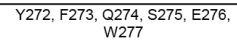 \\
\hline & $3-1$ & 8 & -2.30 & & & & & & R205(NH2) & H178(ND1) & $A 179(N)$ & & 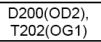 & & & 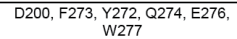 \\
\hline & $4 \_1$ & 1 & -2.12 & & & & & & & & R220(NH2) & & & & E276(OE2) & $\begin{array}{l}R 219, \text { Y272, F273, S275, E276, } \\
\text { WW77 }\end{array}$ \\
\hline & 5_1 & 1 & -2.09 & & & & & & R205(NH1) & $\begin{array}{l}\text { H178(ND), } \\
\text { S198(OG) }\end{array}$ & N180(N) & R224(NH2) & $\begin{array}{l}\text { D200(OD2), } \\
T \text { T202(OC1) }\end{array}$ & & & D200, L203, E217, R219, E276 \\
\hline & $6 \_1$ & 3 & -2.05 & & & & & & & & & & & E217(OE1) & & Y272. F273, S275, E276, W277 \\
\hline \multirow{6}{*}{ frame20 } & $1 \_1$ & 30 & -3.83 & & & & & R205(NH2) & $\begin{array}{l}\text { A179(N), } \\
\text { R205(NH1) }\end{array}$ & & & $D 200(0 D 1)$ & & & $\begin{array}{l}\text { T202201011) } \\
\text { R219(N-1) }\end{array}$ & L203, E217, R219, G221, E276 \\
\hline & $2-1$ & 1 & -2.80 & & R205(NH2) & $\begin{array}{l}\begin{array}{r}1 A 77(7) \\
\text { R205(NH1) }\end{array} \\
\end{array}$ & & & & & & $\mathrm{N} 180(0), 0200(002)$ & & S198(OG), D200(OD 1) & $A 179(\mathrm{~N}), \mathrm{N} 180(\mathrm{~N})$ & 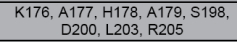 \\
\hline & $3 \_1$ & 7 & -1.91 & & & & & & H178(ND1) & $\mathrm{N} 180(\mathrm{~N})$ & 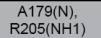 & & & & & L203, E217, R219, , 2274, E276 \\
\hline & $4 \_1$ & 5 & -1.80 & & & & & & $\begin{array}{l}\begin{array}{l}147(7)(N), 1 \\
R 205(N-1)\end{array} \\
\end{array}$ & & R205(NH2) & D200(OD2) & R205(NH2) & & & A179, L203, R219, ,2274, E276 \\
\hline & $5 \_1$ & 6 & -1.72 & & & & & & & & & $E 217(0)$ & E276(OE1) & Q274(0) & E217(OE1) & 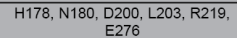 \\
\hline & $6-1$ & 5 & -1.61 & & & R205(NH2) & & & & & & E217(0) & & E276(OE1) & E217(OE1) & A179, D200, L203, 205, R219, \\
\hline \multirow{6}{*}{ frame25 } & $11_{1}$ & 2 & -2.41 & & & & & & R205(NH2) & & R205(NH1) & $k 176(0)$ & & $A 177(0)$ & & R205, L214, R219, F273, Q274 \\
\hline & $2 \_1$ & 3 & -1.96 & & & & & $\mathrm{R} 205(\mathrm{NH} 1)$ & & $\mathrm{R} 205(\mathrm{NH} 2)$ & & H178(NE1) & R205(NH2) & & & T202, L203, R219 \\
\hline & $3-1$ & 5 & -1.87 & & & & & & R205(NH2) & & R205(NH1) & $\mathrm{K} 176(0)$ & & & & K176, A177, F207, V213, F273 \\
\hline & $4-1$ & 4 & -1.82 & R219(NH2) & & & & & & & R205(NH2) & & 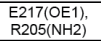 & & & $R 219$, F273, Q274 \\
\hline & $5 \_1$ & 4 & -1.79 & & & & & & $\mathrm{R} 219(\mathrm{NH} 2)$ & & & Q274(NE2) & E217(OEE1) & $\mathrm{R} 219(\mathrm{NH} 2)$ & & $\begin{array}{r}\text { K176, A177, A179, F2077, L214, } \\
\text { E217, Q274 }\end{array}$ \\
\hline & $6 \_1$ & 3 & -1.66 & & & & & & & & & E217(OE2), Q274(NE2) & & & & $\begin{array}{l}\text { K176, A177, } F 207, \text { V213, L214, } \\
\text { F273 }\end{array}$ \\
\hline \multirow{6}{*}{ frame30 } & $1-1$ & 6 & -1.61 & & & & & & & R219(NH2) & & T202(OG 1), L2222(0) & & L.222(0) & T202(OG 1) & $\mathrm{N} 180$, L203 \\
\hline & $2-1$ & 1 & -1.31 & N180(ND2) & & $\begin{array}{c}\mathrm{R} 2055 \mathrm{NH} 1, \\
\mathrm{NH2}\end{array}$ & N180(ND2) & & & & & & $\begin{array}{l}2000(002), \\
T 202(001)\end{array}$ & L2222(0) & & $\mathrm{N} 180, \mathrm{~L} 203$ \\
\hline & 3_1 & 4 & -1.28 & & & & & N180(ND2) & N180(ND2) & & R205(NH1) & D200(OD 1), T202(OG 1) & & $\begin{array}{l}\text { N180(ND2); } \\
\text { D200(OD2) }\end{array}$ & & L203, R219, L222 \\
\hline & $4 \_1$ & 3 & -1.16 & & N180(ND2) & & & & & & R219(NH2) & T202(OG1) & & R219(NH2) & T202(OG1) & A179, N180, L203, R205 \\
\hline & $5 \_1$ & 1 & -1.08 & & & & & & N180(ND2) & & & & & & & $\begin{array}{c}200, \text { L203, R205, R219, L222, } \\
\text { D2233, R244, }\end{array}$ \\
\hline & 6_1 & 10 & -0.81 & R219(NH2) & R219(NH1) & & & & & R205(NH1) & & & & & R219(NH1) & T202, L203, R219, L2222 \\
\hline
\end{tabular}


Table 8.2: All contacts, salt-bridges or hydrogen bonds, revealed during the docking experiment for $\mathrm{PI}(3,5) \mathrm{P}_{2}$ in binding site 1 of $\mathrm{KlHsv} 2$.

\begin{tabular}{|c|c|c|c|c|c|c|c|c|c|c|c|c|c|c|c|c|c|c|c|}
\hline & \multirow{3}{*}{ cluster } & \multirow{3}{*}{$\begin{array}{c}\text { number } \\
\text { configurarions } \\
\text { in cluster }\end{array}$} & \multirow{3}{*}{ 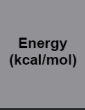 } & \multicolumn{12}{|c|}{ Hydrogen bonds and salt bridges } & \multirow{3}{*}{$\begin{array}{l}\mathrm{OH} 2 \\
\mathrm{O} 2 \\
\end{array}$} & \multirow{3}{*}{$\begin{array}{c}\mathrm{OH} 4 \\
\mathrm{O} 4\end{array}$} & \multirow{3}{*}{$\begin{array}{c}\text { OH } 6 \\
\text { O6 }\end{array}$} & \multirow{3}{*}{ Other interactions } \\
\hline & & & & \multicolumn{4}{|c|}{$\mathrm{PO}^{\mathrm{A}^{2}-1}$} & \multicolumn{4}{|c|}{$\mathrm{PO}_{4}^{3} 3$} & \multicolumn{4}{|c|}{$\mathrm{PO}_{4}^{2-5}$} & & & & \\
\hline & & & & 01 & 011 & 012 & 013 & 03 & 031 & 032 & 033 & 05 & 051 & 052 & 053 & & & & \\
\hline \multirow{6}{*}{ frameo } & $1-1$ & 12 & -3.18 & & & & & & R205(NH2) & $\begin{array}{l}A 279(\mathrm{~N}) \\
\mathrm{R} 205(\mathrm{NH})\end{array}$ & & & & $\mathrm{R} 224(\mathrm{NH} 1)$ & N180(ND2) & N180(OD1) & & & T202, R219, G221, L222 \\
\hline & 2,1 & 11 & -2.77 & & & & & R205(NH2) & & $\begin{array}{l}A 179(\mathrm{~N}): \\
\mathrm{R} 205 \mathrm{NH})\end{array}$ & & & & & $\begin{array}{l}\mathrm{N} 180(\mathrm{NN} 2), \\
\mathrm{R} 224 \mathrm{NH} 2)\end{array}$ & R205(NH2) & N180(OD1) & & $\begin{array}{l}\text { T202, L203, R219, L222. } \\
\text { E } 2776 \text { W277 }\end{array}$ \\
\hline & $3-1$ & 2 & -2.68 & & & & & & & R205(NH2) & $\begin{array}{l}A 179(N) \\
R 20(N 1)\end{array}$ & & N180(ND2) & & R224(N-1) & & & & N180, L203, R219, L2222 \\
\hline & $4 \_1$ & 4 & -2.47 & & & & & & & R205(NH2) & $\begin{array}{c}A 179(\mathrm{~N}) \mathbf{1} \\
\mathrm{R} 205(\mathrm{NH} 1)\end{array}$ & N180(ND2) & & & R224(NH1) & N180(OD1) & & & $\begin{array}{ll}\text { N180, T202, 2219, G221 } \\
\text { L2222,W27 }\end{array}$ \\
\hline & $55_{-1}$ & 9 & -1.89 & & $\begin{array}{l}A 179(\mathrm{~N}) \\
\text { R205(NH1) }\end{array}$ & R205(NH2) & & & R224(NH2) & & & & & & & N180(OD1) & & & 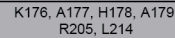 \\
\hline & $6 \_1$ & 6 & -1.88 & & A179(N) & $\begin{array}{l}A 179(\mathrm{~N}) \\
\mathrm{R} 205(\mathrm{NH})\end{array}$ & $\begin{array}{ll}R 205(\mathrm{NH} 1 . \\
\mathrm{NH2}\end{array}$ & & & & & & & & N180(ND2) & & & N180(OD1) & $\begin{array}{l}\text { L203, R205, F207, L214 } \\
\text { E217, }\end{array}$ \\
\hline \multirow{6}{*}{ frame5 } & $1 \_1$ & 8 & -4.36 & & & $\begin{array}{ll}H 1788(N D D), \\
N\end{array}$ & R205(NH1) & R219(NH2) & & & & & & R224(NE) & & & & $\begin{array}{l}\text { N180(ND2), } \\
\text { D200(OOD1) }\end{array}$ & $\begin{array}{ll}\text { K176, A177, A179, D200 } \\
, 202,527\end{array}$ \\
\hline & $2-1$ & 9 & -3.79 & R219(NH2) & & & & & & R224(NH2) & R224(NE) & & & $\begin{array}{l}\text { H1788(NDD), } \\
\text { N } 180(\mathrm{~N})\end{array}$ & R205(N-1) & & & & E217, R219, E276 \\
\hline & $3-1$ & 6 & -3.55 & & & $\begin{array}{l}\text { H1788(ND1), } \\
\text { N180(N) }\end{array}$ & R205(NH1) & R219(NH2) & & $\begin{array}{ll}\text { R219(NH1, } \\
\text { NH2) }\end{array}$ & & & & $\mathrm{R} 224(\mathrm{NH} 2)$ & R224(NE) & & & $\begin{array}{l}\text { N180(ND2) } \\
\text { D200(OD1) }\end{array}$ & $\begin{array}{c}\text { A177, A179, 2000, R205 } \\
\text { E217, }\end{array}$ \\
\hline & $4-1$ & 5 & -3.53 & & & & & & & & R219(NH1) & & & R224(NE) & R224(N-2) & & & & 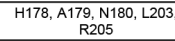 \\
\hline & 5 & 3 & -2.99 & R205(NH1) & \begin{tabular}{|l|} 
H178(ND) \\
Ni80(N)
\end{tabular} & & & & & & R205(NH2) & & & & R219(NH1) & & & & 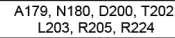 \\
\hline & $6 \_1$ & 8 & -2.47 & & H178(ND1) & $\begin{array}{ll}\mathrm{R} 205(\mathrm{NH} 1, \\
\mathrm{NH2}\end{array}$ & & & & R219(NH1) & & & & R224(NH2) & R224(NE) & & & 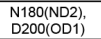 & A179, N180, D200, R205 \\
\hline \multirow{6}{*}{ frame10 } & $1-1$ & 18 & -3.84 & H178(ND1) & $5198(06)$ & N180(N) & & & & R205(NH2) & A179(N) & & & R219(NH2) & & $A 179(N)$ & & R219(NH2) & 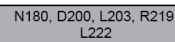 \\
\hline & $2 \_1$ & 10 & -3.31 & & & & & R219(NH2) & & & R219(NE) & & & R205(NH2) & $A 179(N)$ & R219(NH2) & & & 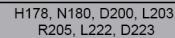 \\
\hline & $3-1$ & 10 & -2.66 & & & & & & R219(NH2) & R219(NE) & & & & R205(NH+2) & & $\mathrm{R} 219(\mathrm{NH}+2)$ & R205(NH2) & & $\mathrm{N} 180, \mathrm{D} 200$, , L203, D223 \\
\hline & $4-1$ & 6 & -2.48 & & & & & & & $\begin{array}{l}\text { R205(NH1, } \\
\text { NH2) }\end{array}$ & $\begin{array}{c}A 179(\mathrm{~N}) \\
\mathrm{R} 205(\mathrm{NH} 1)\end{array}$ & & & & & & & & $\begin{array}{c}L 203, R 219, F 273,0274 \\
E 2766\end{array}$ \\
\hline & $5-1$ & 1 & -1.85 & & & $\mathrm{R} 219(\mathrm{NH} 2)$ & & & & & & & & $\mathrm{N} 180(\mathrm{~N})$ & $\mathrm{S} 198(0 \mathrm{OG})$ & & $A 179(N)$ & R219(NH2) & L203, R205, E217 \\
\hline & $6-1$ & 1 & -1.74 & & R219(NH2) & & & & & & & $A 179(N)$ & & $\mathrm{R} 205(\mathrm{NH} 2)$ & & & & H178(ND1) & $\begin{array}{l}\text { A179, L203, } 2217, \text {, R219, } \\
\text { L222 }\end{array}$ \\
\hline \multirow{6}{*}{ frame15 } & $1 \_1$ & 2 & -3.39 & & & & & & & & R220(NH2) & & & & & & R220(NH1) & E276(OE2) & 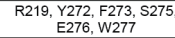 \\
\hline & $2 \_1$ & 4 & -2.23 & & & & & & & & & & S198(OG) & $\mathrm{N} 180(\mathrm{~N})$ & $\begin{array}{c}A 179(\mathrm{~N}), \\
\mathrm{R} 205(\mathrm{NH})\end{array}$ & & D200(OD2) & R205(NH2) & 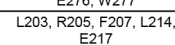 \\
\hline & $3 \_1$ & 9 & -1.97 & & & R205(NH2) & & & & & $\begin{array}{c}\begin{array}{c}\text { R219(NE, } \\
\mathrm{N}+22)\end{array} \\
\end{array}$ & & $\begin{array}{l}1178(\mathrm{ND} 1), \\
\text { S198(OG) }\end{array}$ & $\mathrm{N} 180(\mathrm{~N})$ & $\begin{array}{l}\text { A19(N) } \\
\text { R205(NH1) }\end{array}$ & & & R200(NH2) & $\begin{array}{c}L 203, L 214, E 217, F 273 \\
E E 276\end{array}$ \\
\hline & $4 \_1$ & 2 & -1.43 & & & $\mathrm{R} 205(\mathrm{NH} 2)$ & & & & & & & $\mathrm{S} 198(\mathrm{OG})$ & $\mathrm{N} 180(\mathrm{~N})$ & $\begin{array}{l}\begin{array}{c}A 19(N) \\
\text { R205(NH1) }\end{array} \\
\end{array}$ & & $\mathrm{D} 200(\mathrm{OD} 2)$ & R205(NH2) & $\llcorner 203$, E217, ₹273, Q274 \\
\hline & $5-1$ & 1 & -1.12 & & R219(NH2) & & & & R205(NH2) & & & & $\mathrm{A} 179(\mathrm{~N})$ & H178(ND1) & $\mathrm{S} 198(\mathrm{OG})$ & R205(NH2) & & & 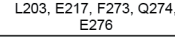 \\
\hline & $6 \_1$ & 3 & -1.00 & & & & & & H178(ND1) & $\mathrm{S} 198(\mathrm{OG})$ & $\begin{array}{c}1179(\mathrm{~N}) \\
\mathrm{R} 205(\mathrm{NH}) \\
\end{array}$ & & & & 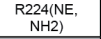 & $\mathrm{D} 200(\mathrm{OO} 2)$ & & & L203, R205, Е217, Е276 \\
\hline \multirow{6}{*}{ frame20 } & $1-1$ & 22 & -2.16 & & R219(NH2) & & & & & $\mathrm{R} 205(\mathrm{NH} 2)$ & & & & $\begin{array}{l}N 179(\mathrm{Ni}) \\
\mathrm{R} 205(\mathrm{NH})\end{array}$ & $\mathrm{H} 178(\mathrm{ND1})$ & & & & L203, R219, E276 \\
\hline & 2 -1 & 10 & -1.76 & & R219(NE) & R219(NH2) & & & H178(ND1) & $\mathrm{N} 180(\mathrm{~N})$ & R205(NH1) & & R224(NH2) & R224(NH2) & & & & & $\begin{array}{c}\text { D200, L203, E217, R219, } \\
E 2766\end{array}$ \\
\hline & 3_1 & 9 & -1.65 & & & & & R219(NH1) & & R224(NH2) & $\begin{array}{c}\mathrm{R} 219(\mathrm{NH} 1, \\
\mathrm{NH2} 2\end{array}$ & & $\begin{array}{l}\begin{array}{l}\mathrm{N} 179(\mathrm{~N}) \\
\text { N180(N) }\end{array} \\
\end{array}$ & & $\begin{array}{c}\begin{array}{c}\mathrm{R} 205(\mathrm{NH} 1, \\
\mathrm{NH} 2)\end{array} \\
\end{array}$ & & & & $\begin{array}{c}\text { D200, L203, R219, Q274 } \\
\text { E276 }\end{array}$ \\
\hline & $4 \_1$ & 15 & -1.47 & & R205(NH2) & & & & & & & & R219(NE) & & R219(NH2) & & & & $\begin{array}{l}H 178, \text { A A179, N180, D200 } \\
\text { L203, R205, R219 }\end{array}$ \\
\hline & $5 \_1$ & 3 & -1.40 & & & & & & 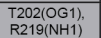 & R219(NH2) & R224(NH2) & & $\mathrm{N} 180(\mathrm{~N})$ & $\begin{array}{c}\mathrm{N} 179(\mathrm{~N}) \\
\mathrm{R} 205(\mathrm{NH})\end{array}$ & $\mathrm{H} 178(\mathrm{ND} 1)$ & & D200(002) & & D200, L203, R205, R219 \\
\hline & $6 \_1$ & 4 & -1.09 & & & R205(NH2) & & & & & & & $\begin{array}{c}\begin{array}{c}\mathrm{A} 179(\mathrm{~N}) \\
\mathrm{R} 205(\mathrm{NH} 1)\end{array} \\
\end{array}$ & $\begin{array}{l}\text { H1788(ND), } \\
\text { N180(N) }\end{array}$ & & R205(NH2) & & & L203, R219, E276 \\
\hline \multirow{6}{*}{ frame25 } & $1-1$ & 1 & -1.61 & & R205(NH2) & & & & & & & & & R205(NH1) & R205(NH1) & $\mathrm{A} 177(0)$ & & & $\begin{array}{l}\text { R205, LL14, E217, R219, } \\
\text { F273, }, 2274\end{array}$ \\
\hline & $2 \_1$ & 4 & -1.59 & & & & & R205(NH2) & & R205(NH1) & & & & & & E217(OE1) & & & L214, E217, F273, Q274 \\
\hline & 3_1 & 1 & -1.54 & & & Q274(NE2) & & R219(NH2) & & R219(NE) & & & R219(NH2) & & & R219(NH2) & & & 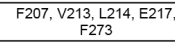 \\
\hline & $4{ }^{4} 1$ & 3 & -1.50 & & & & & & & & Q274(N) & & R205(NH2) & & R205(NH2) & & $\begin{array}{l}\text { E217(OE1), } \\
\text { R219(NH2) }\end{array}$ & & $\begin{array}{l}\text { K176, FF207, V213, L214 } \\
E 2217,0274\end{array}$ \\
\hline & 5 -1 & 4 & -1.36 & & & & & & $\begin{array}{l}\begin{array}{l}A 179(N), \\
\text { N180(N) }\end{array} \\
\end{array}$ & R205(NH1) & R205(NH1) & & & & & & & K176(O) & $\mathrm{A} 1777, \mathrm{~V} 213, \mathrm{~L} 214, \mathrm{~F} 273$ \\
\hline & $6 \_1$ & 6 & -1.29 & & & & & & & & & & & & R205(NH2) & & $\begin{array}{l}\text { E217(OE1), } \\
\text { R219(NH2) }\end{array}$ & & 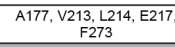 \\
\hline & $1-1$ & 15 & -1.64 & & & $\mathrm{R} 219(\mathrm{NH} 2)$ & & & & & R219(NH1) & & N180(ND2) & & R205(NH1) & & & & T202, R219, L222, D223 \\
\hline & $2-1$ & 10 & -1.45 & & & & & & N180(ND2) & & R205(NH2) & & & $\mathrm{R} 219(\mathrm{NH} 2)$ & & & R205(NH2) & R219(NH2) & D200, L203, D223, R224 \\
\hline frame30 & $3 \_1$ & 7 & -1.36 & & & & & R205(NH2) & & & R205(NH1) & & R219(NH1) & & R219(NH2) & & & & $\begin{array}{l}\text { D200, L203, R219, L2222 } \\
\text { D2233, R224 }\end{array}$ \\
\hline Irameso & $4-1$ & 4 & -0.83 & & & & & & & $\mathrm{R} 205(\mathrm{NH} 2)$ & R205(NH1) & & $R 219(\mathrm{NH} 1)$ & & R219(NH2) & $\mathrm{R} 219(\mathrm{NH} 2)$ & & & L203, L222, D2233 \\
\hline & $5-1$ & 2 & -0.58 & & & $\mathrm{R} 219(\mathrm{NH} 2)$ & & & & & 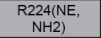 & N180(ND2) & R205(NH1) & & & & $\begin{array}{l}\text { N } 180\left(\mathrm{ND}_{212}\right) \\
\text { D200(OOD2) }\end{array}$ & & D200, L203, L222, D223 \\
\hline & $6-1$ & 3 & -0.52 & & & & & & & $\begin{array}{l}\mathrm{R} 219 \mathrm{NH} 1, \\
\mathrm{NH2}\end{array}$ & & & & & $\mathrm{N} 180(\mathrm{~N})$ & & & N180(ND2) & L203, L2222, R224 \\
\hline
\end{tabular}


Table 8.3: All contacts, salt-bridges or hydrogen bonds, revealed during the docking experiment for PI3P in binding site 2 of KlHsv2.

\begin{tabular}{|c|c|c|c|c|c|c|c|c|c|c|c|c|c|c|c|c|}
\hline & \multirow{3}{*}{ cluster } & \multirow{3}{*}{$\begin{array}{c}\text { number } \\
\text { confifurations } \\
\text { in cluster }\end{array}$} & \multirow{3}{*}{$\begin{array}{l}\text { Energy } \\
\text { (kcallmol) }\end{array}$} & \multicolumn{12}{|c|}{ Hydrogen bonds and salt bridges } & \multirow{3}{*}{ Other interactions } \\
\hline & & & & & $\mathrm{PO}_{4}$ & & & & & $a^{3} 3$ & & OH 5 & OH 2 & OH 4 & ОН 6 & \\
\hline & & & & 01 & 011 & 012 & 013 & 03 & 031 & 032 & 033 & 05 & 02 & 04 & 06 & \\
\hline \multirow{6}{*}{ frameo } & $1-1$ & 1 & -3.39 & & & & & & & W246(NE1) & K288(NZ) & N291(O) & & $\mathrm{N} 291(0)$ & $\mathrm{s} 226(\mathrm{OG})$ & K183, R224, S226, 1228, D244 \\
\hline & $2 \_1$ & 4 & -2.79 & & & & & & & & & D244(0), W246(NE1) & & 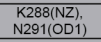 & $\mathrm{D} 244(0)$ & R224, T225, S226, D244 \\
\hline & $3-1$ & 1 & -2.73 & & & & & & & & K288(NZ) & D244(0) & W246(NE1) & & & K183, D244, 1228, D292 \\
\hline & $4-1$ & 1 & -2.48 & & & & & & & & K183(NZ) & R224(0) & & Q199(0) & & S226, K245, W246, K288 \\
\hline & $5 \_1$ & 3 & -2.42 & & & & & & & K288(NZ) & K288(NZ) & D244(O) & D244(0) & & D244(OD1) & R224, T225, S226, D244 \\
\hline & $6-1$ & 3 & -2.40 & & & & & K288(NZ) & & & K288(NZ) & W246(NE1) & & $\begin{array}{l}\text { K288(NZ)i } \\
\text { N291(ODDi) }\end{array}$ & D244(0) & D223, T225, K245 \\
\hline \multirow{6}{*}{ frame5 } & $1-1$ & 4 & -5.35 & & & & & & & & K245(NZ) & 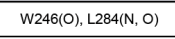 & K245(O) & $\begin{array}{ll}\text { Le284(O) } \\
\text { V286(N) }\end{array}$ & & K245, H249, К283 \\
\hline & $2 \_1$ & 1 & -4.94 & N281(ND2) & & R220(NH1) & & & & & L2222(N) & R219(O) & \begin{tabular}{|l} 
W277(NE1) \\
\end{tabular} & R219(0) & $\begin{array}{l}\text { R220(N-1), } \\
\text { S228(OGG) }\end{array}$ & R220, D244, T247, H249, W277, K283 \\
\hline & $3-1$ & 9 & -4.83 & & & & & & & & K245(NZ) & $\mathrm{V} 286(\mathrm{O}, \mathrm{N})$ & K245(O) & $\mathrm{V}_{2286(0)}$ & & $\mathrm{D} 244, \mathrm{~T} 247, \mathrm{H} 249, \mathrm{~K} 283, \mathrm{~K} 285, \mathrm{~V} 286$ \\
\hline & $4 \_1$ & 4 & -3.89 & & & & & & & & K245(NZ) & $\mathrm{W}_{246(O), L 284(\mathrm{O}, \mathrm{N})}$ & K245(O) & 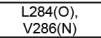 & & D244, T247, H249 \\
\hline & $55_{-1}$ & 11 & -1.68 & & & & & & & & & $w_{246(0)}$ & 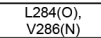 & & 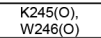 & D244, K245, K283, L284, K285 \\
\hline & $6 \_1$ & 2 & -3.73 & $\mathrm{v}_{226(\mathrm{~N})}$ & & & & & & & & W246(O), L284(N, O) & V286(N) & & 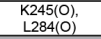 & D244, K245, L284, K2825 \\
\hline \multirow{6}{*}{ frame10 } & $11^{1}$ & 2 & -3.78 & & K245(NZ) & & K245(N) & & K245(NZ) & & & R224(O) & D244(OD2) & & R2224(0) & S2226, 1228, K245, W246 \\
\hline & $2-1$ & 1 & -3.05 & & & & & K183(NZ) & & & $\mathrm{K} 183(\mathrm{NZ})$ & Q199(0), T225(O) & K183(NZ) & T225(0) & & R224, T225, S226, 1228, D244, K245 \\
\hline & 3_-1 & 4 & -2.97 & & & & & & & & K245(NZ) & D244(0) & D244(OD2) & & D244(0) & R220, D244, K245, T247, H249, N281 \\
\hline & $4 \_1$ & 2 & -2.85 & & & & & & & & & R224(0) & & D244(OD1) & & I204, R2220, G221, T225, I227, V241, S243, D244, $\mathrm{H} 249$ \\
\hline & 5 & 12 & -2.82 & & & & & & & & K245(NZ) & & D244(OD2) & & & G221, T225, , 243, D244, K245, T247, H249, N281, F282 \\
\hline & $6 \_1$ & 3 & -2.79 & & K245(NZ) & & & & & & & & & & & T2225, S243, D244, T247, H249, N281, F282 \\
\hline \multirow{6}{*}{ frame15 } & $1-1$ & 32 & -5.47 & & & & & & & & G221(N) & T225(OG1), S243(OG) & & L2222(N) & $\begin{array}{cl}244(0) \\
T 247(0 G 1)\end{array}$ & L222, K245, H249, V286 \\
\hline & $2-1$ & 3 & -4.86 & & & & & & G221(N) & & R220(NH2) & 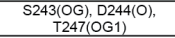 & & $\begin{array}{ll}\text { T22525(OGG), } \\
\text { S243(OGG) }\end{array}$ & $\mathrm{D} 244(0)$ & L2222, T247, H249, N281, F282, K283 \\
\hline & $3-1$ & 1 & -4.55 & & & & & G221(N) & & & R220(NH2) & T225(OG1), 5243(OG) & R220(NH2) & L2222(N) & T247(OG1) & R220, L222, H249, N281, K283 \\
\hline & $4-1$ & 5 & -4.50 & & & & & & R220(NH2) & G221(N) & & $\mathrm{T} 225(\mathrm{OG} 1), \mathrm{S} 243(\mathrm{OG})$ & & L2222(N) & 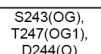 & L222, K283 \\
\hline & 5_1 & 5 & -4.19 & & & & & & & & K245(NZ) & K245(O) & D244(0) & & & L222, K245, Т247, Ғ282, K283 \\
\hline & $6 \_1$ & 4 & -4.03 & & & & & & & & K283(NZ) & L284(0) & & & K245(0) & R220, L222, T247, H249, N281, F282, K283 \\
\hline \multirow{6}{*}{ frame20 } & $1-1$ & 12 & -4.76 & & & & & & & & K283(NZ) & R220(NH2) & T247(OG1) & R220(NH2) & $\mathrm{D} 244(\mathrm{OD} 1)$ & L222, D223, R224, T225, K245, T247, \\
\hline & $2-1$ & 1 & -4.01 & & & & & & & & & D244(001, 0) & & T247(OG1) & R220(NE) & R220, G221, L222, T247, W277, N281 \\
\hline & $3-1$ & 5 & -4.00 & R220(NH2) & & R220(NH2) & & & K283(NZ) & & K283(NZ) & D244(0). T247(OG1) & & & R220(NH2) & R220, G221, T247, W277 \\
\hline & $4{ }_{4} 1$ & 1 & -3.73 & & & & & & & K283(NZ) & K283(NZ) & 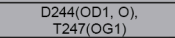 & & T247(OG1) & & L2222, R224, T225, D244, K245, T247 \\
\hline & $5 \_1$ & 4 & -3.60 & & & & & & & & K283(NZ) & & T247(OG1) & R220(NH2) & $\mathrm{D} 244(\mathrm{OD} 1)$ & L2222, K245, T247, K283 \\
\hline & $6-1$ & 5 & -3.49 & & & & & & & & K283(NZ) & D244(001, 0) & T247(OG1) & R220(NH2) & & L2222, K245, T247 \\
\hline \multirow{6}{*}{ frame25 } & $1-1$ & 9 & -5.47 & & & & & & K283(NZ) & & K283(NZ) & $\mathrm{T} 247(0 \mathrm{G} 1)$ & & D244(0) & H249(NE2) & R219, R220, G221, L222, T247, H249, E276, N281 \\
\hline & $2 \_1$ & 1 & -4.48 & & & & & & 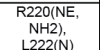 & & & E276(O), s278(OG) & & $\begin{array}{l}\text { R220(NE), } \\
\text { G221(N), }\end{array}$ & 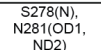 & T247, L248, H249, N281, F282 \\
\hline & $3-1$ & 8 & -4.38 & & H249(NE2) & T247(OG1) & & & & & K283(NZ) & & & & & R222, G221, L222, D244, T247, H249 \\
\hline & $4 \_1$ & 10 & -4.37 & H249(NE2) & & R220(NH2) & & & K283(NZ) & & & & & & & R2220, G221, L222, H249, F 251, W277, S278 \\
\hline & $5-1$ & 3 & -4.21 & H249(NE2) & R220(NH2) & & & & & & K283(NZ) & D244(O) & & K283(NZ) & T247(OG1) & R2220, G221, L2222, T247, H249, F251 \\
\hline & $66_{-1}$ & 8 & -4.05 & & & K283(NZ) & & & & & K283(NZ) & & & & D244(0) & L2222, K245, T247, H249, N281, F282, K2833 \\
\hline \multirow{6}{*}{ frame 30} & $1 \_1$ & 4 & -4.46 & & & & & & & & K245(NZ) & 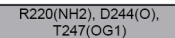 & \begin{tabular}{|l|} 
D244((OD2), \\
K 244(NZ)
\end{tabular} & $\begin{array}{l}\mathrm{D} 244(0) \\
\mathrm{K} 2450\end{array}$ & $\begin{array}{l}\text { R220(N-1); } \\
\text { T225501) }\end{array}$ & R224, T225, S226, D244, K245 \\
\hline & $2 \_1$ & 3 & -3.49 & & K245(NZ) & & & & & & K245(NZ) & 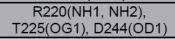 & \begin{tabular}{|l|l|}
$\mathrm{D} 244(\mathrm{OD} 2)$ \\
\end{tabular} & D244(0) & & D223, R224, D244, K245 \\
\hline & $3-1$ & 4 & -3.23 & & R220(NH2) & R220(NH2) & & & K245(NZ) & & & D223(0) R224(0) & & $R 224(0)$ & 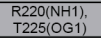 & T2225, K245, T247, H249, N281 \\
\hline & $4 \_1$ & 2 & -3.23 & & & K283(NZ) & & & & & $\mathrm{K} 245(\mathrm{NZ})$ & $\begin{array}{c}\text { R220(NH1, NH2), } \\
\text { T225(OG1) }\end{array}$ & & D244(OD2) & $\begin{array}{l}\text { R22(NNH2): } \\
\text { R244(O) }\end{array}$ & D244, K283 \\
\hline & 5 & 1 & -3.04 & & & & & & & & K283(NZ) & D244(002, 0$)$ & K245(NZ) & 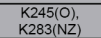 & $\mathrm{D} 244(\mathrm{OD} 2)$ & R220, G221, L2222, D2233, K245, T247 \\
\hline & $66_{-1}$ & 2 & -2.95 & & & & & & K283(NZ) & & & $\mathrm{D} 244(0)$ & & $\begin{array}{l}\text { K245(i) } \\
\text { K2233(N) }\end{array}$ & & R220, T225, K245, T247, S278 \\
\hline
\end{tabular}


Table 8.4: All contacts, salt-bridges or hydrogen bonds, revealed during the docking experiment for $\mathrm{PI}(3,5) \mathrm{P}_{2}$ in binding site 2 of $\mathrm{K} 1 \mathrm{Hsv} 2$.

\begin{tabular}{|c|c|c|c|c|c|c|c|c|c|c|c|c|c|c|c|c|c|c|c|}
\hline & \multirow{3}{*}{ cluster } & \multirow{3}{*}{$\begin{array}{c}\text { number } \\
\text { configurations } \\
\text { in culuster }\end{array}$} & \multirow{3}{*}{$\begin{array}{c}\text { Energy } \\
\text { (kcallmol) }\end{array}$} & \multicolumn{12}{|c|}{ Hydrogen bonds and salt bridges } & \multirow{3}{*}{$\begin{array}{c}\text { OH } 2 \\
\text { O2 }\end{array}$} & \multirow{3}{*}{\begin{tabular}{|l|}
$\mathrm{OH} 4$ \\
$\mathrm{O}$
\end{tabular}} & \multirow{3}{*}{$\begin{array}{c}\text { он } 6 \\
\text { о6 }\end{array}$} & \multirow{3}{*}{ Other interactions } \\
\hline & & & & \multicolumn{4}{|c|}{$\mathrm{PO}_{4}^{2-1}$} & \multicolumn{4}{|c|}{$\mathrm{PO}_{1}{ }^{2} 3$} & \multicolumn{4}{|c|}{$\mathrm{PO}_{4}^{3}-5$} & & & & \\
\hline & & & & 01 & 011 & 012 & 013 & 03 & 031 & 032 & 033 & 05 & 051 & 052 & 053 & & & & \\
\hline \multirow{6}{*}{ frameo } & $1-1$ & 2 & -3.15 & & & & & & & K288(NZ) & K288(NZ) & & & & & & & D244(001) & $\begin{array}{l}\mathrm{K} 183,0199,5226,1228, \\
0244,\end{array}$ \\
\hline & $2 \_1$ & 3 & -2.68 & & & & & & & & & & K288(NZ) & & K288(NZ) & & N291(001) & $\mathrm{D} 244(0)$ & 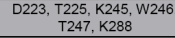 \\
\hline & $3-1$ & 9 & -1.89 & & & & & W246(NE1) & & & & & & & & D244(0) & & & D223, R224, T225, W246 \\
\hline & $4-1$ & 3 & -1.89 & & & & & & & & & R224(N) & & & & & & & 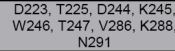 \\
\hline & $5-1$ & 9 & -1.73 & & & & & & & & & & & & K288(NZ) & & $\begin{array}{c}\begin{array}{c}244(0), \\
\text { W246(NE1) }\end{array} \\
\end{array}$ & & $\begin{array}{l}199,020, \text { R224, T225, } \\
\text { S2226, D244 }\end{array}$ \\
\hline & $6-1$ & 1 & -1.69 & & & & & & K288(NZ) & & & & & & K288(NZ) & & $\begin{array}{l}\mathrm{K} 288(\mathrm{NZZ)}) \\
\mathrm{N} 291(\mathrm{O}) 11)\end{array}$ & D244(0) & $\begin{array}{ll}0199,0200, \\
\text { S2228, W24, T225 }\end{array}$ \\
\hline \multirow{6}{*}{ frame5 } & $1-1$ & 10 & -4.23 & & $\begin{array}{l}284 \\
(\mathrm{~N}) \\
\end{array}$ & & & & & & & & & K245(NZ) & K245(NZ) & & & K245(O) & 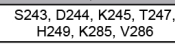 \\
\hline & 2 & 4 & -3.76 & & & & & & & & 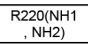 & & & & & & $\begin{array}{l}\text { N281(OD1, } \\
\text { NO2) }\end{array}$ & & 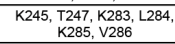 \\
\hline & 3 & 2 & -3.62 & & & & & & & K245(NZ) & & & & & K245(NZ) & & & & 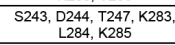 \\
\hline & 4 & 5 & -3.43 & & & & & & & & & & & K245(NZ) & & & $\mathrm{V} 286(\mathrm{O}, \mathrm{N})$ & $\mathrm{K} 245(\mathrm{O})$ & $\begin{array}{l}R 220,024, \text { T247, H } 249 \\
V_{2286}\end{array}$ \\
\hline & 5 & 2 & -3.35 & & & & & & & & & & & K245(NZ) & & & $\begin{array}{lll}L 284(0) \\
\text { V286(N) }\end{array}$ & & S222, D244, K245 \\
\hline & $6 \_1$ & 1 & -3.22 & & & & & & & & & & & & & & & & $\begin{array}{l}\text { D244, K245, T247, L284, } \\
\text { V286 }\end{array}$ \\
\hline \multirow{6}{*}{ frame10 } & 1 1_1 & 16 & -4.34 & & & & & & & & & & & K283(NZ) & & D244(OD2) & & & 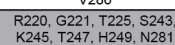 \\
\hline & $2 \_1$ & 14 & -4.30 & & & & & & & K245(NZ) & & & & K283(NZ) & & & & & 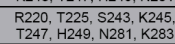 \\
\hline & $3-1$ & 3 & -3.43 & & & & & & & & K245(NZ) & & & K283(NZ) & & $\begin{array}{l}\mathrm{D} 244(\mathrm{OD} 2), \\
\mathrm{K} 245(\mathrm{NZ})\end{array}$ & & $\mathrm{D} 244(0)$ & 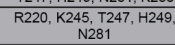 \\
\hline & $4-1$ & 5 & -3.38 & & & & & & & K283(NZ) & K283(NZ) & & & & $\mathrm{K} 245(\mathrm{NZ})$ & & & & 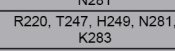 \\
\hline & $5-1$ & 4 & -2.78 & & & & & & & K245(NZ) & & & & & & $\begin{array}{l}\text { D244(OD2), } \\
\text { K245(NZ) }\end{array}$ & & & 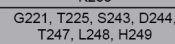 \\
\hline & $6 \_1$ & 1 & -2.69 & & $\begin{array}{c}\text { K245 } \\
\text { (NZ) }\end{array}$ & $\begin{array}{l}\text { K245 } \\
(\text { (ZZ) }\end{array}$ & & & & & & & K245(N) & & & 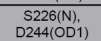 & & & S226, K245, T247 \\
\hline \multirow{6}{*}{ frame15 } & $1-1$ & 3 & -4.45 & & & & & & & K283(NZ) & K283(NZ) & & R220(NH2) & G221(N) & R220(NH2) & & & & 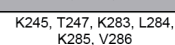 \\
\hline & $2 \_1$ & 7 & -4.39 & & & & & & & K283(NZ) & & & & K245(NZ) & & & & & 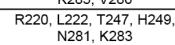 \\
\hline & $3-1$ & 9 & -4.15 & & & & & & & & $\begin{array}{lll}2221(N) \\
\text { a.222(N) }\end{array}$ & & & K283(NZ) & K283(NZ) & & & & K245, Т247, К2283, L284 \\
\hline & $4-1$ & 4 & -3.64 & & $\begin{array}{l}\text { T247 } \\
(0 G 1)\end{array}$ & & & & & & K283(NZ) & & & & $\mathrm{K} 245(\mathrm{NZ})$ & & & $\mathrm{D} 244(0)$ & 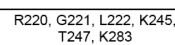 \\
\hline & $5 \_1$ & 1 & -3.60 & & & & & & & & & & & & V286(N) & & & K245(O) & 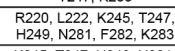 \\
\hline & $6 \_1$ & 3 & -3.60 & & & & & & & & & & & K245(NZ) & & & K245(O) & & 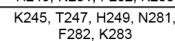 \\
\hline \multirow{6}{*}{ frame20 } & $1-1$ & 3 & -3.20 & & & & & & & & R220(NE) & & & & K283(NZ) & & T247(0G1) & & 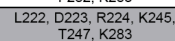 \\
\hline & $2 \_1$ & 8 & -3.20 & & & & & & K245(NZ) & K245(NZ) & & & & K283(NZ) & K283(NZ) & & & & 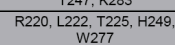 \\
\hline & $3-1$ & 18 & -3.18 & & $\begin{array}{l}\text { R220 } \\
(\mathrm{NEE})\end{array}$ & & & & & & K245(NZ) & & & & & & & & R220, L222, W277 \\
\hline & $4-1$ & 3 & -3.09 & & & & & & & D223(N) & & & & & K245(NZ) & & $\begin{array}{l}\mathrm{D} 223(0) \\
\mathrm{R} 224(0)\end{array}$ & & 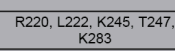 \\
\hline & $5-1$ & 5 & -3.06 & & & & & & & K283(NZ) & & & & $\frac{R_{2}^{220(N H 1)}}{\left.\mathrm{NH}_{2}\right)}$ & & & & & L2222, D244, K245, T247 \\
\hline & $6 \_1$ & 6 & -2.99 & & & & & & & & R220(NE) & & & & K283(NZ) & & T247(OG1) & & $\begin{array}{l}\text { L2222, D223, R224, K245, } \\
\text { T247, K283 }\end{array}$ \\
\hline \multirow{6}{*}{ frame25 } & $1-1$ & 17 & -5.22 & & & & & & & & & & & & & & & & 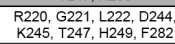 \\
\hline & $2 \_1$ & 2 & -4.56 & & $\begin{array}{l}\text { T247 } \\
(0 \mathrm{G} 1)\end{array}$ & $\begin{array}{l}\mathrm{H} 249 \\
\text { (NE2) }\end{array}$ & & & & & & & & & K283(NZ) & & K283(NZ) & $\mathrm{D} 244(0)$ & 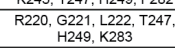 \\
\hline & $3-1$ & 3 & -3.78 & & $\begin{array}{l}T 247 \\
(011)\end{array}$ & $\begin{array}{l}\text { H249 } \\
(\text { NE2) }\end{array}$ & & & & K283(NZ) & K283(NZ) & & & & & & & & R2220, G221, L222, H249 \\
\hline & $4{ }_{-} 1$ & 2 & -3.74 & $\begin{array}{l}{ }_{\text {H249 }}{ }_{\text {(NE2) }} \\
\end{array}$ & & & & & & & K283(NZ) & & & & & & & & 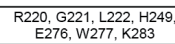 \\
\hline & $55_{-1}$ & 7 & -3.60 & & & & & & & $\begin{array}{ll}\text { K283(NZ) } \\
\end{array}$ & & & & & & K283(NZ) & & & 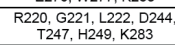 \\
\hline & $66_{-1}$ & 5 & -3.60 & & & & & & & & & & & H249(NE2) & T247(OG1) & & K283(NZ) & & 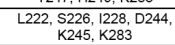 \\
\hline & $1-1$ & 1 & -5.16 & & & $\begin{array}{l}\begin{array}{l}\mathrm{R} 220 \\
(\mathrm{NH} 2)\end{array} \\
\end{array}$ & & & & K245(NZ) & & & & K283(NZ) & & & & 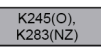 & 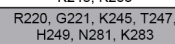 \\
\hline & $2-1$ & 3 & -3.67 & & & & & & & & & & & & & K245(NZ) & & K283(NZ) & 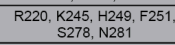 \\
\hline frame30 & 3 & 3 & -4.30 & & & & & & & & K283(NZ) & & & K245(NZ) & & & & & 1228, K245, W246 \\
\hline andineso & $4-1$ & 4 & -4.19 & & & & & & & K245(NZ) & & & & & & K245(NZ) & & K283(NZ) & R220, T225, K245 \\
\hline & $5-1$ & 1 & -4.09 & & & & & & & & K283(NZ) & & & & & & & & D244, K245, W246 \\
\hline & $6-1$ & 11 & -3.93 & & & & & & & K283(NZ) & K283(NZ) & & & & & & & & 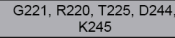 \\
\hline
\end{tabular}


Table 8.5: All 96-well screening conditions that led to crystals of PaAtg18.

\begin{tabular}{|c|c|c|c|c|c|c|c|c|c|c|}
\hline \multirow[t]{2}{*}{ Project } & \multirow{2}{*}{$\begin{array}{c}\text { Protein } \\
\text { conc. } \\
(\mathrm{mg} / \mathrm{mL})\end{array}$} & \multicolumn{2}{|c|}{ Precipitant 1} & \multicolumn{2}{|c|}{ Precipitant 2} & \multicolumn{2}{|l|}{ Buffer } & \multicolumn{2}{|l|}{ Salt } & \multirow[t]{2}{*}{ Crystal characterization } \\
\hline & & Chemical & Conc. & Chemical & Conc. & Buffer & $\mathrm{pH}$ & Chemical & Conc. & \\
\hline paAtg18 & $10 / 20 / 35$ & $\begin{array}{l}\text { Ammonium citrate } \\
\text { dibasic }\end{array}$ & $1 \mathrm{M}$ & & & $\begin{array}{l}0.1 \mathrm{M} \mathrm{Na} \text {-acetate } \\
\text { trihydrate }\end{array}$ & 4.60 & & & multiple needles \\
\hline paAtg18-microseeding & $10 / 30$ & PEG6000 & $20 \% \mathrm{w} / \mathrm{v}$ & & & $\begin{array}{l}0.1 \mathrm{M} \text { Citric acid } \\
\text { anhydrous }\end{array}$ & 4.00 & Lichloride & $1 \mathrm{M}$ & needles \\
\hline paAtg18-microseeding & $10 / 30$ & PEG300 & $40 \% v / v$ & & & $\begin{array}{c}0.1 \mathrm{M} \mathrm{Na} \text { cacodylate } \\
\text { trihydrate }\end{array}$ & 6.50 & $\begin{array}{c}\text { Ca acetate } \\
\text { hydrate }\end{array}$ & $0.2 \mathrm{M}$ & needles + spherulites \\
\hline paAtg18-microseeding & $10 / 30$ & PEG8000 & $14.4 \% \mathrm{w} / \mathrm{v}$ & $\begin{array}{c}\text { glycerol } \\
\text { anhydrous }\end{array}$ & $20 \% \mathrm{w} / \mathrm{v}$ & $\begin{array}{c}0.1 \mathrm{M} \mathrm{Na} \text { cacodylate } \\
\text { trihydrate }\end{array}$ & 6.50 & $\begin{array}{c}\text { Ca acetate } \\
\text { hydrate }\end{array}$ & $0.2 \mathrm{M}$ & needles + spherulites \\
\hline paAtg18-microseeding & $10 / 30$ & $\begin{array}{c}\text { pentaerythritol } \\
\text { ethoxylate }(17 / 8 \\
\text { EO/OH) }\end{array}$ & $40 \% \mathrm{w} / \mathrm{v}$ & & & $0.1 \mathrm{M}$ MES & 5.50 & $\mathrm{MgCl}$ & $0.2 \mathrm{M}$ & needles \\
\hline paAtg18-microseeding & $10 / 30$ & $\begin{array}{c}\text { pentaerythritol } \\
\text { propoxylate }(5 / 4 \\
\mathrm{PO} / \mathrm{OH}) \\
\end{array}$ & $50 \% v / v$ & & & $0.1 \mathrm{M}$ Tris & 8.00 & & & needles \\
\hline paAtg18-microseeding & $10 / 30$ & $\begin{array}{c}\text { pentaerythritol } \\
\text { ethoxylate (15/4 } \\
\mathrm{EO} / \mathrm{OH})\end{array}$ & $15 \% \mathrm{v} / \mathrm{v}$ & Jeffamine T-403 & $3 \% \mathrm{v} / \mathrm{v}$ & $0.1 \mathrm{M}$ MES & 6.00 & $\mathrm{~K}$ acetate & $0.2 \mathrm{M}$ & needles \\
\hline paAtg18-microseeding & $10 / 30$ & $\begin{array}{c}\text { poly(acrylic acid sodium } \\
\text { salt) } 2100\end{array}$ & $30 \% \mathrm{w} / \mathrm{v}$ & & & 0.1 HEPES & 7.00 & Na malonate & $0.1 \mathrm{M}$ & needles \\
\hline paAtg18-microseeding & $10 / 30$ & $\begin{array}{c}\text { polyvinylpyrrolidone } \mathrm{K} \\
15 \\
\end{array}$ & $15 \% \mathrm{w} / \mathrm{v}$ & PEG MME 5000 & $25 \% \mathrm{w} / \mathrm{v}$ & $0.1 \mathrm{M}$ Tris & 8.00 & & & needles \\
\hline paAtg18-microseeding & $10 / 30$ & PEG 3350 & $20 \% \mathrm{w} / \mathrm{v}$ & & & & & Ca chloride & $0.2 \mathrm{M}$ & birefringent crystals + spherulites \\
\hline paAtg18-microseeding & $10 / 30$ & PEG 3350 & $20 \% \mathrm{w} / \mathrm{v}$ & & & & & $\begin{array}{l}\text { Ca acetate } \\
\text { hydrate }\end{array}$ & $0.2 \mathrm{M}$ & needles + spherulites \\
\hline paAtg18-microseeding & $10 / 30$ & PEG 4000 & $30 \% \mathrm{w} / \mathrm{v}$ & & & $0.1 \mathrm{M}$ HEPES & 7.50 & $\begin{array}{l}\text { Ca chloride } \\
\text { dihydrate }\end{array}$ & $0.2 \mathrm{M}$ & needles \\
\hline paAtg18-microseeding & $10 / 30$ & PEG 4000 & $30 \% \mathrm{w} / \mathrm{v}$ & & & $0.1 \mathrm{M}$ Tris & 8.50 & $\begin{array}{c}\text { Li sulfate } \\
\text { monohydrate }\end{array}$ & $0.2 \mathrm{M}$ & needles \\
\hline paAtg18-microseeding & $10 / 30$ & PEG 4000 & $30 \% \mathrm{w} / \mathrm{v}$ & & & $0.1 \mathrm{M}$ Tris & 8.50 & $\begin{array}{c}\mathrm{MgCl} \\
\text { hexahydrate }\end{array}$ & $0.2 \mathrm{M}$ & needles \\
\hline paAtg18-microseeding & $10 / 30$ & & & & & $0.1 \mathrm{M} \mathrm{MES}$ & 6.50 & $\begin{array}{c}\text { Li sulfate } \\
\text { monohydrate }\end{array}$ & $1 \mathrm{M}$ & needles \\
\hline paAtg18-microseeding & $10 / 30$ & ammonium sulfate & $1.26 \mathrm{M}$ & & & $\begin{array}{l}0.1 \mathrm{M} \mathrm{Na} \text {-acetate } \\
\text { trihydrate }\end{array}$ & 4.50 & $\mathrm{NaCl}$ & $0.2 \mathrm{M}$ & needles \\
\hline paAtg18-microseeding & $10 / 30$ & PEG 3000 & $20 \% \mathrm{w} / \mathrm{v}$ & & & $0.1 \mathrm{M}$ Tris & 7.00 & $\begin{array}{l}\text { Ca acetate } \\
\text { hydrate }\end{array}$ & $0.2 \mathrm{M}$ & needles \\
\hline paAtg18-microseeding & $10 / 30$ & $\begin{array}{l}\text { K Na tartrate } \\
\text { tetrahydrate }\end{array}$ & $1 \mathrm{M}$ & & & $0.1 \mathrm{M}$ Tris & 7.00 & $\begin{array}{c}\text { Li sulfate } \\
\text { monohydrate }\end{array}$ & $0.2 \mathrm{M}$ & needles \\
\hline paAtg18-microseeding & 10 & $\begin{array}{c}\text { (+/-)-2-methyl-2,4- } \\
\text { pentanediol }\end{array}$ & $30 \% \mathrm{v} / \mathrm{v}$ & & & $0.1 \mathrm{M} \mathrm{Na}$-acetate & 4.60 & $\begin{array}{l}\text { Ca chloride } \\
\text { dihydrate }\end{array}$ & $\begin{array}{c}0.02 \\
M\end{array}$ & needles \\
\hline paAtg18-microseeding & $10 / 30$ & ammonium sulfate & $0.8 \mathrm{M}$ & & & $\begin{array}{c}\text { sodium citrate tribasic } \\
\text { dihydrate }\end{array}$ & 4.00 & & & needles \\
\hline paAtg18-microseeding & 10 & PEG400 & $30 \% \mathrm{v} / \mathrm{v}$ & & & $\begin{array}{l}0.1 \mathrm{M} \mathrm{Na} \text {-acetate } \\
\text { trihydrate }\end{array}$ & 4.60 & $\begin{array}{l}\text { cadmium } \\
\text { chloride }\end{array}$ & 0.1 & needles + spherulites \\
\hline
\end{tabular}


Table 8.6: All 96-well screening conditions that led to crystals of PaAtg18. Continuation from Table 8.8

\begin{tabular}{|c|c|c|c|c|c|c|c|c|c|c|}
\hline & & & & & & & & hydrate & & \\
\hline paAtg18-microseeding & $10 / 30$ & $\mathrm{NaCl}$ & $2 \mathrm{M}$ & & & $\begin{array}{c}0.1 \mathrm{M} \mathrm{Na} \text {-acetate } \\
\text { trihydrate }\end{array}$ & 4.60 & & & needles \\
\hline paAtg18-microseeding & 30 & Na acetate trihydrate & $1 \mathrm{M}$ & & & $0.1 \mathrm{M}$ HEPES & 7.50 & $\begin{array}{l}\text { Cadmium } \\
\text { sulphate } \\
\text { hydrate }\end{array}$ & $\begin{array}{c}0.05 \\
M\end{array}$ & spherulite+crystals \\
\hline paAtg18-proteinaseK & $10 / 30$ & PEG 8000 & $20 \% \mathrm{w} / \mathrm{v}$ & $\mathrm{NaCl}$ & $0.2 \mathrm{M}$ & $\begin{array}{l}0.1 \mathrm{M} \text { citric acid } \\
\text { anhydrous }\end{array}$ & 4.20 & $\begin{array}{l}\text { di-sodium } \\
\text { hydrogen } \\
\text { phosphate }\end{array}$ & $0.1 \mathrm{M}$ & plates \\
\hline paAtg18-proteinasek & 30 & & & & & $\begin{array}{l}0.1 \mathrm{M} \mathrm{Na} \text {-acetate } \\
\text { trihydrate }\end{array}$ & 4.60 & $\begin{array}{l}\text { ammonium } \\
\text { phosphate } \\
\text { monobasic }\end{array}$ & $1.8 \mathrm{M}$ & spherulites+crystals \\
\hline paAtg18 & $10 / 20 / 30 / 40$ & $\begin{array}{c}\text { Ammonium citrate } \\
\text { dibasic }\end{array}$ & $1.69 \mathrm{M}$ & & & $\begin{array}{c}0.1 \mathrm{M} \mathrm{Na} \text {-acetate } \\
\text { trihydrate }\end{array}$ & 4.00 & & & needles \\
\hline paAtg18-microseeding & $10 / 30$ & Ammonium sulfate & $0.8 \mathrm{M}$ & & & citric acid anhydrous & 4.00 & & & needles \\
\hline paAtg18-subtilisin & $10 / 30$ & & & & & $\begin{array}{l}0.1 \mathrm{M} \mathrm{Na} \text {-acetate } \\
\text { trihydrate }\end{array}$ & 4.60 & $\begin{array}{l}\text { ammonium } \\
\text { phosphate } \\
\text { monobasic }\end{array}$ & $1.8 \mathrm{M}$ & spherulites+crystals \\
\hline paAtg18-subtilisin & $10 / 30$ & $\begin{array}{l}\text { Na phosphate } \\
\text { monobasic } \\
\text { monohydrate }\end{array}$ & $0.8 \mathrm{M}$ & $\begin{array}{l}\text { K phosphate } \\
\text { monobasic }\end{array}$ & $0.8 \mathrm{M}$ & $0.1 \mathrm{M}$ HEPES sodium & 7.50 & & & multiple plates \\
\hline paAtg18-microseeding & $10 / 30$ & & & & & $0.1 \mathrm{M}$ MES & 5.74 & $\begin{array}{c}\text { Li sulfate } \\
\text { monohydrate }\end{array}$ & $\begin{array}{c}0.99 \\
M\end{array}$ & needles \\
\hline paAtg18-microseeding & 10 & & & & & $0.1 \mathrm{M} \mathrm{MES}$ & 6.29 & $\begin{array}{c}\text { Li sulfate } \\
\text { monohydrate }\end{array}$ & $\begin{array}{c}0.99 \\
M\end{array}$ & needles \\
\hline paAtg18-microseeding & $10 / 30$ & & & & & $0.1 \mathrm{M}$ MES & 6.83 & $\begin{array}{c}\text { Li sulfate } \\
\text { monohydrate }\end{array}$ & $\begin{array}{c}0.99 \\
M\end{array}$ & needles \\
\hline paAtg18-microseeding & 30 & & & & & $0.1 \mathrm{M}$ MES & 6.83 & $\begin{array}{c}\text { Li sulfate } \\
\text { monohydrate }\end{array}$ & $\begin{array}{c}1.15 \\
M\end{array}$ & needles \\
\hline paAtg18-microseeding & 10 & & & & & $0.1 \mathrm{M}$ MES & 7.10 & $\begin{array}{c}\text { Li sulfate } \\
\text { monohydrate }\end{array}$ & $\begin{array}{c}0.99 \\
M\end{array}$ & needles \\
\hline paAtg18-microseeding & 30 & ammonium sulfate & $1.26 \mathrm{M}$ & & & $\begin{array}{c}0.1 \mathrm{M} \mathrm{Na} \text {-acetate } \\
\text { trihydrate }\end{array}$ & 4.50 & $\mathrm{NaCl}$ & $0.2 \mathrm{M}$ & needles \\
\hline paAtg18-microseeding & 30 & PEG8000 & $20 \%$ & & & $\begin{array}{c}0.1 \mathrm{M} \text { MES } \\
\text { monohydrate }\end{array}$ & 6.00 & $\begin{array}{c}\text { Ca acetate } \\
\text { hydrate }\end{array}$ & $0.2 \mathrm{M}$ & plates \\
\hline paAtg18-microseeding & $10 / 30$ & & & & & $\begin{array}{l}0.1 \mathrm{M} \mathrm{Na} \text {-acetate } \\
\text { trihydrate }\end{array}$ & 4.60 & Na fluoride & $0.6 \mathrm{M}$ & needles that appear and disappear \\
\hline paAtg18-microseeding & $10 / 30$ & K phosphate monobasic & $0.45 \mathrm{M}$ & $\begin{array}{c}\text { Na phosphate } \\
\text { monobasic }\end{array}$ & $0.45 \mathrm{M}$ & $\begin{array}{c}0.1 \mathrm{M} \text { MES } \\
\text { monohydrate }\end{array}$ & 6.50 & & & needles that appear and disappear \\
\hline paAtg18-microseeding & $10 / 30$ & Na fluoride & $0.6 \mathrm{M}$ & & & $\begin{array}{c}0.1 \mathrm{M} \text { Tris } \\
\text { monohydrate }\end{array}$ & 8.50 & & & \\
\hline paAtg18-microseeding & $10 / 30$ & Na succinate & $0.5 \mathrm{M}$ & & & $\begin{array}{l}0.1 \mathrm{M} \mathrm{Na} \text {-acetate } \\
\text { trihydrate }\end{array}$ & 4.60 & & & needles that appear and disappear \\
\hline paAtg18-proteinaseK & $10 / 30$ & PEG8000 & $20 \%$ & & & $\begin{array}{l}0.1 \mathrm{M} \text { phosphate- } \\
\text { citrate }\end{array}$ & 4.20 & Na chloride & $0.2 \mathrm{M}$ & plates \\
\hline paAtg18-proteinaseK & $10 / 30$ & PEG3350 & $20 \%$ & & & & & $\begin{array}{c}\text { ammonium } \\
\text { citrate } \\
\text { dibasic } \\
\end{array}$ & $0.2 \mathrm{M}$ & multiple plates \\
\hline
\end{tabular}


Table 8.7: All 96-well screening conditions that led to crystals of PaAtg18. Continuation from Table 8.8

\begin{tabular}{|c|c|c|c|c|c|c|c|c|c|c|}
\hline paAtg18-proteinaseK & 30 & $\begin{array}{l}\text { PEG6000 } \\
\end{array}$ & $20 \%$ & & & $\begin{array}{c}0.1 \mathrm{M} \mathrm{Na} \text { acetate } \\
\text { trihydrate }\end{array}$ & 5.00 & Li chloride & $0.2 \mathrm{M}$ & irregular plates \\
\hline paAtg18-proteinaseK & $10 / 30$ & K phosphate dibasic & $0.14 \mathrm{M}$ & & & & & $\begin{array}{c}\mathrm{Na} \\
\text { phosphate } \\
\text { dibasic }\end{array}$ & $\begin{array}{c}1.26 \\
M\end{array}$ & multiple plates \\
\hline paAtg18-proteinaseK & $10 / 30$ & $\begin{array}{c}\text { Ammonium phosphate } \\
\text { monobasic }\end{array}$ & $2 \mathrm{M}$ & & & 0.1 Tris $\mathrm{HCl}$ & 8.50 & & & multiple needles \\
\hline $\begin{array}{c}\text { paAtg18- } \\
\text { proteinaseK+microseed }\end{array}$ & 30 & $\begin{array}{l}\text { Na phosphate } \\
\text { monobasic } \\
\text { monohydrate }\end{array}$ & $0.8 \mathrm{M}$ & $\begin{array}{l}\text { K phosphate } \\
\text { monobasic }\end{array}$ & $0.8 \mathrm{M}$ & $0.1 \mathrm{M}$ HEPES sodium & 7.50 & & & multiple needles \\
\hline paAtg18-proteinasek & $10 / 30$ & $\begin{array}{l}\text { Na phosphate } \\
\text { monobasic } \\
\text { monohydrate }\end{array}$ & $0.8 \mathrm{M}$ & $\begin{array}{l}\text { K phosphate } \\
\text { monobasic }\end{array}$ & $0.8 \mathrm{M}$ & 0.1 M HEPES sodium & 7.50 & & & multiple needles \\
\hline paAtg18-microseeding & $10 / 30$ & $\begin{array}{l}\text { Na phosphate } \\
\text { monobasic } \\
\text { monohydrate }\end{array}$ & $0.45 \mathrm{M}$ & $\begin{array}{l}\text { K phosphate } \\
\text { monobasic }\end{array}$ & $0.45 \mathrm{M}$ & $0.1 \mathrm{M}$ HEPES sodium & 7.50 & & & needles \\
\hline paAtg18-microseeding & $10 / 30$ & Na succinate & $0.5 \mathrm{M}$ & & & $0.1 \mathrm{M}$ HEPES & 7.50 & & & needles \\
\hline paAtg18-microseeding & $10 / 30$ & $\begin{array}{l}\text { Na phosphate } \\
\text { monobasic } \\
\text { monohydrate }\end{array}$ & $0.45 \mathrm{M}$ & $\begin{array}{l}\text { K phosphate } \\
\text { monobasic }\end{array}$ & $0.45 \mathrm{M}$ & $0.1 \mathrm{M}$ Tris $\mathrm{HCl}$ & 8.50 & & & needles \\
\hline paAtg18-microseeding & $10 / 30$ & $\begin{array}{l}\text { K Na tartrate } \\
\text { tetrahydrate }\end{array}$ & $0.6 \mathrm{M}$ & & & $0.1 \mathrm{M} \mathrm{Na}$ Tartrate & 5.60 & & & disappearing needles \\
\hline paAtg18-microseeding & $10 / 30$ & Na sulfate decahydrate & $0.75 \mathrm{M}$ & & & $\begin{array}{c}0.1 \mathrm{M} \text { MES } \\
\text { monohydrate }\end{array}$ & 6.50 & & & needles \\
\hline paAtg18-microseeding & $10 / 30$ & $\begin{array}{l}\text { K Na tartrate } \\
\text { tetrahydrate }\end{array}$ & $0.6 \mathrm{M}$ & & & $\begin{array}{c}0.1 \mathrm{M} \text { MES } \\
\text { monohydrate }\end{array}$ & 6.50 & & & needles \\
\hline paAtg18-microseeding & $10 / 30$ & $\begin{array}{l}\text { K Na tartrate } \\
\text { tetrahydrate }\end{array}$ & $0.6 \mathrm{M}$ & & & $0.1 \mathrm{M} \mathrm{HEPES}$ & 7.50 & & & disappearing needles \\
\hline paAtg18-microseeding & $10 / 30$ & Na sulfate decahydrate & $0.75 \mathrm{M}$ & & & $\begin{array}{c}0.1 \mathrm{M} \text { Tris } \\
\text { hydrochloride }\end{array}$ & 8.50 & & & disappearing needles \\
\hline paAtg18-microseeding & $10 / 30$ & $\begin{array}{l}\text { K Na tartrate } \\
\text { tetrahydrate }\end{array}$ & $0.6 \mathrm{M}$ & & & $\begin{array}{c}0.1 \mathrm{M} \text { Tris } \\
\text { hydrochloride }\end{array}$ & 8.50 & & & disappearing needles \\
\hline paAtg18-proteinasek & $10 / 30$ & Na fluoride & $0.6 \mathrm{M}$ & & & $\begin{array}{c}0.1 \mathrm{M} \mathrm{Na} \text { acetate } \\
\text { trihydrate }\end{array}$ & 4.60 & & & rods \\
\hline paAtg18-proteinaseK & $10 / 30$ & K phosphate monobasic & $0.45 \mathrm{M}$ & $\begin{array}{c}\text { Na phosphate } \\
\text { monobasic }\end{array}$ & $0.45 \mathrm{M}$ & $\begin{array}{c}0.1 \mathrm{M} \mathrm{Na} \text { acetate } \\
\text { trihydrate }\end{array}$ & 4.60 & & & multiple plates \\
\hline paAtg18-proteinaseK & $10 / 30$ & K phosphate monobasic & $0.45 \mathrm{M}$ & $\begin{array}{c}\text { Na phosphate } \\
\text { monobasic }\end{array}$ & $0.45 \mathrm{M}$ & $\begin{array}{c}0.1 \mathrm{M} \text { MES } \\
\text { monohydrate }\end{array}$ & 6.50 & & & multiple plates \\
\hline paAtg18-proteinaseK & 30 & K phosphate monobasic & $0.45 \mathrm{M}$ & $\begin{array}{c}\text { Na phosphate } \\
\text { monobasic }\end{array}$ & $0.45 \mathrm{M}$ & $\begin{array}{c}0.1 \mathrm{M} \text { Tris } \\
\text { hydrochloride }\end{array}$ & 7.00 & & & multiple plates \\
\hline paAtg18-proteinaseK & $10 / 30$ & K phosphate monobasic & $0.45 \mathrm{M}$ & $\begin{array}{c}\text { Na phosphate } \\
\text { monobasic }\end{array}$ & $0.45 \mathrm{M}$ & $\begin{array}{c}0.1 \mathrm{M} \text { MES } \\
\text { monohydrate }\end{array}$ & 6.50 & & & multiple plates \\
\hline paAtg18-proteinaseK & 30 & K phosphate monobasic & $0.9 \mathrm{M}$ & $\begin{array}{c}\text { Na phosphate } \\
\text { monobasic }\end{array}$ & $0.9 \mathrm{M}$ & $\begin{array}{c}0.1 \mathrm{M} \text { MES } \\
\text { monohydrate }\end{array}$ & 6.50 & & & multiple plates \\
\hline paAtg18-proteinaseK & $10 / 30$ & K phosphate monobasic & $0.9 \mathrm{M}$ & $\begin{array}{c}\text { Na phosphate } \\
\text { monobasic }\end{array}$ & $0.9 \mathrm{M}$ & $0.1 \mathrm{M}$ HEPES & 7.50 & & & multiple plates \\
\hline paAtg18-proteinaseK & $10 / 30$ & K phosphate monobasic & $0.9 \mathrm{M}$ & $\begin{array}{c}\text { Na phosphate } \\
\text { monobasic }\end{array}$ & $0.9 \mathrm{M}$ & $0.1 \mathrm{M}$ HEPES & 7.50 & & & multiple plates \\
\hline
\end{tabular}


Table 8.8: All 96-well screening conditions that led to crystals of PaAtg18. Continuation from Table 8.8

\begin{tabular}{|c|c|c|c|c|c|c|c|c|c|c|}
\hline $\begin{array}{c}\text { paAtg18- } \\
\text { proteinaseK+microseed }\end{array}$ & 30 & Na malonate & $1.2 \mathrm{M}$ & & & $\begin{array}{c}0.1 \mathrm{M} \text { MES } \\
\text { monohydrate }\end{array}$ & 6.50 & & & multiple plates \\
\hline $\begin{array}{c}\text { paAtg18- } \\
\text { proteinaseK+microseed }\end{array}$ & 30 & K phosphate monobasic & $0.9 \mathrm{M}$ & $\begin{array}{c}\text { Na phosphate } \\
\text { monobasic }\end{array}$ & $0.9 \mathrm{M}$ & $\begin{array}{c}0.1 \mathrm{M} \mathrm{Na} \text { acetate } \\
\text { trihydrate }\end{array}$ & 4.60 & & & multiple plates \\
\hline $\begin{array}{c}\text { paAtg18- } \\
\text { proteinaseK+microseed }\end{array}$ & $10 / 30$ & K phosphate monobasic & $0.9 \mathrm{M}$ & $\begin{array}{c}\text { Na phosphate } \\
\text { monobasic }\end{array}$ & $0.9 \mathrm{M}$ & $\begin{array}{c}0.1 \mathrm{M} \text { MES } \\
\text { monohydrate }\end{array}$ & 6.50 & & & multiple plates \\
\hline $\begin{array}{c}\text { paAtg18- } \\
\text { proteinaseK+microseed }\end{array}$ & 30 & K phosphate monobasic & $0.9 \mathrm{M}$ & $\begin{array}{c}\text { Na phosphate } \\
\text { monobasic }\end{array}$ & $0.9 \mathrm{M}$ & $0.1 \mathrm{M} \mathrm{HEPES}$ & 7.50 & & & multiple plates \\
\hline $\begin{array}{c}\text { paAtg18- } \\
\text { proteinaseK+microseed }\end{array}$ & $10 / 30$ & K phosphate monobasic & $0.9 \mathrm{M}$ & $\begin{array}{c}\text { Na phosphate } \\
\text { monobasic }\end{array}$ & $0.9 \mathrm{M}$ & $\begin{array}{c}0.1 \mathrm{M} \text { Tris } \\
\text { hydrochloride }\end{array}$ & 8.50 & & & multiple plates \\
\hline $\begin{array}{c}\text { paAtg18- } \\
\text { proteinaseK+microseed }\end{array}$ & 30 & $\begin{array}{l}\mathrm{K} \mathrm{Na} \text { tartrate } \\
\text { tetrahydrate }\end{array}$ & $1.2 \mathrm{M}$ & & & $\begin{array}{c}0.1 \mathrm{M} \text { Tris } \\
\text { hydrochloride }\end{array}$ & 8.50 & & & spherulites \\
\hline $\begin{array}{c}\text { paAtg18- } \\
\text { proteinaseK+microseed }\end{array}$ & 30 & Na malonate & $1.2 \mathrm{M}$ & & & $\begin{array}{c}0.1 \mathrm{M} \text { MES } \\
\text { monohydrate }\end{array}$ & 6.50 & & & multiple plates \\
\hline $\begin{array}{c}\text { paAtg18- } \\
\text { proteinaseK+microseed }\end{array}$ & $10 / 30$ & Na malonate & $1.2 \mathrm{M}$ & & & $0.1 \mathrm{M}$ HEPES & 7.50 & & & multiple plates \\
\hline $\begin{array}{c}\text { paAtg18- } \\
\text { proteinaseK+microseed }\end{array}$ & $10 / 30$ & K phosphate monobasic & $0.9 \mathrm{M}$ & $\begin{array}{c}\text { Na phosphate } \\
\text { monobasic }\end{array}$ & $0.9 \mathrm{M}$ & $\begin{array}{c}0.1 \mathrm{M} \mathrm{Na} \text { acetate } \\
\text { trihydrate }\end{array}$ & 4.60 & & & multiple plates \\
\hline $\begin{array}{c}\text { paAtg18- } \\
\text { proteinaseK+microseed }\end{array}$ & $10 / 30$ & $\mathrm{~K}$ phosphate monobasic & $0.9 \mathrm{M}$ & $\begin{array}{c}\text { Na phosphate } \\
\text { monobasic }\end{array}$ & $0.9 \mathrm{M}$ & $\begin{array}{c}0.1 \mathrm{M} \text { MES } \\
\text { monohydrate }\end{array}$ & 6.50 & & & multiple plates \\
\hline $\begin{array}{c}\text { paAtg18- } \\
\text { proteinaseK+microseed }\end{array}$ & $10 / 30$ & K phosphate monobasic & $0.9 \mathrm{M}$ & $\begin{array}{c}\text { Na phosphate } \\
\text { monobasic }\end{array}$ & $0.9 \mathrm{M}$ & $0.1 \mathrm{M}$ HEPES & 7.50 & & & multiple plates \\
\hline $\begin{array}{c}\text { paAtg18- } \\
\text { proteinaseK+microseed }\end{array}$ & $10 / 30$ & K phosphate monobasic & $0.9 \mathrm{M}$ & $\begin{array}{c}\text { Na phosphate } \\
\text { monobasic }\end{array}$ & $0.9 \mathrm{M}$ & $\begin{array}{c}0.1 \mathrm{M} \text { Tris } \\
\text { hydrochloride }\end{array}$ & 8.50 & & & multiple plates \\
\hline paAtg18-proteinaseK & $10 / 30$ & $\mathrm{Na} / \mathrm{K}$ phosphate & $1.8 \mathrm{M}$ & & & & & & & multiple needles \\
\hline paAtg18-proteinaseK & $10 / 30$ & $\mathrm{Na} / \mathrm{K}$ phosphate & $1.8 \mathrm{M}$ & & & & & & & \\
\hline paAtg18-proteinaseK & 30 & PEG8000 & $20 \% \mathrm{w} / \mathrm{v}$ & $\mathrm{NaCl}$ & $0.2 \mathrm{M}$ & citric acid anhydrous & 4.20 & $\begin{array}{l}\text { di-sodium } \\
\text { hydrogen } \\
\text { phoshate }\end{array}$ & $0.1 \mathrm{M}$ & multiple plates \\
\hline paAtg18-proteinaseK & $10 / 30$ & $\begin{array}{l}\text { Na phosphate } \\
\text { monobasic } \\
\text { monohydrate }\end{array}$ & $0.8 \mathrm{M}$ & $\begin{array}{c}\text { K phosphate } \\
\text { monobasic }\end{array}$ & $0.8 \mathrm{M}$ & $0.1 \mathrm{M}$ HEPES sodium & 7.50 & & & multiple plates \\
\hline
\end{tabular}




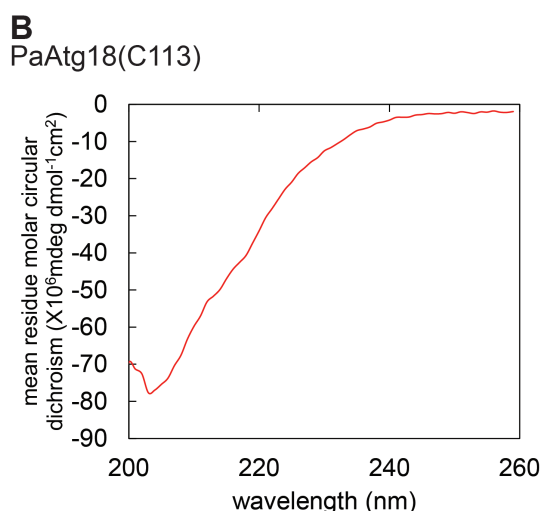

PaAtg18(S157C)

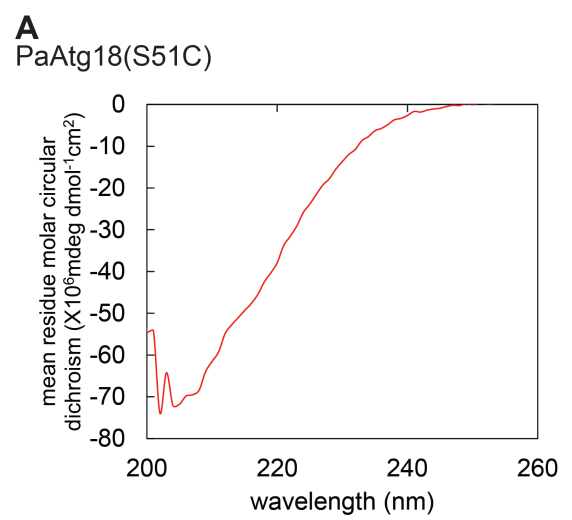

PaAtg18(C113)

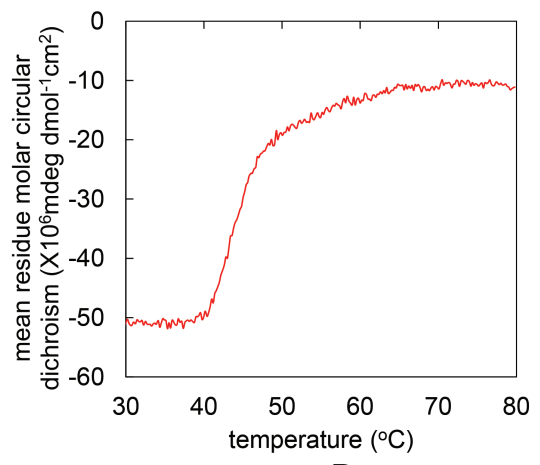

D

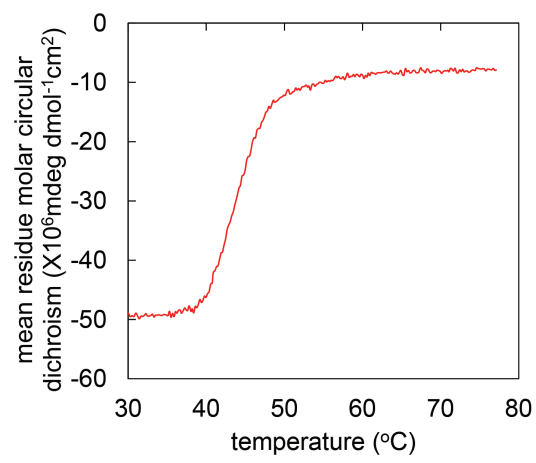

PaAtg18(S459C)
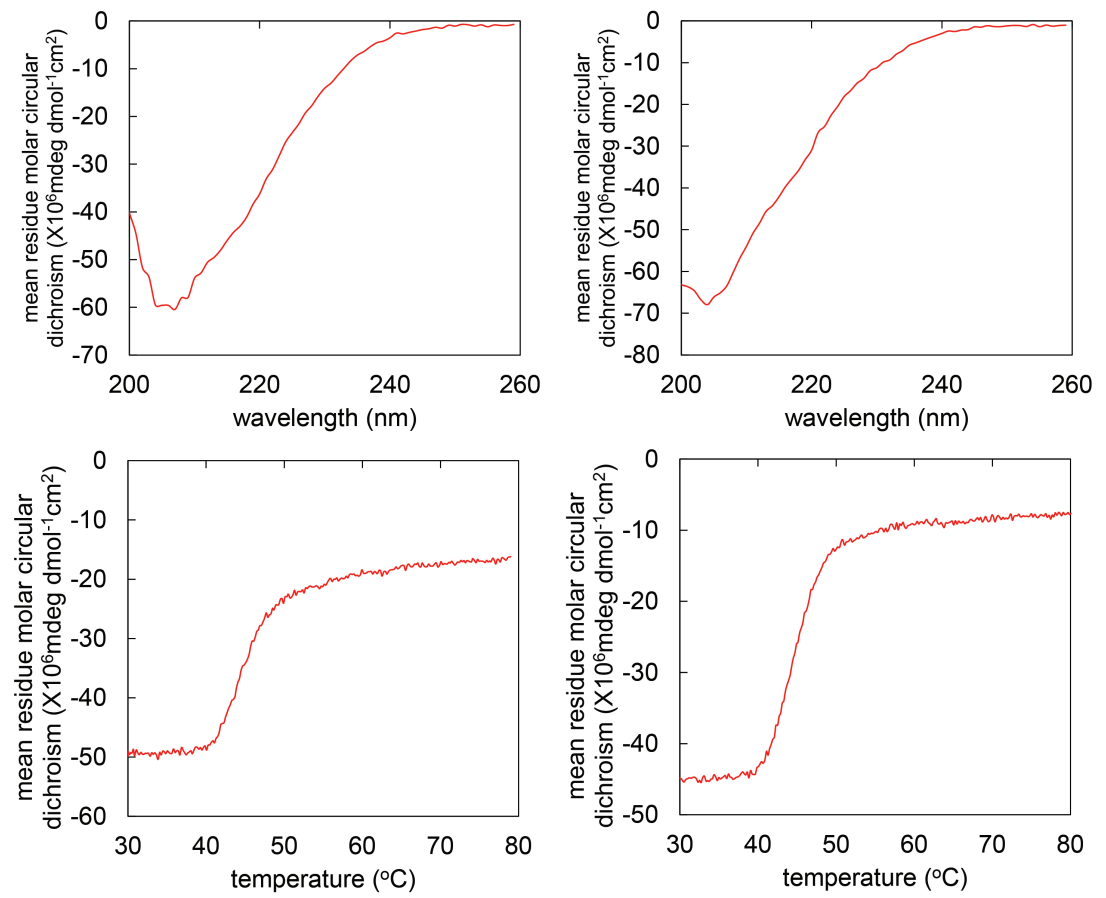

Figure 8.3: Folding controls for PaAtg18 single cysteine mutants. Both the CD spectra from $260 \mathrm{~nm}$ to $200 \mathrm{~nm}$ and melting curves were taken at $216 \mathrm{~nm}$ from $30^{\circ} \mathrm{C}$ to $80^{\circ} \mathrm{C}$. The following protein mutants were investigated: (A) PaAtg18(S51C), (A) PaAtg18(C113), (C) PaAtg18(S157C), (D) PaAtg18(S448C) (D) and PaAtg18(S459C) (E). 


\subsection{DNA sequences of synthetic genes}

\subsubsection{C. thermophilum Atg18}

CATATGGCCGCGACTTTAAACTATGTCACCTTCAACCAAGACCATTCATGCTTGGCGGTGGCGAC TTCTAAGGGATTTCGGATTTTTCATACGGAGCCTTTCGCTAAGGTATTCAACTCAGAAGACGGTC ATGTTTCTATCATAGAAATGCTCTTTAGCACATCACTGGTCGCACTCGTTTTAAGTCCACGTCAC TTAGTCATACAGAACACAAAACGGGGATCAGTTATTTGTGAGTTGACCTTTCCATCTGCGGTATT AGCTGTTCGCCTTAACCGAAAAAGGCTGGCAGTGGTGTTAGAGGAAGAGATCTATCTGTATGAC ATCTCAAACATGGCTCTCGTGAGTACCATTGCTACCTCACCAAACCCGAATGCAATCTTCGCTCT TAGTCCGAGCAGCGAGCGCTGCTACATTGCATACCCGTTACCAAAAGCGCGAGAAGACAACGGC GAAAGAAGACCAGCGCACGCACCACCACTTTCGACCTACGTTCCGCCAACGAGTGGAGAAGTCC TTATCTTTGACGCGCTGACTCTTAAAGCAGTGACTGTGATCGAGGCGCATAAGTCTCCCCTGTGC TGCGTCGCACTTAATTCAGACGGCAACCTTCTGGCGACCGCCAGTGAAACCGGGACGATAATTA GAGTTTTTGCTGTACCATCCGGCCAGAAGTTATATCAGTTTCGTCGTGGTACGTACCCGTCAACC ATATACTCGATGTCTTTTAATCTGAGTAGTACACTGCTGTGCGTAAGTAGTGCCAGCGAGACAGT GCACATTTTTAGATTAGTTACGCCACAGACCGCTTCCAGCGCAGCGTCCAGAGATGCTGATATAC CGTCAAGTCCTAGAGCAAATCGATGGAGTCGCAGTCTGTCCGTAGACTCTACCGATTACCCAGG CTCGTCCGTGGGGGAGGTGGGCGAGCCGTCCCCAAACAATAATGGTACATCCTCTAAACGTTCG TCAGGATCATTCGGATCTTTACTGCGACGTTCCTCACAGCTGGTTGGGCGATCTGTTGCTGGGGC CGTGGGTTCTTATCTGCCACAGTCGGTAACGGAGATGTTCGACCCTCAAAGAGACTTCGCTTCAT TCAAAATACCTCGTCCACATCAAAATGGCGGACGCTCTGGCGCACTGATGGGAGGTTCCTCGGC TCCTCTGCGTTCTGTTGTTGCGATGAGCAGCTCTAGTCCGCAGGTCATGGTAGTGACATCGGATG GCCATTTTTATGTTTACAATATAGATATGCAAAGAGGCGGGGAATGCCCGTTGGTGAAGTTTGTA AGTGTGCTTGATCCGGAAGAAAAACTGGATGCTAGTGCTTATGGAGTGTAATAACTCGAG

\subsubsection{C. thermophilum Atg2}

CTTAAGATGGCGTCCTTTTTCCAGTCCTTTCGTAGCTCAGTAATGCCGAAGCGCTTATTGCGATA CGCGCTCTCCCGCCTCGATTTTTTGGATACCGATGCTCTGGATCTGGAAAATCTCGATTTCGCTCT CGGCAGGAACACTGTTTTGGAATTTCGTGATGTAGGTCTGGTACTTGCGAAACTTGAACGGCTGT TGGGCCTGCCACCGGCGTTCTCACTGCAGAAGGCGAAAGTGCTCATTCTTCGAGTTACCATTCCG ATGGATTTTTACACATCCCCTATTGTTGCGGAGGTTGATGGAGTCGATATCCGTGTGAAAGTTTC CCACAATAAAGCTGAGACGGATGATATTCAGAAAGGCAAAGGCAGCACGGTCGGTGAGGATAT TGTCCCAACGGCTGCAGATCTTGCACAGAGCTTTTTAGAGACCCAACCTCCTGCTGAGAAAAAA CAGCTCGAAGAGGCCATTGCGGCTGAAACTCAAGATTTAGGGGCGAGTATATTATCTGAGTCGG AAAGCAGCGATGATGATTCGACAACCGGGACTGGCCAACCTCTGAGCCTCCCAGTGTTTCTTAC GGACTTCCTCCAAGGCATCGTAGATAGAATGCAGGTGAGAATCGGGACAGTGACCTTTCAGGGC GATGTTGAGGTCCCTGTCGATCCGGATGTAGCTGCGCCGGAGACAGTTACTTTTCAGCTTTCCCT GGAGAGTATTAATGTTGAGGGTGTGACGACCTTACCCGATACTGCAGATGGGAACTCGTGCACT CATGCTGTTGTGCATAAAGATGGGAAACGGCATATACTGCTGAACAATATCCGCGCTGCCCTCA TCTCCGAAGCTAATGTTTTTGCATCATTGGGCCCGTCCCCGAGTATGCATTCAAGCCTTGCATCC 
AAAAGTCCAGTGGCATCTGAGGAACCGCGAGCTCCGAATTTGGCAGAGTCCGTCAGTCGTCGTA GTGGCTATGGATCGTTAGCGGGATCAGCTGCACAGTTGCAACCGTTAAGTAGCTCACGACAGTC TAGTGTGCGGGATTCAGAAGAAGCTCTGGGCATTCCGTATGATTTTGGGGAAGACGAAACGGAT GAACAGGAAGAACAGGGCGGTCCTGCATCTAGCTTATCTACGCCGCGTGCGAGTTTTCATCAGG ATCTCCTGTGCGAGCGCCCGACAGCTCTGTCCAGAGATCCGGCTAAAAGTACCGTCGTGGAACG CTTCAGGGGCGAATGGTCAACGTATGAACGTGAAGCGCGTTCGGAACCAGATCTGCGCCCCCCG GAAGGCTTCAGTTTATCAACTACCCAGTCGCCCCAGGAATCCATACATTCTTCAGGCTCCGCTTC AGCTCAGAGCAGCCGTCGCGCCAGTATCGAAGACCTGACGCAGAGTCAGCTGTATAGCCACGAG GACGCTGAGAGCATGTACATGTCTGCGTTCTCAGAAGTTGGTTCTAGCAAAATGCGTACGGCCA TGCCCGGTGCATGGGAGGATTCGGAGGATTCCCAGCAACAGGACGAGCCGCCAAAAGATGAGG AGTTTGGGACCCGATCTGCCGCGGCCAGCACACCCCCCCTGGAAGGTCCTCCACAGGCACGGGA ACGCGTTGAGTCAGAACCGGCAGAGGAGGGAGAACCCCCGCAGGAGCTGCTTCAGCAGGAAAT CGAACAATTGCCGGAGCTGTCTGCCCAAGAACCAGAACCTGCCCCTCAAGATGACGTTCCGACA CCTCGTGGTCCAACGCGTTTAGCTAAAGAAATTATGCTCTTGGATTCCATCTCTGTCTACCTCCC GACGGCCCACAAACACCTCCAGGTGACAACCCCGGACTTGGCCCGTAGTGTCTCTCCGAATGTG CCTGGCGCGTTTTCGGTTCACTCGGCAGCCGGAAAATCGCAGATTTTACCTATGACGCCGGCCCG GGCAGAACACTCCTCGACCGATGGAGCCATTGAGGTAATCATGAAACCGTTAGATATTCGCTTT GACGCCTCAACTGGATTCCTGCTTGCCAAGGTGATTTCCTCACTGCTGGAAGCCTTACAGCAGTC GAGCGAACCAACCCGGCCGGCGCATTCCTCGAACGATAAGTCGGATGCGACCCCGTCTACCACG TCGCCATTACCTGATCTTCGGATTACCTTAGAGGGTCTGTCTATCCAGTTCCTTGAAAAACTGGC AGGGGTCGCTGATACCCCATCCAGGATCTTTAGTCGTCAC

Because of cost efficiency C. thermophilum Atg2 was ordered in three parts. This is the N-terminal part used as template in the PCR amplification reactions.

\subsubsection{A. thaliana Atg18a}

CCATGGGCAGCAGCCATCATCATCATCATCACAGCAGCGGCCTGGTGCCGCGCGGCAGCCATAT GGCAACTGTAAGCAGCTCCTCCTGGCCCAACCCTAACCCTAATCCAGATTCGACAAGCGCTTCC GATAGTGATAGCACGTTTCCGAGTCATCGTGACCGGGTTGACGAACCGGACTCTTTAGACAGCT TTTCCAGTATGTCATTAAATAGTGATGAACCGAATCAGACTTCAAATCAGTCCCCTCTCTCACCG CCTACTCCGAACTTACCAGTTATGCCACCCCCGAGTGTACTGCATTTATCCTTCAACCAGGACCA TGCGTGCTTTGCCGTTGGAACAGACCGTGGTTTTCGAATTTTAAACTGCGACCCGTTTCGCGAAA TCTTTCGGCGCGATTTTGATAGGGGTGGGGGCGTTGCTGTTGTTGAGATGCTTTTTCGATGCAAC ATACTGGCCCTGGTCGGTGGGGGTCCAGATCCCCAGTATCCGCCTAACAAAGTGATGATATGGG ATGATCATCAGGGTCGTTGCATCGGTGAATTAAGTTTTCGTTCGGATGTGCGGAGTGTACGGCTT CGAAGGGATCGGATTATCGTGGTGCTGGAGCAGAAAATATTTGTGTACAATTTTAGCGACTTAA AACTGATGCATCAAATCGAAACGATCGCGAATCCAAAAGGGTTATGCGCGGTTAGTCAGGGCGT GGGATCAATGGTACTGGTTTGCCCAGGGCTGCAGAAAGGCCAAGTTAGGATAGAGCATTATGCA AGCAAGCGCACCAAGTTCGTTATGGCACATGATTCGCGGATAGCCTGCTTTGCTTTAACGCAGG ATGGTCATCTTCTGGCCACTGCATCCTCAAAAGGCACTTTGGTTCGCATATTCAACACTGTTGAC GGTACTCTTAGACAGGAAGTGAGAAGGGGGGCTGACCGTGCCGAAATCTATTCTCTTGCCTTTTC 
TTCAAATGCTCAATGGCTGGCAGTCAGCAGTGACAAAGGGACCGTGCATGTTTTTGGTTTGAAA GTTAACTCTGGATCTCAGGTCAAAGATTCCAGCCGGATCGCGCCGGATGCAACACCGTCTTCCCC CTCATCATCTCTGAGTCTCTTTAAAGGCGTTCTGCCACGGTATTTCTCCTCAGAATGGAGTGTGG CACAATTCCGGCTGGTGGAAGGCACACAATACATCGCGGCATTTGGACACCAGAAAAACACTGT GGTGATTCTAGGTATGGATGGGTCCTTCTACCGCTGTCAGTTTGATCCGGTCAACGGCGGCGAAA TGTCTCAGCTGGAATATCACAATTGCTTGAAACCACCTTCTGTGTTTTAATAACTCGAG

\subsubsection{A. thaliana WRKY33}

CCATGGGCAGCGCATGGAGTCATCCTCAATTCGAAAAATCCGGACTGGTGCCGCGCGGCAGCCA TATGGCAGCTTCGTTTCTTACAATGGATAATTCTCGAACCCGCCAGAATATGAATGGATCTGCCA ATTGGTCACAGCAGAGTGGACGTACCAGTACTTCATCACTTGAAGATCTGGAAATACCTAAATT TCGTTCCTTCGCACCGTCGTCAATTTCAATCTCGCCCTCGCTGGTGTCTCCGAGCACGTGTTTTAG CCCСTCCCTCTTCCTGGATAGCCCCGCGTTTGTAAGTAGCAGCGCTAACGTGTTAGCCTCCCCCA CCACTGGTGCCCTGATCACGAATGTGACGAATCAAAAGGGAATTAATGAGGGCGATAAATCCAA TAACAACAATTTTAACCTGTTTGACTTCAGCTTTCACACTCAGTCGTCCGGCGTTAGTGCCCCGA CAACGACCACAACCACCACCACTACTACCACAACTACCAATTCGAGTATTTTTCAGTCACAGGA ACAGCAGAAAAAGAATCAATCTGAGCAGTGGAGTCAAACTGAGACAAGACCTAATAACCAGGC TGTATCATATAACGGTCGCGAACAGCGTAAAGGGGAGGATGGATACAACTGGCGGAAATATGG CCAAAAACAAGTGAAGGGCAGCGAGAATCCTCGTTCGTATTATAAATGTACGTTTCCAAACTGT CCTACAAAGAAAAAAGTGGAACGAAGTCTTGAGGGTCAAATAACCGAAATCGTATATAAAGGC AGTCACAATCATCCGAAACCACAGTCCACCCGCAGATCAAGTAGTTCAAGTAGTACGTTTCATA GTGCTGTGTATAACGCTTCACTGGATCATAATCGCCAAGCATCGTCTGACCAACCCAACTCTAAT AATTCTTTCCATCAGTCAGATAGCTTCGGGATGCAGCAGGAAGATAACACTACCTCGGACAGCG TAGGTGATGATGAATTTGAACAGGGGTCATCCATAGTATCTAGGGATGAGGAAGATTGTGGTTC AGAACCGGAGGCGAAACGCTGGAAAGGAGATAATGAAACAAACGGCGGTAATGGTGGCGGCA GTAAAACGGTTCGGGAACCACGGATTGTCGTTCAAACCACGTCTGACATTGACATTCTGGATGA TGGTTACAGATGGCGTAAATACGGACAGAAAGTCGTAAAAGGGAACCCGAATCCGCGCAGCTA TTATAAGTGTACAACCATTGGATGTCCCGTACGAAAGCACGTGGAGCGTGCGTCTCACGATATG CGCGCCGTAATTACAACATACGAAGGGAAACATAACCATGACGTACCGGCGGCGAGAGGCAGT GGCTATGCCACAAACCGCGCCCCACAGGATTCTAGTAGTGTTCCGATTCGGCCCGCAGCCATTG CAGGCCACTCCAATTATACTACCAGCTCTCAGGCCCCATACACACTGCAAATGCTCCATAACAA TAATACCAACACAGGACCCTTTGGATACGCAATGAACAACAACAATAACAATAGCAATCTGCAA ACTCAGCAGAACTTTGTTGGTGGTGGATTTAGCAGAGCCAAGGAGGAACCTAATGAGGAGACTT CATTTTTTGATTCATTTATGCCGTAATAACTCGAG 


\section{Bibliography}

[1] R. Krick, R. A. Busse, A. Scacioc, M. Stephan, A. Janshoff, M. Thumm, and K. Kuhnel. Structural and functional characterization of the two phosphoinositide binding sites of proppins, a beta-propeller protein family. Proc Natl Acad Sci U S A, 109(30):E2042-9, 2012. Krick, Roswitha Busse, Ricarda A Scacioc, Andreea Stephan, Milena Janshoff, Andreas Thumm, Michael Kuhnel, Karin Proc Natl Acad Sci U S A. 2012 Jul 24;109(30):E2042-9. doi: 10.1073/pnas.1205128109. Epub 2012 Jul 2. XIV] 8 , 12, 13, 40, 41, 43, 45, 46, 51, 52, 77, 112, 113, 116, 117, 118, 119

[2] Y. Watanabe, T. Kobayashi, H. Yamamoto, H. Hoshida, R. Akada, F. Inagaki, Y. Ohsumi, and N. N. Noda. Structure-based analyses reveal distinct binding sites for atg2 and phosphoinositides in atg18. Journal of Biological Chemistry, 287(38):31681-31690, 2012. 009XT Times Cited:19 Cited References Count:40. XIV, 8, 9, 12, 13, 16, 18, 101, 112, 118, 119, 120

[3] N. Mizushima, B. Levine, A. M. Cuervo, and D. J. Klionsky. Autophagy fights disease through cellular self-digestion. Nature, 451(7182):1069-75, 2008. Mizushima, Noboru Levine, Beth Cuervo, Ana Maria Klionsky, Daniel J R01 AG021904/AG/NIA NIH HHS/ R01 AG021904-06/AG/NIA NIH HHS/ R03 AG019834/AG/NIA NIH HHS/ R03 AG019834-02/AG/NIA NIH HHS/ R21 AG025355/AG/NIA NIH HHS/ R21 AG025355-02/AG/NIA NIH HHS/ England Nature. 2008 Feb 28;451(7182):1069-75. doi: 10.1038/nature06639. 6

[4] C. M. Kenific and J. Debnath. Cellular and metabolic functions for autophagy in cancer cells. Trends Cell Biol, 2014. Kenific, Candia M Debnath, Jayanta Trends Cell Biol. 2014 Sep 30. pii: S09628924(14)00158-5. doi: 10.1016/j.tcb.2014.09.001. 6

[5] Z. H. Cheung and N. Y. Ip. Autophagy deregulation in neurodegenerative diseases - recent advances and future perspectives. J Neurochem, 118(3):317-25, 2011. Cheung, Zelda H Ip, Nancy Y England J Neurochem. 2011 Aug;118(3):317-25. doi: 10.1111/j.1471-4159.2011.07314.x. Epub 2011 Jun 17. 6

[6] F. Reggiori and D. J. Klionsky. Autophagic processes in yeast: mechanism, machinery and regulation. Genetics, 194(2):341-61, 2013. Reggiori, Fulvio Klionsky, Daniel J R01 GM053396/GM/NIGMS NIH HHS/ Genetics. 2013 Jun;194(2):341-61. doi: 10.1534/genetics.112.149013. 6, 7

[7] A. M. Cuervo and E. Wong. Chaperone-mediated autophagy: roles in disease and aging. Cell Res, 24(1):92-104, 2014. Cuervo, Ana Maria Wong, Esther P01 AG031782/AG/NIA NIH HHS/ R01 AG021904/AG/NIA NIH HHS/ R01 DK098408/DK/NIDDK NIH HHS/ R37 AG021904/AG/NIA NIH HHS/ England Cell Res. 2014 Jan;24(1):92-104. doi: 10.1038/cr.2013.153. Epub 2013 Nov 26. 6 
[8] K. Takeshige, M. Baba, S. Tsuboi, T. Noda, and Y. Ohsumi. Autophagy in yeast demonstrated with proteinase-deficient mutants and conditions for its induction. J Cell Biol, 119(2):301-11, 1992. Takeshige, K Baba, M Tsuboi, S Noda, T Ohsumi, Y J Cell Biol. 1992 Oct;119(2):301-11. 6

[9] T. M. Harding, K. A. Morano, S. V. Scott, and D. J. Klionsky. Isolation and characterization of yeast mutants in the cytoplasm to vacuole protein targeting pathway. J Cell Biol, 131(3):591-602, 1995. Harding, T M Morano, K A Scott, S V Klionsky, D J DK43684/DK/NIDDK NIH HHS/ J Cell Biol. 1995 Nov;131(3):591-602. 7

[10] M. Tsukada and Y. Ohsumi. Isolation and characterization of autophagy-defective mutants of saccharomyces cerevisiae. FEBS Lett, 333(1-2):169-74, 1993. Tsukada, M Ohsumi, Y NETHERLANDS FEBS Lett. 1993 Oct 25;333(1-2):169-74. 7

[11] M. Thumm, R. Egner, B. Koch, M. Schlumpberger, M. Straub, M. Veenhuis, and D. H. Wolf. Isolation of autophagocytosis mutants of saccharomyces cerevisiae. FEBS Lett, 349(2):275-80, 1994. Thumm, M Egner, R Koch, B Schlumpberger, M Straub, M Veenhuis, M Wolf, D H NETHERLANDS FEBS Lett. 1994 Aug 1;349(2):275-80. 7

[12] W. Yuan, P. E. Stromhaug, and Jr. Dunn, W. A. Glucose-induced autophagy of peroxisomes in pichia pastoris requires a unique e1-like protein. Mol Biol Cell, 10(5):1353-66, 1999. Yuan, W Stromhaug, P E Dunn, W A Jr AM-33326/AM/NIADDK NIH HHS/ Mol Biol Cell. 1999 May;10(5):1353-66. 7

[13] Y. Sakai, A. Koller, L. K. Rangell, G. A. Keller, and S. Subramani. Peroxisome degradation by microautophagy in pichia pastoris: identification of specific steps and morphological intermediates. J Cell Biol, 141(3):625-36, 1998. Sakai, Y Koller, A Rangell, L K Keller, G A Subramani, S DK41737/DK/NIDDK NIH HHS/ J Cell Biol. 1998 May 4;141(3):625-36. 7

[14] V. I. Titorenko, I. Keizer, W. Harder, and M. Veenhuis. Isolation and characterization of mutants impaired in the selective degradation of peroxisomes in the yeast hansenula polymorpha. J Bacteriol, 177(2):35763, 1995. Titorenko, V I Keizer, I Harder, W Veenhuis, M J Bacteriol. 1995 Jan;177(2):357-63. 7

[15] H. Mukaiyama, M. Oku, M. Baba, T. Samizo, A. T. Hammond, B. S. Glick, N. Kato, and Y. Sakai. Paz2 and 13 other paz gene products regulate vacuolar engulfment of peroxisomes during micropexophagy. Genes Cells, 7(1):75-90, 2002. Mukaiyama, Hiroyuki Oku, Masahide Baba, Misuzu Samizo, Takeshi Hammond, Adam T Glick, Benjamin S Kato, Nobuo Sakai, Yasuyoshi GM61156/GM/NIGMS NIH HHS/ England Genes Cells. 2002 Jan;7(1):75-90. 7

[16] T. Y. Nazarko, K. Ozeki, A. Till, G. Ramakrishnan, P. Lotfi, M. Yan, and S. Subramani. Peroxisomal atg37 binds atg30 or palmitoyl-coa to regulate phagophore formation during pexophagy. J Cell Biol, 204(4):541-57, 2014. Nazarko, Taras Y Ozeki, Katharine Till, Andreas Ramakrishnan, Geetha Lotfi, Pouya Yan, Mingda Subramani, Suresh DK094843/DK/NIDDK NIH HHS/ GM069373/GM/NIGMS NIH HHS/ GM085764/GM/NIGMS NIH HHS/ K01 DK094843/DK/NIDDK NIH HHS/ J Cell Biol. 2014 Feb 17;204(4):541-57. doi: 10.1083/jcb.201307050. 7 
[17] H. Nakatogawa, K. Suzuki, Y. Kamada, and Y. Ohsumi. Dynamics and diversity in autophagy mechanisms: lessons from yeast. Nat Rev Mol Cell Biol, 10(7):458-67, 2009. Nakatogawa, Hitoshi Suzuki, Kuninori Kamada, Yoshiaki Ohsumi, Yoshinori England Nat Rev Mol Cell Biol. 2009 Jul;10(7):458-67. doi: 10.1038/nrm2708. Epub 2009 Jun 3. 7, 9

[18] K. Suzuki and Y. Ohsumi. Current knowledge of the pre-autophagosomal structure (pas). FEBS Lett, 584(7):1280-6, 2010. Suzuki, Kuninori Ohsumi, Yoshinori Netherlands FEBS Lett. 2010 Apr 2;584(7):1280-6. doi: 10.1016/j.febslet.2010.02.001. Epub 2010 Feb 5. 7

[19] N. N. Noda, Y. Ohsumi, and F. Inagaki. Atg systems from the protein structural point of view. Chem Rev, 109(4):1587-98, 2009. Noda, Nobuo N Ohsumi, Yoshinori Inagaki, Fuyuhiko Chem Rev. 2009 Apr;109(4):1587-98. doi: 10.1021/cr800459r. 8, 9

[20] J. H. Hurley and B. A. Schulman. Atomistic autophagy: The structures of cellular self-digestion. Cell, 157(2), 2014. Ae9dk Times Cited:3 Cited References Count:100. 8

[21] T. Kawamata, Y. Kamada, Y. Kabeya, T. Sekito, and Y. Ohsumi. Organization of the pre-autophagosomal structure responsible for autophagosome formation. Mol Biol Cell, 19(5):2039-50, 2008. Kawamata, Tomoko Kamada, Yoshiaki Kabeya, Yukiko Sekito, Takayuki Ohsumi, Yoshinori Mol Biol Cell. 2008 May;19(5):2039-50. doi: 10.1091/mbc.E07-10-1048. Epub 2008 Feb 20. 8

[22] T. Sekito, T. Kawamata, R. Ichikawa, K. Suzuki, and Y. Ohsumi. Atg17 recruits atg9 to organize the pre-autophagosomal structure. Genes to Cells, 14(5):525-538, 2009. 440OB Times Cited:44 Cited References Count:39. 8

[23] M. J. Ragusa, R. E. Stanley, and J. H. Hurley. Architecture of the atg17 complex as a scaffold for autophagosome biogenesis. Cell, 151(7):1501-1512, 2012. 062AA Times Cited:35 Cited References Count:50. 8

[24] C. C. Jao, M. J. Ragusa, R. E. Stanley, and J. H. Hurley. A horma domain in atg13 mediates pi 3-kinase recruitment in autophagy. Proc Natl Acad Sci U S A, 110(14):5486-5491, 2013. 131ZP Times Cited:12 Cited References Count:34. 8

[25] L. H. Chew, D. Setiaputra, D. J. Klionsky, and C. K. Yip. Structural characterization of the saccharomyces cerevisiae autophagy regulatory complex atg17-atg31-atg29. Autophagy, 9(10):1467-1474, 2013. 260EY Times Cited:3 Cited References Count:28. 8

[26] Y. Fujioka, S. W. Suzuki, H. Yamamoto, C. Kondo-Kakuta, Y. Kimura, H. Hirano, R. Akada, F. Inagaki, Y. Ohsumi, and N. N. Noda. Structural basis of starvation-induced assembly of the autophagy initiation complex. Nature Structural Molecular Biology, 21(6):513-521, 2014. Ai6ts Times Cited:1 Cited References Count:28. 8

[27] G. Stjepanovic, C. W. Davies, R. E. Stanley, M. J. Ragusa, D. J. Kim, and J. H. Hurley. Assembly and dynamics of the autophagy-initiating atg1 complex. Proc Natl Acad Sci U S A, 111(35):12793-12798, 2014. Ao3ln Times Cited:0 Cited References Count:48. 8 
[28] X. H. Li, L. Q. He, K. H. Che, S. F. Funderburk, L. F. Pan, N. N. Pan, M. J. Zhang, Z. Y. Yue, and Y. X. Zhao. Imperfect interface of beclin1 coiled-coil domain regulates homodimer and heterodimer formation with atg141 and uvrag. Nature Communications, 3, 2012. 915XE Times Cited:27 Cited References Count:41. 8

[29] N. N. Noda, T. Kobayashi, W. Adachi, Y. Fujioka, Y. Ohsumi, and F. Inagaki. Structure of the novel c-terminal domain of vacuolar protein sorting 30/autophagy-related protein 6 and its specific role in autophagy. Journal of Biological Chemistry, 287(20):16256-16266, 2012. 942GB Times Cited:5 Cited References Count:49. 8

[30] S. Baskaran, M. J. Ragusa, E. Boura, and J. H. Hurley. Two-site recognition of phosphatidylinositol 3-phosphate by proppins in autophagy. Mol Cell, 47(3):339-348, 2012. 988IT Times Cited:24 Cited References Count:45. 8, 9, 12, 13, 18, 113, 114, 116, 119, 121

[31] F. Reggiori, K. A. Tucker, P. E. Stromhaug, and D. J. Klionsky. The atg1-atg13 complex regulates atg9 and atg23 retrieval transport from the pre-autophagosomal structure. Dev Cell, 6(1):79-90, 2004. 834WX Times Cited:216 Cited References Count:38. 8, 11, 12, 14

[32] C. Behrends, M. E. Sowa, S. P. Gygi, and J. W. Harper. Network organization of the human autophagy system. Nature, 466(7302):68-76, 2010. Behrends, Christian Sowa, Mathew E Gygi, Steven P Harper, J Wade R01 AG011085/AG/NIA NIH HHS/ R01 AG01108518/AG/NIA NIH HHS/ R01 GM054137/GM/NIGMS NIH HHS/ R01 GM054137-14/GM/NIGMS NIH HHS/ R01 GM054137-14S1/GM/NIGMS NIH HHS/ R01 GM054137-15/GM/NIGMS NIH HHS/ R01 GM070565/GM/NIGMS NIH HHS/ R01 GM070565-05S1/GM/NIGMS NIH HHS/ R01 GM095567/GM/NIGMS NIH HHS/ England Nature. 2010 Jul 1;466(7302):68-76. doi: 10.1038/nature09204. Epub 2010 Jun 20. 9, 14, 16, 63, 101

[33] H. Nakatogawa, Y. Ichimura, and Y. Ohsumi. Atg8, a ubiquitin-like protein required for autophagosome formation, mediates membrane tethering and hemifusion. Cell, 130(1):165-78, 2007. Nakatogawa, Hitoshi Ichimura, Yoshinobu Ohsumi, Yoshinori Cell. 2007 Jul 13;130(1):165-78. 9

[34] H. Weidberg, T. Shpilka, E. Shvets, A. Abada, F. Shimron, and Z. Elazar. Lc3 and gate-16 n termini mediate membrane fusion processes required for autophagosome biogenesis. Dev Cell, 20(4):444-54, 2011. Weidberg, Hilla Shpilka, Tomer Shvets, Elena Abada, Adi Shimron, Frida Elazar, Zvulun Dev Cell. 2011 Apr 19;20(4):444-54. doi: 10.1016/j.devcel.2011.02.006. 9

[35] U. Nair, A. Jotwani, J. Geng, N. Gammoh, D. Richerson, W. L. Yen, J. Griffith, S. Nag, K. Wang, T. Moss, M. Baba, J. A. McNew, X. Jiang, F. Reggiori, T. J. Melia, and D. J. Klionsky. Snare proteins are required for macroautophagy. Cell, 146(2):290-302, 2011. Nair, Usha Jotwani, Anjali Geng, Jiefei Gammoh, Noor Richerson, Diana Yen, Wei-Lien Griffith, Janice Nag, Shanta Wang, Ke Moss, Tyler Baba, Misuzu McNew, James A Jiang, Xuejun Reggiori, Fulvio Melia, Thomas J Klionsky, Daniel J CA113890/CA/NCI NIH HHS/ GM071832/GM/NIGMS NIH HHS/ GM53396/GM/NIGMS NIH HHS/ NS063973/NS/NINDS NIH HHS/ R01 GM053396/GM/NIGMS NIH HHS/ R01 GM05339621/GM/NIGMS NIH HHS/ R01 NS063973/NS/NINDS NIH HHS/ Cell. 2011 Jul 22;146(2):290-302. doi: 10.1016/j.cell.2011.06.022. 9 
[36] N. N. Noda, H. Kumeta, H. Nakatogawa, K. Satoo, W. Adachi, J. Ishii, Y. Fujioka, Y. Ohsumi, and F. Inagaki. Structural basis of target recognition by atg8/lc3 during selective autophagy. Genes Cells, 13(12):1211-8, 2008. Noda, Nobuo N Kumeta, Hiroyuki Nakatogawa, Hitoshi Satoo, Kenji Adachi, Wakana Ishii, Junko Fujioka, Yuko Ohsumi, Yoshinori Inagaki, Fuyuhiko England Genes Cells. 2008 Dec;13(12):1211-8. doi: 10.1111/j.1365-2443.2008.01238.x. Epub 2008 Oct 22. 9

[37] K. Satoo, N. N. Noda, H. Kumeta, Y. Fujioka, N. Mizushima, Y. Ohsumi, and F. Inagaki. The structure of atg4b-lc3 complex reveals the mechanism of lc3 processing and delipidation during autophagy. EMBO J, 28(9):1341-50, 2009. Satoo, Kenji Noda, Nobuo N Kumeta, Hiroyuki Fujioka, Yuko Mizushima, Noboru Ohsumi, Yoshinori Inagaki, Fuyuhiko England EMBO J. 2009 May 6;28(9):1341-50. doi: 10.1038/emboj.2009.80. Epub 2009 Mar 26. 9

[38] A. M. Taherbhoy, S. W. Tait, S. E. Kaiser, A. H. Williams, A. Deng, A. Nourse, M. Hammel, I. Kurinov, C. O. Rock, D. R. Green, and B. A. Schulman. Atg8 transfer from atg7 to atg3: a distinctive e1-e2 architecture and mechanism in the autophagy pathway. Mol Cell, 44(3):451-61, 2011. Taherbhoy, Asad M Tait, Stephen W Kaiser, Stephen E Williams, Allison H Deng, Alan Nourse, Amanda Hammel, Michal Kurinov, Igor Rock, Charles O Green, Douglas R Schulman, Brenda A NIH5P30CA021765/CA/NCI NIH HHS/ P30 CA021765/CA/NCI NIH HHS/ P30 CA021765-31/CA/NCI NIH HHS/ P30 CA021765-32/CA/NCI NIH HHS/ P30 CA021765-33/CA/NCI NIH HHS/ R01 AI040646/AI/NIAID NIH HHS/ R01 AI04064608/AI/NIAID NIH HHS/ R01 AI040646-09/AI/NIAID NIH HHS/ R01 AI040646-10/AI/NIAID NIH HHS/ R01 GM034496/GM/NIGMS NIH HHS/ R01 GM077053/GM/NIGMS NIH HHS/ R01 GM077053-03/GM/NIGMS NIH HHS/ R01 GM077053-04/GM/NIGMS NIH HHS/ R01 GM07705305/GM/NIGMS NIH HHS/ R01AI40646/AI/NIAID NIH HHS/ R01GM034496/GM/NIGMS NIH HHS/ R01GM077053/GM/NIGMS NIH HHS/ R37 GM034496/GM/NIGMS NIH HHS/ R37 GM034496-24/GM/NIGMS NIH HHS/ R37 GM034496-25/GM/NIGMS NIH HHS/ R37 GM03449626/GM/NIGMS NIH HHS/ RR-15301/RR/NCRR NIH HHS/ Howard Hughes Medical Institute/ Mol Cell. 2011 Nov 4;44(3):451-61. doi: 10.1016/j.molcel.2011.08.034. 9

[39] N. N. Noda, K. Satoo, Y. Fujioka, H. Kumeta, K. Ogura, H. Nakatogawa, Y. Ohsumi, and F. Inagaki. Structural basis of atg8 activation by a homodimeric e1, atg7. Mol Cell, 44(3):462-75, 2011. Noda, Nobuo N Satoo, Kenji Fujioka, Yuko Kumeta, Hiroyuki Ogura, Kenji Nakatogawa, Hitoshi Ohsumi, Yoshinori Inagaki, Fuyuhiko Mol Cell. 2011 Nov 4;44(3):462-75. doi: 10.1016/j.molcel.2011.08.035. 9

[40] S. B. Hong, B. W. Kim, K. E. Lee, S. W. Kim, H. Jeon, J. Kim, and H. K. Song. Insights into noncanonical e1 enzyme activation from the structure of autophagic e1 atg7 with atg8. Nature Structural Molecular Biology, 18(12):1323-U32, 2011. 861IC Times Cited:18 Cited References Count:50. 9

[41] M. Yamaguchi, K. Matoba, R. Sawada, Y. Fujioka, H. Nakatogawa, H. Yamamoto, Y. Kobashigawa, H. Hoshida, R. Akada, Y. Ohsumi, N. N. Noda, and F. Inagaki. Noncanonical recognition and ubl loading of distinct e2s by autophagy-essential atg7. Nature Structural Molecular Biology, 19(12):12506, 2012. Yamaguchi, Masaya Matoba, Kazuaki Sawada, Ryoko Fujioka, Yuko Nakatogawa, Hitoshi Yamamoto, Hayashi Kobashigawa, Yoshihiro Hoshida, Hisashi Akada, Rinji Ohsumi, Yoshinori Noda, 
Nobuo N Inagaki, Fuyuhiko Nat Struct Mol Biol. 2012 Dec;19(12):1250-6. doi: 10.1038/nsmb.2451. Epub 2012 Nov 11. 9

[42] S. B. Hong, B. W. Kim, J. H. Kim, and H. K. Song. Structure of the autophagic e2 enzyme atg 10. Acta Crystallographica Section D-Biological Crystallography, 68:1409-1417, 2012. 10 008CZ Times Cited:4 Cited References Count:41. 9

[43] S. E. Kaiser, K. Mao, A. M. Taherbhoy, S. Yu, J. L. Olszewski, D. M. Duda, I. Kurinov, A. Deng, T. D. Fenn, D. J. Klionsky, and B. A. Schulman. Noncanonical e2 recruitment by the autophagy e1 revealed by atg7-atg3 and atg7-atg10 structures. Nature Structural Molecular Biology, 19(12):1242-9, 2012. Kaiser, Stephen E Mao, Kai Taherbhoy, Asad M Yu, Shanshan Olszewski, Jennifer L Duda, David M Kurinov, Igor Deng, Alan Fenn, Timothy D Klionsky, Daniel J Schulman, Brenda A 5P30CA021765/CA/NCI NIH HHS/ R01 GM053396/GM/NIGMS NIH HHS/ R01 GM077053/GM/NIGMS NIH HHS/ R01GM053396/GM/NIGMS NIH HHS/ R01GM077053/GM/NIGMS NIH HHS/ RR-15301/RR/NCRR NIH HHS/ Howard Hughes Medical Institute/ Nat Struct Mol Biol. 2012 Dec;19(12):1242-9. doi: 10.1038/nsmb.2415. Epub 2012 Nov 11. 9

[44] M. Yamaguchi, N. N. Noda, H. Yamamoto, T. Shima, H. Kumeta, Y. Kobashigawa, R. Akada, Y. Ohsumi, and F. Inagaki. Structural insights into atg10-mediated formation of the autophagy-essential atg12-atg5 conjugate. Structure, 20(7):1244-1254, 2012. 970TZ Times Cited:14 Cited References Count:47. 9

[45] N. N. Noda, Y. Fujioka, T. Hanada, Y. Ohsumi, and F. Inagaki. Structure of the atg12-atg5 conjugate reveals a platform for stimulating atg8-pe conjugation. EMBO Rep, 14(2):206-11, 2013. Noda, Nobuo N Fujioka, Yuko Hanada, Takao Ohsumi, Yoshinori Inagaki, Fuyuhiko England EMBO Rep. 2013 Feb;14(2):206-11. doi: 10.1038/embor.2012.208. Epub 2012 Dec 14. 9

[46] M. Sakoh-Nakatogawa, K. Matoba, E. Asai, H. Kirisako, J. Ishii, N. N. Noda, F. Inagaki, H. Nakatogawa, and Y. Ohsumi. Atg12-atg5 conjugate enhances e2 activity of atg3 by rearranging its catalytic site. Nature Structural Molecular Biology, 20(4):433-9, 2013. Sakoh-Nakatogawa, Machiko Matoba, Kazuaki Asai, Eri Kirisako, Hiromi Ishii, Junko Noda, Nobuo N Inagaki, Fuyuhiko Nakatogawa, Hitoshi Ohsumi, Yoshinori Nat Struct Mol Biol. 2013 Apr;20(4):433-9. doi: 10.1038/nsmb.2527. Epub 2013 Mar 17. 9

[47] Z. Metlagel, C. Otomo, G. Takaesu, and T. Otomo. Structural basis of atg3 recognition by the autophagic ubiquitin-like protein atg12. Proc Natl Acad Sci U S A, 110(47):18844-9, 2013. Metlagel, Zoltan Otomo, Chinatsu Takaesu, Giichi Otomo, Takanori GM092740/GM/NIGMS NIH HHS/ R01 GM092740/GM/NIGMS NIH HHS/ Howard Hughes Medical Institute/ Proc Natl Acad Sci U S A. 2013 Nov 19;110(47):18844-9. doi: 10.1073/pnas.1314755110. Epub 2013 Nov 4. 9

[48] V. V. Rogov, A. Rozenknop, N. Y. Rogova, F. Lohr, S. Tikole, V. Jaravine, P. Guntert, I. Dikic, and V. Dotsch. A universal expression tag for structural and functional studies of proteins. Chembiochem, 13(7):959-963, 2012. 931CL Times Cited:6 Cited References Count:28. 9

[49] Y. Watanabe, N. N. Noda, H. Kumeta, K. Suzuki, Y. Ohsumi, and F. Inagaki. Selective transport of alpha-mannosidase by autophagic pathways: structural basis for cargo recognition by atg19 and 
atg34. J Biol Chem, 285(39):30026-33, 2010. Watanabe, Yasunori Noda, Nobuo N Kumeta, Hiroyuki Suzuki, Kuninori Ohsumi, Yoshinori Inagaki, Fuyuhiko J Biol Chem. 2010 Sep 24;285(39):30026-33. doi: 10.1074/jbc.M110.143545. Epub 2010 Jul 21. 9

[50] N. Kondo-Okamoto, N. N. Noda, S. W. Suzuki, H. Nakatogawa, I. Takahashi, M. Matsunami, A. Hashimoto, F. Inagaki, Y. Ohsumi, and K. Okamoto. Autophagy-related protein 32 acts as autophagic degron and directly initiates mitophagy. Journal of Biological Chemistry, 287(13):10631-10638, 2012. 917IN Times Cited:20 Cited References Count:40. 9

[51] N. von Muhlinen, M. Akutsu, B. J. Ravenhill, A. Foeglein, S. Bloor, T. J. Rutherford, S. M. V. Freund, D. Komander, and F. Randow. Lc3c, bound selectively by a noncanonical lir motif in ndp52, is required for antibacterial autophagy. Mol Cell, 48(3):329-342, 2012. 039QF Times Cited:44 Cited References Count:57. 9

[52] J. J. Dumas, E. Merithew, E. Sudharshan, D. Rajamani, S. Hayes, D. Lawe, S. Corvera, and D. G. Lambright. Multivalent endosome targeting by homodimeric eea1. Mol Cell, 8(5):947-958, 2001. 494YA Times Cited:139 Cited References Count:39. 9, 10,113

[53] T. Kutateladze and M. Overduin. Structural mechanism of endosome docking by the fyve domain. Science, 291(5509):1793-1796, 2001. 408HK Times Cited:110 Cited References Count:18. 9, 113

[54] K. M. Ferguson, M. A. Lemmon, J. Schlessinger, and P. B. Sigler. Crystal-structure at 2.2-angstrom resolution of the pleckstrin homology domain from human dynamin. Cell, 79(2):199-209, 1994. Pn630 Times Cited:229 Cited References Count:70. 9

[55] J. Bravo, D. Karathanassis, C. M. Pacold, M. E. Pacold, C. D. Ellson, K. E. Anderson, P. J. G. Butler, I. Lavenir, O. Perisic, P. T. Hawkins, L. Stephens, and R. L. Williams. The crystal structure of the px domain from p40(phox) bound to phosphatidylinositol 3-phosphate. Mol Cell, 8(4):829-839, 2001. 487ZE Times Cited:165 Cited References Count:55. 9

[56] H. Hiroaki, T. Ago, T. Ito, H. Sumimoto, and D. Kohda. Solution structure of the px domain, a target of the sh3 domain. Nature Structural Biology, 8(6):526-530, 2001. 436EV Times Cited:124 Cited References Count:26. 9

[57] R. B. Sutton, B. A. Davletov, A. M. Berghuis, T. C. Sudhof, and S. R. Sprang. Structure of the first c2 domain of synaptotagmin i: a novel ca2+/phospholipid-binding fold. Cell, 80(6):929-38, 1995. Sutton, R B Davletov, B A Berghuis, A M Sudhof, T C Sprang, S R Cell. 1995 Mar 24;80(6):929-38. 9

[58] M. M. Zhou, K. S. Ravichandran, E. T. Olejniczak, A. M. Petros, R. P. Meadows, M. Sattler, J. E. Harlan, W. S. Wade, S. J. Burakoff, and S. W. Fesik. Structure and ligand recognition of the phosphotyrosine binding domain of shc. Nature, 378(6557):584-592, 1995. Tj221 Times Cited:295 Cited References Count:50. 9

[59] C. S. Wood, K. R. Schmitz, N. J. Bessman, T. G. Setty, K. M. Ferguson, and C. G. Burd. Ptdins4p recognition by vps74/golph3 links ptdins 4-kinase signaling to retrograde golgi trafficking. Journal of Cell Biology, 187(7):967-975, 2009. 537FS Times Cited:56 Cited References Count:55. 9 
[60] M. G. J. Ford, B. M. F. Pearse, M. K. Higgins, Y. Vallis, D. J. Owen, A. Gibson, C. R. Hopkins, P. R. Evans, and H. T. McMahon. Simultaneous binding of ptdins(4,5)p-2 and clathrin by ap180 in the nucleation of clathrin lattices on membranes. Science, 291(5506):1051-1055, 2001. 400EW Times Cited:439 Cited References Count:36. 9

[61] J. Hyman, H. Chen, P. P. Di Fiore, P. De Camilli, and A. T. Brunger. Epsin 1 undergoes nucleocytosolic shuttling and its eps 15 interactor nh2-terminal homology (enth) domain, structurally similar to armadillo and heat repeats, interacts with the transcription factor promyelocytic leukemia $\mathrm{zn} 2+$ finger protein (plzf). Journal of Cell Biology, 149(3):537-546, 2000. 310MJ Times Cited:122 Cited References Count:58. 9

[62] M. A. Pearson, D. Reczek, A. Bretscher, and P. A. Karplus. Structure of the erm protein moesin reveals the ferm domain fold masked by an extended actin binding tail domain. Cell, 101(3):259-270, 2000. 308ZC Times Cited:361 Cited References Count:74. 9

[63] O. Kocher, G. Birrane, K. Tsukamoto, S. Fenske, A. Yesilaltay, R. Pal, K. Daniels, J. A. A. Ladias, and M. Krieger. In vitro and in vivo analysis of the binding of the $\mathrm{c}$ terminus of the hdl receptor scavenger receptor class b, type i (sr-bi), to the pdz1 domain of its adaptor protein pdzk1. Journal of Biological Chemistry, 285(45):34999-35010, 2010. 673KL Times Cited:6 Cited References Count:48. 9

[64] S. Santagata, T. J. Boggon, C. L. Baird, C. A. Gomez, J. Zhao, W. S. Shan, D. G. Myszka, and L. Shapiro. G-protein signaling through tubby proteins. Science, 292(5524):2041-2050, 2001. 442KP Times Cited:171 Cited References Count:58. 9

[65] J. He, R. M. Haney, M. Vora, V. V. Verkhusha, R. V. Stahelin, and T. G. Kutateladze. Molecular mechanism of membrane targeting by the grp1 ph domain. Journal of Lipid Research, 49(8):1807-1815, 2008. 325CS Times Cited:22 Cited References Count:42. 10, 113

[66] S. A. Lee, R. Eyeson, M. L. Cheever, J. M. Geng, V. V. Verkhusha, C. Burd, M. Overduin, and T. G. Kutateladze. Targeting of the fyve domain to endosomal membranes is regulated by a histidine switch. Proc Natl Acad Sci U S A, 102(37):13052-13057, 2005. 964YG Times Cited:46 Cited References Count:44. 10, 113

[67] N. Verdaguer, S. Corbalan-Garcia, W. F. Ochoa, I. Fita, and J. C. Gomez-Fernandez. Ca2+ bridges the c2 membrane-binding domain of protein kinase c alpha directly to phosphatidylserine. Embo Journal, 18(22):6329-6338, 1999. 258ZT Times Cited:208 Cited References Count:50. 10

[68] C. Zhao, G. W. Du, K. Skowronek, M. A. Frohman, and D. Bar-Sagi. Phospholipase d2-generated phosphatidic acid couples egfr stimulation to ras activation by sos. Nature Cell Biology, 9(6):706-U171, 2007. 174DY Times Cited:140 Cited References Count:33. 10

[69] D. Karathanassis, R. V. Stahelin, J. Bravo, O. Perisic, C. M. Pacold, W. W. Cho, and R. L. Williams. Binding of the px domain of p47(phox) to phosphatidylinositol 3,4-bisphosphate and phosphatidic acid is masked by an intramolecular interaction. Embo Journal, 21(19):5057-5068, 2002. 602JR Times Cited:190 Cited References Count:47. 10, 13 
[70] C. L. Lai, K. E. Landgraf, G. A. Voth, and J. J. Falke. Membrane docking geometry and target lipid stoichiometry of membrane-bound pkcalpha c2 domain: a combined molecular dynamics and experimental study. J Mol Biol, 402(2):301-10, 2010. Lai, Chun-Liang Landgraf, Kyle E Voth, Gregory A Falke, Joseph J R01 GM063235/GM/NIGMS NIH HHS/ R01 GM063235-10/GM/NIGMS NIH HHS/ R01 GM063796/GM/NIGMS NIH HHS/ R01 GM06379611/GM/NIGMS NIH HHS/ R01-GM063235/GM/NIGMS NIH HHS/ R01-GM063796/GM/NIGMS NIH HHS/ T32-GM065103/GM/NIGMS NIH HHS/ England J Mol Biol. 2010 Sep 17;402(2):301-10. doi: 10.1016/j.jmb.2010.07.037. Epub 2010 Jul 24. 10

[71] D. E. Klein, A. Lee, D. W. Frank, M. S. Marks, and M. A. Lemmon. The pleckstrin homology domains of dynamin isoforms require oligomerization for high affinity phosphoinositide binding. J Biol Chem, 273(42):27725-33, 1998. Klein, D E Lee, A Frank, D W Marks, M S Lemmon, M A 1R21AI4261701/AI/NIAID NIH HHS/ J Biol Chem. 1998 Oct 16;273(42):27725-33. 10, 113

[72] A. Godi, A. Di Campli, A. Konstantakopoulos, G. Di Tullio, D. R. Alessi, G. S. Kular, T. Daniele, P. Marra, J. M. Lucocq, and M. A. De Matteis. Fapps control golgi-to-cell-surface membrane traffic by binding to arf and ptdins(4)p. Nature Cell Biology, 6(5):393-404, 2004. Godi, Anna Di Campli, Antonella Konstantakopoulos, Athanasios Di Tullio, Giuseppe Alessi, Dario R Kular, Gursant S Daniele, Tiziana Marra, Pierfrancesco Lucocq, John M De Matteis, M Antonietta GP0203Y01/Telethon/Italy England Nat Cell Biol. 2004 May;6(5):393-404. Epub 2004 Apr 25. 10

[73] C. N. Lumb and M. S. Sansom. Finding a needle in a haystack: the role of electrostatics in target lipid recognition by ph domains. PLoS Comput Biol, 8(7):e1002617, 2012. Lumb, Craig N Sansom, Mark S P Biotechnology and Biological Sciences Research Council/United Kingdom Medical Research Council/United Kingdom Wellcome Trust/United Kingdom PLoS Comput Biol. 2012;8(7):e1002617. doi: 10.1371/journal.pcbi.1002617. Epub 2012 Jul 26. 10, 114

[74] D. S. Brandt, M. D. Coffman, J. J. Falke, and J. D. Knight. Hydrophobic contributions to the membrane docking of synaptotagmin $7 \mathrm{c} 2 \mathrm{a}$ domain: Mechanistic contrast between isoforms 1 and 7 . Biochemistry, 51(39):7654-7664, 2012. 013QQ Times Cited:3 Cited References Count:70. 11, 114

[75] S. A. Lee, J. Kovacs, R. V. Stahelin, M. L. Cheever, M. Overduin, T. G. Setty, C. G. Burd, W. Cho, and T. G. Kutateladze. Molecular mechanism of membrane docking by the vam7p px domain. $J$ Biol Chem, 281(48):37091-101, 2006. Lee, Stephanie A Kovacs, James Stahelin, Robert V Cheever, Matthew L Overduin, Michael Setty, Thanuja Gangi Burd, Christopher G Cho, Wonhwa Kutateladze, Tatiana G BBS/B/10714/Biotechnology and Biological Sciences Research Council/United Kingdom CA95144/CA/NCI NIH HHS/ GM071424/GM/NIGMS NIH HHS/ GM68849/GM/NIGMS NIH HHS/ R01 GM061221/GM/NIGMS NIH HHS/ J Biol Chem. 2006 Dec 1;281(48):37091-101. Epub 2006 Sep 19. 11,114

[76] T. G. Kutateladze, D. G. Capelluto, C. G. Ferguson, M. L. Cheever, A. G. Kutateladze, G. D. Prestwich, and M. Overduin. Multivalent mechanism of membrane insertion by the fyve domain. J Biol Chem, 279 (4):3050-7, 2004. Kutateladze, Tatiana G Capelluto, Daniel G S Ferguson, Colin G Cheever, Matthew L 
Kutateladze, Andrei G Prestwich, Glenn D Overduin, Michael 071684/Wellcome Trust/United Kingdom J Biol Chem. 2004 Jan 23;279(4):3050-7. Epub 2003 Oct 25. 11, 114

[77] M. A. Lemmon. Membrane recognition by phospholipid-binding domains. Nat Rev Mol Cell Biol, 9(2):99-111, 2008. Lemmon, Mark A England Nat Rev Mol Cell Biol. 2008 Feb;9(2):99-111. doi: 10.1038/nrm2328. 11, 117

[78] J. Carlton, M. Bujny, B. J. Peter, V. M. J. Oorschot, A. Rutherford, H. Mellor, J. Klumperman, H. T. McMahon, and P. J. Cullen. Sorting nexin-1 mediates tubular endosome-to-tgn transport through coincidence sensing of high-curvature membranes and 3-phosphoinositides. Current Biology, 14(20):17911800, 2004. 866PA Times Cited:224 Cited References Count:37. 11, 117

[79] K. Obara, T. Sekito, K. Niimi, and Y. Ohsumi. The atg18-atg2 complex is recruited to autophagic membranes via phosphatidylinositol 3-phosphate and exerts an essential function. Journal of Biological Chemistry, 283(35):23972-23980, 2008. 340II Times Cited:84 Cited References Count:33. 11, 14, 15, 61,101

[80] P. O. Seglen and P. B. Gordon. 3-methyladenine: specific inhibitor of autophagic/lysosomal protein degradation in isolated rat hepatocytes. Proc Natl Acad Sci U S A, 79(6):1889-92, 1982. Seglen, P O Gordon, P B Proc Natl Acad Sci U S A. 1982 Mar;79(6):1889-92. 11

[81] E. F. Blommaart, U. Krause, J. P. Schellens, H. Vreeling-Sindelarova, and A. J. Meijer. The phosphatidylinositol 3-kinase inhibitors wortmannin and ly294002 inhibit autophagy in isolated rat hepatocytes. Eur J Biochem, 243(1-2):240-6, 1997. Blommaart, E F Krause, U Schellens, J P VreelingSindelarova, H Meijer, A J GERMANY Eur J Biochem. 1997 Jan 15;243(1-2):240-6. 11

[82] E. F. Blommaart, J. J. Luiken, P. J. Blommaart, G. M. van Woerkom, and A. J. Meijer. Phosphorylation of ribosomal protein s6 is inhibitory for autophagy in isolated rat hepatocytes. J Biol Chem, 270(5): 2320-6, 1995. Blommaart, E F Luiken, J J Blommaart, P J van Woerkom, G M Meijer, A J J Biol Chem. $1995 \mathrm{Feb} 3 ; 270(5): 2320-6.11$

[83] A. Petiot, E. Ogier-Denis, E. F. C. Blommaart, A. J. Meijer, and P. Codogno. Distinct classes of phosphatidylinositol 3 '-kinases are involved in signaling pathways that control macroautophagy in ht-29 cells. Journal of Biological Chemistry, 275(2):992-998, 2000. 275TQ Times Cited:656 Cited References Count:58.

[84] T. Noda and Y. Ohsumi. Tor, a phosphatidylinositol kinase homologue, controls autophagy in yeast. Journal of Biological Chemistry, 273(7):3963-3966, 1998. Yx516 Times Cited:587 Cited References Count:39. 11

[85] D. C. Nice, T. K. Sato, P. E. Stromhaug, S. D. Emr, and D. J. Klionsky. Cooperative binding of the cytoplasm to vacuole targeting pathway proteins, cvt13 and cvt20, to phosphatidylinositol 3-phosphate at the pre-autophagosomal structure is required for selective autophagy. Journal of Biological Chemistry, 277(33):30198-30207, 2002. 585DW Times Cited:99 Cited References Count:45. 11 
[86] S. I. Yamashita, M. Oku, Y. Wasada, Y. Ano, and Y. Sakai. Pi4p-signaling pathway for the synthesis of a nascent membrane structure in selective autophagy. Journal of Cell Biology, 173(5):709-717, 2006. 050AL Times Cited:45 Cited References Count:31.

[87] S. I. Yamashita, M. Oku, and Y. Sakai. Functions of pi4p and sterol glucoside are necessary for the synthesis of a nascent membrane structure during pexophagy. Autophagy, 3(1):35-37, 2007. 126FY Times Cited:7 Cited References Count:16. 11

[88] A. E. Wurmser and S. D. Emr. Novel ptdins(3)p-binding protein etf1 functions as an effector of the vps34 ptdins 3-kinase in autophagy. Journal of Cell Biology, 158(4):761-772, 2002. 587PF Times Cited:64 Cited References Count:60.

[89] P. Isakson, P. Holland, and A. Simonsen. The role of alfy in selective autophagy. Cell Death and Differentiation, 20(1):12-20, 2013. Sp. Iss. SI 051PA Times Cited:4 Cited References Count:89. 11, 13

[90] S. Pankiv, E. A. Alemu, A. Brech, J. A. Bruun, T. Lamark, A. Overvatn, G. Bjorkoy, and T. Johansen. Fyco1 is a rab7 effector that binds to lc 3 and pi3p to mediate microtubule plus end-directed vesicle transport. Journal of Cell Biology, 188(2):253-269, 2010. 547HI Times Cited:94 Cited References Count:62. 11

[91] E. L. Axe, S. A. Walker, M. Manifava, P. Chandra, H. L. Roderick, A. Habermann, G. Griffiths, and N. T. Ktistakis. Autophagosome formation from membrane compartments enriched in phosphatidylinositol 3phosphate and dynamically connected to the endoplasmic reticulum. Journal of Cell Biology, 182(4): 685-701, 2008. 346EA Times Cited:441 Cited References Count:45.

[92] D. Chen, W. Fan, Y. Lu, X. Ding, S. Chen, and Q. Zhong. A mammalian autophagosome maturation mechanism mediated by tecpr1 and the atg12-atg5 conjugate. Mol Cell, 45(5):629-41, 2012. Chen, Dandan Fan, Weiliang Lu, Yiting Ding, Xiaojun Chen, She Zhong, Qing R01 CA133228/CA/NCI NIH HHS/ R01 CA133228-01A1/CA/NCI NIH HHS/ R01 CA133228-02/CA/NCI NIH HHS/ R01 CA13322803/CA/NCI NIH HHS/ R01 CA133228-04/CA/NCI NIH HHS/ R01 CA133228-05/CA/NCI NIH HHS/ Mol Cell. 2012 Mar 9;45(5):629-41. doi: 10.1016/j.molcel.2011.12.036. Epub 2012 Feb 16. 11, 13

[93] P. E. Stromhaug, F. Reggiori, J. Guan, C. W. Wang, and D. J. Klionsky. Atg21 is a phosphoinositide binding protein required for efficient lipidation and localization of atg8 during uptake of aminopeptidase i by selective autophagy. Mol Biol Cell, 15(8):3553-3566, 2004. 841SK Times Cited:106 Cited References Count:36. 12, 61

[94] R. Krick, J. Tolstrup, A. Appelles, S. Henke, and M. Thumm. The relevance of the phosphatidylinositolphosphat-binding motif frrgt of atg18 and atg21 for the cvt pathway and autophagy. FEBS Lett, 580(19):4632-4638, 2006. 077YT Times Cited:37 Cited References Count:33. 12, 45, 61, 65

[95] R. Krick, S. Henke, J. Tolstrup, and M. Thumm. Dissecting the localization and function of atg18, atg21 and ygr223c. Autophagy, 4(7):896-910, 2008. Krick, Roswitha Henke, Sandra Tolstrup, Joern Thumm, Michael Autophagy. 2008 Oct;4(7):896-910. Epub 2008 Oct 18. 12, 61 
[96] H. E. Polson, J. de Lartigue, D. J. Rigden, M. Reedijk, S. Urbe, M. J. Clague, and S. A. Tooze. Mammalian atg18 (wipi2) localizes to omegasome-anchored phagophores and positively regulates lc3 lipidation. Autophagy, 6(4):506-22, 2010. Polson, Hannah E J de Lartigue, Jane Rigden, Daniel J Reedijk, Marco Urbe, Sylvie Clague, Michael J Tooze, Sharon A Cancer Research UK/United Kingdom Wellcome Trust/United Kingdom Autophagy. 2010 May;6(4):506-22. doi: 10.4161/auto.6.4.11863. Epub 2010 May 16. 12, 15, 101

[97] D. Bakula, Z. Takacs, and T. Proikas-Cezanne. Wipi beta-propellers in autophagy-related diseases and longevity. Biochem Soc Trans, 41:962-967, 2013. 4 193LU Times Cited:1 Cited References Count:47. 12

[98] T. B. Haack, P. Hogarth, M. C. Kruer, A. Gregory, T. Wieland, T. Schwarzmayr, E. Graf, L. Sanford, E. Meyer, E. Kara, S. M. Cuno, S. I. Harik, V. H. Dandu, N. Nardocci, G. Zorzi, T. Dunaway, M. Tarnopolsky, S. Skinner, S. Frucht, E. Hanspal, C. Schrander-Stumpel, D. Heron, C. Mignot, B. Garavaglia, K. Bhatia, J. Hardy, T. M. Strom, N. Boddaert, H. H. Houlden, M. A. Kurian, T. Meitinger, H. Prokisch, and S. J. Hayflick. Exome sequencing reveals de novo wdr45 mutations causing a phenotypically distinct, x-linked dominant form of nbia. American Journal of Human Genetics, 91(6): 1144-1149, 2012. 055LI Times Cited:27 Cited References Count:9. 12

[99] H. Saitsu, T. Nishimura, K. Muramatsu, H. Kodera, S. Kumada, K. Sugai, E. Kasai-Yoshida, N. Sawaura, H. Nishida, A. Hoshino, F. Ryujin, S. Yoshioka, K. Nishiyama, Y. Kondo, Y. Tsurusaki, M. Nakashima, N. Miyake, H. Arakawa, M. Kato, N. Mizushima, and N. Matsumoto. De novo mutations in the autophagy gene wdr45 cause static encephalopathy of childhood with neurodegeneration in adulthood. Nature Genetics, 45(4):445-449, 2013. 115VS Times Cited:34 Cited References Count:34. 12

[100] Q. Lu, P. Yang, X. Huang, W. Hu, B. Guo, F. Wu, L. Lin, A. L. Kovacs, L. Yu, and H. Zhang. The wd40 repeat ptdins(3)p-binding protein epg-6 regulates progression of omegasomes to autophagosomes. Dev Cell, 21(2):343-57, 2011. Lu, Qun Yang, Peiguo Huang, Xinxin Hu, Wanqiu Guo, Bin Wu, Fan Lin, Long Kovacs, Attila L Yu, Li Zhang, Hong Dev Cell. 2011 Aug 16;21(2):343-57. doi: 10.1016/j.devcel.2011.06.024. Epub 2011 Jul 28. 12, 15,

[101] Y. Xiong, A. L. Contento, and D. C. Bassham. Atatg18a is required for the formation of autophagosomes during nutrient stress and senescence in arabidopsis thaliana. Plant Journal, 42(4):535-546, 2005. 921EC Times Cited:110 Cited References Count:51. 12, 17, 107

[102] H. Barth, K. Meiling-Wesse, U. D. Epple, and M. Thumm. Autophagy and the cytoplasm to vacuole targeting pathway both require aut10p. FEBS Lett, 508(1):23-28, 2001. 494PD Times Cited:60 Cited References Count:26. 12

[103] J. Guan, P. E. Stromhaug, M. D. George, P. Habibzadegah-Tari, A. Bevan, W. A. Dunn, and D. J. Klionsky. Cvt18/gsa12 is required for cytoplasm-to-vacuole transport, pexophagy, and autophagy in saccharomyces cerevisiae and pichia pastoris. Mol Biol Cell, 12(12):3821-3838, 2001. 501TA Times Cited:97 Cited References Count:60. 12, 61 
[104] S. K. Dove, R. C. Piper, R. K. McEwen, J. W. Yu, M. C. King, D. C. Hughes, J. Thuring, A. B. Holmes, F. T. Cooke, R. H. Michell, P. J. Parker, and M. A. Lemmon. Svp1p defines a family of phosphatidylinositol 3,5-bisphosphate effectors. EMBO J, 23(9):1922-33, 2004. Dove, Stephen K Piper, Robert C McEwen, Robert K Yu, Jong W King, Megan C Hughes, David C Thuring, Jan Holmes, Andrew B Cooke, Frank T Michell, Robert H Parker, Peter J Lemmon, Mark A 5-R01-GM56846/GM/NIGMS NIH HHS/ R01 GM058202/GM/NIGMS NIH HHS/ Wellcome Trust/United Kingdom England EMBO J. 2004 May 5;23(9):1922-33. Epub 2004 Apr 22. 12, 61, 116, 117, 119

[105] J. A. Efe, R. J. Botelho, and S. D. Emr. Atg18 regulates organelle morphology and fab1 kinase activity independent of its membrane recruitment by phosphatidylinositol 3,5-bisphosphate. Mol Biol Cell, 18 (11):4232-44, 2007. Efe, Jem A Botelho, Roberto J Emr, Scott D Mol Biol Cell. 2007 Nov;18(11):423244. Epub 2007 Aug 15. 12,14

[106] N. Tamura, M. Oku, M. Ito, N. N. Noda, F. Inagaki, and Y. Sakai. Atg18 phosphoregulation controls organellar dynamics by modulating its phosphoinositide-binding activity. J Cell Biol, 202(4):685-98, 2013. Tamura, Naoki Oku, Masahide Ito, Moemi Noda, Nobuo N Inagaki, Fuyuhiko Sakai, Yasuyoshi J Cell Biol. 2013 Aug 19;202(4):685-98. doi: 10.1083/jcb.201302067. Epub 2013 Aug 12. 12, 13, 114, 119

[107] U. Nair, Y. Cao, Z. Xie, and D. J. Klionsky. Roles of the lipid-binding motifs of atg 18 and atg21 in the cytoplasm to vacuole targeting pathway and autophagy. J Biol Chem, 285(15):1147688, 2010. Nair, Usha Cao, Yang Xie, Zhiping Klionsky, Daniel J GM53396/GM/NIGMS NIH HHS/ R01 GM053396/GM/NIGMS NIH HHS/ J Biol Chem. 2010 Apr 9;285(15):11476-88. doi: 10.1074/jbc.M109.080374. Epub 2010 Feb 12. 12

[108] N. Tamura, M. Oku, and Y. Sakai. Atg21 regulates pexophagy via its pi(3) p-binding activity in pichia pastoris. Fems Yeast Research, 14(3):435-444, 2014. Ag0qq Times Cited:0 Cited References Count:31. 12

[109] A. N. Leao-Helder, A. M. Krikken, G. Gellissen, I. J. van der Klei, M. Veenhuis, and J. A. K. W. Kiel. Atg21p is essential for macropexophagy and microautophagy in the yeast hansenula polymorpha. FEBS Lett, 577(3):491-495, 2004. 874RU Times Cited:11 Cited References Count:29. 12

[110] K. Meiling-Wesse, H. Barth, C. Voss, E. L. Eskelinen, U. D. Epple, and M. Thumm. Atg21 is required for effective recruitment of atg8 to the preautophagosomal structure during the cvt pathway. Journal of Biological Chemistry, 279(36):37741-37750, 2004. 849QK Times Cited:23 Cited References Count:56. 12

[111] T. Georgakopoulos, G. Koutroubas, I. Vakonakis, M. Tzermia, V. Prokova, A. Voutsina, and D. Alexandraki. Functional analysis of the saccharomyces cerevisiae yfr021w/ygr223c/ypl100w orf family suggests relations to mitochondrial/peroxisomal functions and amino acid signalling pathways. Yeast, 18 (12):1155-1171, 2001. 475JP Times Cited:19 Cited References Count:46. 12, 14

[112] S. Schoebel, W. Blankenfeldt, R. S. Goody, and A. Itzen. High-affinity binding of phosphatidylinositol 4-phosphate by legionella pneumophila drra. EMBO Rep, 11(8):598-604, 2010. Schoebel, Stefan 
Blankenfeldt, Wulf Goody, Roger S Itzen, Aymelt England EMBO Rep. 2010 Aug;11(8):598-604. doi: 10.1038/embor.2010.97. Epub 2010 Jul 9. 13

[113] L. Janda, P. Tichy, J. Spizek, and M. Petricek. A deduced thermomonospora curvata protein containing serine/threonine protein kinase and wd-repeat domains. J Bacteriol, 178(5):1487-9, 1996. Janda, L Tichy, P Spizek, J Petricek, M J Bacteriol. 1996 Mar;178(5):1487-9. 13

[114] M. A. Wall, D. E. Coleman, E. Lee, J. A. Iniguez-Lluhi, B. A. Posner, A. G. Gilman, and S. R. Sprang. The structure of the g protein heterotrimer gi alpha 1 beta 1 gamma 2. Cell, 83(6):104758, 1995. Wall, M A Coleman, D E Lee, E Iniguez-Lluhi, J A Posner, B A Gilman, A G Sprang, S R DK46371/DK/NIDDK NIH HHS/ GM34497/GM/NIGMS NIH HHS/ Cell. 1995 Dec 15;83(6):1047-58. 13

[115] J. Sondek, A. Bohm, D. G. Lambright, H. E. Hamm, and P. B. Sigler. Crystal structure of a g-protein beta gamma dimer at 2.1 angstrom resolution (vol 379, pg 369, 1996). Nature, 379(6568):847-847, 1996. Tx501 Times Cited:13 Cited References Count:1. 13

[116] C. Xu and J. R. Min. Structure and function of wd40 domain proteins. Protein Cell, 2(3):202-214, 2011. 029TV Times Cited:67 Cited References Count:82. 13

[117] W. C. Voegtli, A. Y. Madrona, and D. K. Wilson. The structure of aip1p, a wd repeat protein that regulates cofilin-mediated actin depolymerization. Journal of Biological Chemistry, 278(36):34373-34379, 2003. 716UK Times Cited:44 Cited References Count:36. 13

[118] J. R. R. Whittle and T. U. Schwartz. Structure of the sec13-sec16 edge element, a template for assembly of the copii vesicle coat. Journal of Cell Biology, 190(3):347-361, 2010. 635XK Times Cited:25 Cited References Count:61. 13

[119] C. U. Stirnimann, E. Petsalaki, R. B. Russell, and C. W. Muller. Wd40 proteins propel cellular networks. Trends in Biochemical Sciences, 35(10):565-574, 2010. 670HF Times Cited:87 Cited References Count:69. 13

[120] N. Mizushima, A. Kuma, Y. Kobayashi, A. Yamamoto, M. Matsubae, T. Takao, T. Natsume, Y. Ohsumi, and T. Yoshimori. Mouse apg161, a novel wd-repeat protein, targets to the autophagic isolation membrane with the apg12-apg5 conjugate. Journal of Cell Science, 116(Pt 9):1679-88, 2003. Mizushima, Noboru Kuma, Akiko Kobayashi, Yoshinori Yamamoto, Akitsugu Matsubae, Masami Takao, Toshifumi Natsume, Tohru Ohsumi, Yoshinori Yoshimori, Tamotsu England J Cell Sci. 2003 May 1;116(Pt 9):1679-88. 13

[121] G. M. Fimia, A. Stoykova, A. Romagnoli, L. Giunta, S. Di Bartolomeo, R. Nardacci, M. Corazzari, C. Fuoco, A. Ucar, P. Schwartz, P. Gruss, M. Piacentini, K. Chowdhury, and F. Cecconi. Ambra1 regulates autophagy and development of the nervous system. Nature, 447(7148):1121-5, 2007. Fimia, Gian Maria Stoykova, Anastassia Romagnoli, Alessandra Giunta, Luigi Di Bartolomeo, Sabrina Nardacci, Roberta Corazzari, Marco Fuoco, Claudia Ucar, Ahmet Schwartz, Peter Gruss, Peter Piacentini, Mauro Chowdhury, Kamal Cecconi, Francesco TCR04004/Telethon/Italy England Nature. 2007 Jun 28;447(7148):1121-5. Epub 2007 Jun 24. 13 
[122] J. Ptacek, G. Devgan, G. Michaud, H. Zhu, X. W. Zhu, J. Fasolo, H. Guo, G. Jona, A. Breitkreutz, R. Sopko, R. R. McCartney, M. C. Schmidt, N. Rachidi, S. J. Lee, A. S. Mah, L. Meng, M. J. R. Stark, D. F. Stern, C. De Virgilio, M. Tyers, B. Andrews, M. Gerstein, B. Schweitzer, P. F. Predki, and M. Snyder. Global analysis of protein phosphorylation in yeast. Nature, 438(7068):679-684, 2005. 988JY Times Cited:537 Cited References Count:27. 14

[123] C. C. He, M. Baba, Y. Cao, and D. J. Klionsky. Self-interaction is critical for atg9 transport and function at the phagophore assembly site during autophagy. Mol Biol Cell, 19(12):5506-5516, 2008. 377IK Times Cited:39 Cited References Count:40. 14

[124] N. Pashkova, L. Gakhar, S. C. Winistorfer, L. P. Yu, S. Ramaswamy, and R. C. Piper. Wd40 repeat propellers define a ubiquitin-binding domain that regulates turnover of $\mathrm{f}$ box proteins. Mol Cell, 40(3): 433-443, 2010. 684NJ Times Cited:39 Cited References Count:62. 14

[125] K. Tarassov, V. Messier, C. R. Landry, S. Radinovic, M. M. S. Molina, I. Shames, Y. Malitskaya, J. Vogel, H. Bussey, and S. W. Michnick. An in vivo map of the yeast protein interactome. Science, 320(5882): 1465-1470, 2008. 312MV Times Cited:325 Cited References Count:36. 14

[126] N. Jin, C. Y. Chow, L. Liu, S. N. Zolov, R. Bronson, M. Davisson, J. L. Petersen, Y. L. Zhang, S. Park, J. E. Duex, D. Goldowitz, M. H. Meisler, and L. S. Weisman. Vac14 nucleates a protein complex essential for the acute interconversion of pi3p and pi(3,5)p-2 in yeast and mouse. Embo Journal, 27(24): 3221-3234, 2008. 385AS Times Cited:63 Cited References Count:33. 14

[127] R. Tonikian, X. Xin, C. P. Toret, D. Gfeller, C. Landgraf, S. Panni, S. Paoluzi, L. Castagnoli, B. Currell, S. Seshagiri, H. Yu, B. Winsor, M. Vidal, M. B. Gerstein, G. D. Bader, R. Volkmer, G. Cesareni, D. G. Drubin, P. M. Kim, S. S. Sidhu, and C. Boone. Bayesian modeling of the yeast sh3 domain interactome predicts spatiotemporal dynamics of endocytosis proteins. PLoS Biol, 7(10):e1000218, 2009. Tonikian, Raffi Xin, Xiaofeng Toret, Christopher P Gfeller, David Landgraf, Christiane Panni, Simona Paoluzi, Serena Castagnoli, Luisa Currell, Bridget Seshagiri, Somasekar Yu, Haiyuan Winsor, Barbara Vidal, Marc Gerstein, Mark B Bader, Gary D Volkmer, Rudolf Cesareni, Gianni Drubin, David G Kim, Philip M Sidhu, Sachdev S Boone, Charles GM R01 50399/GM/NIGMS NIH HHS/ MOP-84324/Canadian Institutes of Health Research/Canada PLoS Biol. 2009 Oct;7(10):e1000218. doi: 10.1371/journal.pbio.1000218. Epub 2009 Oct 20. 14

[128] H. Y. Yu, P. Braun, M. A. Yildirim, I. Lemmens, K. Venkatesan, J. Sahalie, T. Hirozane-Kishikawa, F. Gebreab, N. Li, N. Simonis, T. Hao, J. F. Rual, A. Dricot, A. Vazquez, R. R. Murray, C. Simon, L. Tardivo, S. Tam, N. Svrzikapa, C. Y. Fan, A. S. de Smet, A. Motyl, M. E. Hudson, J. Park, X. F. Xin, M. E. Cusick, T. Moore, C. Boone, M. Snyder, F. P. Roth, A. L. Barabasi, J. Tavernier, D. E. Hill, and M. Vidal. High-quality binary protein interaction map of the yeast interactome network. Science, 322 (5898):104-110, 2008. 355AE Times Cited:564 Cited References Count:45. 14

[129] D. L. Swaney, P. Beltrao, L. Starita, A. L. Guo, J. Rush, S. Fields, N. J. Krogan, and J. Villen. Global analysis of phosphorylation and ubiquitylation cross-talk in protein degradation. Nature Methods, 10(7): 676-+, 2013. 172LV Times Cited:16 Cited References Count:47. 14 
[130] T. Ito, T. Chiba, R. Ozawa, M. Yoshida, M. Hattori, and Y. Sakaki. A comprehensive two-hybrid analysis to explore the yeast protein interactome. Proc Natl Acad Sci U S A, 98(8):4569-4574, 2001. 421HV Times Cited:2197 Cited References Count:32. 14

[131] C. S. Vollert and P. Uetz. The phox homology (px) domain protein interaction network in yeast. Molecular Cellular Proteomics, 3(11):1053-1064, 2004. 881CJ Times Cited:26 Cited References Count:51. 14

[132] H. Yu, P. Braun, M. A. Yildirim, I. Lemmens, K. Venkatesan, J. Sahalie, T. Hirozane-Kishikawa, F. Gebreab, N. Li, N. Simonis, T. Hao, J. F. Rual, A. Dricot, A. Vazquez, R. R. Murray, C. Simon, L. Tardivo, S. Tam, N. Svrzikapa, C. Fan, A. S. de Smet, A. Motyl, M. E. Hudson, J. Park, X. Xin, M. E. Cusick, T. Moore, C. Boone, M. Snyder, F. P. Roth, A. L. Barabasi, J. Tavernier, D. E. Hill, and M. Vidal. High-quality binary protein interaction map of the yeast interactome network. Science, 322(5898):104-10, 2008. Yu, Haiyuan Braun, Pascal Yildirim, Muhammed A Lemmens, Irma Venkatesan, Kavitha Sahalie, Julie Hirozane-Kishikawa, Tomoko Gebreab, Fana Li, Na Simonis, Nicolas Hao, Tong Rual, Jean-Francois Dricot, Amelie Vazquez, Alexei Murray, Ryan R Simon, Christophe Tardivo, Leah Tam, Stanley Svrzikapa, Nenad Fan, Changyu de Smet, Anne-Sophie Motyl, Adriana Hudson, Michael E Park, Juyong Xin, Xiaofeng Cusick, Michael E Moore, Troy Boone, Charlie Snyder, Michael Roth, Frederick P Barabasi, Albert-Laszlo Tavernier, Jan Hill, David E Vidal, Marc HG003224/HG/NHGRI NIH HHS/ R01 HG001715/HG/NHGRI NIH HHS/ R01 HG00171506/HG/NHGRI NIH HHS/ R01 HG003224/HG/NHGRI NIH HHS/ R01 HG003224-04/HG/NHGRI NIH HHS/ R01-HG001715/HG/NHGRI NIH HHS/ U01 AI070499-01/AI/NIAID NIH HHS/ U01A1070499-01/PHS HHS/ U56 CA113004/CA/NCI NIH HHS/ U56 CA113004-03/CA/NCI NIH HHS/ U56-CA113004/CA/NCI NIH HHS/ New York, N.Y. Science. 2008 Oct 3;322(5898):104-10. doi: 10.1126/science.1158684. Epub 2008 Aug 21. 14

[133] H. C. Dooley, M. Razi, H. E. J. Polson, S. E. Girardin, M. I. Wilson, and S. A. Tooze. Wipi2 links lc3 conjugation with pi3p, autophagosome formation, and pathogen clearance by recruiting atg12-5-1611. Mol Cell, 55(2):238-252, 2014. An5os Times Cited:1 Cited References Count:45. 14

[134] J. E. Saiz, M. A. Santos, C. R. Vazquez de Aldana, and J. L. Revuelta. Disruption of six unknown open reading frames from saccharomyces cerevisiae reveals two genes involved in vacuolar morphogenesis and one gene required for sporulation. Yeast, 15(2):155-64, 1999. Saiz, J E Santos, M A Vazquez de Aldana, C R Revuelta, J L ENGLAND Chichester, England Yeast. 1999 Jan 30;15(2):155-64. 14

[135] T. Shintani, K. Suzuki, Y. Kamada, T. Noda, and Y. Ohsumi. Apg2p functions in autophagosome formation on the perivacuolar structure. J Biol Chem, 276(32):30452-60, 2001. Shintani, T Suzuki, K Kamada, Y Noda, T Ohsumi, Y J Biol Chem. 2001 Aug 10;276(32):30452-60. Epub 2001 May 29. 14 15, 16, 101

[136] H. Barth and M. Thumm. A genomic screen identifies aut8 as a novel gene essential for autophagy in the yeast saccharomyces cerevisiae. Gene, 274(1-2):151-6. 14, 15, 61, 101 
[137] C. W. Wang, J. Kim, W. P. Huang, H. Abeliovich, P. E. Stromhaug, Jr. Dunn, W. A., and D. J. Klionsky. Apg2 is a novel protein required for the cytoplasm to vacuole targeting, autophagy, and pexophagy pathways. J Biol Chem, 276(32):30442-51, 2001. Wang, C W Kim, J Huang, W P Abeliovich, H Stromhaug, P E Dunn, W A Jr Klionsky, D J GM53396/GM/NIGMS NIH HHS/ R01 GM053396/GM/NIGMS NIH HHS/ R01 GM053396-11/GM/NIGMS NIH HHS/ R01 GM053396-12/GM/NIGMS NIH HHS/ J Biol Chem. 2001 Aug 10;276(32):30442-51. Epub 2001 May 29. 14, 15, 16, 101, 107

[138] K. Suzuki, M. Akioka, C. Kondo-Kakuta, H. Yamamoto, and Y. Ohsumi. Fine mapping of autophagyrelated proteins during autophagosome formation in saccharomyces cerevisiae. Journal of Cell Science, 126(Pt 11):2534-44, 2013. Suzuki, Kuninori Akioka, Manami Kondo-Kakuta, Chika Yamamoto, Hayashi Ohsumi, Yoshinori England J Cell Sci. 2013 Jun 1;126(Pt 11):2534-44. doi: 10.1242/jcs.122960. Epub 2013 Apr 2. 15, 117

[139] E. Rieter, F. Vinke, D. Bakula, E. Cebollero, C. Ungermann, T. Proikas-Cezanne, and F. Reggiori. Atg18 function in autophagy is regulated by specific sites within its beta-propeller. Journal of Cell Science, 126 (2):593-604, 2013. 117IA Times Cited:10 Cited References Count:51. 15, 16, 119, 120

[140] T. Kobayashi, K. Suzuki, and Y. Ohsumi. Autophagosome formation can be achieved in the absence of atg 18 by expressing engineered pas-targeted atg2. FEBS Lett, 586(16):2473-2478, 2012. 977WF Times Cited:5 Cited References Count:30. 15, 101

[141] D. Romanyuk, A. Polak, A. Maleszewska, M. Sienko, M. Grynberg, and T. Zoladek. Human hatg2a protein expressed in yeast is recruited to preautophagosomal structure but does not complement autophagy defects of atg2 delta strain. Acta Biochimica Polonica, 58(3):365-374, 2011. 828GA Times Cited:1 Cited References Count:40. 16, 101, 103, 104

[142] E. Cebollero, A. van der Vaart, M. Zhao, E. Rieter, D. J. Klionsky, J. B. Helms, and F. Reggiori. Phosphatidylinositol-3-phosphate clearance plays a key role in autophagosome completion. Curr Biol, 22(17):1545-53, 2012. Cebollero, Eduardo van der Vaart, Aniek Zhao, Mantong Rieter, Ester Klionsky, Daniel J Helms, J Bernd Reggiori, Fulvio GM53396/GM/NIGMS NIH HHS/ R01 GM053396/GM/NIGMS NIH HHS/ England Curr Biol. 2012 Sep 11;22(17):1545-53. doi: 10.1016/j.cub.2012.06.029. Epub 2012 Jul 5. 16, 117

[143] A. K. Velikkakath, T. Nishimura, E. Oita, N. Ishihara, and N. Mizushima. Mammalian atg2 proteins are essential for autophagosome formation and important for regulation of size and distribution of lipid droplets. Mol Biol Cell, 23(5):896-909, 2012. Velikkakath, Anoop Kumar G Nishimura, Taki Oita, Eiko Ishihara, Naotada Mizushima, Noboru Mol Biol Cell. 2012 Mar;23(5):896-909. doi: 10.1091/mbc.E1109-0785. Epub 2012 Jan 4. 16, 101

[144] S. G. Pfisterer, D. Bakula, T. Frickey, A. Cezanne, D. Brigger, M. P. Tschan, H. Robenek, and T. ProikasCezanne. Lipid droplet and early autophagosomal membrane targeting of atg2a and atg141 in human tumor cells. Journal of Lipid Research, 55(7):1267-1278, 2014. Pfisterer, Simon G Bakula, Daniela Frickey, Tancred Cezanne, Alice Brigger, Daniel Tschan, Mario P Robenek, Horst Proikas-Cezanne, Tassula J Lipid Res. 2014 Apr 28;55(7):1267-1278. 16, 101 
[145] A. Velayos-Baeza, A. Vettori, R. R. Copley, C. Dobson-Stone, and A. P. Monaco. Analysis of the human vps13 gene family. Genomics, 84(3):536-49, 2004. Velayos-Baeza, Antonio Vettori, Andrea Copley, Richard R Dobson-Stone, Carol Monaco, A P Genomics. 2004 Sep;84(3):536-49. 16

[146] Y. Wang, Y. Wu, and D. Tang. The autophagy gene, atg18a, plays a negative role in powdery mildew resistance and mildew-induced cell death in arabidopsis. Plant Signal Behav, 6(9):1408-10, 2011. Wang, Yiping Wu, Yingying Tang, Dingzhong Plant Signal Behav. 2011 Sep;6(9):1408-10. 16, 17

[147] Y. Xiong, A. L. Contento, P. Q. Nguyen, and D. C. Bassham. Degradation of oxidized proteins by autophagy during oxidative stress in arabidopsis. Plant Physiol, 143(1):291-299, 2007. 124EP Times Cited:117 Cited References Count:44. 17

[148] Y. M. Liu, Y. Xiong, and D. C. Bassham. Autophagy is required for tolerance of drought and salt stress in plants. Autophagy, 5(7):954-963, 2009. 531TH Times Cited:44 Cited References Count:71. 17

[149] Z. B. Lai, F. Wang, Z. Y. Zheng, B. F. Fan, and Z. X. Chen. A critical role of autophagy in plant resistance to necrotrophic fungal pathogens. Plant Journal, 66(6):953-968, 2011. 783SI Times Cited:48 Cited References Count:65. 17, 18

[150] P. J. Rushton, I. E. Somssich, P. Ringler, and Q. X. J. Shen. Wrky transcription factors. Trends in Plant Science, 15(5):247-258, 2010. 603GU Times Cited:267 Cited References Count:98. 17

[151] M. R. Duan, J. Nan, Y. H. Liang, P. Mao, L. Lu, L. F. Li, C. H. Wei, L. H. Lai, Y. Li, and X. D. Su. Dna binding mechanism revealed by high resolution crystal structure of arabidopsis thaliana wrky1 protein. Nucleic Acids Research, 35(4):1145-1154, 2007. 152HT Times Cited:40 Cited References Count:53. 18

[152] K. Yamasaki, T. Kigawa, M. Inoue, M. Tateno, T. Yamasaki, T. Yabuki, M. Aoki, I. Seki, T. Matsuda, Y. Tomo, N. Hayami, T. Terada, M. Shirouzu, A. Tanaka, M. Seki, K. Shinozaki, and S. Yokoyama. Solution structure of an arabidopsis wrky dna binding domain. Plant Cell, 17(3):944-956, 2005. 907AB Times Cited:66 Cited References Count:47. 18

[153] B. K. Drobak and B. Heras. Nuclear phosphoinositides could bring fyve alive. Trends in Plant Science, 7(3):132-138, 2002. 528VH Times Cited:23 Cited References Count:53. 18, 121

[154] F. H. Niesen, H. Berglund, and M. Vedadi. The use of differential scanning fluorimetry to detect ligand interactions that promote protein stability. Nature Protocols, 2(9):2212-2221, 2007. 262HM Times Cited:428 Cited References Count:16. 33, 65

[155] R. A. Busse, A. Scacioc, J. M. Hernandez, R. Krick, M. Stephan, A. Janshoff, M. Thumm, and K. Kuhnel. Qualitative and quantitative characterization of protein-phosphoinositide interactions with liposomebased methods. Autophagy, 9(5):770-7, 2013. Busse, Ricarda A Scacioc, Andreea Hernandez, Javier M Krick, Roswitha Stephan, Milena Janshoff, Andreas Thumm, Michael Kuhnel, Karin Autophagy. 2013 May;9(5):770-7. doi: 10.4161/auto.23978. Epub 2013 Feb 27. 34, 35, 77, 117

[156] H. Eibl and W. E. M. Lands. A new, sensitive determination of phosphate. Analytical Biochemistry, 30 (1):51-, 1969. D6603 Times Cited:551 Cited References Count:12. 35 
[157] W. Kabsch. Automatic processing of rotation diffraction data from crystals of initially unknown symmetry and cell constants. Journal of Applied Crystallography, 26:795-800, 1993. 6 M1199 Times Cited:2590 Cited References Count:13. 36, 87

[158] P. D. Adams, P. V. Afonine, G. Bunkoczi, V. B. Chen, I. W. Davis, N. Echols, J. J. Headd, L. W. Hung, G. J. Kapral, R. W. Grosse-Kunstleve, A. J. McCoy, N. W. Moriarty, R. Oeffner, R. J. Read, D. C. Richardson, J. S. Richardson, T. C. Terwilliger, and P. H. Zwart. Phenix: a comprehensive pythonbased system for macromolecular structure solution. Acta Crystallogr D Biol Crystallogr, 66(Pt 2): 213-21, 2010. Adams, Paul D Afonine, Pavel V Bunkoczi, Gabor Chen, Vincent B Davis, Ian W Echols, Nathaniel Headd, Jeffrey J Hung, Li-Wei Kapral, Gary J Grosse-Kunstleve, Ralf W McCoy, Airlie J Moriarty, Nigel W Oeffner, Robert Read, Randy J Richardson, David C Richardson, Jane S Terwilliger, Thomas C Zwart, Peter H 082961/Wellcome Trust/United Kingdom GM063210/GM/NIGMS NIH HHS/ P01 GM063210/GM/NIGMS NIH HHS/ P01 GM063210-010002/GM/NIGMS NIH HHS/ P01 GM063210-020002/GM/NIGMS NIH HHS/ P01 GM063210-060002/GM/NIGMS NIH HHS/ P01 GM063210-070002/GM/NIGMS NIH HHS/ P01 GM063210-080002/GM/NIGMS NIH HHS/ P01 GM063210-090002/GM/NIGMS NIH HHS/ Wellcome Trust/United Kingdom England Acta Crystallogr D Biol Crystallogr. 2010 Feb;66(Pt 2):213-21. doi: 10.1107/S0907444909052925. Epub 2010 Jan 22. 36,88

[159] P. Emsley and K. Cowtan. Coot: model-building tools for molecular graphics. Acta Crystallographica Section D-Biological Crystallography, 60:2126-2132, 2004. 12 Sp. Iss. 1 874OU Times Cited:11433 Cited References Count: 19. 36, 40, 88

[160] D. van der Spoel, E Lindahl, B. Hess, G. Groenhof, A.E. Mark, and H.J.C. Berendsen. Gromacs: Fast, flexible and free. J. Comp. Chem., 26:1701-1718, 2005. 38, 39, 42, 43

[161] B. Hess, C. Kutzner, D. van der Spoel, and E. Lindahl. Gromacs 4: Algorithms for highly efficient, load-balanced, and scalable molecular simulation. J. Chem. Theory Comp., 4(3):435-447, 2008. 38, 39, 42,43

[162] W. F. van Gunsteren, S. R. Billeter, A. A. Eising, P. H. Hünenberger, P. Krüger, A. E. Mark, W. R. P. Scott, and I. G Tironi. Biomolecular Simulation: The GROMOS96 Manual and User Guide. Biomos Hochschulverlag, an der ETH Zurich, Groningen, Germany, 1996. 39, 41

[163] J. Hermans, H. J. C. Berendsen, W. F. van Gunsteren, and J. P. M. Postma. A consistent empirical potential for water-protein interactions. Biopolymers, 23:1513-1518, 1984. 39, 43

[164] T. Darden, D. York, and L. Pedersen. Particle mesh ewald: An n.log(n) method for ewald sums in large systems. J. Chem. Phys., 98:10089-10092, 1993. 39,43

[165] U. Essmann, L. Perera, M. L. Berkowitz, T. Darden, H. Lee, and L. G. Pedersen. A smooth particle mesh ewald method. J. Chem. Phys., 103:8577-8593, 1995. 39, 43

[166] G. Bussi, D. Donadio, and M. Parrinello. Canonical sampling through velocity rescaling. J. Chem. Phys., 126(1):014101, 2007. 39 
[167] S. Nosé and M. L. Klein. Constant pressure molecular dynamics for molecular systems. Mol. Phys., 50: 1055-1076, 1983. 39, 43

[168] B. Hess, H. Bekker, H. J. C. Berendsen, and J. G. E. M. Fraaije. Lincs: A linear constraint solver for molecular simulations. J. Comput. Chem., 18:1463-1472, 1997. 40,43

[169] G. M. Morris, R. Huey, W. Lindstrom, M. F. Sanner, R. K. Belew, D. S. Goodsell, and A. J. Olson. AutoDock4 and AutoDockTools4: Automated docking with selective receptor flexibility. J. Comput. Chem., 30(16):2785-2791, 2009. 40, 46, 47, 48

[170] D. S. Goodsell, G. M. Morris, and A. J. Olson. Automated docking of flexible ligands: applications of AutoDock. J. Mol. Recognit., 9:1-5, 1996. 41

[171] A. C. Wallace, R. A. Laskowski, and J. M. Thornton. LIGPLOT: a program to generate schematic diagrams of protein-ligand interactions. Protein Eng., 8:127-134, 1996. 41

[172] D. M. van Aalten, R. Bywater, J. B. Findlay, M. Hendlich, R. W. Hooft, and G. Vriend. PRODRG, a program for generating molecular topologies and unique molecular descriptors from coordinates of small molecules. J. Comput.-Aided Mol. Des., 10:255-262, 1996. 41

[173] L. D. Schuler, P. Walde, P. L. Luisi, and W. F. van Gunsteren. Molecular dynamics simulation of ndodecyl phosphate aggregate structures. Eur Biophys J., 30(5):330-343, 2001. 41

[174] I. Chandrasekhar, M. Kastenholz, R .D. Lins, C. Oostenbrink, L. D. Schuler, D.P. Tieleman, and W. F. van Gunsteren. A consistent potential energy parameter set for lipids: dipalmitoylphosphatidylcholine as a benchmark of the GROMOS96 45A3 force field. Eur Biophys J., 32(1):67-77, 2003. 41

[175] S. J. Marrink, H. J. Risselada, S. Yefimov, D. P. Tieleman, and A. H. de Vries. The martini force field: Coarse grained model for biomolecular simulations. Journal of Physical Chemistry B, 111(27):78127824, 2007. 186EJ Times Cited:968 Cited References Count:87. 41,42

[176] H. J. C. Berendsen, J. P. M. Postma, W. F. Vangunsteren, A. Dinola, and J. R. Haak. Molecular-dynamics with coupling to an external bath. Journal of Chemical Physics, 81(8):3684-3690, 1984. Tq735 Times Cited:11853 Cited References Count:39. 42

[177] K. A. Scott, P. J. Bond, A. Ivetac, A. P. Chetwynd, S. Khalid, and M. S. P. Sansom. Coarse-grained md simulations of membrane protein-bilayer self-assembly. Structure, 16(4):621-630, 2008. 287QT Times Cited:90 Cited References Count:73. 42

[178] K. Balali-Mood, P. J. Bond, and M. S. Sansom. Interaction of monotopic membrane enzymes with a lipid bilayer: a coarse-grained md simulation study. Biochemistry, 48(10):2135-45, 2009. Balali-Mood, Kia Bond, Peter J Sansom, Mark S P Biotechnology and Biological Sciences Research Council/United Kingdom Biochemistry. 2009 Mar 17;48(10):2135-45. doi: 10.1021/bi8017398. 42, 54 
[180] C. Oostenbrink, A. Villa, A. E. Mark, and W. F. Van Gunsteren. A biomolecular force field based on the free enthalpy of hydration and solvation: The gromos force-field parameter sets 53a5 and 53a6. Journal of Computational Chemistry, 25(13):1656-1676, 2004. 847FW Times Cited:992 Cited References Count:92. 43

[181] O. Berger, O. Edholm, and F. Jahnig. Molecular dynamics simulations of a fluid bilayer of dipalmitoylphosphatidylcholine at full hydration, constant pressure, and constant temperature. Biophysical Journal, 72(5):2002-2013, 1997. Wv984 Times Cited:926 Cited References Count:42. 43

[182] D. J. Evans and B. L. Holian. The nose-hoover thermostat. Journal of Chemical Physics, 83(8):40694074, 1985. Asa86 Times Cited:325 Cited References Count:17. 43

[183] W. Humphrey, A. Dalke, and K. Schulten. VMD: Visual molecular dynamics. J. Mol. Graphics, 14: 33-38, 1996. 43

[184] W. L. DeLano. The PyMOL molecular graphics system, version 1.3. Schrödinger, LLC, 2002. 43, 44, 92

[185] P. Tommaso, S. Moretti, I. Xenarios, M. Orobitg, A. Montanyola, J. M. Chang, J. F. Taly, and C. Notredame. T-coffee: a web server for the multiple sequence alignment of protein and rna sequences using structural information and homology extension. Nucleic Acids Res, 39:W13-W17, 2011. Suppl. 2 786RJ Times Cited:4 Cited References Count:25. 43, 52, 97

[186] N. Eswar, B. Webb, M. A. Marti-Renom, M. S. Madhusudhan, D. Eramian, M. Y. Shen, U. Pieper, and A. Sali. Comparative protein structure modeling using modeller. Curr Protoc Bioinformatics, Chapter 5:Unit 5 6, 2006. Eswar, Narayanan Webb, Ben Marti-Renom, Marc A Madhusudhan, M S Eramian, David Shen, Min-Yi Pieper, Ursula Sali, Andrej P01 A135707/PHS HHS/ P01 GM071790/GM/NIGMS NIH HHS/ P01 GM71790/GM/NIGMS NIH HHS/ R01 GM054762/GM/NIGMS NIH HHS/ R01 GM54762/GM/NIGMS NIH HHS/ U54 GM62529/GM/NIGMS NIH HHS/ Curr Protoc Bioinformatics. 2006 Oct;Chapter 5:Unit 5.6. doi: 10.1002/0471250953.bi0506s15. 43

[187] M. A. Marti-Renom, A. C. Stuart, A. Fiser, R. Sanchez, F. Melo, and A. Sali. Comparative protein structure modeling of genes and genomes. Annu Rev Biophys Biomol Struct, 29:291-325, 2000. MartiRenom, M A Stuart, A C Fiser, A Sanchez, R Melo, F Sali, A GM 54762/GM/NIGMS NIH HHS/ Annu Rev Biophys Biomol Struct. 2000;29:291-325. 43

[188] R. A. Laskowski, M. W. Macarthur, D. S. Moss, and J. M. Thornton. Procheck - a program to check the stereochemical quality of protein structures. Journal of Applied Crystallography, 26:283-291, 1993. 2 Ky851 Times Cited:14497 Cited References Count:22. 43

[189] L. Monticelli, S. K. Kandasamy, X. Periole, R. G. Larson, D. P. Tieleman, and S. J. Marrink. The martini coarse-grained force field: Extension to proteins. Journal of Chemical Theory and Computation, 4(5): 819-834, 2008. 300CU Times Cited:554 Cited References Count:78. 55

[190] X. Periole, M. Cavalli, S. J. Marrink, and M. A. Ceruso. Combining an elastic network with a coarsegrained molecular force field: Structure, dynamics, and intermolecular recognition. Journal of Chemical 
Theory and Computation, 5(9):2531-2543, 2009. 490GP Times Cited:77 Cited References Count:102. 55

[191] S. O. Yesylevskyy, L. V. Schafer, D. Sengupta, and S. J. Marrink. Polarizable water model for the coarse-grained martini force field. PLoS Comput Biol, 6(6):e1000810, 2010. Yesylevskyy, Semen O Schafer, Lars V Sengupta, Durba Marrink, Siewert J PLoS Comput Biol. 2010 Jun 10;6(6):e1000810. doi: 10.1371/journal.pcbi.1000810. 57

[192] R. A. Busse. Insights into membrane binding of PROPPINs and Reconstitution of mammalian autophagic conjugation systems. PhD thesis, 2013. 61, 67, 69, 77, 83, 113, 114, 116, 117, 118, 119, 123

[193] F. W. Studier. Protein production by auto-induction in high density shaking cultures. Protein Expr Purif, 41(1):207-34, 2005. Studier, F William P50 GM062529-05/GM/NIGMS NIH HHS/ Protein Expr Purif. 2005 May;41(1):207-34. 61, 67

[194] D. M. Francis and R. Page. Strategies to optimize protein expression in e. coli. Curr Protoc Protein Sci, Chapter 5:Unit 524 1-29, 2010. Francis, Dana M Page, Rebecca Curr Protoc Protein Sci. 2010 Aug;Chapter 5:Unit 5.24.1-29. doi: 10.1002/0471140864.ps0524s61. 62, 75, 107

[195] S. Amlacher, P. Sarges, D. Flemming, V. van Noort, R. Kunze, D. P. Devos, M. Arumugam, P. Bork, and E. Hurt. Insight into structure and assembly of the nuclear pore complex by utilizing the genome of a eukaryotic thermophile. Cell, 146(2):277-289, 2011. 795XS Times Cited:50 Cited References Count:46. 72, 104

[196] M. R. Dyson, S. P. Shadbolt, K. J. Vincent, R. L. Perera, and J. McCafferty. Production of soluble mammalian proteins in escherichia coli: identification of protein features that correlate with successful expression. Bmc Biotechnology, 4, 2004. 890QF Times Cited:111 Cited References Count:58. 75, 107

[197] C. Bieniossek, Y. Nie, D. Frey, N. Olieric, C. Schaffitzel, I. Collinson, C. Romier, P. Berger, T. J. Richmond, M. O. Steinmetz, and I. Berger. Automated unrestricted multigene recombineering for multiprotein complex production. Nat Methods, 6(6):447-50, 2009. Bieniossek, Christoph Nie, Yan Frey, Daniel Olieric, Natacha Schaffitzel, Christiane Collinson, Ian Romier, Christophe Berger, Philipp Richmond, Timothy J Steinmetz, Michel O Berger, Imre Nat Methods. 2009 Jun;6(6):447-50. doi: 10.1038/nmeth.1326. Epub 2009 May 3. 75, 108

[198] A. D'Arcy, F. Villard, and M. Marsh. An automated microseed matrix-screening method for protein crystallization. Acta Crystallogr D Biol Crystallogr, 63(Pt 4):550-4, 2007. D’Arcy, Allan Villard, Frederic Marsh, May Denmark Acta Crystallogr D Biol Crystallogr. 2007 Apr;63(Pt 4):550-4. Epub 2007 Mar 16. 83

[199] A. Dong, X. Xu, A. M. Edwards, C. Chang, M. Chruszcz, M. Cuff, M. Cymborowski, R. Di Leo, O. Egorova, E. Evdokimova, E. Filippova, J. Gu, J. Guthrie, A. Ignatchenko, A. Joachimiak, N. Klostermann, Y. Kim, Y. Korniyenko, W. Minor, Q. Que, A. Savchenko, T. Skarina, K. Tan, A. Yakunin, A. Yee, V. Yim, R. Zhang, H. Zheng, M. Akutsu, C. Arrowsmith, G. V. Avvakumov, A. Bochkarev, L. G. 
Dahlgren, S. Dhe-Paganon, S. Dimov, L. Dombrovski, Jr. Finerty, P., S. Flodin, A. Flores, S. Graslund, M. Hammerstrom, M. D. Herman, B. S. Hong, R. Hui, I. Johansson, Y. Liu, M. Nilsson, L. Nedyalkova, P. Nordlund, T. Nyman, J. Min, H. Ouyang, H. W. Park, C. Qi, W. Rabeh, L. Shen, Y. Shen, D. Sukumard, W. Tempel, Y. Tong, L. Tresagues, M. Vedadi, J. R. Walker, J. Weigelt, M. Welin, H. Wu, T. Xiao, H. Zeng, and H. Zhu. In situ proteolysis for protein crystallization and structure determination. Nat Methods, 4(12):1019-21, 2007. Dong, Aiping Xu, Xiaohui Edwards, Aled M Midwest Center for Structural Genomics Structural Genomics Consortium Chang, Changsoo Chruszcz, Maksymilian Cuff, Marianne Cymborowski, Marcin Di Leo, Rosa Egorova, Olga Evdokimova, Elena Filippova, Ekaterina Gu, Jun Guthrie, Jennifer Ignatchenko, Alexandr Joachimiak, Andrzej Klostermann, Natalie Kim, Youngchang Korniyenko, Yuri Minor, Wladek Que, Qiuni Savchenko, Alexei Skarina, Tatiana Tan, Kemin Yakunin, Alexander Yee, Adelinda Yim, Veronica Zhang, Rongguang Zheng, Hong Akutsu, Masato Arrowsmith, Cheryl Avvakumov, George V Bochkarev, Alexey Dahlgren, Lars-Goran Dhe-Paganon, Sirano Dimov, Slav Dombrovski, Ludmila Finerty, Patrick Jr Flodin, Susanne Flores, Alex Graslund, Susanne Hammerstrom, Martin Herman, Maria Dolores Hong, Bum-Soo Hui, Raymond Johansson, Ida Liu, Yongson Nilsson, Martina Nedyalkova, Lyudmila Nordlund, Par Nyman, Tomas Min, Jinrong Ouyang, Hui Park, Hee-won Qi, Chao Rabeh, Wael Shen, Limin Shen, Yang Sukumard, Deepthi Tempel, Wolfram Tong, Yufeng Tresagues, Lionel Vedadi, Masoud Walker, John R Weigelt, Johan Welin, Martin Wu, Hong Xiao, Ting Zeng, Hong Zhu, Haizhong GM074942/GM/NIGMS NIH HHS/ U54 GM074942/GM/NIGMS NIH HHS/ U54 GM074942-02/GM/NIGMS NIH HHS/ Wellcome Trust/United Kingdom Nat Methods. 2007 Dec;4(12):1019-21. Epub 2007 Nov 4. 83

[200] G. Evans and R. F. Pettifer. Chooch: a program for deriving anomalous-scattering factors from x-ray fluorescence spectra. Journal of Applied Crystallography, 34:82-86, 2001. 1 394PQ Times Cited:160 Cited References Count:28. 87

[201] G. M. Sheldrick. Experimental phasing with shelxc/d/e: combining chain tracing with density modification. Acta Crystallographica Section D-Biological Crystallography, 66:479-485, 2010. 4 573US Times Cited:228 Cited References Count:39. 88

[202] A. B. Seven, K. D. Brewer, L. Shi, Q. X. Jiang, and J. Rizo. Prevalent mechanism of membrane bridging by synaptotagmin-1. Proceedings of the National Academy of Sciences of the United States of America, 110(34):E3243-E3252, 2013. 203DU Times Cited:5 Cited References Count:50. 96

[203] C. Cole, J. D. Barber, and G. J. Barton. The jpred 3 secondary structure prediction server. Nucleic Acids Res, 36(Web Server issue):W197-201, 2008. Cole, Christian Barber, Jonathan D Barton, Geoffrey J England Nucleic Acids Res. 2008 Jul 1;36(Web Server issue):W197-201. doi: 10.1093/nar/gkn238. Epub 2008 May 7. 103, 104, 107

[204] Z. Li, R. M. Venable, L. A. Rogers, D. Murray, and R. W. Pastor. Molecular dynamics simulations of pip2 and pip3 in lipid bilayers: Determination of ring orientation, and the effects of surface roughness on a poisson- boltzmann description. Biophysical Journal, 97(1):155-163, 2009. 469BV Times Cited:24 Cited References Count:52. 113 
[205] E. Psachoulia and M.S.P. Sansom. Interactions of the pleckstrin homology domain with phosphatidylinositol phosphate and membranes: characterization via molecular dynamics simulations. Biochem., 47: 4211-4220, 2008. 113

[206] E. Psachoulia and M. S. P. Sansom. Px- and fyve-mediated interactions with membranes: Simulation studies. Biochemistry, 48(23):5090-5095, 2009. 456PX Times Cited:10 Cited References Count:40. 113

[207] T. R. Jeffries, S. K. Dove, R. H. Michell, and P. J. Parker. Ptdins-specific mpr pathway association of a novel wd40 repeat protein, wipi49. Molecular Biology of the Cell, 15(6):2652-2663, 2004. 825SJ Times Cited:65 Cited References Count:37. 121 


\section{Andreea Scacioc}

MSc. Molecular and Cellular Biology

$\begin{array}{lll}\text { DATE OF BIRTH: } & \text { December 14, } 1985 \text { (Constanta, Romania) } \\ \text { NATIONALITY: } & \text { Romanian } \\ \text { CITIZENSHIP: } & \text { Romanian } \\ \text { MARITAL STATUS: } & \text { Single } & \\ \text { CONTACT INFO: } & \text { Address: } & \text { Ulrideshuser strasse 14 } \\ & & 37077 \text { Göttingen, Germany } \\ & \text { Phone: } & +49(0) 1737346534 \\ & \text { E-mail: } & \text { ascacio@gwdg.de }\end{array}$

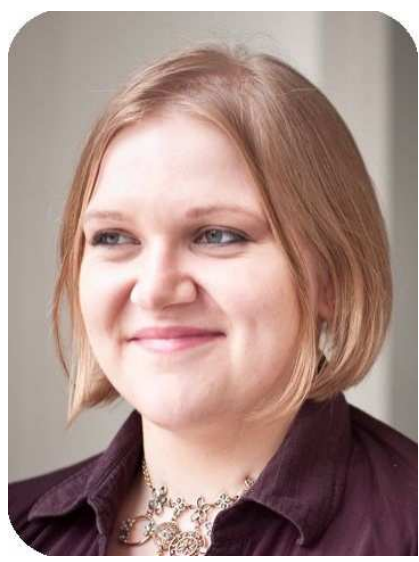

PhD studies

Program: Biomolecules: Structure - Function Dynamics

Master of Science

Major: Biological Recognition

Specialization: Molecular and Cellular Biology

Bachelor of Science

Major: Physics following a pre-med track

High school diploma

Specialization: Mathematical Studies and Computer International Computer High School, Constanta, Science

\section{SCIENTIFIC EXPERIENCE}

Molecular Mechanism of Autophagy group of Dr. Karin Kühnel

May 2011 - present

Neurobiology department of Prof. Reinhard Jahn

Max Planck Institute for Biophysical Chemistry, Göttingen, Germany

$P h D$ student structurally characterizing autophagy proteins and their binding partners.

\section{Computational Chemistry group of Prof. Danilo Roccatano}

Oct 2009 - Nov 2010 Jacobs University Bremen, Germany

PhD student in a project involving rational design and molecular dynamics study of T4 RNA ligase 1.

\section{SeSaM-Biotech GmbH}

Oct 2009 - Oct 2010

Bremen, Germany

PhD student developing SeSaM Step 1 (SeSaM-Fragmenter, a computational tool to predict the outcome of this step) and Step 2 (directed evolution and rational design of T4 RNA ligase 1 for molecular biology applications). Also involved in data analysis for commercial projects and internal R\&D.

Jacobs University Bremen, Germany 
Master thesis student studying the ssDNA fragment size distributions in the first step of SeSaM in order to develop a computational tool to predict the outcome of SeSaM Step 1.

\section{Cell Biology group of Prof. Klaudia Brix}

May - Aug 2008

Jacobs University Bremen, Germany

Master Student studying the role of Cathepsins in Thyroid cancer cell lines.

\section{Immunology group of Prof. Sebastian Springer}

Feb - May 2008

Jacobs University Bremen, Germany

Master Student developing a negative control for the COPII budding project.

\section{Protein Engineering group of Prof. Ulrich Schwaneberg}

Sep 2007 - Feb 2008

Jacobs University Bremen, Germany

Master Student developing a new method for screening glucose oxidase libraries in yeast using flow cytometry.

\section{Dr. Olivier Cuvillier and Prof. Bernard Malavaud group}

Jun- Aug 2006 \& Jul - Sep 2007

IPBS (Institute de Pharmacologie et Biologie Structurle), Toulouse, France

Intern with a project investigating the modifications in PC3 and NIH3T3 cells after cryotherapy.

Modifications studied involved the apoptosis pathways involving ceramide and sphingosine kinase

\section{Biophysics group of Prof. Mathias Winterhalter}

Jun 2005 - Jul 2007

International University of Bremen, Germany

Student Assistant in the projects: Control of Enzymatic Activity by Encapsulation into Magnetic Liposomes Project and Characterization of Magnetic Liposomes.

\section{EXPERIMENTAL SKILLS}

molecular cloning, protein expression in E. Coli and S. cerevisiae, protein purification, protein crystallography, isothermal titration calorimetry, circular dichroism, liposomes preparation, lipid content determination, flotation assays, fluorescence based assays, dynamic light scattering, enzymatic activity determination with chemiluminescence and absorbance, enzyme kinetics and inhibition assays, statistical analysis of polymerase fidelity, acrylamide gels (native and urea) for DNA analysis, Real Time PCR, mammalian cell culture, flow cytometry, preparation of alginate beads and water in oil emulsions for high throughput screening of enzymes presented on the yeast cell surface, western blotting, co-immunoprecipitation, COPII vesicles preparation, Confocal Laser Scanning Microscopy

\section{PUBLICATIONS}

Busse RA, Scacioc A, Hernandez JM, Krick R, Stephan M, Janshoff A, Thumm M, Kühnel K (2013) Qualitative and quantitative characterization of protein-phosphoinositide interactions with liposomebased methods. Autophagy 9(5):770-7

Thumm M, Busse RA, Scacioc A, Stephan M, Janshoff A, Kühnel K, Krick R (2013) It takes two to tango: PROPPINs use two phosphoinositide-binding sites. Autophagy 9(1):106-7

Krick R, Busse RA, Scacioc A, Stephan M, Janshoff A, Thumm M, Kühnel K (2012) Structural and functional characterization of the two phosphoinositide binding sites of PROPPINs, a $\beta$-propeller protein family. Proc Natl Acad Sci U S A. 109(30):E2042-9

Mundhada H, Marienhagen J, Scacioc A, Schenk A, Roccatano D, Schwaneberg U (2011) SeSaMTv-II generates a protein sequence space which is unobtainable by epPCR. ChemBioChem.

12(10):1595-601 
Prodanovic R, Ostafe R, Scacioc A, Schwaneberg U (2011) Ultrahigh-Throughput Screening System for Directed Glucose Oxidase Evolution in Yeast Cells. Comb Chem High Throughput Screen.

14(1):55-60

\section{CONFERENCES, SUMMER SCHOOLS \& WORKSHOPS}

15th International Conference on the Crystallization of

17-20 September 2014 Biological Macromolecules

Hamburg, Germany

Presenter of the poster: Structural characterization of PROPPINs

Keystone Symposia: Autophagy, Inflammation and Immunity (B4)

17-22 February 2014

Monreal, Canada

Presenter of the poster: Characterization of PROPPIN membrane binding

$9^{\text {th }}$ Horizons in Molecular Biology

8 - 11 October 2012

Göttingen, Germany

Presenter of the poster: Computational characterization of PROPPINs

$8^{\text {th }}$ Horizons in Molecular Biology

$14-17$ Sep 2011

Göttingen, Germany

Attendee

International Congress on Biocatalysis -

$5-7$ Oct 2010

biocat2010

Hamburg, Germany

Presenter of the poster: Removal of Mutagenic Hotspots by Uniform Distribution of Mutations in SeSaM

IBN-Forum Innovative Ansätze für die Bioraffinerie der Zukunft

29 Aug - 2 Sep 2010

Hamburg, Germany

Attendee

Deutsche Biotechnologie Tage

21 - 22 Apr 2010

Berlin, Germany

Attendee

Biosensing with channels Summer School

31 Jul - 5 Aug 2005

International University of Bremen, Germany

Presenter of the poster: Control of Enzymatic Activity by Encapsulation into Magnetic Liposomes 


\section{Neurobiology Department}

May 2012 - Dec 2013

Max Planck Institute for Biophysical Chemistry, Göttingen, Germany

Method course supervisor for method courses: Protein purification and characterization;

Crystallization and X-Ray structure determination of biological macromolecules; Bioanalytics

Project Supervisor for master students: Sebastian David and Oleksandr Yagensky

\section{SeSaM-Biotech GmbH}

Feb - May 2010

Bremen, Germany

Project Supervisor for bachelor students: Anita Smarandache and Alexandra Pinzaru

\section{Prof. Danilo Roccatano}

Mar - Apr 2010

Jacobs University Bremen, Germany

Lecturer for Design of Biological Molecules and Systems II and MoLife Seminar; responsible for holding one lecture for each class in lieu of Prof. Danilo Roccatano

\section{Physics Department}

Sep 2005 - May 2009

Jacobs University Bremen, Germany

Tutor for Physics Support Center

Teaching Assistant for NatSci Physics Lab II; Advanced Physics BI (General relativity,

electrodynamics and optics) and for Advanced Physics All (Quantum Mechanics)

\section{Mathematics Department}

Sep 2005 - May 2007

Jacobs University Bremen, Germany

Tutor for Math Support Center

\section{AWARDS AND CERTIFICATES}

Proficiency Cambridge Certificate for English as a Foreign Language (grade B)

Dec 2004

Advanced Cambridge Certificate for English as a Foreign Language (grade A) Jun 2004

Multiple awards in national (Romania) contests and Olympiads of physics and two qualifications (2003 \& 2004) in the National Team for the International Physics Olympiad

\section{COMPUTER SKILLS}

Programming languages: $\quad \mathrm{C}, \mathrm{C}++$, Pascal, Delphi, Mathematica, Matlab, Fortran, Standard ML Molecular modeling: $\quad$ VMD, PyMOL, Modeller, GROMACS, AutoDock

Crystallography software: Phenix, Coot, XDS, CCP4

Software:

Operating systems: LAteX, Origin, Adobe Photoshop, Adobe Illustrator, Adobe InDesign Windows, Ubuntu

\section{LANGUAGES}

$\begin{array}{lll}\text { English: } & \text { Fluent } & \text { Romanian: native }\end{array}$




\section{Max Planck Society}

Oct 2012 - present

Elected member of the steering group of PhDnet and Spokesperson for PhDnet for 2014. PhDnet is a network of all Max Planck PhD candidates represented by the steering group.

\section{Göttingen Graduate School for Neurosciences,}

Jun 2012 - Sep 2014

Biophysics, and Molecular Biosciences

Göttingen, Germany

Elected program representative for Biomolecules: Structure - Function - Dynamics; Biomolecules 2012 \& 2013 retreat organizer; Summer Games 2012 organizer.

\section{Dorothea Schlözer Mentoring Program}

Georg-August Universität Göttingen , Germany

Mentee.

\section{Jacobs University Bremen}

Oct 2005 - Jun 2009

Bremen, Germany

PR Task Force of the Undergraduate Student Government; Volunteer in IUB Kids; founding member of Anti-Discrimination Team; member of Debating Society; organizer of Glamour Day 2008; organizer Apartment 0-815 Awards 2008; organizer Romanian booth in the International Fair 2009

\section{REFERENCES}

\section{Dr. Karin Kühnel}

Group leader - Autophagy

Max Planck Institute for Biophysical Chemistry

Am Fassberg 11, 37077 Göttingen, Germany

E-mail: kkuehne@gwdg.de

Telephone: ++49 551 201-1795

\section{Prof. Dr. Reinhard Jahn}

Max Planck Director - Neurobiology

Max Planck Institute for Biophysical Chemistry

Am Fassberg 11, 37077 Göttingen, Germany

E-mail: rjahn@gwdg.de 